Annette Olbrisch

\title{
Die Zinsmarge und ihre Determinanten
}




\section{Annette Olbrisch}

\section{Die Zinsmarge und ihre Determinanten}

Die Analyse des internationalen Handels von Dienstleistungen weist Eigenarten auf, die dem Charakter der Dienstleistung geschuldet sind: Immateriell und nicht lagerfähig findet dennoch der internationale Austausch statt, wenn auch unter besonderen Bedingungen. Diese Arbeit untersucht den Handel von Bankdienstleistungen und die Rahmenbedingungen denen er unterworfen ist. Die Anforderungen an die Dienstleister und ihre Produkte sind länderspezifisch sehr unterschiedlich gestaltet. Als Indikator für protektionistische Beschränkungen wird die landeseigene Zinsmarge verwendet, die die Wettbewerbssituation reflektiert. Durch entsprechende Regressionsschätzungen werden die Protektionspotentiale und -mechanismen eines Landes ermittelt sowie die wesentlichen Protektionsfaktoren identifiziert. Im Rahmen internationaler Verhandlungen sollten diese Handelshemmnisse vorrangig reduziert werden, um möglichst große Wohlfahrtsgewinne für die beteiligten Länder zu generieren.

Annette Olbrisch studierte Volkswirtschaftslehre an der Universität Hamburg sowie der Sorbonne in Paris und arbeitete als Wissenschaftliche Mitarbeiterin an der RWTH Aachen sowie der Helmut-Schmidt-Universität/Universität der Bundeswehr Hamburg. 
Die Zinsmarge und ihre Determinanten 


\title{
SCHRIFTEN ZUR \\ WIRTSCHAFTSTHEORIE UND WIRTSCHAFTSPOLITIK
}

\author{
Herausgegeben von \\ Klaus Beckmann, Michael Berlemann, Rolf Hasse, \\ Jörn Kruse, Franco Reither, Wolf Schäfer, \\ Thomas Straubhaar und Klaus W. Zimmermann
}

\section{Band 38}

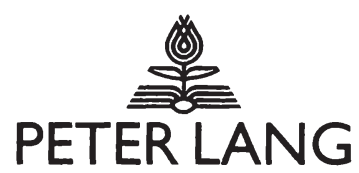

Frankfurt am Main - Berlin - Bern - Bruxelles - New York - Oxford - Wien 


\section{Annette Olbrisch}

\section{Die Zinsmarge und ihre Determinanten}

Eine theoretische und empirische Analyse unter besonderer Berücksichtigung von Protektion im Bankensektor

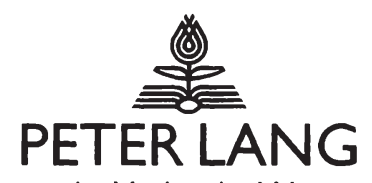

InternationalerVerlag derWissenschaften

Annette D. Olbrisch - 978-3-631-75128-2 


\section{Bibliografische Information der Deutschen Nationalbibliothek}

Die Deutsche Nationalbibliothek verzeichnet diese Publikation in der Deutschen Nationalbibliografie; detaillierte bibliografische Daten sind im Internet über http://dnb.d-nb.de abrufbar.

Open Access: The online version of this publication is published on www.peterlang.com and www.econstor.eu under the international Creative Commons License CC-BY 4.0. Learn more on how you can use and share this work: http://creativecommons.org/licenses/ by/4.0.

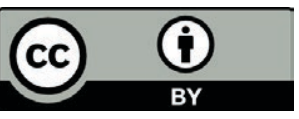

This book is available Open Access thanks to the kind support of ZBW - Leibniz-Informationszentrum Wirtschaft.

Zugl.: Hamburg, Univ. der Bundeswehr, Diss., 2008

Gedruckt auf alterungsbeständigem, säurefreiem Papier.

D 705

ISSN 1433-1519

ISBN 978-3-631-58104-9

ISBN 978-3-631-75128-2 (eBook)

(C) Peter Lang $\mathrm{GmbH}$ Internationaler Verlag der Wissenschaften

Frankfurt am Main 2009

Alle Rechte vorbehalten.

Das Werk einschließlich aller seiner Teile ist urheberrechtlich geschützt. Jede Verwertung außerhalb der engen Grenzen des

Urheberrechtsgesetzes ist ohne Zustimmung des Verlages unzulässig und strafbar. Das gilt insbesondere für Vervielfältigungen, Übersetzungen, Mikroverfilmungen und die Einspeicherung und Verarbeitung in elektronischen Systemen. 


\section{Meinen Eltern}

Brigitte und Richard Olbrisch 
Annette D. Olbrisch - 978-3-631-75128-2 Downloaded from PubFactory at 01/11/2019 07:59:04AM via free access 


\section{Vorwort}

Das Verfassen einer Dissertation stellt einen langwierigen und zuweilen auch durchaus turbulenten Prozeß dar. Auf diesem habe mich zahlreiche Personen mit ihrem Rat, ihren Meinungen und ihrer Hilfe begleitet. Mit großem Engagement und viel Energie hat Prof. Dr. Wolf Schäfer diese Arbeit begleitet, wofür ich ihm herzlich danke. Auch Prof. Klaus W. Zimmermann gebührt mein Dank für die tatkräftige Unterstützung während der finalen Phase. Für die erfahrene Unterstützung danke ich auch Herrn Prof. Dr. Dr. W. Gocht ( $\dagger$ ), sowie den Kollegen und Freunden an der RWTH Aachen wie auch an der Helmut-Schmidt-Universität in Hamburg, insbesondere Annie Seubert, Dorothee Söndgen, Dr. Monique Radisch, Susan Kurth, Dr. Christoph Kimmel und Dr. Sven Schulze. Für seine Unterstützung und sein Interesse an meiner Arbeit bin ich Herrn Prof. Franco Reither tief verbunden.

Den größten emotionalen Rückhalt während meiner Promotionszeit erhielt ich durch meine Familie. Eine große Stütze war und ist mein Bruder David - mit seinem gelassenen Blick auf das Leben rückt er die Relationen immer wieder zurecht. Ohne die liebevolle Unterstützung meiner Eltern wäre dieses Buch nicht entstanden, weshalb ich es ihnen widme.

Annette Olbrisch

Hamburg, im November 2007 
Annette D. Olbrisch - 978-3-631-75128-2 Downloaded from PubFactory at 01/11/2019 07:59:04AM via free access 


\section{Inhaltsverzeichnis}

Tabellenverzeichnis 11

Abbildungsverzeichnis $\quad 13$

$\begin{array}{ll}\text { Verzeichnis der Modell-Variablen } & 14\end{array}$

1 Einleitung 15

2 Internationaler Dienstleistungshandel $\quad 18$

$\begin{array}{ll}2.1 \text { Protektionismus und GATS } & 18\end{array}$

2.1.1 Protektion im Welthandel 18

2.1.2 Das GATS als Instrument zur Protektionsreduzierung 20

2.1.2.1 Grundlegende Prinzipien des GATS 25

2.1.2.2 Charakteristika und Defizite des GATS 29

2.2 Internationaler (Bank-)Dienstleistungshandel 31

2.2.1 Banken als Dienstleistungsunternehmen 31

2.2.1.1 Die bank- bzw. einzelwirtschaftliche Sichtweise 33

2.2.1.2 Die volkswirtschaftliche Funktion von Banken $\quad 35$

2.2.2 Dienstleistungsverkehr $\quad 37$

2.2.2.1 Formen des Handels $\quad 37$

2.2.2.2 Wichtige Aspekte internationaler

Dienstleistungstransaktionen $\quad 39$

2.3.2 Hemmnisse im Dienstleistungshandel 43

$\begin{array}{ll}2.3 \text { Zwischenfazit } & 49\end{array}$

3 Zinsmargen als Indikator des Protektionsgrades $\quad 50$

3.1 Das Konzept der Zinsmarge $\quad 50$

3.2 Das erweiterte Dealer-Modell $\quad 57$

3.2.1 Die Annahmen des Modells $\quad 58$

3.2.2 Variation der administrativen Kosten $\quad 62$

3.2.3 Implikationen des Modells 64

3.3 Zinsmargen: Aktuelle Daten und ihre Entwicklung 65

3.3.1 Gesamtentwicklung der Zinsmargen 65

3.3.2 Ländergruppe I (Einkommensklasse > 15.000 US-Dollar) 71

3.3.3 Ländergruppe II (Einkommensklasse 5.000 - 14.999 US-Dollar) 73

3.3.4 Ländergruppe III (Einkommensklasse 1.000 - 4.999 US-Dollar) 75

3.3.5 Ländergruppe IV (Einkommensklasse < 1.000 US-Dollar) 77

4 Determinanten der Zinsmargen - eine empirische Analyse 81

$\begin{array}{ll}\text { 4.1 Daten und Methoden } & 81\end{array}$

4.1.1 Die Datenbasis $\quad 81$ 
4.1.1.1 Quellen $\quad 81$

4.1.1.2 Auswahl der Variablen: Kriterien $\quad 83$

4.1.2 Die Methodologie $\quad 83$

4.1.3 Variablengruppen und ihre Protektionspotentiale $\quad 87$

4.2 Regressionen der Variablengruppen $\quad 96$

4.2.1 Darstellung der Teilschätzungen 96

4.2.1.1 Kapitalquoten $\quad 96$

4.2.1.2 Attraktivität des Bankenmarktes (Gewinnpotential) 99

4.2.1.3 Eigenkapital der Banken 102

4.2.1.4 Verflechtungsgrad zwischen Banken und Finanzunternehmen 104

$\begin{array}{ll}\text { 4.2.1.5 Konzentrationsgrad } & 107\end{array}$

4.2.1.6 Geschäftsfelder der Banken $\quad 109$

4.2.1.7 Rechnungslegung $\quad 114$

4.2.1.8 Liquidität (im weiteren Sinne) 116

4.2.1.9 Einlagensicherungssysteme 119

4.2.1.10 Öffentliches Monitoring / Offenlegungspflichten $\quad 122$

4.2.1.11 Haftbarkeit bzw. juristische Belangbarkeit des Bankmanagements 124

4.2.1.12 Kontrollintensität $\quad 127$

4.2.1.13 Economic Freedom Indicator 131

4.2.1.14 Nicht oder kaum signifikante Aspekte $\quad 132$

4.2.2 Erkenntnisse aus den Teilschätzungen 140

4.2.2.1 Der erste Untersuchungszeitraum 141

4.2.2.2 Der zweite Untersuchungszeitraum 144

4.3 Die aussagekrättigsten Schätzgleichungen 147

4.3.1 Aussagekräftigste Schätzgleichung 1998 - 2000 147

4.3.2 Aussagekräftigste Schätzgleichung 2001-2003 156

4.3.3 Erkenntnisse aus den Gesamtschätzungen 162

5 Fazit 169

Anhang A1: Legende der Variablen 173

Anhang A2: Länder der Untersuchung $\quad 181$

Anhang A3: Zinsmargen und Pro-Kopf-Einkommen 184

Anhang A4: Schätzergebnisse - Economic Freedom Indicator, Teil $2 \quad 187$

Anhang A5: Fragebogen der Weltbank-Umfrage, Periode 1998 - 2000 188

Anhang A6: Fragebogen der Weltbank-Umfrage, Periode 2001 - $2003 \quad 195$

Literaturverzeichnis 205 


\section{Tabellenverzeichnis}

Tab. 2-1: Handelshemmnisse im Dienstleistungsbereich 46

Tab. 3-1: Bewertungsschema des Trade Restrictiveness Index 55

Tab. 3-2: Statistik der Nettozinsmargen (für Länder in beiden Untersuchungsperioden) 65

Tab. 3-3: Statistik der Nettozinsmargen (ohne Ghana) 66

Tab. 3-4: Statistik der Nettozinsmargen (alle Länder) 69

Tab. 3-5: Ergebnistableau (> 15.000 US-Dollar) 71

Tab. 3-6: Ergebnistableau (5.000 - 14.999 US-Dollar) 73

Tab. 3-7: Ergebnistableau (1.000 - 4.999 US-Dollar) 75

Tab. 3-8: Ergebnistableau (<1.000 US-Dollar) 77

Tab. 3-9: Ergebnisübersicht für alle Einkommensklassen $\quad 80$

Tab. 4-1: Übersicht Protektionspotentiale 95

Tab. 4-2: Schätzergebnisse - Kapitalquoten $\quad 97$

Tab. 4-3: Signifikanz der Variablen „Kapital“" 98

Tab. 4-4: Schätzergebnisse - Attraktivität 100

Tab. 4-5: Signifikanz der Variablen „Attraktivität“" 101

Tab. 4-6: Schätzergebnisse - Eigenkapital 103

Tab. 4-7: Signifikanz der Variablen „Eigenkapital“ 103

Tab. 4-8: Schätzergebnisse - Verflechtung: Banken und Finanzunternehmen 105

Tab. 4-9: Signifikanz der Variablen „Verflechtung“ 106

Tab. 4-10: Schätzergebnisse - Konzentrationsgrad 108

Tab. 4-11: Signifikanz der Variablen „Konzentrationsgrad“ 108

Tab. 4-12: Schätzergebnisse - Geschäftsfelder der Banken 111

Tab. 4-13: Signifikanz der Variablen „Geschäftsfelder der Banken“ 113

Tab. 4-14: Schätzergebnisse - Rechnungslegung 115

Tab. 4-15: Signifikanz der Variablen „Rechnungslegung“ 116

Tab. 4-16: Schätzergebnisse - Liquidität 117

Tab. 4-17: Signifikanz der Variablen „Liquidität“ 118

Tab. 4-18: Schätzergebnisse - Einlagensicherungssysteme 120

Tab. 4-19: Signifikanz der Variablen „Einlagensicherungssysteme“ 121 
Tab. 4-20: Schätzergebnisse - Einlagensicherungssysteme, ergänzend

Tab. 4-21: Schätzergebnisse - Offenlegungspflicht

Tab. 4-22: Signifikanz der Variablen „Offenlegungspflicht“" 124

Tab. 4-23: Schätzergebnisse - Haftbarkeit Bankmanagement 126

Tab. 4-24: Signifikanz der Variablen „Haftbarkeit“" 127

Tab. 4-25: Schätzergebnisse - Kontrollintensität 128

Tab. 4-26: Signifikanz der Variablen „Kontrollintensität“" 129

Tab. 4-27: Korrelationsmatrix $\quad 130$

Tab. 4-28: Schätzergebnisse - Kontrollintensität, ergänzend 130

Tab. 4-29: Schätzergebnisse - Economic Freedom Indicator 132

Tab. 4-30: Signifikanz der Variablen „Economic Freedom Indicator“ 132

Tab. 4-31: Schätzergebnisse - Ratings 133

Tab. 4-32: Gesamtübersicht der Schätzergebnisse $\quad 137$

Tab. 4-33: Bewertung der Signifikanzverteilung 140

Tab. 4-34: Determinanten der Zinsmargen - Beleg der

Protektionspotentiale durch die Schätzungen 


\section{Abbildungsverzeichnis}

Abb. 2-1: Dienstleistungsexporte 2003 - Aufschlüsselung nach

Dienstleistungsarten

Abb. 2-2: Export von Finanzdienstleistungen - Volumina 23

Abb. 2-3: Export von Finanzdienstleistungen - ausgewählte Länder $\quad 24$

Abb. 2-4: Beispiel eines Members Schedule - das Land ,Arkadien' 26

Abb. 2-5: Struktur der GATS-Verpflichtungen - alle Mitglieder (Modus 1) 27

Abb. 3-1: Determinanten der Zinsmargen 54

Abb. 3-2: Veränderung der absoluten Zinsmargen im Zeitablauf 67

Abb. 3-3: Zinsmargen und Pro-Kopf-Einkommen 70

Abb. 3-4: Vergleich der Zinsmargen (Einkommensklasse > 15.000 US-Dollar) 72

Abb. 3-5: Vergleich der Zinsmargen

(Einkommensklasse 5.000 - 14.999 US-Dollar) 74

Abb. 3-6: Vergleich der Zinsmargen

(Einkommensklasse 1.000 - 4.999 US-Dollar) 76

Abb. 3-7: Vergleich der Zinsmargen (Einkommensklasse $<1.000$ US-Dollar) 78

Abb. 4-1: Aussagekräftigste Schätzgleichung 1998 - 2000

Abb. 4-2: Aussagekräftigste Schätzgleichung $1998-2000$

Abb. 4-3: Aussagekräftigste Schätzgleichung 1998 - 2000, Economic Freedom Gesamtindikator

Abb. 4-4: Aussagekräftigste Schätzgleichung 1998 - 2000, Economic Freedom Gesamtindikator (robuste Schätzung) 155

Abb. 4-5: Aussagekräftigste Schätzgleichung $2001-2003$

Abb. 4-6: Aussagekräftigste Schätzgleichung $2001-2003$

(robuste Schätzung) 


\section{Verzeichnis der Modell-Variablen}

\begin{tabular}{l|l} 
Variable & Bedeutung \\
\hline$B$ & Nettoposition der Bank auf dem Kreditmarkt \\
$C_{D}$ & Verwaltungskosten der Depositen \\
$C_{L}$ & Verwaltungskosten der Kredite \\
$D$ & Depositen, auch Depositenangebotsfunktion \\
$D^{*}$ & ex ante Depositen (Depositenbestand zu Beginn einer Periode) \\
$E$ & Eigenkapital \\
$L$ & Kreditvolumen, auch Kreditnachfragefunktion \\
$R$ & Kapitalmarktzins \\
$R_{D}$ & Depositenzins \\
$R_{L}$ & Kreditzins \\
$P$ & Prämie des Einlagensicherungssystems \\
$Z$ & Risikoprämie \\
& \\
$\pi$ & Gewinn \\
$\mu$ & Zufallsterm
\end{tabular}




\section{Einleitung}

Die Globalisierung der Weltwirtschaft hat im Verlauf der letzten drei Jahrzehnte den internationalen Fluß der Kapitalströme stark intensiviert. Diese Entwicklung wird gemeinhin von ökonomischen und politischen Akteuren begrüßt, weil sie die weltweite Verfügbarkeit des Produktionsfaktors Kapital und damit die Realisierung von Investitionen erleichtert. Einige Nationen, insbesondere die Entwicklungs- und Schwellenländer stehen dieser Entwicklung skeptisch gegenüber, da sie den Verlust politischer Eingriffs- und Kontrollmöglichkeiten fürchten und eine vermeintlich hohe Abhängigkeit von anonymen, mächtigen und nicht kontrollierbaren Finanzinvestoren sehen. In diesem Umfeld gewinnen die Banken in ihrer Funktion als Intermediär von Finanzaktiva zunehmend an Bedeutung. Die Regulierung von Banken und ihrer Aktivitäten stellt für die politischen Akteure einen bedeutenden Ansatzpunkt dar, um wirtschaftspolitischen Einfluß hinsichtlich der Steuerung von Kapitalströmen zu nehmen.

Die Märkte für Bankdienstleistungen weisen, verglichen mit anderen Märkten, einen besonders hohen Regulierungsgrad auf. Die Ursachen hierfür sind in der Verschmelzung zweier Interessen zu finden: Einerseits fußen sie auf dem Bemühen um die Erhaltung der Stabilität und Funktionsfähigkeit des Banken- und Finanzsystems; andererseits basiert der hohe Regulierungsgrad auch auf protektionistischen Ursachen. Marktzugangsbehinderungen gegenüber ausländischen Konkurrenten sichern den inländischen Banken eine privilegierte Wettbewerbsposition. Diesem Umstand verdanken sie Renten, die ihnen unter Bedingungen des Freihandels nicht zufallen würden. So wiesen Claessens, Demirgüc-Kunt und Huizinga in ihrer Studie aus dem Jahr 1998 nach, daß in Entwicklungsländern die Etablierung ausländischer Banken die Gewinnmargen der inländischen Kreditinstitute deutlich reduzierte. Die Protektion des Bankensystems impliziert natürlich auch Kosten. Die durch Protektion bedingten Effizienzverluste würden durch eine stärkere Liberalisierung, wie sie bspw. die Verhandlungsrunden der World Trade Organization (WTO) anstreben, verhindert.

Die Mitgliedsstaaten der WTO beschlossen im Verlauf der Uruguay-Runde (1986 bis 1994), einen separaten Verhandlungsrahmen allen dienstleistungsrelevanten Themen zu widmen. Dies führte zum Abschluß des General Agreement on Trade in Services, kurz GATS, das zum 1.1.1995 in Kraft trat. Ähnlich wie das General Agreement on Tariffs and Trade (GATT) für den Güterhandel soll das GATS die Handelsbedingungen für Dienste dokumentieren und die Basis für die Reduktion von protektionistischen Maßnahmen darstellen. Die derzeit hohen Restriktionen auf den Dienstleistungsmärkten und die unterschiedlichen Vorstellungen über Zeitplan, Art und Ausmaß der Reduzierungen beinhalten ein hohes Konfliktpotential für die bisherigen wie auch noch alle zukünftigen Verhandlungsrunden. 
Von einer Liberalisierung des Handels mit Bankendiensten werden positive Impulse auf verschiedenen Ebenen erwartet: Steigender Konkurrenzdruck veranlaßt die Banken, ihre Geschäftsmodelle auf hinreichende Effizienz und Effektivität zu untersuchen, er erzwingt eine intensive Auseinandersetzung mit den Bedürfnissen und Wünschen der Kunden sowie ein auf sie abgestimmtes Angebot an neuen Diensten. Der Bankenmarkt würde kundenorientierter, effizienter und auch innovativer. Die privaten Haushalte können ihre Ersparnisse zu günstigeren Konditionen anlegen, ihre Renditen steigen. Auch die Unternehmen profitieren von mehr Wettbewerb auf dem Bankenmarkt; sie erhalten ihre Kredite (oder andere Leistungen) zu günstigeren Konditionen. Eine höhere Leistungsfähigkeit des Finanzsektors sollte sich durch die Verbesserung der Investitionsbedingungen insgesamt positiv auf andere Märkte auswirken und den Entwicklungs- und Wachstumsprozeß eines Landes in erheblichem Ausmaß unterstützen (Claessens 2006, 6; Francois / Eschenbach 2002, 10). Des weiteren kann durch den Markteintritt ausländischer Banken der inländischen Wirtschaft Kapital zugeführt werden. Bei investiver Verwendung der Kapitalimporte erhöht sich das Produktionspotential der Volkswirtschaft.

Ein intensivierter Wettbewerb steigert darüber hinaus die Stabilität des Finanzsystems. Banken, die ineffizient arbeiten oder sogar Insolvenzgefahren bergen, werden bei funktionierendem Wettbewerb relativ schnell enttarnt. Sie können den Dienstleitungen und den Preisen der Konkurrenten auf Dauer kein gleichwertiges Angebot entgegensetzen. Die Kreditinstitute sehen sich also dem Druck ausgesetzt, ihre Produkte und Organisationsstrukturen stets aufs Neue zu optimieren.

Im Mittelpunkt dieser Arbeit steht die Untersuchung der Regulierungen der Bankenmärkte und die Identifikation vorhandener Protektionspotentiale bzw. -mechanismen, welche den Kapitalverkehr und die Banken in ihren Aktivitäten beschränken. Die vorherrschende Protektion im Dienstleistungshandel verzerrt analog zum Güterhandel die Marktergebnisse und verursacht Ineffizienzen bspw. in Form von Allokationsverzerrungen und damit Wohlfahrtsverluste. Entsprechend werden im zweiten Kapitel der Arbeit zunächst die grundlegenden Begriffe, die den Hintergrund für die weitere Analyse darstellen, eingeführt. Nach einer allgemeinen Darstellung der Protektion im Welthandel und des internationalen Vertragswerkes GATS zu deren Reduzierung widmet sich das Kapitel den Besonderheiten des Dienstleistungshandels im Bankensektor und den dort auftretenden Hemmnissen.

Im dritten Kapitel wird die Zinsmarge als potentieller Indikator für Protektion erläutert. Sie dient als Gradmesser für das Ausmaß der Protektion. Zinsen übernehmen die Funktion des Preises auf dem Kapitalmarkt. Allerdings ist dieser nicht einheitlich. Die Banken entrichten als Intermediär von Finanzaktiva für Depositen einen geringeren Zinssatz als sie ihn für die Gewährung von Krediten verlangen. Diese Zinsdifferenz, normiert auf die zinstragenden Vermögenswerte, ist die (Netto-)Zinsmarge. Sie repräsentiert die verschiedenen Aspekte der Bankenmarkt- 
struktur. Neben der Funktion die Betriebskosten zu decken, spiegelt die Zinsmarge auch die institutionellen und ökonomischen Rahmenbedingungen sowie die aktuell vorherrschende Protektion wider. Wird die Preisbildung durch Protektion verzerrt, so richten die Marktteilnehmer ihre Transaktionen aber an einem „falschen“ Signal aus. Daraus folgen Fehlallokationen des Produktionsfaktors Kapital.

Neben der konzeptionellen Idee der Zinsmargen wird im dritten Kapitel auch das sog. erweiterte Dealer-Modell erläutert. Es erklärt, wie die Banken ihre jeweils optimalen Zinshöhen ermitteln, die sich zwischen den Ländern, aber auch zwischen den Banken eines Landes unterscheiden. Anhand des Modells lassen sich die Wirkungsweisen protektionistischer Regulierungen erläutern und wie sie in das Kalkül der Banken einfließen. Den Abschluß des Kapitels bildet die Darstellung und quantitative sowie qualitative Interpretation der zur Verfügung stehenden Zinsmargen-Daten.

Das vierte Kapitel widmet sich der Regressionsanalyse der Zinsmargen und ihrer Determinanten. Ziel ist die Ermittlung von Einflußfaktoren, die bereits protektionistisch eingesetzt werden (bspw. Marktstruktur, administrative Vorschriften und weitere Regulierungen). Durch die empirische Analyse wird geklärt, welche Faktoren die Zinsmarge generell determinieren und welche dieser Variablen Instrumente der Protektion darstellen. Hierfür wird die Zinsmarge auf die verschiedensten Bedingungen der Bankenmärkte regressiert. Da Daten aus zwei Zeiträumen zur Verfügung stehen, findet ein Vergleich der Determinanten statt, um eventuelle Veränderungen ihrer Bedeutung oder ihrer Verwendung als Protektionsinstrumente im Zeitablauf zu ermitteln. Einige der Determinanten der Zinsmargen bieten Ansatzpunkte für eine protektionistische Wirtschaftspolitik: Zunächst die Determinanten der Zinsmargen und in einem zweiten Schritt die protektionsrelevanten Determinanten herauszuarbeiten, stellt den Kern dieser Arbeit dar. Ihre exakte Identifizierung ist von Bedeutung für weitere Deregulierungsbemühungen im Rahmen der GATS-Verhandlungen. Auf diese Weise können effektive Liberalisierungen durchgeführt werden und Zeitverluste durch den Abbau nur scheinbar restriktiver Bestimmungen verhindert werden. Im Hinblick auf diesen Aspekt werden die Ergebnisse der Regressionen eingehend analysiert. Anschließend werden die beiden aussagekräftigsten Schätzgleichungen für beide Untersuchungszeiträume vorgestellt und wirtschaftspolitische Schlußfolgerungen gezogen.

Den Abschluß der Arbeit bildet ein kurzes Fazit, das auch Hinweise auf noch offene Fragestellungen enthält, die im Rahmen dieser Arbeit nicht geklärt werden konnten. 


\section{Internationaler Dienstleistungshandel}

\subsection{Protektionismus und GATS}

\subsubsection{Protektion im Welthandel}

Die Analyse des internationalen Dienstleistungshandels und seiner zahlreichen Beschränkungen erfordert die Betrachtung der vorherrschenden Protektionspraktiken sowie eines Instruments zum Regulierungsabbau, des General Agreement on Trade in Services (GATS).

Unter dem Begriff Protektion werden alle Maßnahmen subsumiert, die auf die Bevorzugung und den Schutz inländischer Unternehmen gegenüber ausländischer Konkurrenz abzielen. ${ }^{1}$ Die Motive für die Begünstigung der inländischen Wirtschaft sind vielfältig. Es wird argumentiert, daß der Schutz moderner oder bestimmter Industrien sowie der Erhalt heimischer Arbeitsplätze (trotz ihrer möglichen Unrentabilität) von hoher politischer und ökonomischer Bedeutung sind. Als Rechtfertigung für protektionistische Eingriffe dienen auch Argumente wie die Sicherung von Marktanteilen inländischer Firmen, die Unterstützung bei der Eroberung von Auslandsmärkten oder die Erhöhung der Wettbewerbsfähigkeit einzelner Unternehmen. Das Wiederwahl-Interesse der Politiker steigert die Empfänglichkeit des politischen Apparates für derlei von Lobbyisten vorgebrachte Argumente. Da sich Konsumenten im Gegensatz zu einzelnen Branchen schlecht organisieren lassen sowie Verbraucher die Folgen der Protektion nicht klar erkennen können (Informationsasymmetrie), fällt der Widerstand gegen Protektionswünsche der Wirtschaft in der Regel eher gering aus (Ellingsen / Wärneryd 1999, 357 f.). Es ist davon auszugehen, daß der Markt für Protektion wohl auch in Zukunft florieren wird. Die gestaltenden und ausführenden Organe der Protektion sind die Regierungen und ihre Administrationen, wenn auch aus politökonomischer Sicht letztlich Interessengruppen als Initiatoren auftreten (Rosen / Windisch 1992, 199 ff.). ${ }^{2}$

1 Hinsichtlich konkreter Definitionen des Protektionsbegriffs sei verwiesen auf Geigant u.a. (2000, 800): „Maßnahmen der Außenwirtschaftspolitik zum Schutz inländischer Sektoren bzw. Wirtschaftssubjekte. Im Bereich der Außenhandelspolitik werden protektionistische Maßnahmen ergriffen, um die Verteilungsposition von durch Freihandel geschådigten Faktoren zu verbessern (Samuelson-Stolper-Theorem), außenwirtschaftlich verursachte Strukturanpassungen zu erleichtern, die inländische Beschaftigung zu stimulieren und die Leistungsbilanz zu verbessern, die Entwicklung komparativer Vorteile (Optimalzoll-Theorie) zu erzielen.“ Oder Walter (1972): ,....any measure that distorts the volume of trade, the composition of the basket of goods traded between countries or the direction in which goods are traded".

2 Auf die politokonomischen Grunde für die Entstehung und das Funktionieren von Protektionsmarkten wird im Rahmen dieser Arbeit nicht weiter eingegangen; es sei auf WeckHannemann (1992) verwiesen. 
Das klassische Instrument der Protektion ist die Zollerhebung. Da dieses im Dienstleistungsbereich nicht anwendbar ist, ${ }^{1}$ kommt den nicht-tarifären Handelshemmnissen (Non-Tariff Barriers, NTB) ein hoher Stellenwert zu. Unter dem Begriff der NTB werden alle Handelsbarrieren zusammengefaßt, die nicht Zölle sind. ${ }^{2}$ Wie aus der Außenhandelstheorie bekannt, sind die Folgen protektionistischer Maßnahmen weit gestreut. Die Effekte der Protektion lassen sich aus der klassischen Zolltheorie ${ }^{3}$ ableiten, deren Ergebnisse analog auf die Wirkungen anderer Handelshemmnisse übertragen werden können. Durch die Einführung von Zöllen oder NTB's steigen zunächst die Inlandspreise für Importgüter. Die Nachfragemengen sinken und die Versorgung der inländischen Bevölkerung mit diesem Gut verschlechtert sich, was einem inländischen Wohlfahrtsverlust entspricht. Des weiteren ergeben sich Umverteilungen der Renten zugunsten der inländischen Produzenten und zulasten der inländischen Konsumenten wie auch der ausländischen Produzenten. Insgesamt sinkt die Weltwohlfahrt. Der Schutz eines Sektors liefert durch die Verzerrung der Preise falsche Signale an die Märkte. Produktionsfaktoren richten sich an diesen aus und werden nicht mehr in die effizientesten Produktionen gelenkt, sondern relativ stark auf die protektionierten Branchen konzentriert.

In einer Welt ohne Handelshemmnisse hingegen können die Vorteile der internationalen Arbeitsteilung und Spezialisierung gemäß komparativer Vorteile genutzt und somit die (Welt-)Wohlfahrt maximiert werden. Jegliche Einschränkung dieses Prozesses durch Hemmnisse ist mit einer Verringerung der Wohlfahrt gleichzusetzen, auch wenn temporär nationale Wohlfahrtserhöhungen durch strategisches Verhalten erlangt werden können. Diese Argumentation erklärt das Eintreten der WTO für freie Handelsbeziehungen; es ist letztlich ein Programm zur Erhöhung der Wohlfahrt aller Nationen.

Die Handelshemmnisse im Dienstleistungs- und Warenbereich unterscheiden sich erheblich. So sind die Märkte für Dienstleistungen in der Regel von rigideren und höheren Handelsbarrieren geprägt als die Gütermärkte. Handelsregulierungen sind in den Entwicklungsländern zumeist stärker ausgeprägt als in den Industrieländern. Auch sind die verschiedenen Dienstleistungsbereiche unterschiedlich stark von den Regulierungen hinsichtlich ihrer Handelbarkeit betroffen. So treten sie in den Sektoren Transport, Finanzen und Telekommunikation häufiger auf als bei Distributionsdienstleistungen und unternehmensbezogenen Dienstleistungen (Hoekman 2000, Findlay / Warren 2000, Robinson / Wang / Martin 2002). Nach einer Schätzung des IMF in Kooperation mit der Weltbank würde der Abbau dieser Handelshemmnisse zunächst mit eher bescheidenen statischen, aber mittel-

1 Vgl. hierzu die năheren Ausfuhrungen in Kapitel 2.2.1 Banken als Dienstleistungsunternehmen.

2. Weitere Ausfuhrungen hierzu im Kapitel 2.2.3 Hemmnisse im Dienstleistungshandel.

3 Hierfur sei auf gängige Lehrbucher verwiesen wie Krugman/Obstfeld (2003, 186 ff.) oder Maennig/Wilfling (1998, $151 \mathrm{ff}$.). 
bis langfristig mit hohen dynamischen Liberalisierungsgewinnen belohnt (IMF / World Bank 2001, 44). Diese speisen sich vor allem aus der Reallokation der Produktionsfaktoren sowie aus der Anregung von Investitionen, aus Produktivitätssteigerungen und der verbesserten Nutzung von Skaleneffekten. Je stärker der liberalisierte Sektor ursprünglich geschützt wurde - und der Finanzsektor ist ein besonders stark regulierter Wirtschaftsbereich -, um so höhere Gewinne resultieren aus Deregulierung und Marktöffnung. Darüber hinaus ist erwiesen, daß Länder, die ein stark wettbewerbsorientiertes Bankensystem aufweisen, ein höheres Niveau an Wirtschaftsaktivität erreichen und nicht so schwere Konjunkturzyklen hinnehmen müssen (Smith 1998, 794). Das Instrument des Protektionsabbaus im internationalen Dienstleistungsverkehr, das GATS, wird im Folgenden eingehend vorgestellt.

\subsubsection{Das GATS als Instrument zur Protektionsreduzierung}

Der Dienstleistungshandel hat seit Mitte der 1980er Jahre wertmäßig stark zugenommen und umfaßt ca. ein Fünftel des Welthandels, mit steigender Tendenz (Matthes 2001, 32 ff.). Da Dienstleistungen aufgrund ihrer überwiegend immateriellen Natur einige Besonderheiten ${ }^{1}$ im Handel aufweisen, wurden sie nicht in bereits bestehende WTO-Abkommen integriert; ihnen wurde mit der Ratifizierung des GATS ein eigenständiger juristischer Rahmen zugestanden. Die Einigung auf dieses Vertragswerk gilt als wichtigster Erfolg der Verhandlungen der sog. Uruguay-Runde, die ihren Abschluß im Jahr 1996 fanden (Sapir 1999). Die Schaffung des GATS erlaubt die systematische Identifikation der Handelskonditionen für Dienstleistungen. Die Offenlegung aller Handelshemmnisse schuf eine enorme Transparenz. Damit wurde gleichzeitig die Grundlage für Verhandlungen über ihre Reduzierung gelegt.

Die im GATS verwendete Definition von Dienstleistungen ist sehr allgemein formuliert (WTO 1995):

„... 'services' includes all services in any sector except services supplied in the exercise of governmental authority."

Die enumerative Erfassung der Dienstleistungen wurde bevorzugt. Die Mitgliedsstaaten der WTO einigten sich als Verhandlungsgrundlage auf eine konkrete Klassifizierung (GNS-Classification $\left.{ }^{2}\right)$. Sie besteht aus 12 Hauptkategorien und 155 Unterkategorien und soll eine überschneidungsfreie Zuordnung der Dienstleistungen ermöglichen (Krancke 1999, 8).

Auf die Eigenschaften von Dienstleistungen geht Kapitel 2.2.I Banken als Dienstleistungsunternehmen detailliert ein.

2 Die WTO setzte die Arbeitsgruppe Group of Negotiations in Services (GNS) ein, deren Aufgabe in der Schaffung einer Systematik zur Erfassung der Dienstleistungen lag. Sie basiert auf der umfangreichen, in vier Ebenen strukturierten Central Product Classification (CPC). 
Die 12 Hauptkategorien umfassen:

- unternehmensbezogene Dienste (Rechtsanwälte, Notare, Forschung etc.),

- Kommunikationsdienstleistungen (Post, Telekommunikation, Internet),

- Bau- und Montagedienstleistungen,

- Vertriebsdienstleistungen (Groß- und Einzelhandel),

- Bildungsdienstleistungen (Schulen, Universitäten),

- Umweltdienstleistungen (Recycling, Entsorgung),

- Finanzdienstleistungen (Banken, Versicherungen),

- medizinische und soziale Dienstleistungen (Krankenhäuser, Altenpflege),

- Tourismus und Reisen (Hotels, Reiseveranstalter),

- Erholung, Kultur, Sport (Theater, Büchereien, Freizeitaktivitäten),

- Transportdienstleistungen (Land, Wasser und Luft) sowie

- sonstige, nicht aufgeschlüsselte Dienstleistungen.

Der Illustration der Bedeutung der einzelnen Dienstleistungsarten im internationalen Handel dient folgende Graphik. Die herausragende Bedeutung der unternehmensbezogenen Dienstleistungen im internationalen Handel ist deutlich zu erkennen. Ebenso ist auch die unterschiedliche Handelsstruktur in den drei untersuchten Regionen Nordamerika, Europa und Asien ersichtlich. Während der Export von Finanzdiensten besonders bedeutend für Europa (14 v.H. der gesamten Dienstleistungsexporte) und auch Nordamerika (10 v.H.) ist, fällt Asien hingegen in dieser Kategorie mit einem Anteil von nur 7 v.H. auf dem Weltmarkt zurück. 

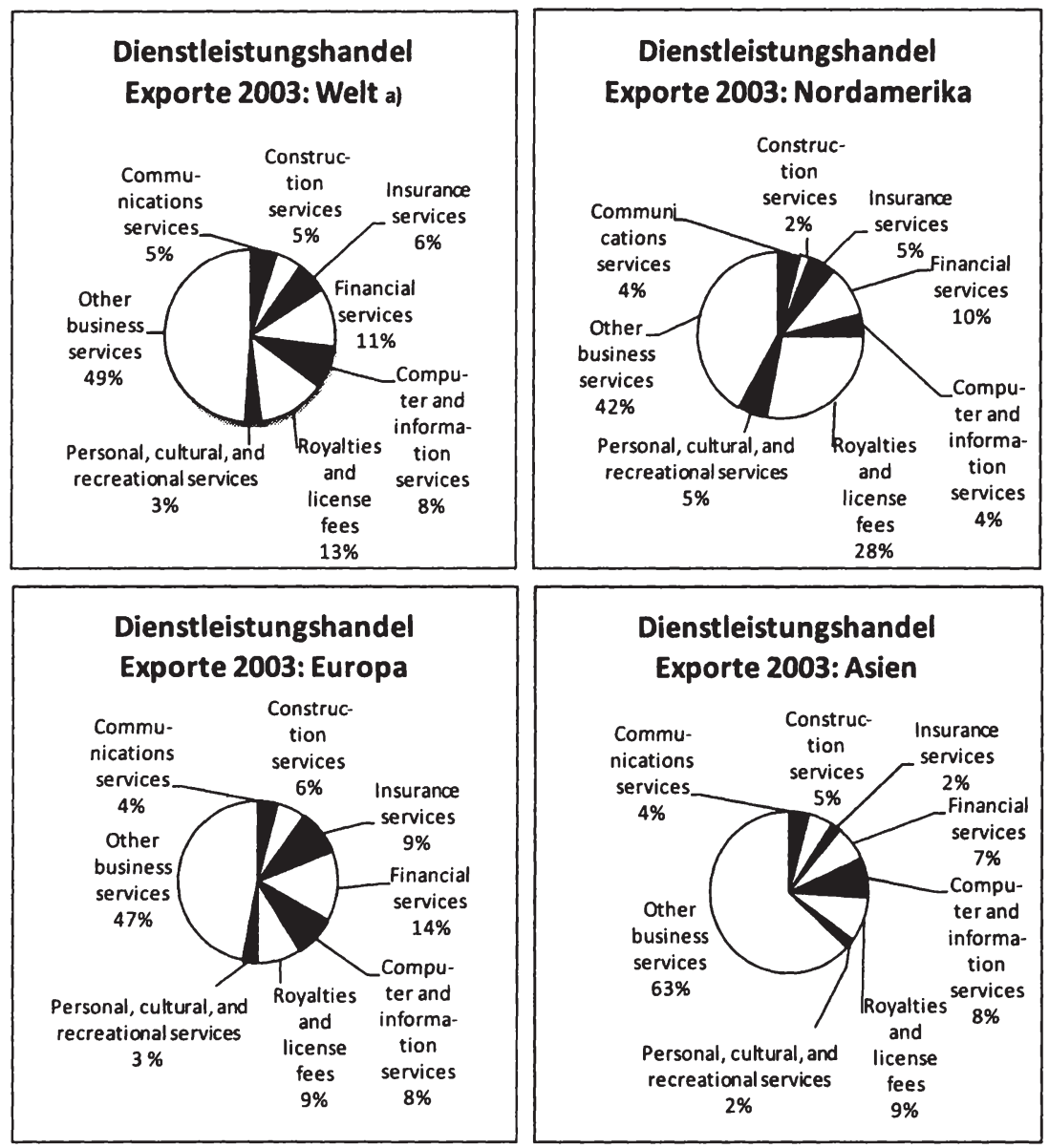

a) Ohne den Intra-EU (15) Handel.

Quelle: WTO, die Daten wurden im Rahmen einer Anfrage per E-Mail bei der Statistischen Abteilung der WTO im März 2006 ermittelt.

Da sich die Arbeit insbesondere mit den international gehandelten Finanzdiensten beschäftigt, werden ergänzend deren weltweite Exportdaten angegeben. 
Abb. 2-2: Export von Finanzdienstleistungen - Volumina

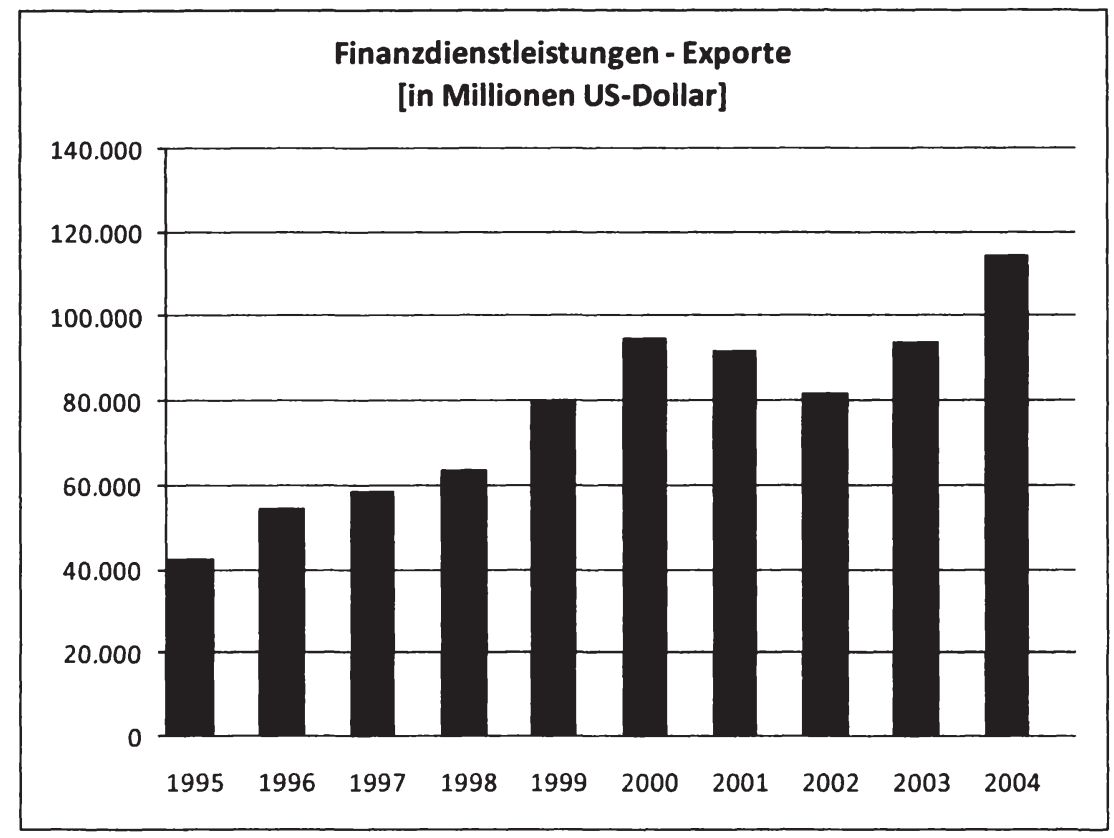

Quelle: WTO, die Daten wurden im Rahmen einer Anfrage per E-Mail bei der Statistischen Abteilung der WTO im März 2006 ermittelt.

Die rasante Zunahme des Handels mit Finanzdiensten ist gut zu erkennen. Innerhalb von nur 9 Jahren hat sich das Handelsvolumen annähernd verdreifacht und die positive Tendenz trotz der großen Finanzkrise in den Jahren 2001 / 2002 beibehalten können.

Die folgende Graphik verdeutlicht die unterschiedliche Teilhabe der Länder am Handel mit Finanzdiensten. Während die Industrienationen hohe Volumina verzeichnen, sind Entwicklungsländer nur mit einem geringen Anteil an den weltweit getätigten Exporten beteiligt. Dies wird deutlich anhand der unterschiedlichen Skalierung der Graphiken. 


\section{Abb. 2-3: Export von Finanzdienstleistungen - ausgewählte Länder}
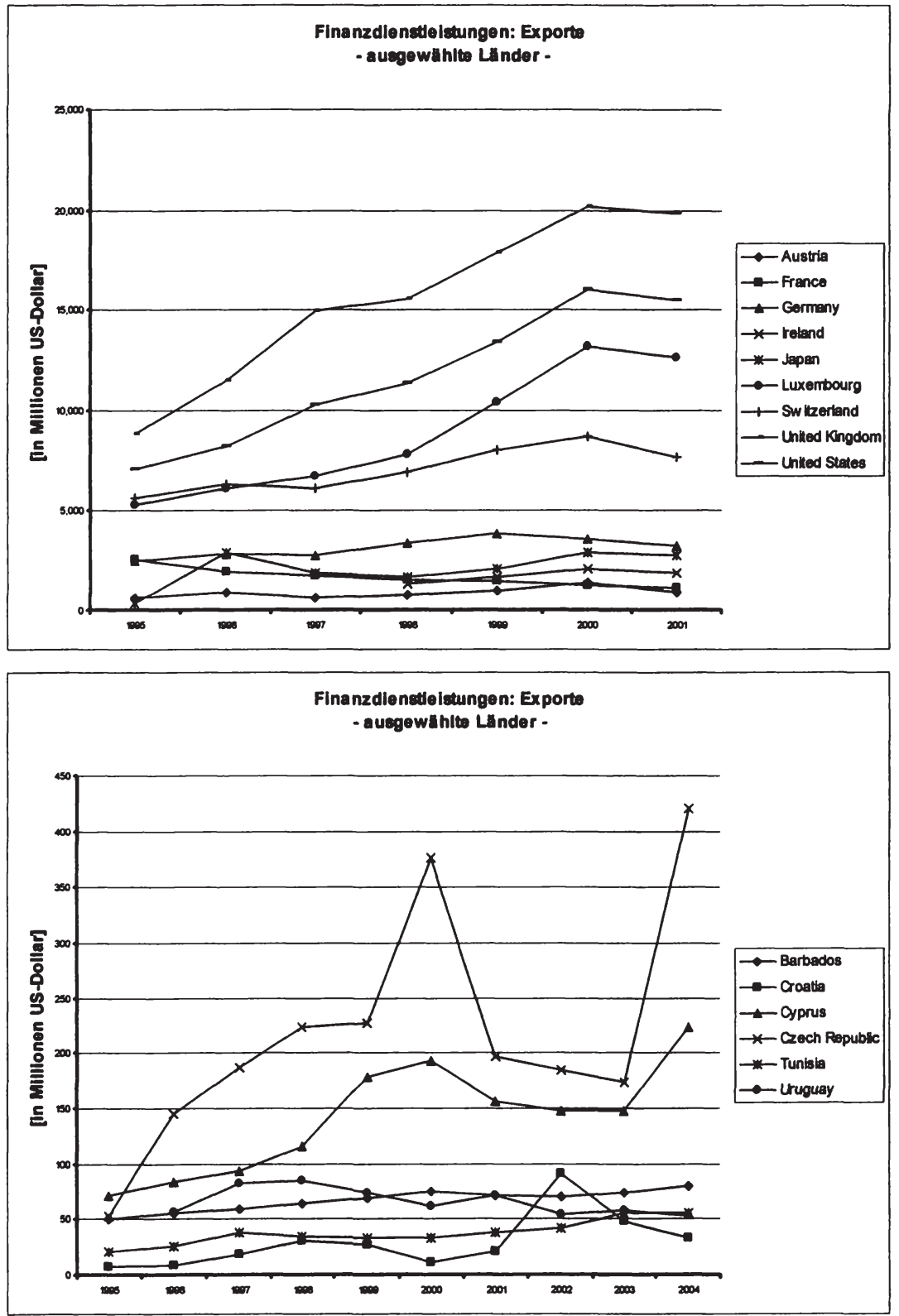

Quelle: WTO, die Daten wurden im Rahmen einer Anfrage per E-Mail bei der Statistischen Abteilung der WTO im März 2006 ermittelt. 


\subsubsection{Grundlegende Prinzipien des GATS}

Das Vertragswerk GATS besteht inhaltlich aus drei Hauptelementen. ${ }^{1}$ Ein Rahmenwerk aus allgemeinen Prinzipien regelt den generellen Umgang der Vertragspartner miteinander, analog zum GATT. Des weiteren besteht das Vertragswerk aus Anhängen (Annexes) für sensible Sektoren. Darunter werden Regelungen für die Bereiche Lufttransport, Finanzen, maritime Transporte wie auch hinsichtlich der Bewegungsfreiheit natürlicher Personen (Einreise- und Aufenthaltsbestimmungen) subsumiert. Der dritte Teil des Vertragswerkes ist der sog. Schedule of Commitment, auch Members Schedule genannt. Dieser führt die einzelnen Restriktionen bezüglich Marktzugang und Gleichbehandlung von in- und ausländischen Unternehmen (National Treatment) auf. Der Schedule of Commitment verfolgt den Ansatz der Positivliste (Mattoo / Wunsch 2004, 2). Jedes Land benennt die Dienstleistungskategorien, in denen ausländische Anbieter auf dem heimischen Markt agieren dürfen. Es können in einem weiteren Schritt die exakten Bedingungen für den Marktzugang, die Arten der Aktivitäten, konkrete Geund Verbote und weitere Regelungen angegeben werden. In der Gestaltung dieser Bedingungen ist jedes Land völlig frei. Erfolgen keinerlei Angaben zu einem Dienstleistungsbereich, so ist ausländischen Unternehmen die Betätigung in dieser Sparte vollständig untersagt.

Das GATS umfaßt alle Handelsbedingungen, die für Dienstleistungen gelten, ausgenommen sind einzig hoheitliche Aufgaben (z.B. Regelung und Überwachung des Luftverkehrs). Als Beispiel für einen Members Schedule sei das Land ,Arkadien' mit seinen Konditionen für Distributionsdienste angeführt. Die Zahlen 1), 2), 3) oder 4) kennzeichnen die Erbringungsart, d.h. die Lieferweise; für jede einzelne können konkrete Bedingungen formuliert werden. ${ }^{2}$

1 Der Vertrag gliedert sich tatsăchlich in 5 Kapitel: Kapitel I: Generelle Konzepte, Kapitel II: Allgemeine Verpflichtungen bezlglich des grenzuberschreitenden Handels, Kapitel III: Spezielle Verpflichtungen, Kapitel IV: Institutionelle Vorkehrungen und Vorschriften, Kapitel V: Finale Vorschriften, zusătzlich: verschiedene Anhănge (zumeist sektoraler Art) und ministeriale Erklărungen.

2 Die Erbringungsarten werden in Kapitel 2.2.2.1 Formen des Handels năher erlăutert. 
Abb. 2-4: Beispiel eines Members Schedule - das Land ,Arkadien'

\begin{tabular}{|c|c|c|c|}
\hline $\begin{array}{l}\text { Sector or Sub- } \\
\text { sector }\end{array}$ & $\begin{array}{l}\text { Limitations on } \\
\text { Market Access }\end{array}$ & $\begin{array}{c}\text { Limitations on } \\
\text { National Treatment }\end{array}$ & $\begin{array}{l}\text { Additional } \\
\text { Comments }\end{array}$ \\
\hline \multicolumn{4}{|c|}{ I. Horizontal Commitments } \\
\hline $\begin{array}{l}\text { All Sectors } \\
\text { included in this } \\
\text { Schedule. }\end{array}$ & $\begin{array}{l}\text { 4) Unbound }{ }^{\text {a) }} \text { other } \\
\text { than for } \\
\text { (a) temporary } \\
\text { presence, as in } \\
\text { intra-corporate } \\
\text { transferees, of } \\
\text { essential senior } \\
\text { executives and } \\
\text { specialists and } \\
\text { (b) presence for up } \\
\text { to } 90 \text { days of } \\
\text { representatives } \\
\text { of a service } \\
\text { provider to } \\
\text { negotiate sales } \\
\text { of services. }\end{array}$ & $\begin{array}{l}\text { 3) Authorization is } \\
\text { required for } \\
\text { acquisition of } \\
\text { land by } \\
\text { foreigners. }\end{array}$ & \\
\hline \multicolumn{4}{|c|}{ II. Sector-specific Commitments } \\
\hline $\begin{array}{l}\text { 4. Distribution } \\
\text { Services } \\
\text { C. Retailing } \\
\text { Services (CPC } \\
631,632)\end{array}$ & $\begin{array}{l}\text { 1) Unbound } \\
\text { (Except for } \\
\text { mail order: } \\
\text { none). } \\
\text { 2) None }{ }^{\text {b) }} \text {. } \\
\text { 3) Foreign equity } \\
\text { participation } \\
\text { limited to } 51 \\
\text { per cent. } \\
\text { 4) Unbound. }\end{array}$ & $\begin{array}{l}\text { 1) Unbound } \\
\text { (Except for mail } \\
\text { order: none). } \\
\text { 2) None. } \\
\text { 3) Investment } \\
\text { grants are } \\
\text { available only to } \\
\text { companies } \\
\text { controlled by } \\
\text { Arcadian } \\
\text { nationals. }\end{array}$ & \\
\hline
\end{tabular}

a) Unbound = there are no binding commitments.

b) None $=$ no limitations are imposed.

Quelle: WTO, http://www.wto.org/english/tratop_e/serv_e/cbt_course_e/c2sp6p2_e.htm,vom

Jedes Land bestimmt und formuliert selbständig, welche konkreten Vorschriften es für die 12 Hauptkategorien an Dienstleistungen und ihre 155 Unterkategorien 
erlassen will, entsprechend umfangreich sind die Anhänge des GATS. In dem Members Schedule werden horizontal wirkende Verpflichtungen benannt, d.h. Regelungen definiert, die für alle Sektoren gleichermaßen gelten. Für jede Dienstleistung können zusätzlich noch spezifische Regulierungen oder Verbote, separat für jede der vier Erbringungsarten, verfügt werden.

Der Vergleich der Zugeständnisse der Länder im Finanzdienstleistungsbereich mit denen einer Branche, die einen höheren Offenheitsgrad aufweist wie bspw. die Produzentendienste (Datenverarbeitung, Implementierung von Software etc.), verdeutlicht das hohe Ausmaß der vorherrschenden Protektion.

Abb. 2-5: Struktur der GATS-Verpflichtungen - alle Mitglieder (Modus 1)

\begin{tabular}{|c|c|c|c|c|c|c|c|}
\hline \multirow{2}{*}{ Sector } & \multirow{2}{*}{ Total $^{\mathrm{a}}$} & \multicolumn{3}{|c|}{ Market Access (\%) } & \multicolumn{3}{|c|}{ National Treatment (\%) } \\
\hline & & Full & Partial $^{b}$ & Unbound & Full & Partial & Unbound \\
\hline \multicolumn{8}{|l|}{ Financial Services } \\
\hline $\begin{array}{l}\text { Insurance } \\
\text { Intermediation }\end{array}$ & 61 & 18 & 52 & 30 & 45 & 20 & 35 \\
\hline $\begin{array}{l}\text { Lending of all } \\
\text { Types }\end{array}$ & 93 & 24 & 35 & 41 & 42 & 18 & 40 \\
\hline \multicolumn{8}{|l|}{ Business Services } \\
\hline $\begin{array}{l}\text { Software } \\
\text { Implementation }\end{array}$ & 71 & 61 & 24 & 15 & 65 & 17 & 18 \\
\hline $\begin{array}{l}\text { Data Processing } \\
\text { Services }\end{array}$ & 69 & 59 & 25 & 16 & 62 & 20 & 17 \\
\hline
\end{tabular}

= Total number of members with commitments in mode 1, the cross-border supply.

$\mathrm{b}=$ Includes horizontal limitations.

Quelle: Mattoo / Wunsch (2004, 16).

Der vollständige Marktzugang (full market access) wird ausländischen Unternehmen bei den Finanzdiensten nur zu einem sehr geringen Prozentsatz gewährt (18 v.H. im Versicherungsbereich bzw. 24 v.H. im Kreditbereich). Bei den Produzentendiensten reicht die Freizügigkeit sehr viel weiter: 61 v.H. bzw. 59 v.H. der Länder gewähren diesen Diensten freien Marktzugang.

„Unbound" kennzeichnet die für Ausländer nicht freigegebenen Tätigkeitsbereiche. Gerade hier wird deutlich, wie groß der Liberalisierungsbedarf ist. Den Marktzugang für ausländische Unternehmen im Versicherungsbereich verwehren 30 v.H. der Länder, im Kreditbereich sogar 41 v.H. Die Gleichbehandlung mit inländischen Unternehmen (National Treatment) verwehrt ein ähnlich hoher Prozentsatz der Länder: im Versicherungsbereich sind es 35 v.H., im Kredit- 
bereich sogar 40 v.H. Für die Unternehmen der elektronischen Datenverarbeitung sind diese Prozentsätze sehr viel niedriger, dort bewegen sie sich in der Bandbreite von 15 v.H. bis 18 v.H.

Grundsätzlich gelten für den Dienstleistungshandel dieselben Prinzipien, die bereits seit fünf Dekaden beim GATT verwendet werden und dort im Zeitablauf zu einer erheblichen Verringerung von Zöllen und zollähnlichen Handelshemmnissen beigetragen haben.

Die zentralen Prinzipien lauten (OECD 2002, 57):

a) Gleichbehandlung von in- und ausländischen Anbietern (National Treatment),

b) Meistbegünstigungsprinzip (Most-Favoured Nation-Principle, MFN),

c) Transparenz der heimischen Regulierungen,

d) Angemessene, d.h. nicht diskriminierende Anwendung der Gesetze.

Die Gleichbehandlung von in- und ausländischen Anbietern soll Diskriminierung, die allein im Herkunftsland begründet liegt, verhindern. So soll eine Benachteiligung ausländischer Unternehmen z.B. durch inländische Behörden bei Verwaltungsakten oder durch politische Akteure ausgeschlossen werden.

Das Meistbegünstigungsprinzip erfordert von allen Vertragspartnern, daß die einem Land gewährten Handelsvorteile grundsätzlich auch auf alle anderen Handelspartner übertragen werden müssen. Das GATS erlaubt Ausnahmen von dieser Regel, und von dieser Möglichkeit machen auch rund 2/3 der WTO-Länder Gebrauch. Die Ausnahmeregelungen sollen maximal 10 Jahre andauern, allerdings wurde diese Zeitbeschränkung in 4 von 5 Fällen nicht angegeben, und somit bleiben sie unbegrenzt bzw. bis zur Nachverhandlung in Kraft (Matthes 2001, 33).

Die Transparenz der Bedingungen, zu denen ausländische Unternehmen den Inlandsmarkt bedienen dürfen, wird durch die ausführlichen Angaben im Members Schedules gewährleistet. Das GATS verpflichtet die Länder, in den gelisteten Dienstleistungssektoren alle Regulierungen und Bestimmungen offenzulegen und stets auf dem neuesten Stand zu halten. Damit wird das GATS zu einer bedeutenden Informationsquelle für Regierungen und Unternehmen.

Ein verläßliches und funktionierendes Gerichtssystem soll ausländischen Unternehmen das Recht sichern, ihre Ansprüche notfalls auch juristisch durchzusetzen. Damit sollen Benachteiligungen im Geschäftsverkehr oder Enteignungen verhindert werden.

Vgl. hierzu WTO-Homepage: http://www.wto.org/english/tratop_e/serv_e/gats_factfiction6_ e.htm, vom 29. Mărz 2006. 


\subsubsection{Charakteristika und Defizite des GATS}

Während das GATT sich nur auf Produkte bezieht, wird im GATS der Begriff des "Sektors" benutzt wie auch die Formulierung "Services and Service Supplier". Damit ist der Gültigkeitsbereich der GATS-Bestimmungen sehr viel weiter gefaßt. Die Hoffnung, daß der Sektorenansatz gut geeignet sei, Liberalisierungserfolge auf ganze Bereiche zu erweitern und langwierige Verhandlungen über einzelne Dienstleistungen zu vermeiden, hat sich jedoch nicht erfüllt (Sapir 1999, 56). In der Bewertung dieses sektoralen Ansatzes des GATS herrscht in der akademischen Diskussion mittlerweile eher Ernüchterung vor. Die politische Einflußnahme bedeutender Sektoren durch Lobbyisten auf die Verhandlungsdelegationen blieb erhalten; die Deregulierungsverhandlungen verlaufen entsprechend eher schleppend.

Nationale Regulierungen sind unter den GATS-Bestimmungen weiterhin möglich und werden auch nicht beschränkt (Djordjevic 2002, 305 sowie 307). Nur die Behinderungen des Marktzugangs oder die Diskriminierung ausländischer Unternehmen sind - zumindest theoretisch - ausgeschlossen. Regierungen dürfen also durchaus die bestehenden Regulierungen ihrer Märkte beibehalten oder neue etablieren. Diese müssen aber veröffentlicht werden und innerhalb eines Jahres dem Rat für Dienstleistungshandel gemeldet werden (Sapir 1999, 54 f.). Die Länder sind verpflichtet, Informationsbehörden einzusetzen, die hinsichtlich aller für einen Sektor geltenden handelsbeschränkenden Bestimmungen Auskunft erteilen. Diese Regelung dient der Erhöhung der Transparenz und reicht weit über die Anforderungen des GATT hinaus. Daraus ergeben sich aber auch Änderungen in der Zusammensetzung der Verhandlungsdelegationen. Während die Teilnahme bei GATT-Verhandlungen auf Bevollmächtigte für Handelsfragen beschränkt blieb, sind bei GATS-Verhandlungen sowohl Beamte für Handelsfragen als auch Mitarbeiter der Regulierungsbehörden involviert. Komplexe interpersonelle Verhandlungen bergen die Gefahr eines hohen protektionistischen Beharrungsvermögens, da sie zahlreiche Abstimmungen erfordern und die Kompromißbildung erschweren. Sie tragen damit nicht zu einem effizienten und schnellen Abschluß der Gespräche bei.

Die Verhandlungsregelungen sind ebenfalls nicht unproblematisch. Die Diskussion einzelner Handelskonditionen erfolgt nur auf direkte Anforderung hin (request-and-offer-approach) und nur von einem Land zum anderen hin sowie Sektor für Sektor. Dieses Verfahren ist umständlich, wenig effektiv und sehr zeitaufwendig (Poveda / Droege 1997, 625). Deshalb muß von wissenschaftlicher Seite die Forderung nach einem Übergang zu multilateralen Verhandlungen erhoben werden.

Problematisch im Rahmen des GATS gestaltet sich auch die eingeschränkte Wirksamkeit der Meistbegünstigungsklausel. Der häufige Gebrauch von einzelstaatlichen Regulierungen und verzeichneten Ausnahmen im Members Schedule 
fördert den intensiven Gebrauch dieses Instrumentes, um Märkte vor ausländischer Konkurrenz zu schützen. Während der Uruguay-Verhandlungen wurden insbesondere in den Bereichen Finanzdienstleistungen und hochwertige Telekommunikationsdienstleistungen hohe Marktzugangsbarrieren errichtet (Sapir 1999, 56).

Das System der Positivlisten bietet den Ländern großzügige Spielräume. Es werden nicht die Bereiche aufgeführt, die noch reguliert sind und deren Liberalisierung zu verhandeln wäre (Negativlisten), sondern die Dienstleistungsbranchen gemeldet, die ausländischen Unternehmen zur Betätigung offenstehen bzw. deren Bestimmungen sie einzuhalten haben. Das System der Positivliste verschlechtert die Verhandlungschancen im Hinblick auf zuküntige Liberalisierungen, denn jedes Land hat das Recht, Dienstleistungsbereiche nach eigenem Gutdünken aus den Verhandlungen auszuschließen (Krancke 1999, 27). Außerdem erfaßt die Positivliste Innovationen im Dienstleistungsbereich immer erst mit erheblicher zeitlicher Verzögerung. Offiziell verhandelbar sind aber nur die Konditionen für bereits erfaßte Dienste. Die Einigung auf Kriterien für die Erfassung von Dienstleistungen und die automatische Aufnahme aller neu hinzukommenden Dienstleistungen würde den Definitions- und Verhandlungsbedarf der Länder erheblich reduzieren.

Ein grundsätzliches Problem bei Dienstleistungen ist, daß sie teilweise in die politisch-sozialen Konstellationen und Strukturen eines Landes hineinreichen. In Abhängigkeit vom politischen System und historisch gewachsenen Strukturen vertreten die Länder unterschiedliche Auffassungen, welche Dienste von staatlicher Seite angeboten werden sollen. Dieser Umstand wirkt sich u.a. auf das Sozialwesen, die Bankenaufsicht, das Ausmaß staatlicher Aktivitäten, die Rechtsordnung und die Regulierungsdichte aus. Diese Bereiche zu liberalisieren, trifft bei vielen Ländern auf erheblichen Widerstand.

Lösungsvorschläge im Hinblick auf eine Verbesserung des GATS wie auch zur weitergehenden Liberalisierung sind vielfältig, z.B. sollten die Verhandlungen auf horizontaler Ebene belassen werden. Damit würden Regulierungen für eine ganze Klasse von Dienstleistungen verhandelt und nicht für jeden Dienst einzeln die exakten Bestimmungen diskutiert werden. Auch sollten vorrangig die Verhandlungen auf Sektoren konzentriert werden, die als Zulieferer für weitere Wirtschaftszweige bedeutend sind. Der Wechsel von der Positivliste zur Negativliste wird dringend empfohlen. Damit würde festgelegt, daß vom Postulat des Freihandels auszugehen ist und die Länder einschränkenden Maßnahmen auflisten müssen. Nationale Regulierungen sollen des weiteren einem „Notwendigkeitstest“ unterzogen werden, um die Rahmenbedingungen für den Dienstleistungshandel möglichst liberal zu gestalten (Hoekman 2000, Mattoo 2001).

Es bleibt also festzuhalten, daß sich die Effizienz der Verhandlungen erheblich verbessern ließe, wenn man die Verhandlungskonventionen veränderte und zu multilateralen Gesprächen überginge. Auch der Wechsel von der Positivliste zur 
Negativliste, wie beim GATT gebräuchlich, würde den Liberalisierungsdruck auf die einzelnen Länder verstärken. Die Besonderheiten des Verhandlungsgegenstandes, nämlich der Dienstleistungen, werden im nächsten Kapitel erörtert.

\subsection{Internationaler (Bank-)Dienstleistungshandel}

\subsubsection{Banken als Dienstleistungsunternehmen}

\section{Dienstleistungen allgemein}

Unter dem Terminus „Dienstleistung“ werden verschiedene Inhalte zusammengefaßt. Der Begriff erfährt eine große Bedeutungsvielfalt, denn er kann nach Fetzer $(1992,4)$ Bezug nehmen auf

1. bestimmte Wirtschaftszweige (z.B. Unternehmen, die Dienstleistungen produzieren),

2. Produktionsergebnisse (die auch außerhalb von

Dienstleistungsunternehmen erstellt sein können) sowie

3. Berufe, ökonomische Funktionen bzw. Tätigkeiten.

Diese Fülle an Bedeutungen erklärt die hohe Zahl von Definitionsversuchen. ${ }^{1}$ McDonald (2001, 24) spricht in diesem Zusammenhang auch von einem „,...Versagen des volkswirtschaftlichen Dienstleistungsbegriffs".

Vielversprechend erscheint die Abgrenzung nach Kriterien, aber auch hier existieren Grenzfälle, die eine eindeutige Trennung von Gütern und Dienstleistungen erschweren. Als Eigenschaften von Dienstleistungen benennt die Literatur in der Regel:

- Immateriell,

- Unsichtbarkeit,

- Vergänglichkeit und mangelnde Lagerfähigkeit,

- Mangelnde Transportfähigkeit,

- Gleichzeitigkeit von Konsum und Produktion,

- Hoher Anteil von Humankapital, geringer Anteil von Sachkapital.

Eine umfassende Definition des Dienstleistungsbegriffs jüngeren Datums entwickelte Rück $(2000,277)$. Ihr hoher Abstraktionsgrad ist nötig, um die vielfältigen Ausprägungen von Dienstleistung zu erfassen:

1 Eine intensive Auseinandersetzung mit dem Dienstleistungsbegriff ist u.a. zu finden bei Maleri (1994), Stibora / de Vaal (1995), Fetzer (1992) sowie Ruck (2000). 
„Dienstleistungen sind Transformationsprozesse, die zu gewerblichen Zwecken an externen Faktoren (Wirtschaftseinheiten und / oder deren Verfügungsobjekten) erbracht werden und eine Veränderung der Zustandseigenschaften dieser Faktoren bewirken. "

- Die Leistung wird mit Transformation gleichgesetzt; damit ist eine Dienstleistung in ihrer Ausprägung, daß sie eine ,Bearbeitung' herbeiführt und eine Produktion darstellt, erfaßt.

- Leistung ist ein unbestimmter Oberbegriff; er umfaßt sowohl menschliche Arbeitsleistungen wie auch Maschinenleistungen. Dienstleistungen sind also Prozesse, sie umfassen aber auch das Ergebnis des Bearbeitungsprozesses.

- Nur gewerbliche Leistungen, also solche, die nach dem Gewinnprinzip erbracht werden, sind von der Definition abgedeckt. Unentgeltliche Dienste werden nicht erfaßt.

- Die Einschränkung, daß Prozesse an externen Faktoren vollzogen werden müssen, verhindert, daß innerbetriebliche, unternehmensinterne Vorleistungen, die einen Dienstleistungscharakter aufweisen, inkludiert werden.

- Nicht nur endverbraucherbezogene, sondern auch unternehmensbezogene Dienstleistungen sollen erfaßt werden; deshalb wurde die Formulierung Wirtschaftseinheiten statt Personen gewählt.

- Verfügungsobjekte sind alle wirtschaftlichen Güter, die für die Dauer des Produktionsprozesses Eigentum des Leistungsnehmers sind (real- aber auch nominalwirtschaftliche Faktoren wie Informationen, Rechte und Geld).

- Die Notwendigkeit einer (zeitlich) synchronen Integration externer Faktoren bedarf keiner gesonderten Erwähnung, sie ist Voraussetzung für eine Transformation und damit implizit (Rück 2000, 277 ff.).

Diese Begriffsdefinition verdeutlicht hinlänglich die Komplexität des Dienstleistungsbegriffs und damit auch die Schwierigkeit, Dienstleistungen innerhalb der ökonomischen Theorie bzw. der Außenhandelstheorie angemessen einzubinden. Die Analyse aller Dienstleitungen in ihrer außenwirtschaftlichen Dimension ist wenig praktikabel, weshalb diese Arbeit sich auf die Betrachtung der internationalen Bankdienstleistungen beschränkt. Damit kann auch die Arbeitsdefinition folgendermaßen konkretisiert werden.

\section{Die Bankdienstleistungen}

Bankdienstleitungen weisen spezielle Charakteristika auf und bedürfen einer eingehenden Betrachtung. Generell werden unter dem Begriff der Bankdienstleistungen alle Leistungen einer Bank subsummiert. Diese gliedern sich in die Bereiche 
Kredit (Aktiv- und Passivgeschäft), Zahlungsverkehr und sonstige Dienste (Rück 2000, 289). Diese Charakterisierung des Bankgeschäfts orientiert sich an dem in Deutschland vorherrschenden Typus der Universalbank, die verschiedene Leistungen, oftmals in Form von Leistungsbündeln, anbietet.

Die Zuordnung der Produktionsergebnisse der Banken zum Dienstleistungssektor liegt in deren überwiegender Immaterialităt sowie ihrem prozessualen Charakter begründet. Auch die durchgängige Auftragsgebundenheit und damit die Unmöglichkeit der Vorratsproduktion (bzw. die Notwendigkeit des Absatzes vor der Produktion sowie der Integration externer Faktoren), ihre Komplexität und Erklärungsbedürftigkeit rechtfertigen die Bezeichnung als Dienstleistung. Konkret handelt es sich um vier Leistungsarten, die von Banken erbracht werden:

1. Das Kreditgeschäft, ${ }^{\prime}$

2. das Handelsgeschäft (Wertpapiere, Edelmetalle, Devisen, Kredite und Rechte),

3. das Zahlungsverkehrs- oder Überweisungsgeschäft und

4. das Aufbewahrungsgeschäft (physische Aufbewahrung von Wertgegenständen wie auch abstrakte Aufbewahrung von Wertpapieren in Depots).

\subsubsection{Die bank- bzw. einzelwirtschaftliche Sichtweise}

Die Bankbetriebswirtschaft nimmt einen etwas anderen Blickwinkel ein. Demnach produzieren Banken nicht nur die o.g. absatzfähigen Dienstleistungsprodukte. Diese Betrachtungsweise wird ergänzt durch die Berücksichtigung der Interbanken- und Eigenleistungen.

Unter den primären Leistungen werden erfaßt (Eilenberger 1987, 114 ff.):

- Kreditleistungen:

Kreditgewährung (originäre Kreditleistung) und Kreditvermittlung (derivative Kreditleistung),

- Anlageleistungen:

Übernahme von Geld- und Kapitalnutzung unterschiedlicher Fristigkeiten durch Bankbetriebe seitens der Kundschaft (originäre Anlageleistungen) und Vermittlung derartiger Nutzungsmöglichkeiten bei anderen Bankbetrieben sowie Unternehmungen an die Bankkundschaft (derivative Anlageleistungen) gegen Entgelt,

- Zahlungsverkehrsleistungen:

Überbrückung der räumlichen Distanz zwischen Vorrätigkeit und Bedarf

Nach Ruck $(2000,290)$ handelt es sich hierbei um „...die entgeltliche Überlassung von Nutzungsrechten an Nominalgütern auf Zeit. ". 
des immateriellen Nominalgutes Geld aufgrund der Kundenbedürfnisse gegen Entgelt und

- Sonstige Bankmarktleistungen:

Entgeltliche und unentgeltliche Dienstleistungen, die den anderen Haupttypen nicht eindeutig zugeordnet werden können wie Kundenberatung und -betreuung, Depotleistungen und Garantieleistungen, Edelmetallhandelsleistungen für Kunden, Beratung bei spezifischen Finanzierungsproblemen (Innovationsfinanzierung, Kapitalerhöhungen), Vermittlung von Beteiligungskapital (Venture Capital), Fusionsberatung, Branchenanalysen, Immobilienvermittlung, treuhänderische Vermögensverwaltung und sonstige Kundendienstleistungen.

$\mathrm{Zu}$ den sekundären Leistungen zählen:

- Interbankenleistungen:

Produktion von Dienstleistungen für den Interbankenmarkt, die aus Liquiditäts- und Rentabilitätsüberlegungen erfolgt wie Übernahme von Geldund Kapitalanlagen einer anderen Bank aus dem In- und Ausland, Kreditleistungen an andere Banken, Interbanken-Zahlungsverkehrsleistungen und

- Eigenleistungen:

Erstellung von Eigenleistungen für eigene Zwecke im Bereich des Handels von Devisen, Sorten, Edelmetallen und Effekten, ebenso Beteiligungserwerb und -verkauf sowie Zahlungsverkehrsleistungen für eigene Zwecke.

Auch in diesem Sinne sind Banken generell als Dienstleistungsbetriebe zu kennzeichnen.

Zusammengefaßt erfüllt eine Bank also drei Funktionen:

- Angebot und Schaffung von Finanzierungsmöglichkeiten für andere Betriebswirtschaften,

- Angebot und Durchführung von Geldanlagemöglichkeiten und

- Angebot und Durchführung des Zahlungsverkehrs.

Durch die vielfältigen Verflechtungen zwischen Banken und Versicherungen im Finanzbereich und auch aus Gründen der Risikostreuung werden häufig Bündel von Dienstleistungen angeboten, die aus einer Kombination von Bank- und Versicherungsleistungen bestehen (z.B. ein Kreditvertrag, der durch eine Lebensversicherung besichert wird). In solchen Fällen ist eine eindeutige Zuordnung nicht möglich. Dieses Problem macht auch eine klare statistische Erfassung schwierig. Entsprechend werden nur Zahlen für Finanzdienste in ihrer Gesamtheit ermittelt, nicht aber dezidierte Aufschlüsselungen nach einzelnen Positionen vorgenommen. Damit werden quantitative Bestimmungsversuche des Protektionsgrades erheblich 
erschwert. Preis- oder mengenbasierte Methoden können kaum verwendet werden, weil das Zahlenmaterial hierfür nicht ausreichend detailliert aufgeschlüsselt ist. Vielversprechender ist ein Ansatz, der die Wettbewerbsbedingungen für den Bankensektor zu erfassen sucht. Dieses Konzept repräsentiert die Zinsmarge, die in dieser Arbeit als Indikator verwendet wird. ${ }^{1}$

Der Bankensektor weist wirtschaftstheoretisch einige Besonderheiten auf, so z.B. die hohe Regulierungsdichte ${ }^{2}$, die in seiner volkswirtschaftlichen Funktion als Finanzintermediär begründet liegt. Den volkswirtschaftlichen Aufgaben der Banken widmet sich das nächste Kapitel.

\subsubsection{Die volkswirtschaftliche Funktion von Banken}

Banken bergen neben ihrem betriebswirtschaftlichen Charakter als Unternehmungen, die der Gewinnerzielung dienen, auch eine zentrale volkswirtschaftliche Funktion. $^{3}$ Als finanzwirtschaftlicher Intermediär sind sie zum einen die Vermittler zwischen den ersparnisbildenden Haushalten und den investierenden Unternehmen, zum anderen sind sie auch unerläßlich für die Transmission der zentralbankgelenkten Geldpolitik und für die Aufrechterhaltung der finanzpolitischen Stabilität eines Landes (Mishkin 2001, 33). Levine (1996) verweist auf folgende fünf Grundfunktionen von Banken: Sie erleichtern den Austausch von Gütern und Diensten (1), erleichtern das Risikomanagement (2), mobilisieren finanzielle Ressourcen (3), sammeln und verwerten Informationen, evaluieren Unternehmen und alloziieren Kapital (4) und üben Unternehmenskontrolle aus (5). Diese Funktionen sind von zentraler Bedeutung für eine marktwirtschaftlich organisierte Wirtschaft, und die mit der Bereitstellung von Finanzdiensten verbundenen Externalitäten sind substantiell (Poveda / Droege 1997, 603 f.).

Durch ihre Funktion als Intermediär zwischen Kapitalgebern und -nehmern reduzieren Banken die Transaktionskosten der Beteiligten. Von zentraler Bedeutung ist die Transformation kurzfristiger Depositen (Einlagen) in langfristige Kredite. Diese Fristentransformation ist unerläßlich für die langfristigen Investitionspläne der Unternehmen, denen aber oftmals ein zeitlich kürzerer Anlagehorizont der Hauhalte entgegensteht. Erst durch die Bündelung kleinerer Einlagen, die sich in ihrer Zusammensetzung mit der Zeit ändern können, ist es möglich, Kredite mit langen Laufzeiten zu vergeben und längerfristige Investitionen $\mathrm{zu}$ finanzieren.

Banken helfen, die Informationsasymmetrien zwischen Kreditgebern und -nehmern zu verringern. Dadurch kann der Bankensektor adverse Selektion vor Durchführung der Transaktion verhindern oder zumindest einschränken, da die

\footnotetext{
Vgl. hierzu ausfuhrlicher Kapitel 3 Zinsmargen als Indikator des Protektionsgrades.

Vgl. zu expliziten Einzelheiten Moschel (1990), insbes. Kapitel 2 Regulations as Obstacles to International Free Trade in Banking Services, $71 \mathrm{ff}$.

3 Fur eine Übersicht aktueller Fragestellungen vgl. Bhattacharya / Thakor (1993).
} 
Gefahr besteht, daß sich verstärkt schlechte Risiken um einen Kredit bemühen. Eine wahrscheinliche Folge wäre die Kreditverknappung, die sich auch auf die guten Risiken im Markt negativ auswirken würde. Banken helfen auch moral hazard-Probleme nach der Transaktion zu beseitigen, denn ihre Kontrollen stellen sicher, daß die erteilten Finanzaktiva nicht zweckentfremdet werden (Mishkin 2001, 34 ff.). Ein leistungsfähiges, verläßliches Bankensystem erleichtert somit den ökonomischen Agenten die Umsetzung ihrer Ersparnis- und Investitionspläne zu geringen Kosten und unterstützt damit die effiziente Allokation des Faktors Kapital.

Aus volkswirtschaftlicher Sicht ist ebenfalls die Rolle der Banken für die Transmission der Geldpolitik zu erwähnen. Diese enge Verflechtung zwischen der Zentralbank und den Kreditinstituten existiert aufgrund ihrer Bedeutung für den Geldschöpfungsprozeß. Die Zentralbank kann den geschäftspolitischen Entscheidungsraum der Banken durch direkte Auflagen (Mindestreserveanforderungen, Refinanzierungskontingente) beschränken. Damit übt sie einen indirekten Einfluß auf die Preispolitik der Banken aus, da sie deren Kostensituation und damit ihre Rentabilitätsüberlegungen beeinflußt (Reszat 1988, 389 f.). Diese Aspekte finden in der weiteren Analyse nur Berücksichtigung, wenn sie die (betriebswirtschaftlichen) Kosten der Banken beeinflussen. Auf eine Analyse der Bedeutung der Banken für die Geldpolitik wird verzichtet.

Der Bankensektor ist einer der am stärksten regulierten Wirtschaftsbereiche. ${ }^{1}$ Ziele der Regulierung sind aus mikroökonomischer Sicht der Schutz und die Sicherheit der Kunden und zwar sowohl der Einleger wie auch der Kreditnehmer. Damit steht die Sicherung der individuellen Bank und ihrer Kunden im Vordergrund. Auf makroökonomischer Ebene stellt die Verhinderung von Bankenkrisen das Ziel der Regulierung dar; die Stabilität des kompletten Banken- und Finanzsystems soll gewährleistet werden (Baltensperger 1989/90, 2). Konkurse von Bankinstituten münden nicht selten in schwerwiegenden Erschütterungen des gesamten Finanzsystems (bank runs) (Reszat 1988, 390 und 398). Denn Banken und Finanzmärkte zeichnen sich nach Minsky (1978) durch eine dem Geldsystem inhärente Instabilität aus, die sog. financial instability hypothesis. Diese Instabi-

1 Als Hauptgrunde fur die Bankenregulierung benennen Barth, Caprio und Levine (2002, 4 f.):

(1) Interessenkonflikte der Banken durch die Vielfaltigkeit ihrer Aufgaben (Sicherheiten der eigenen Kreditkunden an schlecht informierte Anleger zu verkaufen),

(2) moral hazard fordert die Risikobereitschaft (und je weiter das Betătigungsfeld der Banken, um so großer die Møglichkeiten hierfur),

(3) komplexe Banken sind schwieriger zu uberwachen als spezialisierte,

(4) große Banken konnen politisch und ðkonomisch so mächtig werden, daß sie kaum noch zu disziplinieren sind,

(5) große Finanzkonglomerate kőnnen den Wettbewerb und die Effizienz des Systems verringern.

Ihre Schlußfolgerung lautet, daß die Funktionsweise des Bankwesens verbessert werden kann, wenn Bankaktivitaten staatlich begrenzt werden. 
lität beruht nicht auf irrationalen Entscheidungen oder aufgrund von Marktunvollkommenheiten, sondern ist die Folge einer Kombination von psychologischen, soziologischen und politischen Faktoren, die das Marktgeschehen prägen. Mit zunehmender Dauer einer konjunkturellen Boom-Phase wandeln sich ursprünglich robuste Finanzierungsstrukturen im Zeitablauf in risikoreiche und störungsanfällige. Die professionellen Anleger werden im Verlauf dieser Aufschwungphase immer wagemutiger, da alle Projekte, die ein kalkulierbares Risiko tragen bereits finanziert sind. So bleibt nur die Übernahme von Projekten, die sich durch ein höheres Risiko auszeichnen, das aber nicht immer ausreichend abgesichert werden kann. Daraus entsteht eine spekulative Blase, deren Platzen nach Minsky unausweichlich ist (Schnyder 2002, 62 ff.). Regulierungen dienen im optimalen Fall der Erhöhung der Finanzstabilität, der Verhinderung der Entwicklung einer solchen Blase und der Abmilderung aller mit ihrem Platzen verbundenen Folgen.

Für die weitere Analyse ist also zu beachten, daß unter dem Begriff der Banken primär Institutionen verstanden werden, die als Intermediäre zwischen Anbietern und Nachfragern von Geldmitteln auftreten. Banken werden damit in erster Linie als Geldsammelstellen betrachtet, die finanzielle Mittel für Investoren bereitstellen. Diese Dienstleistung steht im Mittelpunkt ihrer Tätigkeit. Weitere von ihnen erbrachte Dienstleistungen (z.B. Zahlungsverkehr, Aufbewahrung von Aktien und Wertpapieren etc.) ergänzen diesen Hauptzweck ihrer Geschäftstätigkeit.

\subsubsection{Dienstleistungsverkehr}

Der internationale Handel zeichnet sich durch den Verkauf von Gütern an die Einwohner anderer Länder aus. In der Regel erfolgt die Produktion im Inland und anschließend der Transport der Waren über die Landesgrenzen hinweg. Aufgrund des physischen Charakters von Sachgütern kann durch den Grenzübertritt die Handelsaktivität im internationalen Rahmen statistisch erfaßt werden. Der Dienstleistungshandel weist diesbezüglich Besonderheiten auf, denn ein Grenzübertritt kann durch die überwiegende Immaterialität der Dienste in den seltensten Fällen beobachtet werden.

\subsubsection{Formen des Handels}

Aufgrund ihrer Spezifikationen ${ }^{1}$ erfordert die Erstellung von sowie der Handel mit Dienstleistungen zumeist die direkte Beteiligung des Konsumenten am Produktionsort, so daß im Vergleich zum Sachgüterhandel neue Handelsoptionen

1 Vgl. hierzu die Definition von Dienstleistungen in Kapitel 2.2.1 Banken als Dienstleistungsunternehmen sowie Walter (1985, insbes. $12 \mathrm{ff})$. 
gefunden werden müssen. Die Leistungserstellung kann in vier Erbringungsarten (den sog. 4 Modi) erfolgen: ${ }^{1}$

\section{Grenzüberschreitende Transaktion in das Bestimmungsland hinein} (Separated services, Mode 1):

Die Dienstleistungen überschreiten, analog zu Sachgütern, die Grenze. Dienstleistungen dieser Kategorie müssen lagerfähig, ohne Mitwirkung des Abnehmers zu produzieren und transportabel sein (Fetzer 1992, 20; Feketekuty 1988, 16 f.). Dies trifft insbesondere für die in Sachgütern verkörperten Dienste zu (Software, Bücher etc.). Diese Form des direkten Dienstleistungshandels umfaßt hinsichtlich der Banken die Vergabe von Großkrediten, Exportversicherungen und -finanzierungen, denn sie erfordern keine individuelle Anpassung, sondern können pauschal angeboten und auf postalischem oder elektronischem Wege zugestellt werden.

\section{Konsum im Herstellungsland durch Grenzübertritt der Konsumenten (Provider located services, Mode 2):}

Der Nachfrager wird für die Diensterstellung vor Ort benötigt und muß den Produktionsort aufsuchen. Typische Beispiele hierfür sind die Bereiche Tourismus, Bildung, medizinische Dienste sowie Großreparaturen (bspw. in ausländischen Werften).

\section{Transaktion durch den Grenzübertritt des Anbieters in das Bestim- mungsland (Demander located services, Mode 3):}

Diese Dienste müssen direkt am Ort der Abnahme angeboten werden, es handelt sich überwiegend um arbeitsintensive Konsumentendienstleistungen wie z.B. Bauleistungen, Tanz- oder Theaterauffuhrungen. Der Aufenthalt des Anbieters kann zeitlich begrenzt sein oder permanent in Form einer Agentur zur Unterstützung der Dienstleistungsexporte erfolgen. ${ }^{2}$

Nachfrager und Anbieter überschreiten eine Grenze (Footloose oder non-separated services, Mode 4):

Die Dienstleistung wird in einem Drittland angeboten und erstellt, diese Situation stellt eine Kombination der Modi 2) und 3) dar.

Der dritte Erbringungsmodus ist für Banken besonders relevant, da sie oftmals in Form eigener Niederlassungen im Ausland tätig sind (Poveda / Droege 1997,

1 Sampson und Snape (1985) waren die ersten, die eine Einteilung des Dienstleistungshandels gemăß diesen Kategorien vornahmen, die von der Weltbank und der UNCTAD ubernommen wurden und anschließend auch Eingang in das GATS fanden, vgl. hierzu auch Stibora / de Vaal $(1995,23)$, Krancke (1994, 10 ff.) und Fetzer (1992, 20 ff.)

2 Moglich sind die Grundung einer dauerhaften Produktionsstătte im Ausland (Direktinvestition), Franchising oder die Lizenzproduktion im Zielland. 
609). Sie bieten beratungsintensive Produkte an (Konsumentendienstleistungen), die ihre physische Anwesenheit erfordern wie auch pauschalierte Dienste, die ohne Beratung (Produzentendienste) und über alternative Vertriebswege (Postweg, Internet) angeboten werden können. In der Zahlungsbilanz werden die Bankenaktivitäten entweder als Handel oder aber - durch die Direktinvestition als inländische Produktion eingestuft (Fetzer 1992, 24 sowie Chen / Schembri 2002, 220). Diese unterschiedliche Erfassung erschwert die Bestimmung des tatsächlichen Handelvolumens. In Analogie zum Güterhandel gilt für die statistische Erfassung nur der Austausch gemäß der ersten Erbringungsart (Modus 1) als Handel. Die räumliche Nähe als Bedingung für die Dienstleistungserstellung kann durch die Nutzung technischer Hilfsmittel erreicht werden (Internet, Telefon etc.). Die anderen drei Kategorien des Handels bedürfen der Verlagerung von Produktionsfaktoren bzw. des Konsumenten über Staatsgrenzen. Die herkömmlichen Statistiken müßten erweitert werden um die Betrachtung, daß die Produktion von Firmen in ausländischem Besitz als Importe zu gelten habe. Zur näherungsweisen Erfassung dieser schlecht beobachtbaren Größen werden die ausländischen Direktinvestitionen (Foreign Direct Investment, FDI) verwendet. Sie dienen als Schätzwert zur Quantifizierung des anders nicht beobachtbaren Dienstleistungshandels (Stibora / de Vaal 1995, 23 sowie 38).

\subsubsection{Wichtige Aspekte internationaler Dienstleistungstransaktionen}

\section{Die Bedeutung von Direktinvestitionen}

Dienstleistungen bedürfen zumeist der Präsenz des Anbieters am Erbringungsort. Entsprechend erfolgen Direktinvestitionen im Ausland (Filiale, Tochterunternehmen, Fusionen oder Akquisition) oder es wird eine temporäre Aufenthaltsform in geeigneter Form (Joint Venture, kurzfristiger Auslandsaufenthalt) gewählt. Direktinvestitionen weisen erhebliche Vorteile auf, denn die Grenzüberschreitung von Produktionsfaktoren ist in der Regel mit erheblichen Transaktionskosten verbunden, die sich bei temporär begrenzten Aufenthalten kaum amortisieren würden. Existiert die Perspektive auf eine längerfristige Betätigung auf dem Auslandsmarkt, so stellt eine Verlagerung der Produktion ins Zielland oft die kostengünstigere Alternative dar. Außerdem können auf diese Weise strategisch bedeutende Märkte erschlossen werden.

Durch ihre Immaterialität liefern viele Dienstleistungen nicht ausreichend Informationen über ihre Eigenschaften; sie sind ausgesprochen beratungsintensiv. In dieser asymmetrischen Informationslage kennt nur der Anbieter die qualitativen Eigenschaften der Dienstleistung. Ein Großteil der Dienstleistungen weist - ebenfalls entscheidungserschwerend - einen hohen Grad an Produktdifferenzierung auf; die Standardisierung ist oftmals schwierig, wenn nicht unmöglich. Dieser hohe Beratungsbedarf erzeugt Unsicherheit beim Käufer, die beide Seiten durch eine langjährige und erfolgreiche Beratungs- und Verkaufsbeziehung abzubauen 
suchen. Für die Pflege langjähriger Beziehungen erweist sich die dauerhafte geographische Nähe zum Kunden als förderlich (Sapir 1987, 50; o.V., 2000, 35).

Die Dienstleistungsproduktion unterliegt oftmals einer starken staatlichen Regulierung, welche die Niederlassungspflicht vorschreibt, um auf dem Zielmarkt agieren zu dürfen. In diesem Fall sind Direktinvestitionen (insbesondere bei Finanzdienstleistungen) der einzige Weg des Marktzugangs. Betrachtet man staatliche Behinderungen der Dienstleistungsexporte ausländischer Konzerne als Externalitäten, so können diese am effizientesten durch Direktinvestitionen internalisiert werden (Rugman 1987, 651 ff.).

Welcher Vertriebsweg für eine Bank, die ihre Dienstleistungen im Ausland anbieten will, vorteilhaft ist, hängt von zahlreichen Faktoren $a b .{ }^{1}$ Sie kann den direkten Vertrieb über FDI wählen, als Exporteur ohne eigene Niederlassung agieren oder beide Wege parallel beschreiten. Von zentraler Bedeutung für diese Frage ist die Entscheidung, ob FDI und Handel als Substitute oder Komplementäre betrachtet werden. Erbringen Banken im Ausland Dienstleistungen, ohne dort über eigene Niederlassungen zu verfügen, so sind FDI und internationaler Dienstleistungsverkehr Substitute. Die Empirie belegt aber auch, daß mehr Dienstleistungen in Länder exportiert werden, in denen inländische Banken über Tochtergesellschaften oder Filialen verfügen. Dies läßt wiederum auf eine komplementäre Beziehung zwischen FDI und Bankdienstleistungen schließen (Buch / Lipponer 2004, 10 ff.; 24). Je höher die Investitionen sind, die im Ausland getätigt werden, um so mehr Dienstleistungen werden dorthin exportiert. Allerdings ist zu beachten, daß der Interbankendienstleistungsverkehr zwischen Mutter- und Tochtergesellschaft die Daten wahrscheinlich verfälscht.

Banken bevorzugen den Marktzugang durch FDI bei kultureller, sprachlicher und räumlicher Nähe zum Zielland. Unter diesen Bedingungen sind expansive Strategien in der Regel erfolgreich. Auch die Größe des Auslandsmarktes (gemessen durch das absolute Bruttoinlandsprodukt wie auch das Pro-Kopf-Einkommen), ein hohes bilaterales Handelsvolumen, geringes Risiko und freier Kapitalverkehr beeinflussen die Entscheidung positiv. Liegen hingegen Kapitalverkehrsbeschränkungen vor, eine sehr ausgeprägte oder aber sehr schwache Regulierung des ausländischen Bankenmarktes, so wird der Export der Dienste gegenüber der risikobehafteten Alternative der Investition eigenen Kapitals bevorzugt.

Ein Marktzutritt durch eine Fusion mit inländischen Bankinstituten bietet einige Vorteile. Marktzugangsbarrieren für Dienstleistungsexporte können auf diesem Wege überwunden werden. In dem Entscheidungsprozeß der Banken, sich gegen den Dienstleistungsexport und für eine Fusion zu entscheiden, stellt der hohe Grad an Marktnähe ein wichtiges Kriterium dar. Durch den Erwerb einer Geschäftsbank vor Ort werden lokale Marktkenntnisse (Know-how der Mitarbeiter) wie

Vgl. insbesondere Tschøgel (2003), Buch / DeLong (2003) sowie Buch / Lipponer (2004). 
auch der bereits existierende Kundenstamm übernommen. Damit ist die Bank gegenüber einem nur Dienstleistungen exportierenden Institut bevorteilt. Auch eine Kaufentscheidung wird durch die Faktoren Größe des Auslandsmarktes, Ausmaß der Handelsbeziehungen, Eintrittsbeschränkungen wie auch Grad der Produktdifferenzierung beeinflußt.

Die Entscheidung für einen adäquaten Fusionspartner ist abhängig von der (längerfristigen) Geschäftsstrategie der einzelnen Bank. Ein Argument für die Fusion von Geschäftsbanken untereinander ist das Motiv der economies of scale. Soll hingegen die Geschäftstätigkeit auf neue Bereiche erweitert werden, um economies of scope zu realisieren, so werden Geschäftsbankenfusionen mit anderen Finanzinstitutionen angestrebt (Buch / DeLong 2003, 232 ff.).

Während der 1990er Jahre dominierte zunächst weniger eine internationale Fusionswelle als die heimische Konsolidierung des Bankensektors (Fusionen mit und Akquisitionen von inländischen Banken), erst danach erfolgte eine gemäßigte, grenzüberschreitende Merger-Welle. Die Second Banking Directive der EU hat die Dienstleistungserbringung innerhalb der Mitgliedstaaten sehr erleichtert. Bankenfusionen innerhalb der EU sind deshalb weniger bedeutend und seltener als Fusionen mit Nicht-EU-Banken, die den Vorteil der Erschließung eines anderweitig nicht erreichbaren Auslandsmarktes beinhalten.

Eine Studie von Cybo-Ottone und Murgia (2000) ermittelte, daß inländische Fusionen die besten Erfolgsaussichten aufweisen; sie dienen gleichzeitig der Konsolidierung des Bankenmarktes. Statistisch betrachtet glücken auch reine Geschäftsbankenfusionen sowie Fusionen von Geschäftsbanken mit Versicherungsunternehmen. Grenzüberschreitende Bankenfusionen hingegen sind relativ selten erfolgreich, so daß der Handel mit Bankdienstleistungen weiterhin ein wichtiger Vertriebsweg bleibt.

\section{Die Bedeutung moderner Technologien}

Der Einsatz moderner Technologien im Kommunikations- und Informationsbereich sowie bei Transporten hat erheblich zur Senkung der Transaktionskosten beigetragen. Damit ist der Handel vieler Dienstleistungen zu günstigen Konditionen möglich. Zudem sind Dienstleistungen exportierbar, die noch vor kurzem als unhandelbar galten. Technische Hilfsmittel wie Telefon, Fax oder Internet ermöglichen es, mit einem relativ geringen Aufwand Informationen und Angebote weltweit einzuholen sowie Aufträge an ausländische Dienstleister zu erteilen. Durch moderne Telematik-Methoden lassen sich gerade die informationsbasierten Dienste sehr gut handeln, da auch große Informationsmengen günstig, schnell und

1 Die Second Banking Coordination Directive wurde 1989 beschlossen und um die Investment Services Directive im Jahre 1993 ergänzt. Vgl. hierzu u.a. http://www.riskglossary.com/link/european_financial_regulation.htm. 
zuverlässig über weite Strecken transportiert werden können. Durch die stetige Weiterentwicklung technischer Verfahren werden immer mehr ehemals nicht handelbare Dienste in handelbare transformiert (Fetzer 1992, 33).

Auch die Mobilität der Menschen hat sich zunehmend erhöht (Hochgeschwindigkeitszüge, Flugzeuge), so daß selbst große Distanzen in kurzer Zeit zurückgelegt werden können. Damit sind Anreisen zum Erfüllungsort seitens des Abnehmers oder des Anbieters von Dienstleistungen ökonomisch rentabel geworden.

\section{Die Interdependenz zwischen Waren- und Dienstleistungsverkehr}

Die Verfügbarkeit grundlegender Dienstleistungen wie effizienter und verläßlicher Banken-, Versicherungs- und Telekommunikationssysteme ist unerläßlich für die Produktionslandschaft eines Landes. Ist ein Land nicht in der Lage, ein vertrauenswürdiges und funktionierendes Bankensystem zu etablieren, so müssen diese Dienste aus dem Ausland importiert werden. Eine unzureichende Bankenstruktur vermindert die Entwicklungs- und Wachstumsaussichten eines Landes, da Investitionen unterbleiben. Dies wirkt sich in der Folge negativ auf die produzierenden Sektoren, die inländische Versorgung wie auch den Export aus (Feketekuty 1988, 24 und 35).

Generell können Dienstleistungen und Güter in einem substitutiven Verhältnis, einem komplementären Verhältnis oder einem unabhängigen Verhältnis zueinander stehen (Stern / Hoekman 1987, 41). Unter einem substitutiven Verhältnis wird der warenersetzende Handel verstanden, wie er bspw. durch das FranchisingSystem repräsentiert wird. In einem komplementären Verhältnis bedingen Warenund Dienstleistungshandel einander, bei einem unabhängigen Verhältnis stehen sie in keinerlei Bezug zueinander wie z.B. bei Lebensversicherungen, Tourismus. In der Regel ist die Komplementarität zwischen Dienstleistungen und Waren um so ausgeprägter, je technologisch hochwertiger die Waren sind. Sie zeichnen sich dann zumeist durch eine hohe Beratungsintensität aus. Um den Kundenwünschen gerecht werden zu können, werden Güter und Dienstleistungen zu maßgeschneiderten Paketen geschnürt. Derartige Geschäftsmodelle sind zum Teil in der Computerbranche üblich. Einige Anbieter zeichnen sich durch die kundenadäquate Zusammenstellung von Hard- und Software. Sie setzen sich damit deutlich von konkurrierenden Unternehmen ab, die stark standardisierte Rechner anbieten. ${ }^{1}$

1 Als Beispiel sei auf das Geschaftsmodell des Unternehmens Dell ${ }^{\mathrm{TM}}$ verwiesen. Die Computer werden aus gewünschten Hardware-Bausteinen erst auf Bestellung hin und nicht bereits vorrätig gebaut. Ebenso können die gewunschte Betriebssoftware und die Anwendungsprogramme zur Installation ausgewăhlt werden. Der Vorteil dieser Vorgehensweise: Vormontierte Rechner bergen die Gefahr der schnellen Veralterung einzelner Komponenten. Da Dell ${ }^{\mathrm{TM}}$ aber erst auf Bestellung hin tătig wird und den Kunden eine Vielzahl von Alternativkomponenten anbietet, kőnnen Rechner produziert werden, die exakt auf die Bedurfnisse des Kunden abgestimmt sind. Abgesehen von den Dienstleistungen, die im Rechner inkorporiert sind 
Dienstleister folgen auch ihren warenproduzierenden Kunden auf die Auslandsmärkte. Wird eine kritische Masse an warenproduzierenden Niederlassungen erreicht, so lassen sich Dienstleister für Buchhaltung, Controlling und auch Banken durch Direktinvestitionen im Ausland nieder, um langjährige Geschäftsbeziehungen vor Ort aufrechterhalten zu können (Fetzer 1992, 38). Aber auch der umgekehrte Weg wird praktiziert. Die Warenproduzenten folgen Dienstleistern ins Ausland, weil sie sich auf eine qualitativ gleichbleibende Beratung verlassen können. Diese Entwicklung ist neben der Filmindustrie und der Werbung insbesondere bei Banken zu beobachten (Sapir 1982, 79 f.; Rada 1987, 140 ff.).

\section{Probleme der Erfassung von Dienstleistungsströmen}

Dienstleistungen weisen einen hohen Mangel an statistischer Erfaßbarkeit auf, da ihr Grenzübertritt oftmals nicht nachvollziehbar ist. Bankdienstleistungen werden als Bestandteil der Financial Services erfaßt. Die meisten Statistiken können die Volumina für Versicherungen und Bankleistungen aber nicht separat ausweisen. Durch die vielfältigen Verflechtungen zwischen Banken und Versicherungen im Finanzbereich und auch aus Gründen der Risikostreuung werden häufig Bündel von Dienstleistungen angeboten, die aus einer Kombination derselben bestehen. In solchen Fällen ist eine eindeutige Zuordnung nicht möglich. Entsprechend werden die Zahlen für Finanzdienste in ihrer Gesamtheit aufbereitet, nicht aber dezidierte Aufschlüsselungen nach einzelnen Positionen. Herkömmliche Methoden zur Ermittlung des Protektionsgrades wie preis- oder mengenbasierte Meßverfahren, die für den Güterhandel entwickelt wurden, lassen sich für die Analyse von Dienstleistungsströmen nicht verwenden. Der Ansatzpunkt für protektionistisches Verhalten ist deshalb in der Regulierung der Niederlassungsrechte zu sehen (IMF / World Bank 2001, 39).

\subsubsection{Hemmnisse im Dienstleistungshandel}

Protektionistisches Verhalten nationaler Regierungen findet seinen Ausdruck in wirtschaftspolitischen Maßnahmen, die der Benachteiligung ausländischer Unternehmen gegenüber ihren inländischen Konkurrenten dienen.

„Von Protektionismus ist bei jener Kerngruppe von Hemmnissen zu sprechen, die gezielt den Zutritt ausländischer Marktteilnehmer erschweren (Verweigerung der Gleichbehandlung mit Inländern) bzw.

(System- und Anwendungsprogramme) stellt die Maßanfertigung an sich eine Dienstleistung dar, die mit einer Sachgutproduktion untrennbar verknupft ist. Vgl. Dell ${ }^{\mathrm{TM}}$-Homepage vom 03. April 2006: http://wwwl.euro.dell.com/content/topics/topic.aspx/emea/topics/ how_to shop/ purchase_assistance?c $=$ de\&cs $=$ dedhs $1 \& \mathrm{l}=\mathrm{de} \& \mathrm{~s}=\mathrm{dhs} \& \sim \mathrm{ck}=\mathrm{mn}$. 


\section{die Wettbewerbssituation von Inländern künstlich verbessern." (Schultz 1985, 182).}

Die hohe Regulierungsdichte ist ein Charakteristikum vieler Dienstleistungssektoren. Dies erschwert die Unterscheidung in „notwendige“ und „protektionistische" Regulierungen. Als protektionistisch ist eine Regulierungsbestimmung einzuordnen, wenn das Regulierungsziel mit einer alternativen Maßnahme erreicht werden könnte, die weniger oder gar nicht diskriminierend gegenüber ausländischen Anbietern wirken würde (Feketekuty 1998, 113).

Die Beseitigung solcher Hemmnisse im Dienstleistungsbereich erfordert also weitreichende Eingriffe in die nationalen Regulierungssysteme. Im Warenhandel können qualitative Anforderungen direkt an das Produkt geknüpft werden, die Zollerhebung ist beim Grenzübertritt möglich. Bei Dienstleistungen ist die Produktionsphase vom Produkt selbst oft nicht zu trennen. Die Regulierung setzt deshalb beim dienstleistenden Produzenten an und zwar durch die Gestaltung der Bedingungen hinsichtlich Markteintritt und Geschäftstätigkeit. Damit stehen als protektionistische Instrumente nur die nichttarifären Hemmnisse (NTB) zur Verfügung. Das Dilemma der NTB's besteht darin, daß sie negativ definiert werden, nämlich als alle Handelsmaßnahmen, die nicht Zölle sind. Hierunter werden nicht nur Handelsbeschränkung verstanden, sondern auch Handelsförderungen wie Exportsubventionen. Letztlich zählen auch alle politischen Maßnahmen, die in irgendeiner Weise die gehandelten Mengen und Preise beeinflussen, dazu (Deardorff / Stern 1997, 4). Damit umfassen sie administrative Prozeduren (Lizenzierung, Genehmigungsverfahren für Importe, Antidumping-Untersuchungen etc.) wie auch Marktstrukturen, die durch unterschiedliche Regulierungen von Monopolen, ökonomischen Aktivitäten, öffentlichen Unternehmen etc. entstanden sind. Auch institutionelle Faktoren wie die politischen, sozialen und kulturellen Institutionen, staatliche Interventionen oder mangelnde Bestreitbarkeit der Märkte können sich als Handelshemmnisse erweisen (Krancke 1999, 14 f.).

Ein weiteres Problem liegt in der mangelnden Transparenz der Handelshemmnisse. Sie verbergen sich in mannigfachen Regulierungsbestimmungen und deren Kombinationen. Auch die alltägliche Verwaltungspraxis oder mangelnde Schulung des administrativen Personals kann in (versteckter) Diskriminierung münden. Empirisch belegt ist die Tendenz bei Regierungsbeamten, eine gewisse Affinität zu dem von ihnen verwalteten Wirtschaftsbereich zu entwickeln und ihn (mehr oder minder bewußt) vor ausländischer Konkurrenz schützen zu wollen (Feketekuty 1988, $141 \mathrm{f}$.).

Die Hemmnisse im Dienstleistungshandel nehmen vielfältige Formen an und lassen sich in fünf Hauptgruppen unterscheiden (Fetzer 1992, 65): ${ }^{1}$

1 Fur das Bankenwesen liegen sektorspezifische Untersuchungen existierender Handelshemmnisse bspw. von Dale (1986), OECD (1984a), Walter (1981) und Gavin (1985) vor. 
1. Maßnahmen, die die Möglichkeit eines ausländischen Unternehmens schmälern, seine Dienste anzubieten und zu verkaufen,

2. Maßnahmen, die auf eine Verweigerung des Marktzutritts hinauslaufen (quantitativ und / oder qualitativ),

3. Maßnahmen, die den finanziellen Geschäftsverkehr behindern,

4. Maßnahmen der öffentlichen Hand, die diskriminierende oder entmutigende Wirkungen auf ausländische Unternehmen haben können, sowie

5. Maßnahmen, die den Zugang zu produktionsnotwendigen Vorleistungen behindern, auf die ein Unternehmen im Ausland angewiesen ist.

Einen Einblick in die Ausprägungsformen der Hemmnisse gibt die folgende Tabelle im Detail. 
a Tab. 2-1: Handelshemmnisse im Dienstleistungsbereich

\begin{tabular}{|c|c|c|c|c|}
\hline $\begin{array}{l}\text { Verweigerung des Markt- } \\
\text { zutritts (quantitativ und } / \\
\text { oder qualitativ) }\end{array}$ & \begin{tabular}{|c|} 
Einschrånkungen im \\
Angebot bzw. Verkauf der \\
Dienste
\end{tabular} & $\begin{array}{l}\text { Diskriminierung des } \\
\text { Finanzverkehrs }\end{array}$ & $\begin{array}{c}\text { Diskriminierung durch die } \\
\text { offentliche Hand }\end{array}$ & $\begin{array}{c}\text { Zugangbehinderung zu } \\
\text { produktionsnotwendigen } \\
\text { Vorleistungen }\end{array}$ \\
\hline $\begin{array}{l}\text { Verbot von Dienst- } \\
\text { leistungsimporten }\end{array}$ & Verbot von Marketing & $\begin{array}{l}\text { Verweigerung von } \\
\text { Devisen fur importierte } \\
\text { Dienstleistungen }\end{array}$ & $\begin{array}{l}\text { Nationale Vorbehalte } \\
\text { bei Beschaffungsmaß- } \\
\text { nahmen der offentlichen } \\
\text { Hand }\end{array}$ & $\begin{array}{l}\text { Behinderung beim } \\
\text { Import von Ersatzteilen }\end{array}$ \\
\hline $\begin{array}{l}\text { Verbot der Nieder- } \\
\text { lassungsgrüundung }\end{array}$ & $\begin{array}{l}\text { Kontingente bezüglich } \\
\text { des Umsatzes oder der } \\
\text { Marktanteile }\end{array}$ & $\begin{array}{l}\text { Diskriminierende Devi- } \\
\text { senkontrolle, Verzoge- } \\
\text { rung der finanztechni- } \\
\text { schen Abwicklung von } \\
\text { Auslandstransfers (Ge- } \\
\text { winne, Abgaben etc.) }\end{array}$ & $\begin{array}{l}\text { Diskriminierende } \\
\text { juristische Behandlung }\end{array}$ & $\begin{array}{l}\text { Behinderung beim Im- } \\
\text { port wichtiger Aus- } \\
\text { rustungsgegenstände furr } \\
\text { den Betrieb eines } \\
\text { Dienstleisters im Inland }\end{array}$ \\
\hline $\begin{array}{l}\text { Diskriminierende oder } \\
\text { schikanठ̋se Vergabe- } \\
\text { praktiken bei Importli- } \\
\text { zenzen oder Genehmi- } \\
\text { gungen fur inländische } \\
\text { Niederlassungen, Erlaß } \\
\text { von Einzelvorschriften } \\
\text { fur bereits zugelassene } \\
\text { Filialen }\end{array}$ & $\begin{array}{l}\text { Beschränkung der Ver- } \\
\text { tragsfreiheit (inländische } \\
\text { Transporte dürfen nicht } \\
\text { von ausländischen } \\
\text { Transporteuren } \\
\text { ubernommen werden } \\
\text { (Kabotageverbot)) }\end{array}$ & $\begin{array}{l}\text { Obergrenzen fur } \\
\text { Devisenzahlungen für } \\
\text { die Überlassung aus- } \\
\text { ländischer Technologien } \\
\text { (Gebühren, Provisionen } \\
\text { etc.) }\end{array}$ & $\begin{array}{l}\text { Regierungseigene } \\
\text { Dienstleistungsbetriebe } \\
\text { unterbieten auf den in- } \\
\text { und / oder ausländischen } \\
\text { Mărkten }\end{array}$ & $\begin{array}{l}\text { Beschränkung der Ein- } \\
\text { kaufsquellen furr aus- } \\
\text { ländische Firmen } \\
\text { (diskriminierende } \\
\text { Praktiken staatlicher } \\
\text { inländischer } \\
\text { Monopolanbieter) }\end{array}$ \\
\hline $\begin{array}{l}\text { Beschrankung des Aus- } \\
\text { landsreiseverkehrs (we- } \\
\text { niger potentielle Kăufer } \\
\text { ausländischer Dienst- } \\
\text { leistungen gelangen zum } \\
\text { Angebotsort) }\end{array}$ & $\begin{array}{l}\text { Nationaler Vorbehalt bei } \\
\text { der Kustenschiffahrt }\end{array}$ & $\begin{array}{l}\text { Zwang zur Beteiligung } \\
\text { Einheimischer am } \\
\text { Grundkapital bereits } \\
\text { existierender Filialen im } \\
\text { Inland („Localization“) }\end{array}$ & $\begin{array}{l}\text { Zusătzliche formale } \\
\text { Erfordernisse bei } \\
\text { ausländischen } \\
\text { Unternehmen }\end{array}$ & $\begin{array}{l}\text { Unzulänglicher Schutz } \\
\text { von Copyrights und } \\
\text { Markenzeichen }\end{array}$ \\
\hline
\end{tabular}




\begin{tabular}{|c|c|c|c|c|}
\hline $\begin{array}{l}\text { Verweigerung des Markt- } \\
\text { zutritts (quantitativ und / } \\
\text { oder qualitativ) }\end{array}$ & \begin{tabular}{|c|} 
Einschrănkungen im \\
Angebot bzw. Verkauf der \\
Dienste
\end{tabular} & $\begin{array}{l}\text { Diskriminierung des } \\
\text { Finanzverkehrs }\end{array}$ & $\begin{array}{c}\text { Diskriminierung durch die } \\
\text { offentliche Hand }\end{array}$ & $\begin{array}{c}\text { Zugangbehinderung zu } \\
\text { produktionsnotwendigen } \\
\text { Vorleistungen }\end{array}$ \\
\hline & $\begin{array}{l}\text { Gesetzliche Grenzen bei } \\
\text { der Preisgestailtung, z.B. } \\
\text { Mindestpreise, die } \\
\text { ausländische Konkur- } \\
\text { renz nicht unterbieten } \\
\text { darf (Versicherungen) }\end{array}$ & $\begin{array}{l}\text { Diskriminierende } \\
\text { Zollerhebung und - } \\
\text { abfertigung bei in Waren } \\
\text { inkorporierten Diensten }\end{array}$ & $\begin{array}{l}\text { Einsatz technischer } \\
\text { Regeln und Standards } \\
\text { mit dem Ziel, } \\
\text { ausländische } \\
\text { Unternehmen zu } \\
\text { benachteiligen }\end{array}$ & $\begin{array}{l}\text { Diskriminierende Be- } \\
\text { schaftigungskontingente } \\
\text { oder Verbot der } \\
\text { Beschäftigung Ange- } \\
\text { höriger bestimmter } \\
\text { Rassen oder Religionen }\end{array}$ \\
\hline & $\begin{array}{l}\text { Beeinträchtigung der mit } \\
\text { dem Angebot von } \\
\text { Dienstleistungen ver- } \\
\text { bundenen Aktivităten } \\
\text { (Werbung, Kom- } \\
\text { munikation) }\end{array}$ & $\begin{array}{l}\text { Diskriminierende } \\
\text { Besteuerung }\end{array}$ & Zensur & $\begin{array}{l}\text { Zwang zur Preisgabe } \\
\text { vertraulicher } \\
\text { Geschaftsinterna }\end{array}$ \\
\hline & $\begin{array}{l}\text { Begrenzung der Aus- } \\
\text { wahl an Dienstlei- } \\
\text { stungsunternehmen furr } \\
\text { inlăndische Kunden }\end{array}$ & \multirow{3}{*}{$\begin{array}{l}\text { Verweigerung (staatl.) } \\
\text { Vergünstigungen bei } \\
\text { Firmen mit ausländi- } \\
\text { scher Beteiligung (Ex- } \\
\text { portkredite, Subventio- } \\
\text { nen, gespaltener Wech- } \\
\text { selkurs bei der Zentral- } \\
\text { bank für Devisenankauf) }\end{array}$} & $\begin{array}{l}\text { Geringe Transparenz } \\
\text { administrativer Abläufe }\end{array}$ & $\begin{array}{l}\text { Administrative Schwie- } \\
\text { rigkeiten bei der Ertei- } \\
\text { lung von Visa und Auf- } \\
\text { enthaltsgenehmigungen }\end{array}$ \\
\hline & \multirow{2}{*}{$\begin{array}{l}\text { Festsetzung eines An- } \\
\text { teils von Dienst- } \\
\text { leistungen, der von in- } \\
\text { ländischen Firmen er- } \\
\text { bracht werden muB }\end{array}$} & & \multirow[t]{2}{*}{$\begin{array}{l}\text { Subventionen fur inlăn- } \\
\text { dische Betriebe }\end{array}$} & $\begin{array}{l}\text { Beschrankung der Be- } \\
\text { wegungsfreiheit aus- } \\
\text { ländischen Personals }\end{array}$ \\
\hline & & & & $\begin{array}{l}\text { Nichtanerkennung } \\
\text { ausländischer Zeugnisse } \\
\text { und Diplome }\end{array}$ \\
\hline
\end{tabular}




\section{Handelshemmnisse im Bankensektor}

Behinderungen des internationalen Dienstleistungsverkehrs im Bankensektor können auf verschiedenen Ebenen erfolgen. Die Besonderheit des GATS liegt darin, daß es praktisch unbeschränkte Kombinationen von Hemmnissen und deren graduelle Abstufung ermöglicht. So kann die staatliche Kontrolle der Finanzströme oder des Bankensektors die Bandbreite von geringer bis hin zur vollständigen Kontrolle ausschöpfen. Auch die vollständige Verwehrung des Marktzugangs ist praktikabel.

Da sich die im Members Schedule aufgeführten Konditionen ausschließlich gegen ausländische Banken richten, treten die protektionistischen Absichten klar zutage. Diese Bestimmungen sind offensichtliche Protektion, die sich in dem Verbot einzelner Geschäftstätigkeiten (z.B. des Aufbringens von Depositen), der Beschränkung der Kreditvergabe (hinsichtlich der Höhe und/oder der Art der Kredite oder der Branchen) oder Vorschriften bezüglich der Etablierung auf dem inländischen Markt (z.B. Zwangsfusion mit einer heimischen Bank) äußern.

Weitaus schwieriger zu ermitteln ist die versteckte Protektion, die sich in weiteren Regulierungen und administrativen Vorschriften verbergen kann. Diese werden nur dann als Protektion betrachtet, wenn sie diskriminierend eingesetzt werden. So sind bspw. die Behörden eines Landes in der Lage, entscheidende Nachteile für ausländische Unternehmen zu schaffen. Diese Behinderungen gehen eindeutig auf die staatliche Ebene zurück.

Behinderungen im Marktzugang können aber auch von privater Seite erfolgen. So können Preisstrategien (Kartelle) verfolgt werden, um ausländische Konkurrenten abzuwehren (strategische Marktzugangsbeschränkungen), oder aber auch strukturelle Marktzugangsbeschränkungen bestehen z.B. in Form von Betriebsgrößen- und Kostenvorteilen. Diese Formen der Wettbewerbsbehinderung sind nur dann als Protektion zu betrachten, wenn dieses Verhalten sich gezielt gegen ausländische Konkurrenten richtet und von staatlicher Seite unterstuitzt bzw. nicht unterbunden wird.

Zur Messung der Auswirkungen von protektionistischen Bestimmungen bietet sich die Verwendung des Indikators Zinsmarge an. Ihm widmet sich das nächste Kapitel. Da sich eine empirische Auswertung anschließt, wird auch Bezug auf das vorliegende Datenmaterial genommen und eine qualitative Auswertung vorgenommen. Zinsmargen reflektieren die existenten Marktbedingungen in ihrer vollständigen Komplexität, sie sind damit Indikator in zweierlei Dimension: Sie spiegeln sowohl die betriebswirtschaftliche Effizienz der Banken wie auch die gesamtwirtschaftlichen Rahmenbedingungen wider. 


\subsection{Zwischenfazit}

Das zweite Kapitel schlägt insgesamt einen großen thematischen Bogen und streift mehrere Punkte, die nun noch einmal kurz hervorgehoben werden.

Die allgemeine Erläuterung der Protektion im Welthandel und die detaillierte Diskussion des GATS als Instrument des Protektionsabbaus dient der Verdeutlichung der Rahmenbedingungen, innerhalb derer sich diese Arbeit bewegt. Die komplexe Struktur des GATS ist den Besonderheiten des Handelsgutes „Dienstleistung" geschuldet, die insbesondere in seiner Immaterialität begründet sind. Die Charakteristika des GATS erfordern eine unnötig umständliche Arbeitsweise der Delegationen und erklären die erhebliche Dauer der Handelsrunden zur Reduzierung der Handelshemmnisse. Bedeutender aber ist der Umstand, daß die Bestimmungen des GATS die Handelshemmnisse prägen, die für Dienstleistungen im Allgemeinen und Bankendienste im Besonderen gelten. Regulierungen müssen nicht primär aus protektionistischen Gründen erhoben werden, sie können auch der Aufrechterhaltung der Stabilität des Banken- und Finanzsystems geschuldet sein. Die Unterscheidung zwischen den nötigen, im Sinne der erforderlichen, und den unnötigen, da protektionistischen Regulierungen zu treffen, ist eine Herausforderung.

Als Instrument der Protektionsmessung wird das Konzept der Zinsmarge verwendet, das im folgenden Kapitel detailliert dargestellt wird. Informationen zu den nationalen Finanz- und Bankensystemen werden anschließend als Variablen verwendet, die auf die Zinsmarge regressiert werden. Argumentativ werden zunächst die Variablen erläutert, die tatsächlich über Protektionspotential verfügen. Diese Analyse findet in Kapitel 4.1.3 Variablengruppen und ihre Protektionspotentiale statt. Anschließend werden die Regressionen durchgeführt, um die Variablen herauszufiltern, die Determinanten der Zinsmargen sind. Entsprechend zeichnet sich in der Folge ab, welche Regulierungen tatsächlich die Zinsmargen determinieren und vor allem welche Determinante über Protektionspotential verfügt. Erst nach Ermittlung der protektionistisch wirkenden Variablen können wirtschaftspolitische Empfehlungen formuliert werden, die einer effektiven Liberalisierung der Märkte für Bankendienstleistungen dienlich sind. 


\section{Zinsmargen als Indikator des Protektionsgrades}

\subsection{Das Konzept der Zinsmarge}

Die klassische Analyse von Zinsen beschäftigt sich entweder mit deren Struktur, also dem Verhältnis zwischen kurz- und langfristigen Zinsen ${ }^{1}$, oder mit der Bestimmung des Gleichgewichtszinses auf Kapitalmärkten. ${ }^{2}$ In der makroökonomischen Analyse treffen auf dem Kapitalmarkt Anbieter und Nachfrager von finanziellen Mitteln aufeinander. Die Spar- und Investitionspläne der Marktteilnehmer werden durch den sich bildenden Gleichgewichtszinssatz in Übereinstimmung miteinander gebracht.

Tatsächlich übernehmen Banken als Intermediäre diese Koordinationsaufgaben. Sie operieren nicht zu einem einheitlichen Zins am Markt, sondern unterscheiden zwischen Depositen- und Kreditzinsen. Diese Differenz wird als Zinsmarge bezeichnet. Zinsmargen versetzen Banken in die Lage, die Fristen-, Risiko- und Losgrößentransformationen zwischen Einlagen und Krediten vorzunehmen, denn sie decken die eigenen Betriebskosten und generieren Gewinne. Aus Sicht der Banken sind Kredite und Depositen hinsichtlich ihrer Eintrittswahrscheinlichkeit, ihrer Höhe und Kosten ungewiß. Hinzu kommt das Risiko eines (Total-)Verlustes bei Krediten. ${ }^{3}$ Banken kompensieren diese Informationsmängel und Risiken durch die adäquate Setzung der Zinsmargenhöhe.

Zinsmargen dienen der Kostendeckung, sie werden deshalb auch als „, banker's mark-up" bezeichnet. ${ }^{4}$ Zur Berechnung werden die Nettozinsgewinne der Banken ins Verhältnis zu den zinstragenden Vermögenswerten gesetzt. Die operationale

1 Zu den geläufigsten Erklärungsansătzen gehören die Erwartungs-, Marktsegmentations- oder Liquiditătsprämientheorie.

2 Diesbezliglich existieren viele Ansătze bspw. die klassische Theorie (zinsgesteuerte Investitionsnachfrage der Unternehmen), Liquiditătstheorie (Liquiditătskasse der Haushalte), Portfoliotheorie (Zinsabhängigkeit der Geldhaltung, da Opportunitătskosten in Form entgangener Wertpapierrenditen Berlicksichtigung finden) oder die Loanable-funds-Theorie (Zins wird auf dem Kreditmarkt bestimmt, Kreditangebot durch Ersparnisse der Haushalte und Geldschøpfungen der Banken, Kreditnachfrage durch Investitionsvolumen und Horten).

3 Eine detaillierte Analyse der Zusammenhänge zwischen Zinsrisiken und Nettozinsmargen ist bei English (2002) zu finden.

4 Banken stehen grundsătzlich zwei Einnahmequellen zur Verfugung: Zinseinnahmen und Gebuhren. Beide Formen dienen der Deckung anfallender Ausgaben. Gebuhren, die fur die Erbringung einzelner Dienstleistungen durch die Banken entrichtet werden mussen, werden nicht weiter berücksichtigt. Zur Verwendung der durchschnittlichen Bankengebuhren eines Landes fur eine Regression ist das vorliegende Zahlenmaterial leider nicht ausreichend. Es gibt aber Hinweise darauf, daß diese Einnahmenquelle zur Kostendeckung an Bedeutung gewinnt. Steigender Konkurrenzdruck und eine hohe Transparenz der Zinsniveaus verschiedener Banken senken die Einnahmen. Banken fokussieren sich deshalb verstărkt auf die Einnahmengenerierung durch Gebuhren. 
Definition der Nettozinsmarge (NIM = Net Interest Margin) lautet (in Anlehnung an die Reserve Bank of Australia 1994, 1):

$N I M=\frac{\sum \text { Zinseinkommen netto }}{\sum \text { Wert der zinstragenden Assets }}=\frac{\sum \text { Sollzinsen }-\sum \text { Habenzinsen }}{\sum \text { Wert der zinstragenden Assets }}$.

Zinsmargen repräsentieren verschiedene Aspekte:

a) Sie dienen als ein Effizienzmaß der Vermittlung der Ressource „Kapital“.

b) Sie repräsentieren die Rentabilität der Banken, denn sie müssen ausreichend hoch gewählt werden, um die Betriebskosten zu decken.

c) Sie decken das Ausfallrisiko der Kredite, d.h. daß an ihnen die bankoder landesspezifische Mischung von Vermögenswerten und Verbindlichkeiten ablesbar ist.

Hohe Zinsmargen werden in der Regel mit weniger gut entwickelten Märkten assoziiert. Diese weisen in der Regel hohe (Bank-)Betriebskosten, Marktzugangsbarrieren und einen niedrigen Wettbewerbsgrad auf (Kannan et.al. 2001, 337). Sollte der Markt bereits liberalisiert sein und immer noch hohe Zinsmargen aufweisen, so indiziert dies einen Mangel an Wettbewerb durch Monopolmacht der ansässigen Banken. Relativ niedrige Zinsmargen sind ein Signal für einen funktionierenden Wettbewerb und effiziente Strukturen. Ausnehmend niedrige Zinsen bergen aber auch die Gefahr, daß die Gewinnspannen der Banken sehr reduziert sind und sie u.U. ihr Eigenkapital beanspruchen müssen. Dies kann die Krisenanfälligkeit einer Bank, u.U. sogar des gesamten Systems erhöhen.

Zinsen erfüllen die Funktion des Preises auf dem Kapitalmarkt und steuern somit das Angebot von und die Nachfrage nach Finanzmitteln. Preise sind sich stetig in Bewegung befindliche Größen. Die Ursachen von Preisvariationen liegen angebotsseitig in den Veränderungen der Produktionskosten oder der Gewinnspannen. Diesen Entwicklungen kann als Ursache ein protektionistischer Eingriff seitens der Regierung vorweggegangen sein. Maßnahmen, die zwingend den Einsatz von inländischen Rohstoffen oder Mitarbeitern vorschreiben, werden in der Regel kostenerhöhend wirken. Eine Erhöhung von Gewinnen verhindert im Regelfall der Wettbewerb. Betreibt die Regierung jedoch die vollständige Abschottung des Bankenmarktes gegenüber ausländischen Kreditinstituten, sind die Folgen ein sinkender Wettbewerbsdruck und steigende Marktmacht derselben. Die Banken werden diesen Umstand tendenziell zur Erhöhung ihrer Gewinne nutzen.

Auch die institutionellen und ökonomischen Rahmenbedingungen einer Volkswirtschaft, innerhalb derer die Banken agieren, finden ihre Reflexion in den Zinshöhen, denn sie bestimmen die Höhe des Aufschlags, des „banker's mark-up“, der auf die von der Zentralbank vorgegebenen Leitzinsen erhoben wird. Die Zinshöhe ist somit ein Indikator für bankenwirtschaftliche (In-)Effizienz, vorherrschende 
Marktbedingungen, Regulierung wie auch Protektion. In diesem Sinne spiegeln hohe Zinsmargen einen Mangel an Wettbewerb wider. Diesem Zustand liegen entweder eine mono- bzw. oligopolistische Marktstruktur (die marktbeherrschende Stellung einer oder weniger Banken) zugrunde und/oder die Marktabschottung gegenüber ausländischen Konkurrenten.

Mangelnder Wettbewerb resultiert in der ineffizienten Bereitstellung der Dienstleistung „Kapitalvermittlung“. Einzelwirtschaftlich betrachtet setzen die Banken am Markt überhöhte Preise gegenüber ihren Kunden durch, womit hohe Produzentenrenten abgeschöpft werden. In der Folge richten die Banken Überkapazitäten und eine überbordende Administration ein, da der Kostensenkungsdruck durch effizientere Konkurrenten fehlt. Die gesamtwirtschaftliche Folge ist die Fehlallokation des Produktionsfaktors Kapital. Verzerrte Preise, d.h. in diesem Fall überhöhte Zinsmargen, führen zu falschen Dispositionen der Marktteilnehmer. Investitionen unterbleiben, da die Kreditzinsen zu hoch angesetzt werden. Auch die Depositen bleiben aufgrund einer zu geringen Verzinsung hinter dem optimalen Niveau zurück. Damit büßt die Volkswirtschaft Wachstumschancen ein, der Produktionsfaktor Kapital wird nicht optimal alloziiert.

Eine der ersten empirischen Untersuchungen der Determinanten von Zinsmargen erbrachten Ho und Saunders (1981). ${ }^{1}$ Zur Aufrechterhaltung der Solvenz bilden Banken Reserven in Höhe der Ausfallprämien ihrer Kredite. Als den Anteil einer Zinsmarge, der diese Reservenbildung erfaßt, identifizierten Ho und Saunders den "pure spread".

Die Höhe des pure spread wird von vier Einflußgrößen determiniert:

a) Risikoaversion des Bankmanagements,

b) Umfang und Höhe der von der Bank getätigten Transaktionen,

c) Struktur des Bankenmarktes und

d) Zinsvolatilität.

Der pure spread allein erklärt jedoch nicht vollständig die Zinsmarge. Nach Saunders und Schumacher (2000) sind Kapital- und Liquiditätsbestimmungen sowie die operativen Kosten des Bankbetriebs von positivem und signifikantem Einfluß auf die Zinsmargen. Regelungen, die der Aufrechterhaltung der Stabilität des Finanzsystems dienen wie Mindestreserve- und Liquiditätsbestimmungen, werden als ",prudential regulation" bezeichnet.

Diese Ansätze weiterführend schätzten Kalirajan et.al. (2000) den Einfluß wettbewerbsbeschränkender Regulierungen (non-prudential regulation) auf die Zins-

1 Eine aussagekrăftige Kommentierung der verschiedenen Forschungsansătze der Analyse der Zinsmargen liefern Chen / Schembri (2002). 
margen. Für die Messung des Restriktionsgrades, also des praktizierten Ausmaßes an Protektion, griffen sie auf das Konzept des Trade Restrictiveness Index zurück. Das Protektionsmaß des Trade Restrictiveness Index entwickelte ein australisches Forscherteam ${ }^{1}$. Es wertete neben den GATS-Protokollen jede weitere verfügbare Quelle aus, um alle Informationen über die Restriktionen zusammenzutragen. Die ermittelten Regulierungen werden anschließend gemäß ihres restriktiven Wirkungsgrades mit Punkten versehen und zu Gruppen zusammengestellt. Die Gruppen werden dann noch einmal gewichtet. Die Verteilung der Punkte und Gewichte auf die einzelnen Restriktionen wird hinsichtlich des verursachten ökonomischen Effizienzverlustes getätigt: Je höher die Verluste an ökonomischer Effizienz, die durch eine Restriktion entstehen, um so höher ihr Gewicht. Damit handelt es sich um ein sehr subjektives Gewichtungsschema. Anschließend werden zwei Indizes gebildet, der ausländische und der inländische Index (,foreign and domestic index'). Hierbei umfaßt der ausländische Index (a) Restriktionen, die nur für ausländische Unternehmen gelten, der inländische Index ${ }^{2}$ (b) hingegen erfaßt die Restriktionen, die gleichmäßig auf alle Anbieter angewendet werden. Der Vorteil dieses Verfahrens liegt in der Möglichkeit, die Diskriminierung ausländischer Konkurrenten in der Differenz (b) - (a) ermitteln zu können. Damit ist eine Verletzung des von der WTO geforderten Prinzips des, national treatment', d.h. der Gleichbehandlung aller Unternehmen ohne Beachtung ihres Herkunftslandes $\mathrm{zu}$ belegen. Der Trade Restrictiveness Index wandelt also qualitative Aussagen über den Offenheitsgrad eines Landes in eine quantitative Größe um, um diesen als Regressor in Schätzungen verwenden zu können. McGuire und Schuele haben dieses Verfahren in einer Studie (2001) für den Sektor der Bankendienstleistungen angewendet. In ihren Trade Restrictiveness Index gehen folgende Regelungen ein:

- Vergabe von Banklizenzen (Gewicht 0,2),

- Direktinvestition in inländische Banken (Gewicht 0,2),

- Joint Ventures (Gewicht 0,1),

- Aufenthaltsrecht für ausländische Mitarbeiter (Gewicht 0,02),

- Aufbringen von Finanzmitteln/Einlagen (Gewicht 0,15),

- Beschränkung der Kreditvergabe (Gewicht 0,15),

- Weitere Geschäftsfelder: Versicherungen und Wertpapiere (Gewicht 0,10 ),

- Filialgründungen (Gewicht 0,05),

I Es handelt sich hierbei um ein gemeinsames Forschungsprojekt der Australia's Productivity Commission, University of Adelaide, und der Australian National University.

2 Der domestc index ist damit auch ein Indikator fur eventuelle Beschränkungen des Marktzugangs. 
- Zusammensetzung der Geschäftsführung (Gewicht 0,02),

- Temporärer Aufenthalt von Mitarbeitern / Spezialisten für max. 90 Tage (Gewicht 0,01).

Damit soll ein möglichst realistisches Bild der existierenden Regelungen entworfen werden. Die konkrete Bewertung der qualitativen Informationen stellt folgende Tabelle 3-1 dar. 'Kalirajan et.al. kamen in ihrer Schätzung unter Verwendung des Trade Restrictiveness Index zu dem Ergebnis, daß der Offenheitsgrad eines Landes von signifikantem Einfluß auf die Zinsmargen ist: Je weniger Handelsbeschränkungen ein Land aufweist, um so niedriger seine Zinsmarge. Zusammenfassend verdeutlicht folgende Graphik die Zusammensetzung der Zinsmarge.

\section{Abb. 3-1: Determinanten der Zinsmargen}

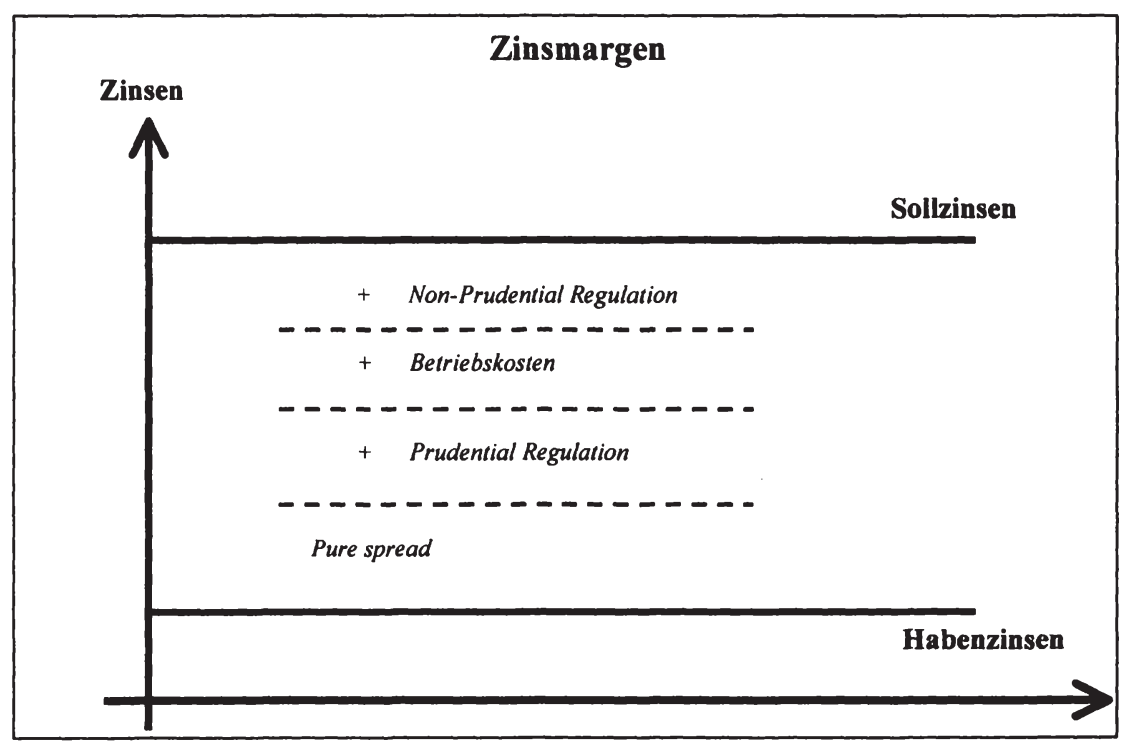

Eigene Darstellung.

In der linken Spalte befindet sich die Punktwertung der einzelnen Restriktion. 


\section{Tab. 3-1: Bewertungsschema des Trade Restrictiveness Index:}

Restriktionen im Bankensektor

\begin{tabular}{|c|c|}
\hline \multicolumn{2}{|r|}{ Licensing of banks (Weight 0,20 ) } \\
\hline 1,00 & Issue no new bank licences \\
\hline 0,75 & Issues up to three new banking licences with only prudential requirements \\
\hline 0,50 & Issues up to six new banking licences with only prudential requirements \\
\hline 0,25 & Issues up to ten new banking licences with only prudential requirements \\
\hline 0,00 & Issues new banking licences with only prudential requirements \\
\hline \multicolumn{2}{|r|}{ Direct Investment (Weight $\mathbf{0 , 2 0}$ ) } \\
\hline \multicolumn{2}{|r|}{$\begin{array}{l}\text { The score is inversely proportional to the maximum equity participation permitted in an } \\
\text { existing domestic bank. Equity participation to a maximum of } 75 \text { percent of a bank } \\
\text { would receive a score of } 0,25\end{array}$} \\
\hline \multicolumn{2}{|r|}{ Joint-Venture Arrangement (Weight 0,10 ) } \\
\hline 1,0 & $\begin{array}{l}\text { Issues no new bank licences and no entry is allowed through a joint venture with a } \\
\text { domestic bank }\end{array}$ \\
\hline 0,50 & Bank entry is only through a joint venture with a domestic bank \\
\hline 0,00 & No requirements for a bank to enter through a joint venture with a domestic bank \\
\hline \multicolumn{2}{|r|}{ Permanent movement of people (Weight 0,02 ) } \\
\hline 1,00 & No entry of executives, senior managers and/or specialists \\
\hline 0,80 & Executives, senior managers and/or specialists can stay up to one year \\
\hline 0,60 & Executives, senior managers and/or specialists can stay up to two year \\
\hline 0,40 & Executives, senior managers and/or specialists can stay up to three year \\
\hline 0,20 & Executives, senior managers and/or specialists can stay up to four year \\
\hline 0,00 & Executives, senior managers and/or specialists can stay up to five year \\
\hline \multicolumn{2}{|r|}{ Raising funds by banks (Weight 0,15 ) } \\
\hline 1,00 & Banks are not permitted to raise funds in domestic market \\
\hline 0,75 & Banks are not permitted to raise funds in domestic capital market \\
\hline 0,50 & Banks are restricted in accepting deposits from the public \\
\hline 0,00 & Banks can raise funds from any source with only prudential requirements \\
\hline
\end{tabular}




\section{Tab. 3-1: Bewertungsschema des Trade Restrictiveness Index (Forts.)}

\begin{tabular}{|c|c|}
\hline \multicolumn{2}{|r|}{ Lending funds by banks (Weight 0,15 ) } \\
\hline 1,00 & Banks are not permitted to lend to domestic clients \\
\hline 0,75 & Banks are restricted to a specific lending size or lending to government projects \\
\hline 0,50 & $\begin{array}{l}\text { Banks are restricted in providing certain services such as credit cards, leasing and } \\
\text { consumer finance }\end{array}$ \\
\hline 0,25 & Banks are directed to lend on housing and small business \\
\hline 0,00 & Banks can lend on any source with only prudential restrictions \\
\hline \multicolumn{2}{|r|}{ Other business of banks: insurance and security services (Weight 0,10 ) } \\
\hline 1,00 & Banks can only provide banking services \\
\hline 0,50 & $\begin{array}{l}\text { Banks can provide banking services plus one other line of business: insurance or security } \\
\text { service }\end{array}$ \\
\hline 0,00 & No restrictions on conducting other line of business \\
\hline \multicolumn{2}{|r|}{ Expanding the number of banking outlets (Weight 0,05 ) } \\
\hline 1,00 & One outlet with no new outlets permitted \\
\hline 0,75 & Number of outlets is limited in number and location \\
\hline 0,25 & Expansion of outlets subject to non-prudential regulatory approval \\
\hline 0,00 & No restrictions on banks expanding operations \\
\hline \multicolumn{2}{|r|}{ Composition of the board of director (Weight 0,02 ) } \\
\hline \multicolumn{2}{|r|}{$\begin{array}{l}\text { The score is inversely proportionate to the percentage of the board that can comprise } \\
\text { foreigners. For example, a score of } 0,80 \text { is allocated where } 20 \text { percent of the board of } \\
\text { directors can comprise foreigners }\end{array}$} \\
\hline \multicolumn{2}{|r|}{ Temporary movement of executives, senior managers and/or specialist } \\
\hline 1,00 & No temporary entry \\
\hline 0,75 & Temporary entry up to 30 days... \\
\hline 0,5 & Temporary entry up to 60 days... \\
\hline 0,25 & Temporary entry up to 90 days... \\
\hline
\end{tabular}

Quelle: Mc Guire / Schuele (2001, 204 f.).

Die Banken wählen die Zinshöhen derart, daß das Kreditverlustrisiko (pure spread) sowie ihre eigenen Betriebskosten gedeckt sind. Hinzu kommen die Kosten, die durch Einhaltung der Mindestreserve und Liquiditätsbestimmungen entstehen (prudential regulation) sowie Aufschläge, die durch Protektion entstehen (non-prudential regulation). Der Aufschlag aufgrund von Protektion kann kostenerhöhend wirken, bspw. wenn nur inländisches Personal zu einem hohen Tariflohn eingestellt werden darf. Oder aber die Banken nutzen ihre Marktmacht mangels Konkurrenz zur Erhöhung der Gewinnspannen. Je größer das Ausmaß der Protektion, um so stärker werden die Sollzinsen erhöht und um so höher sind 
die Zinsmargen. Derart verzerrte Preise führen dazu, daß der Markt „falsche“ Signale erhält, an denen die Wirtschaftssubjekte ihr Verhalten ausrichten; in der Folge kommt es zu einer Fehlallokation des Produktionsfaktors Kapital.

Die Darstellung der Protektion im Bereich der Dienstleistungen erfolgte zunächst nur durch die reine Aufzählung der Maßnahmen, gegebenenfalls noch über die Bildung eines Index.' Bei dieser Verfahrensweise werden jedoch Informationen durch subjektive Gewichtungen und die Berechnung von Mittelwerten verfälscht. Deshalb werden Indizes in der jüngeren empirischen Forschung nicht mehr verwendet, sondern statt dessen die ökonometrischen Schätzmethoden verfeinert (z.B. Angbazo 1997, Drakos 2002, für Griechenland).

Kennzahlen können nur relative Aussagen über den vorliegenden Protektionsgrad in verschiedenen Ländern treffen, wohingegen eine Schätzung ohne den Rückgriff auf Indizes unverfälschte Aussagen über Zusammenhänge und den Wirkungsgrad der Variablen erlaubt. Um aber die tatsächlichen Auswirkungen auf den Kapitalmarkt abzubilden, sollte ein Indikator Anwendung finden, der folgende Eigenschaften aufweist: Er muß auf den Märkten gebildet werden, direkt mit der Intermediärfunktion von Banken verknüpft sein und eine quantitative Einschätzung der ökonomischen Folgen erlauben. Diese Anforderungen erfüllt der Indikator Zinsmarge, der in dieser Arbeit zur Anwendung kommt.

Zunächst wird aber anhand des folgenden Modells erläutert, wie protektionistische Regulierungen von Banken in ihrer Zinssetzung berücksichtigt werden.

\subsection{Das erweiterte Dealer-Modell}

Der theoretischen Analyse von Zinsmargen liegt das Augmented Dealership Model of Interest Spreads zugrunde. ${ }^{2}$ Es erklärt die Ermittlung der optimalen Zinshöhe durch die Banken in einer Situation, die von Unsicherheit geprägt ist. Die repräsentative Bank wird als risikoaverser Vermittler von Finanzkapital modelliert, der unter Berücksichtigung gesetzlicher Rahmenbedingungen und seiner eigenen Betriebskostenstruktur die Höhe der Depositen- und Kreditzinsen festsetzt.

Risikoaversion ist aufgrund von zwei Argumentationen unerläßlich:

Es gab zahlreiche Vorschläge zur Gestaltung von Indizes; empfohlen seien Hoekman (1995), Mattoo (1998, ,liberalization index'), das Forschungsprojekt der Australian Productivity Commission (2001, trade restrictiveness indices'), Hardin und Holmes (1997), Claessens und Glaessner (1998) sowie McGuire (1998).

2 Die Modelldarstellung erfolgt primăr in Anlehnung an Zarruk (1989). Ergănzend wurden Kannan, Narain und Ghosh (2001), McShane und Sharpe (1985), Allen (1988) sowie Angbazo (1997) hinzugezogen. 
a) Nur die Risikoaversion begründet die Existenz und das Fortbestehen von Zinsmargen. Der Liquiditätsbedarf und die -bereitstellung sind unsicher in Höhe, Eintrittsdatum und Dauer. Nachfrager wie auch Anbieter liquider Mittel bevorzugen Banken als Intermediäre statt der Direktfinanzierung (über Aktienmärkte o.ä.) aufgrund der geringeren Transaktionskosten. Die Bank als Vermittler (Dealer) betätigt sich also in einem Arbitragegeschäft, dessen Ausmaß nur von dem Grad der eigenen Risikoaversion begrenzt wird. Ohne Risikoaversion der Banken würden durch Arbitrage sämtliche Margen (bis auf die Transaktionskosten) eliminiert werden.

b) Die Annahme der Risikoaversion bewirkt, daß Banken eine endliche Größe aufweisen, da es sonst keine Begrenzung ihres Engagements in Arbitragegeschäften geben würde. Die Risikoaversion bedingt die Existenz von risikolosen Anlagemöglichkeiten am Geldmarkt (Angbazo, 1997, 58).

Traditionelle Erklärungen der Risikoaversion von Banken führen als weitere Gründe die begrenzten Diversifizierungsmöglichkeiten des Humankapitals der Manager, die unzulängliche Diversifizierung der Bankeigner, Anreizprobleme wie moral hazard und adverse selection und die Kosten, die durch den Konkurs einer einzelnen Bank oder des kompletten Systems entstehen, an.

\subsubsection{Die Annahmen des Modells}

Die Besonderheit des erweiterten Dealer-Modells liegt in der Berücksichtigung des betriebswirtschaftlichen Kalküls, das sich hinter den Zinssätzen verbirgt. Die Bereitstellungskosten der Bankdienstleistungen (administrative Kosten der Kredite und Depositen) sind für das Verhalten der Banken von großer Bedeutung, da sie - wie jedes andere privatwirtschaftliche Unternehmen - eine Maximierung ihres Gewinns anstreben.

Die Bank steht zu Beginn einer Periode vor der Entscheidung, wie hoch die Kredit- und Depositenzinsen gewählt werden sollen. Während der Laufzeit dieser Periode finden keine weiteren Zinsänderungen statt. ${ }^{1}$ Es stehen zwei Kreditformen zur Verfügung: die Gewährung von Krediten an Kunden $(L)$ und die eigene Kreditaufnahme auf dem inländischen Kapitalmarkt $(B)$. Auf der Passivseite verzeichnet die Bank Depositen (D), Anleihen auf dem inländischen Kapitalmarkt sowie Eigenkapital $(E)$.

Damit lautet die Bilanzgleichung der Bank:

(1) $L+B=D^{*}+E$.

Eine Periode dauert bis zur năchsten Zinsănderung an. 
$D^{*}$ entspricht der Summe an ex ante Depositen, d.h. den zu Beginn der Entscheidungsperiode vorliegenden Einlagen. Das Eigenkapital ist für die Dauer der Ent-

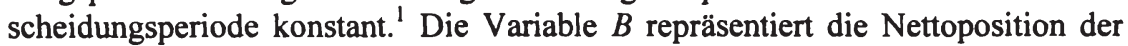
Bank auf dem inländischen Kapitalmarkt. Sei $B>0$ : Die Bank ist ein NettoKreditgeber, sie verfügt über mehr Depositen als sie in eigene Kredite transformieren kann und legt diese überschüssigen Finanzmittel auf dem Kapitalmarkt zum dortigen Zinssatz $R$ an. ${ }^{2}$

Zu Beginn der Entscheidungsperiode setzt die Bank die Zinsen für Kredite und Depositen fest. Die Unsicherheit hinsichtlich des Angebots an Depositen erfordert u.U. den Ausgleich der Bilanz unter Rückgriff auf den inländischen Kapitalmarkt. Die Bank agiert als ein Zinssetzer auf dem Kreditmarkt. Die Kreditnachfrage ist eine negative Funktion der Kreditzinsen. Es gilt:

(2) $L=L\left(R_{L}\right)$ sowie $L^{\prime}\left(R_{L}\right)<0 .^{3}$

Die Höhe der während der Periode zur Verfügung stehenden Depositen ist unsicher. Entsprechend wird die Angebotsfunktion an Depositen als positive Funktion des Depositenzinssatzes $R_{D}$ modelliert, additiv erweitert um einen Zufallsterm $(\mu)$, dessen Dichtefunktion $g(\mu)$ bekannt ist. Der Erwartungswert von $\mu$ in seinen Grenzen $(-m, \infty)$ ist Null.

Das Angebot an Depositen wird durch folgende Funktion beschrieben:

$$
D^{*}=D\left(R_{D}\right)+\mu \quad \text { und } \quad D^{\prime}\left(R_{D}\right)>0 .
$$

Die Bank strebt die Maximierung des Erwartungsnutzens ihres Gewinns $\pi$ an. Sei $U(\pi)$ eine von Neumann-Morgenstern Nutzenfunktion ${ }^{4}$ mit dem Argument des Periodenendgewinns und den Eigenschaften $U^{\prime}(\pi)>0$ sowie $U^{\prime \prime}(\pi) \leqq 0$.

Das Optimierungsproblem der Bank lautet damit:

$$
\underset{R_{L}, R_{D}}{\text { Maximiere }} \bar{U}=E[U(\pi)]=\int_{-m}^{\infty} U(\pi) g(\mu) d \mu
$$

1 Von Mindestreserveanforderungen wird abstrahiert, da sie das Modell komplexer machen, ohne die Ergebnisse qualitativ zu beeinflussen.

2 Empirisch erwiesen ist, daß großßere Banken ihre Zinsen langsamer und kleinere Banken ihre Zinsen schneller an die Kreditmarktzinsen anpassen. Vgl. hierzu Weth $(2002,22)$. Davon wird in diesem Modell abstrahiert.

3 Ein Apostroph kennzeichnet die erste, partielle Ableitung einer Funktion, entsprechend verdeutlichen zwei Apostrophe die zweite, partielle Ableitung.

4 Die von Neumann-Morgenstern Nutzenfunktion beschreibt eine Nutzenfunktion bzw. vielmehr eine Klasse von Präferenz-Beziehungen, die den erwarteten Nutzen generieren. Die zentrale Aussage lautet, daß der Entscheidungstrăger sich indifferent verhălt zwischen dem Erhalt eines gegebenen Guterbundels oder einem Spiel, dessen Ergebnis denselben Erwartungswert aufweist. Vgl. hierzu http://economics.about.com. 
u.B.v. $L+B=D^{*}+E$.

Der Bankgewinn setzt sich aus vier Elementen zusammen. Zum einen fließen der Bank die Zinseinnahmen aus erteilten Krediten zu. Hieraus müssen die Zinszahlungen für erhaltene Depositen wie auch die Prämie für das Einlagensicherungssystem beglichen werden. Den vierten Gewinnbestandteil stellt die Nettoposition der Bank auf dem Kapitalmarkt dar: Verfügt sie über Guthaben, so werden Zinseinnahmen generiert; nimmt sie eine Passivposition ein, so müssen entsprechende Zins- und Tilgungszahlungen geleistet werden.

Der Bankgewinn ist definiert als:

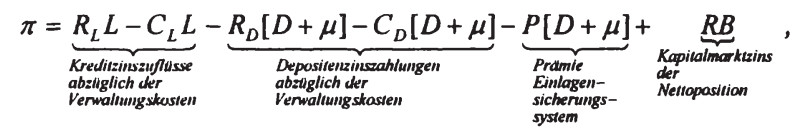

wobei:

$$
\begin{array}{ll}
\mathrm{P} & \text { zu zahlende Prämie an das Einlagensicherungssystem, } \\
C_{L} \text { bzw. } C_{D}= & \text { administrative Kosten der Bereitstellung und } \\
& \text { Verwaltung von Krediten bzw. Depositen, } \\
\mathrm{R} \quad & \text { Kapitalmarktzins. }
\end{array}
$$

Durch Einfügen der Gleichung (1) in die Gewinnfunktion folgt:

$$
\pi=R_{L} L-C_{L} L-R_{D}[D+\mu]-C_{D}[D+\mu]-P[D+\mu]+R[D+\mu+E-L] .
$$

Folglich kann die Gewinnfunktion als unbedingte Zielfunktion formuliert werden:

$$
\underset{R_{L}, R_{D}}{\text { Maximiere }} \int_{-m}^{\infty} U\left\{R_{L} L-C_{L} L-R_{D}[D+\mu]-C_{D}[D+\mu]-P[D+\mu]+R[D+\mu+E-L]\right\} g(\mu) d \mu .
$$

\section{Die Lösungen des Modells}

Da es sich um ein Optimierungsproblem handelt, erfolgt die Lösung des Modells über die Ermittlung der partiellen Ableitungen. Somit lauten die Bedingungen erster Ordnung für das Gewinnmaximum: 

(8) $\frac{\partial \bar{U}}{\partial R_{L}}=\bar{U}_{L}=E\left\{U^{\prime}(\pi)\left[L+L^{\prime}\left(R_{L}-C_{L}-R\right)\right]\right\}=0,{ }^{1}$
(9) $\frac{\partial \bar{U}}{\partial R_{D}}=\bar{U}_{D}=E\left\{U^{\prime}(\pi)\left[-D-\mu+D^{\prime}\left(R-R_{D}-C_{D}-P\right)\right]\right\}=0$.

Die Bedingungen zweiter Ordnung für ein Maximum lauten:

(10) $\bar{U}_{L}=E\left\{U^{\prime}(\pi)\left[2 L^{\prime}+L^{\prime \prime}\left(R_{L}-C_{L}-R\right)\right]+U^{\prime \prime}(\pi)\left(\pi_{L}\right)^{2}\right\}<0$,

(11) $\bar{U}_{D D}=E\left\{U^{\prime}(\pi)\left[-2 D^{\prime}+D^{\prime \prime}\left(R-R_{D}-C_{D}-P\right)\right]+U^{\prime \prime}(\pi)\left(\pi_{D}\right)^{2}\right\}<0$,

(12) $H=\bar{U}_{L} \bar{U}_{D D}-\bar{U}_{L D}^{2}>0$.

Dabei gilt: $U_{L D}=E\left[\pi_{L}\right] E\left[U^{\prime \prime}(\pi) \pi_{D}\right]=0$, da $E\left[\pi_{L}\right]=0 .^{2}$

Damit kann die erste partielle Ableitung der Gewinnfunktion nach dem Argument $R_{L}$ (Kreditzinsen) [Gleichung (8)] neu formuliert werden als:

(13) $\bar{U}_{L}=E\left[U^{\prime}(\pi)\right] L+E\left[U^{\prime}(\pi)\right] L^{\prime}\left(R_{L}-C_{L}-R\right)=0$.

Aus Gleichung (8) ist ersichtlich, daß $\left(R_{L}-C_{L}-R\right)>0$ erfüllt sein muß, um einen zulässigen Wert für $R_{L}$, also den Kreditzins, zu erhalten.

In ähnlicher Weise kann Gleichung (9) erfaßt werden, also die Gewinnmaximierungsbedingung erster Ordnung in Abhängigkeit vom Depositenzinssatz $R_{D}$ :

(14) $\bar{U}_{D}=E\left[U^{\prime}(\pi)(-D-\mu)\right]+E\left[U^{\prime}(\pi)\right] D^{\prime}\left(R-R_{D}-C_{D}-P\right)=0$.

Der erste Term der Gleichung (14) ist negativ, da $U^{\prime}(\pi)>0$ und $(-D-\mu)<0$.

Folglich muß die Ungleichung $\left.\left(R-R_{D}-C_{D}-P\right)>0\right)$ erfüllt sein, um einen zulässigen Wert für $R_{D}$ zu erhalten.

Eine zulässige Lösung verlangt also, daß der Spread zwischen $R_{L}$ und $R_{D}$ eindeutig größer sein muß als die Summe aus der Sicherungsprämie $P$ sowie den Verwaltungskosten für die Kredite und Depositen $\left(C_{L}\right.$ und $\left.C_{D}\right)$.

1 Die Einfach-Indizierung mit einem Buchstaben verweist auf die erste partielle Differenzierung nach dem entsprechenden Zinssatz, also $L$ für Kreditzins und $D$ fur Depositenzins. Die doppelte Indizierung verweist auf die zweite partielle Differenzierung.

2 Vgl. die notwendige Bedingung fur $R_{L}$. 


\subsubsection{Variation der administrativen Kosten}

Nun sollen die Auswirkungen protektionistischer Maßnahmen in das Modell integriert werden. Diese sollen kostenerhöhend wirken, bspw. durch eine gesetzliche Regelung, wonach ausländische Banken nur Inländer zu einem festgesetzten Lohn beschäftigen dürfen oder ausländische Banken höhere Rücklagen vorweisen müssen. Dieser Umstand wird ins Modell eingefügt, indem die administrativen Kosten erhöht werden. Eine Veränderung der administrativen Kosten für die Depositen $\left(C_{D}\right)$ hat nachstehende Veränderungen zur Folge. Die Anpassung der Bedingungen erster Ordnung hinsichtlich $C_{D}$ :

$$
\begin{aligned}
& \text { (15) } \frac{\partial R_{D}}{\partial C_{D}}=\frac{D^{\prime} E\left[U^{\prime}(\pi)\right]-E\left\{U^{\prime \prime}(\pi)(-D-\mu)\left[-D-\mu+D^{\prime}\left(R-R_{D}-C_{D}-P\right)\right]\right\}}{\bar{U}_{D D}}, \\
& \text { (16) } \frac{\partial R_{L}}{\partial C_{D}}=\frac{-E\left\{U^{\prime \prime}(\pi)(-D-\mu)\right\} E\left[L+L^{\prime}\left(R_{L}-C_{L}-R\right)\right]}{\bar{U}_{L}} .
\end{aligned}
$$

Gleichung (16) zeigt, daß der optimale Kreditzins unabhängig ist von den Veränderungen der Verwaltungskosten in Zusammenhang mit der Bereitstellung und Verwaltung von Depositen. Nur die Veränderungen in Gleichung (15) bestimmen den Gesamteffekt.

Der erste Zählerterm ist positiv. Um das Vorzeichen des zweiten Terms zu ermitteln, sind weitere Umformungen nötig:

$$
\begin{aligned}
& E\left\{U^{\prime \prime}(\pi)(-D-\mu)\left[-D-\mu+D^{\prime}\left(R-R_{D}-C_{D}-P\right)\right]\right\} \\
& =E\left\{U^{\prime \prime}(\pi)\left[-D-\mu+D^{\prime}\left(R-R_{D}-C_{D}-P\right)\right]^{2}\right\}-D^{\prime}\left(R-R_{D}-C_{D}-P\right) . \\
& \times E\left\{U^{\prime \prime}(\pi)\left[\left(-D-\mu+D^{\prime}\left(R-R_{D}-C_{D}\right)-P\right]\right\}\right.
\end{aligned}
$$

Gleichung (17) ist nicht allgemein determiniert. Wird aber ein konstant bleibendes, risikoaverses Verhalten ${ }^{1}$ der Bank unterstellt, so ist ein positiver Zusammenhang feststellbar.

Der erste Term von Gleichung (17) ist eindeutig negativ. Bei konstant vorherrschender Risikoaversion der Bank ist folglich Gleichung (15) negativ. Die inhaltliche Interpretation der mathematischen Zusammenhänge lautet, daß die Bank steigende Verwaltungsgebühren an ihre Kunden über sinkende Depositenzinsen weiterreicht. Ein steigendes $C_{D}$ führt also dazu, daß die Depositenzinsen fallen und die Zinsmargen der Bank steigen.

1 Zarruck unternimmt an dieser Stelle eine Fallunterscheidung hinsichtlich des Bankenverhaltens: abnehmende Risikoaversion $(D A R A=$ Decreasing Absolute Risk Aversion), konstante Risikoaversion $(C A R A=$ Constant Absolute Risk Aversion) und zunehmende Risikoaversion $(I A R A=$ Increasing Absolute Risk Aversion). Diese Indizes wurden ursprunglich von Arrow (1964) und Pratt (1964) entwickelt. Vgl. hierzu insbesondere Zarruck $(1989,801)$. 
Eine parallele Analysemöglichkeit liefert die Betrachtung der Risikoprämie Z. Die Zahlung der Risikoprämie führt dazu, daß die Bank indifferent wird zwischen einem sicheren Gewinn bzw. Erwartungsnutzen $(E[\pi]-Z)$ und dem zufallsabhängigen Gewinn $\pi$.

Die Risikoprämie $Z$ sei abhängig vom Erwartungswert und der Varianz des Gewinns: $Z=Z(E[\pi], \operatorname{Var}(\pi))$.

Damit wirkt sich die Veränderung von $C_{D}$ auf die Risikoprämie folgendermaßen aus:

$$
\frac{d Z}{d C_{D}}=\frac{\partial Z}{\partial E(\pi)} \frac{\partial E[\pi]}{\partial D_{D}}+\frac{\partial Z}{\partial \operatorname{Var}(\pi)} \frac{\partial \operatorname{Var}(\pi)}{\partial C_{D}}
$$

Diese Gleichung erfaßt die Auswirkungen einer Änderung von $C_{D}$ hinsichtlich des durchschnittlichen Gewinneffekts und des Risikoeffekts von $Z$. Es folgt:

(20) $\frac{\partial \operatorname{Var}(\pi)}{\partial C_{D}}=-2\left(R-R_{D}-C_{D}-P\right) \operatorname{Var}(\mu)<0$.

Die Gleichungen (19) und (20) besagen, daß durch eine Erhöhung der Depositenzinsen $\left(C_{D}\right)$ dazu führt, daß sowohl der Erwartungswert wie auch die Varianz der Bankgewinne reduziert werden.

Da der Ausdruck $\frac{\partial Z}{\partial \operatorname{Var}(\pi)}>0$, ist der zweite Term von Gleichung (18) negativ für

jede risikoaverse Nutzenfunktion. Bei einem gleichbleibenden oder steigenden Niveau der Risikoaversion ist der Mittelwert des Gewinneffekts auf $Z$ durch eine Veränderung von $C_{D}$ Null (CARA) oder negativ (IARA) und somit gilt: $\frac{\partial Z}{\partial C_{D}}<0$

Da die Depositenzinsen gemeinsam mit der Risikoprämie steigen, gilt $\frac{\partial R_{D}}{\partial C_{D}}<0$ :

Auch in dieser Betrachtungsweise werden bei steigenden Verwaltungskosten die Depositenzinsen gesenkt. ${ }^{\prime}$ Statt die Maximierung des Gewinns zu verfolgen,

Sollte die Risikoaversion der Bank sinken (DARA), so ist der Effekt steigender Verwaltungskosten nicht eindeutig bestimmbar, vgl. hierzu Zarruk 1989, 801 ff. 
erfolgte hier die Analyse des Bankenverhaltens anhand der Risikoprämie $Z$. Auch diese Vorgehensweise bestätigte die vorhergehenden Ergebnisse.

Betrachten wir die Kreditseite, so ergeben sich für die Gewinnmaximierungsbedingungen erster Ordnung analog zur Darstellung der Depositenseite:

$$
\begin{aligned}
& \frac{\partial R_{D}}{\partial C_{L}}=\frac{L E\left\{U^{\prime \prime}(\pi)\left[-D-\mu+D^{\prime}\left(R-R_{D}-C_{D}-P\right)\right]\right\}}{\bar{U}_{D D}}, \\
& \frac{\partial R_{L}}{\partial C_{L}}=\frac{L^{\prime} E\left[U^{\prime}(\pi)\right]+L E\left[U^{\prime \prime}(\pi)\right] E\left[L+L^{\prime}\left(R_{L}-C_{L}-R\right)\right]}{\bar{U}_{L}}>0 .
\end{aligned}
$$

Ähnliche Überlegungen wie bei der Variation der Verwaltungskosten sind auch für die Veränderung der Prämien des Einlagensicherungssystems zutreffend. Die Zinsmargen sind eine positive Funktion der Einlagensicherungsprämien. Die Differenzierung der Bedingung erster Ordnung für ein Maximum des Erwartungsnutzens hinsichtlich des Arguments $P$ liefert:

$$
\begin{aligned}
& \frac{\partial R_{L}}{\partial P}=\frac{-E\left\{U^{\prime \prime}(\pi)(-D-\mu)\right\} E\left[L+L^{\prime}\left(R_{L}-C_{L} R\right)\right]}{\bar{U}_{L L}} \\
& \frac{\partial R_{D}}{\partial P}=\frac{D^{\prime} E\left[U^{\prime}(\pi)\right]-E\left\{U^{\prime \prime}(\pi)(-D-\mu)\left[-D-\mu+D^{\prime}\left(R-R_{D}-C_{D}-P\right)\right]\right\}}{\bar{U}_{D D}} .
\end{aligned}
$$

Die Ausdrücke für $\frac{\partial R_{L}}{\partial P}$ und $\frac{\partial R_{D}}{\partial P}$ sind identisch mit denen der Gleichungen (15) und (16).

Somit haben Veränderungen der Prämie, die an das Einlagensicherungssystem zu entrichten ist, dieselben Folgen wie Veränderungen der Verwaltungskosten: Die Kosten der Bank steigen. Um diesen Druck abzufangen, senken sie die Depositenzinsen.

\subsubsection{Implikationen des Modells}

Das Modell verdeutlicht das Verhalten der Bank als ein gewinnmaximierendes Unternehmen. Als solches richtet es seine Zinsgestaltung an dem risikolosen Geldmarkt- (und damit Notenbank-)Zins aus, die es mit Auf- oder Abschlägen zur Deckung aller Betriebskosten versieht.

Steigende Verwaltungskosten oder Versicherungsprämien nehmen direkten Einfluß auf die Höhe der Zinsen. Diese Erkenntnis bestätigt die Hypothese, daß Regulierungen, die Kosten verursachen, ihren direkten Niederschlag in höheren Zinsmargen finden. Dieser Umstand trifft auf nahezu alle denkbaren Regulierun- 
gen zu, ob sie nun direkt kostenerhöhend wirken oder aber auch nur indirekt durch einen erhöhten Umsetzungs- und Kontrollbedarf. Werden diese Regulierungen nur gegenüber ausländischen Banken angewendet, sind sie also protektionistisch, so erhöhen sie deren Produktionskosten. Dieser Umstand muß durch die entsprechend hohe Setzung der Zinsen kompensiert werden. Eventuell zunächst vorhandene Wettbewerbsvorteile werden so verringert bzw. eliminiert. Die heimischen Banken können ihr bisheriges Verhalten beibehalten, die ausländische Konkurrenz kann ihre Geschäftsmodelle nicht vergleichbar umsetzen. Die Erweiterung des Modells um die Betrachtung der zu zahlenden Prämie an das Einlagensicherungssystem verdeutlicht ebenfalls den direkten Einfluß auf die Zinsmargen: Steigende Prämiensätze werden direkt an die Bankkunden weitergereicht.

Entsprechend wird hinsichtlich der in Kapitel vier zu untersuchenden Regulierungen des Bankenmarktes und internationalen Finanzverkehrs davon ausgegangen, daß sie tendenziell zinsmargenerhöhend wirken. Zunächst jedoch erfolgt eine kurze Beschreibung der aktuell vorliegenden Zinsmargendaten, die als Indikator zur Messung des Regulierungsniveaus verwendet werden.

\subsection{Zinsmargen: Aktuelle Daten und ihre Entwicklung}

\subsubsection{Gesamtentwicklung der Zinsmargen}

Die in dieser Studie verwendeten Daten zur Zinsmargenhöhe stammen aus Untersuchungen der WTO.' Zur Verfügung stehen die durchschnittlichen Zinsmargen für die zwei Perioden 1998 bis 2000 sowie 2001 bis 2003. Leider weisen die Datensätze Lücken auf. Deshalb werden zunächst nur die Länder betrachtet, bei denen Angaben für beide Zeiträume vorliegen.

Tab. 3-2: Statistik der Nettozinsmargen

(für Länder in beiden Untersuchungsperioden)

\begin{tabular}{l|l|l|l|l|l|} 
& Länder & Mittelwert & $\begin{array}{l}\text { Standard- } \\
\text { abweichung }\end{array}$ & Minimum & Maximum \\
\hline $1998-2000$ & 54 & 0,0484148 & 0,0258689 & 0,0119 & 0,1161 \\
$2001-2003$ & 54 & 0,0334056 & 0,0235867 & 0,0060 & 0,1430 \\
\hline Differenzen & $-0,0150092$ & $-0,0022822$ & $-0,0059$ & $+0,0269$
\end{tabular}

Im Durchschnitt sanken die Nettozinsmargen im Verlauf von funf Jahren von 0,0484 auf den Wert von 0,0334 . Auch die Standardabweichung, also die Varianz der Zinsmargen, hat sich von 0,0259 auf 0,0236 verringert. Dieselbe Entwicklung ist bei den Tiefstwerten zu beobachten: Sie sanken von 0,0119 auf 0,006. Bei den

1 Die Angaben zur Herkunft der Daten werden in Kapitel 4.1.1. Die Datenbasis konkretisiert. 
Höchstwerten erfolgte eine Steigerung, die auf den Ausreißer Ghana zurückzuführen ist. Entsprechend steigt der Höchstwert von 0,1161 auf 0,1430. Bei Ausschluß Ghanas aus der Betrachtung ergibt sich ein eindeutigeres Bild: Mittelwert, Standardabweichung und Höchstwert haben sich im Verlauf von nur wenigen Jahren erheblich verringert.

\section{Tab. 3-3: Statistik der Nettozinsmargen (ohne Ghana)}

\begin{tabular}{l|l|l|l|l|l|} 
& Länder & Mittelwert & $\begin{array}{l}\text { Standard- } \\
\text { abweichung }\end{array}$ & Minimum & Maximum \\
\hline $1998-2000$ & 53 & 0,0471377 & 0,0243373 & 0,0119 & 0,1074 \\
$2001-2003$ & 53 & 0,0313377 & 0,0182125 & 0,0060 & 0,0940 \\
\hline Differenzen & $-0,0158$ & $-0,0061248$ & $-0,0059$ & $-0,0134$
\end{tabular}

Eine graphische Darstellung verdeutlicht den Trend zur Reduzierung der Nettozinsmargen. Die Abbildung 3-2 dokumentiert, daß in der überwiegenden Anzahl der Länder die Nettozinsmargen gesunken sind. Es gibt nur 5 Ausnahmen, in denen die Zinsmargen gestiegen sind: Ghana, Jordanien, Bolivien, Finnland sowie Panama.

Diese bisherigen Darstellungen erfassen nicht alle in den Umfragen ermittelten Zinsmargenwerte. Es wurden zunächst nur die Daten der Länder verwendet, für die auch in beiden Perioden Informationen vorliegen. Die Berücksichtigung aller vorliegenden Länderdaten, also auch der Länder, bei denen nur Angaben für eine Untersuchungsperiode vorliegen, führt zu folgenden Ergebnissen: Die durchschnittliche Nettozinsmarge (ohne Gewichtung) weist für den Zeitraum von 1998 bis 2000 eine Höhe von 0,0474 . auf. Im folgenden Zeitraum (2001 - 2003) beträgt die durchschnittliche Nettozinsmarge nur noch 0,0399. Die Tabelle 3-4 stellt diese Daten zusammen. Deutlich zu erkennen ist das Sinken des Mittelwerts, wobei sich die Varianz der Daten deutlich erhöht hat. Dieses nicht eindeutige Bild läßt sich durch den Einbezug von 49 neuen Ländern in die Analyse erklären. ${ }^{1}$ Die neu hinzugekommenen Länder sind tendenziell eher wenig entwickelt; sie weisen entsprechend hohe Zinsmargen auf. Dadurch liefert eine Betrachtung der Durchschnittswerte keine eindeutigen Ergebnisse; der Grad an Heterogenität hat sich erheblich erhöht. Aufgrund der großen Heterogenität der Länder hinsichtlich ihres Entwicklungsstandes bietet sich die Darstellung der Zinsmargen unter Bezugnahme auf das Pro-Kopf-Einkommen an. Ergänzend wurde eine logarithmische Regressionslinie wie auch das Bestimmtheitsmaß berechnet. ${ }^{2}$

1 Hierbei handelt es sich uberwiegend um Entwicklungslănder. Eine vollstăndige Liste der Lănder ist dem Anhang A2 zu entnehmen.

2 Eine Gesamtubersicht der Datensătze Zinsmarge und Pro-Kopf-Einkommen findet sich im Anhang A3. 
Abb. 3-2: Veränderung der absoluten Zinsmargen im Zeitablauf:

Vergleich der Periode 1998-2000 vs. Periode 2001-2003
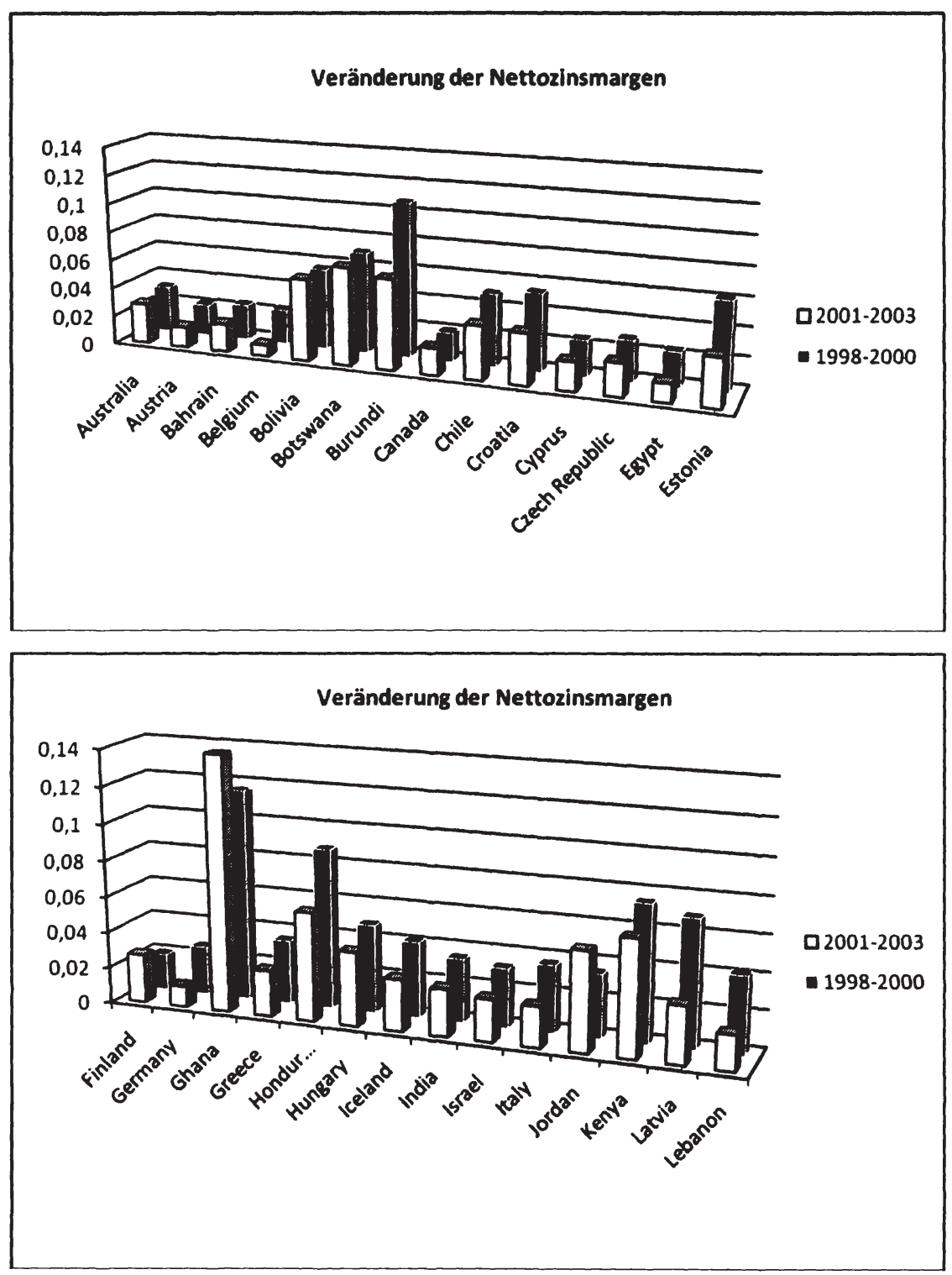
Abb. 3-2: Veränderung der Zinsmargen im Zeitablauf (Forts.)
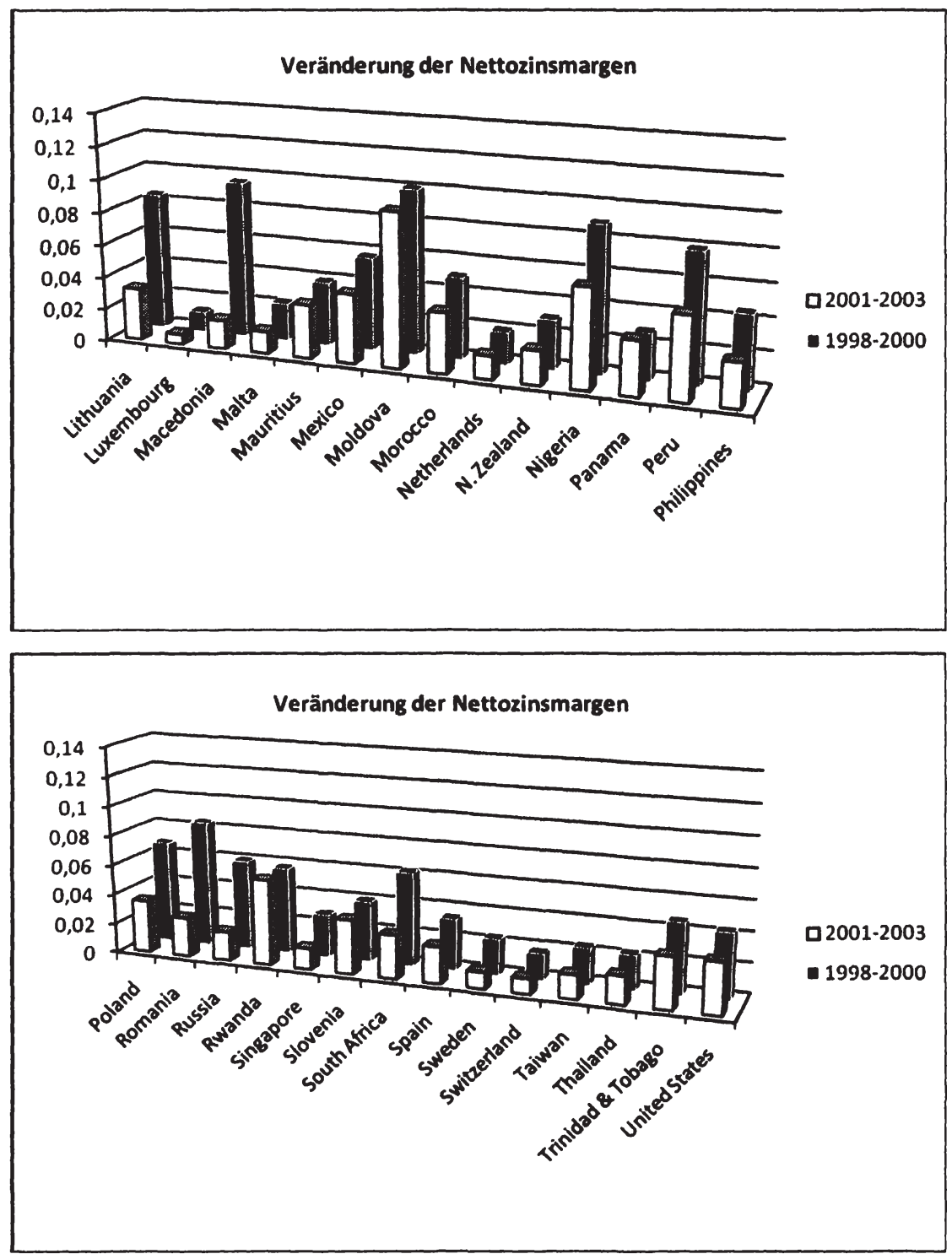
Tab. 3-4: Statistik der Nettozinsmargen (alle Länder)

\begin{tabular}{l|l|l|l|l|l|} 
& Länder & Mittelwert & $\begin{array}{l}\text { Standard- } \\
\text { abweichung }\end{array}$ & Minimum & Maximum \\
\hline $1998-2000$ & 70 & 0,0474286 & 0,0258984 & 0,0119 & 0,1161 \\
$2001-2003$ & 119 & 0,0398979 & 0,0276917 & 0,0004 & 0,1480 \\
\hline Differenzen & +49 & $-0,0075307$ & $+0,0017933$ & $-0,0115$ & $+0,0319$
\end{tabular}

Wie der Abbildung 3-3 zu entnehmen ist, liegt eine eindeutige Beziehung zwischen Pro-Kopf-Einkommen und Nettozinsmargen vor. In der Regel weisen Lănder mit hohem Pro-Kopf-Einkommen auch eine niedrige Zinsmarge auf. Zinsmargen oberhalb des Wertes von 0,06 weisen nur Länder mit einem Pro-KopfEinkommen von unter 10.000 US-Dollar pro Jahr auf. Länder, die sich also um Wohlstandssteigerung und höhere Pro-Kopf-Einkommen bemühen, sollten ihren Fokus auch auf die Senkung der Nettozinsmargen legen. Dieses ist über eine stärkere Liberalisierung, weitreichende Integration in die internationalen Kapitalmärkte und eine konkurrenzfähige Struktur ihrer Bankenmärkte zu erreichen, wie die Analyse des vierten Kapitels zeigen wird.

Um einen detaillierteren Einblick in die Verteilung der Zinsmargen-EinkommensRelation zu erhalten, werden die Länder gemäß ihres Pro-Kopf-Einkommens in Klassen unterteilt. In der Regel weisen Länder mit ähnlich hohem Pro-Kopf-Einkommen auch einen ähnlichen Entwicklungsstand ihrer Wirtschaft auf und, wie das folgende Kapitel zeigen wird, auch ähnliche Zinssätze. Die Gruppierung der Länder nach Einkommensklassen erleichtert die Vergleichbarkeit und ermöglicht es, Zusammenhänge zwischen der Entwicklung der Zinsmargen und der Einkommen aufzuzeigen.

Untersucht werden die beiden Zeiträume 1998-2000 sowie 2001-2003. Es werden nur die Länder berücksichtigt, deren Nettozinsmargenwerte in beiden Perioden vorliegen. Neben der durchschnittlichen Nettozinsmarge je Einkommensgruppe werden auch die Maximum- und Minimumwerte angegeben, um die Spannweite der Schwankungen um den Durchschnittswert aufzuzeigen. Dieselbe Vorgehensweise wird bei der Betrachtung des Pro-Kopf-Einkommens angewendet. Anschließend wird die absolute wie auch die prozentuale Veränderung der Durchschnittswerte der Nettozinsmargen (NIM) und des Pro-Kopf-Einkommens (PKE) zwischen beiden Perioden ermittelt. 
Abb. 3-3: Zinsmargen und Pro-Kopf-Einkommen
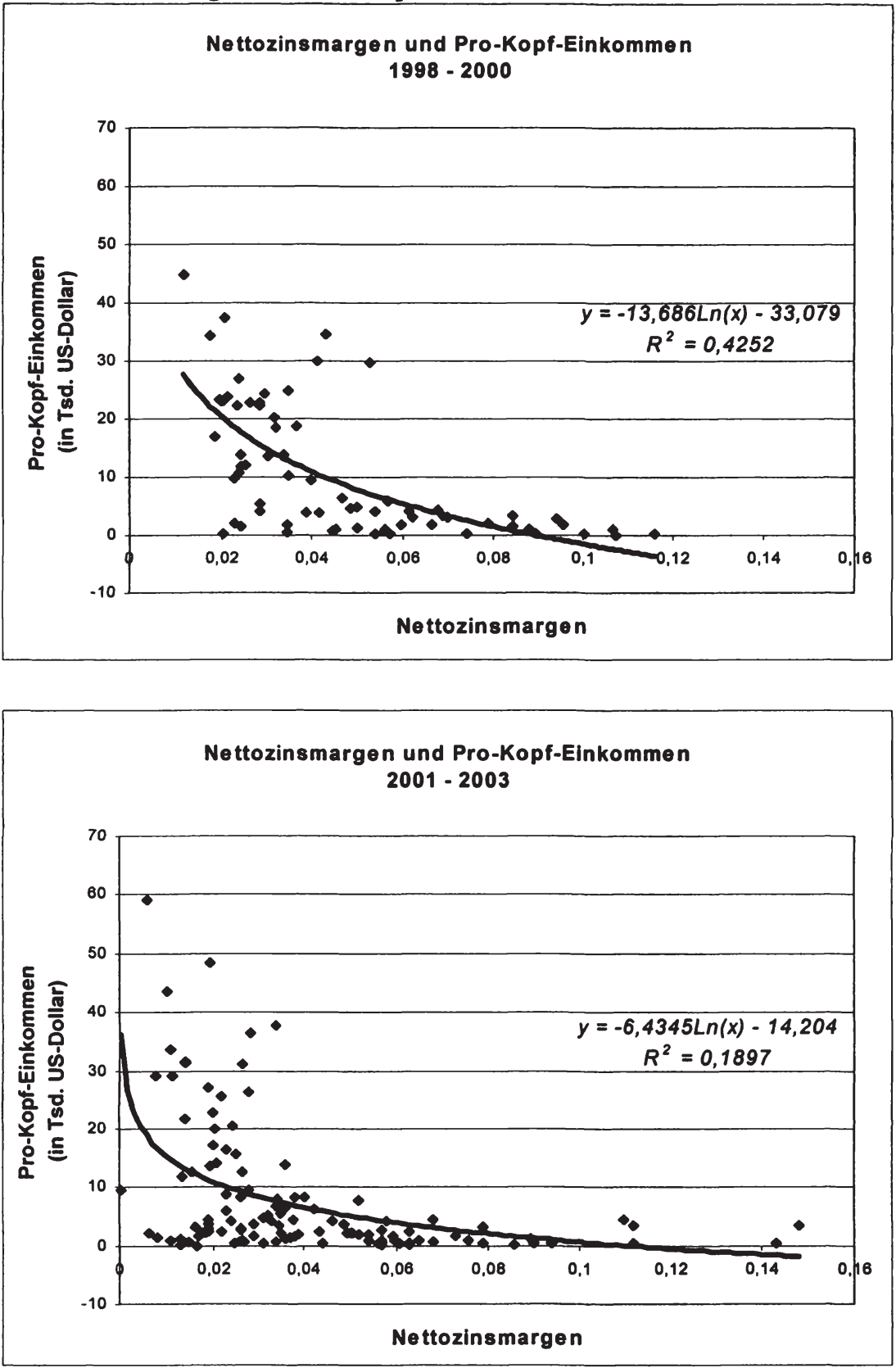


\subsubsection{Ländergruppe I (Einkommensklasse $>15.000$ US-Dollar)}

Als Gruppe der Industrienationen werden Länder mit einem Pro-Kopf-Einkommen höher als 15.000 US-Dollar betrachtet. Diese Gruppe setzt sich überwiegend aus europäischen Ländern zusammen, ergänzt um die Industrienationen USA, Kanada, Japan, Israel und auch Kuwait. Die Betrachtung der Gruppe der entwickelten und vergleichsweise wohlhabenden Länder liefert folgendes Ergebnis:

Tab. 3-5: Ergebnistableau (> 15.000 US-Dollar)

\begin{tabular}{ll|cc} 
& & $1998-2000$ & $2001-2003$ \\
\hline \multirow{2}{*}{ NIM } & Maximum der Gruppe & 0,0434 & 0,0338 \\
& Minimum der Gruppe & 0,0119 & 0,006 \\
& Durchschnittswert & 0,0266 & 0,0179 \\
\cline { 2 - 3 } & Differenz (Durchschnitt) & \multicolumn{2}{|c}{$-0,0087$} \\
& Differenz prozentual & \multicolumn{2}{|c}{$-32,7$ v.H. } \\
\hline \multirow{2}{*}{ PKE } & Maximum der Gruppe & 44.757 US-\$ & 59.143 US-\$ \\
& Minimum der Gruppe & 18.358 US $-\$$ & 16.481 US-\$ \\
& Durchschnittswert & 25.941 US-\$ & 31.969 US-\$ \\
\cline { 2 - 3 } & Differenz (Durchschnitt) & \multicolumn{2}{|c}{+6.028 US-\$ } \\
& Differenz prozentual & \multicolumn{2}{|c}{$+23,2$ v.H. } \\
\hline Länder & & \multicolumn{2}{|c}{15}
\end{tabular}

Innerhalb nur weniger Jahre sank die Nettozinsmarge sehr deutlich um 32,7 Prozent (von 0,0266 auf 0,0179), während das durchschnittliche (Jahres-)Pro-KopfEinkommen um 23,2 Prozent von 25.941 auf 31.969 US-Dollar stieg. Länder dieser Einkommensklasse weisen ein erwartungsgemäß hohes Wachstum des ProKopf-Einkommens wie auch eine hohe Reduktion der Nettozinsmargen auf. Dies sind Indizien dafür, daß sich insbesondere die hochentwickelten Länder am internationalen Kapitalmarkt betätigen. Um die Vorteile eines globalen Kapitalmarktes in Form günstiger Konditionen bei der Kapitalbeschaffung zu nutzen, bspw. günstige Preise für Finanzdienstleistungen, haben diese Länder in einem hohen Ausmaß Beschränkungen auf ihren Bankenmärkten reduziert. Der internationale Wettbewerbsdruck ließ in diesen Ländern einen leistungsfähigen Finanzsektor

Verănderung der Durchschnittswerte im Zeitablauf. 
Abb. 3-4: Vergleich der Zinsmargen (Einkommensklasse $>15.000$ US-Dollar)
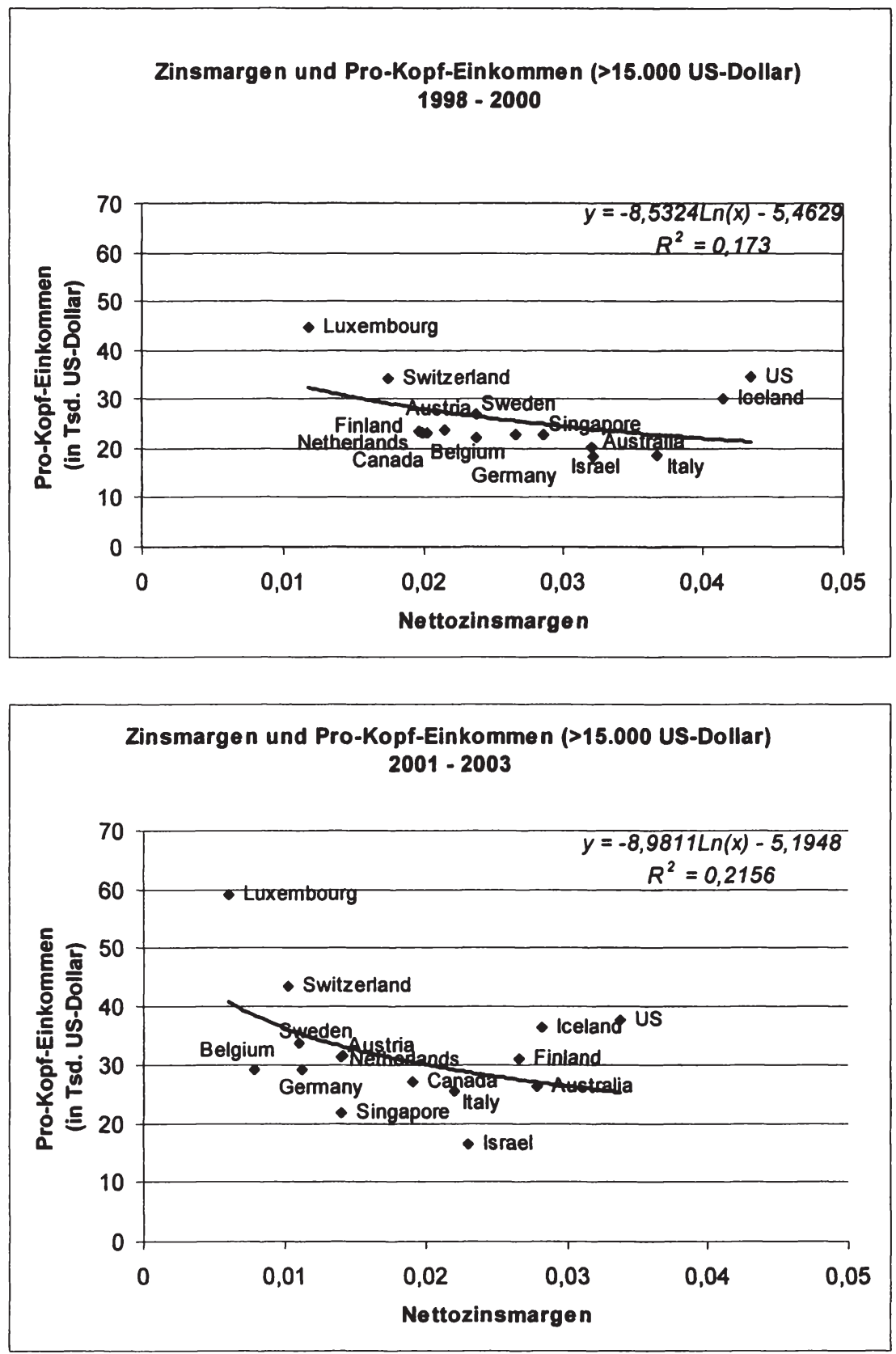
entstehen. Entsprechend profitieren sie von höheren Wachstumsraten der Wirtschaft (Francois / Eschenbach 2002, 10).

Graphisch äußert sich die Reduktion der durchschnittlichen Nettozinsmargen dadurch, daß sich das Punktefeld in seiner Gesamtheit deutlich zur Ordinate hin verlagert. Das Punktefeld weist aber innerhalb dieser Bewegung eine größere Streuung auf, die Verringerung der Zinsmargen hat sich also in unterschiedlich starkem Ausmaß vollzogen.

\subsubsection{Ländergruppe II (Einkommensklasse 5.000 - 14.999 US-Dollar)}

In der Gruppe der Länder mit einem Pro-Kopf-Einkommen zwischen 5.000 und 14.999 US-Dollar treffen Industrienationen wie Spanien oder Taiwan auf Schwellen- und Transformationsländer wie die Tschechische Republik.

Tab. 3-6: Ergebnistableau (5.000 - 14.999 US-Dollar)

\begin{tabular}{ll|cc} 
& & $1998-2000$ & $2001-2003$ \\
\hline \multirow{2}{*}{ NIM } & Maximum der Gruppe & 0,0570 & 0,0424 \\
& Minimum der Gruppe & 0,0242 & 0,0135 \\
& Durchschnittswert & 0,0335 & 0,0249 \\
\cline { 2 - 3 } & Differenz (Durchschnitt) & \multicolumn{2}{|c}{$-0,0086$} \\
& Differenz prozentual & \multicolumn{2}{|c}{$-25,67$ v.H. } \\
\hline \multirow{3}{*}{ PKE } & Maximum der Gruppe & 13.879 US-\$ $\$$ & 20.4045 US-\$ \\
& Minimum der Gruppe & 5.423 US-\$ & 6.248 US-\$ \\
& Durchschnittswert & 10.232 US-\$ & 13.466 US-\$ \\
\cline { 2 - 3 } & Differenz (Durchschnitt) & \multicolumn{2}{|c}{+3.234 US-\$ $\$$} \\
& Differenz prozentual & \multicolumn{2}{|c}{11,6 v.H. } \\
\hline Länder & & \multicolumn{2}{|c}{11}
\end{tabular}

Die Nettozinsmarge sank in dieser Gruppe im Durchschnitt um 25,67 Prozent (von 0,0335 auf 0,0249). Gleichzeitig stieg das Pro-Kopf-Einkommen in dieser sehr heterogenen Gruppe, und Spanien erreichte im zweiten Untersuchungszeitraum sogar eine Zunahme des Pro-Kopf-Einkommens auf über 20.000 US-Dollar.

Auch in dieser Einkommensklasse läßt sich graphisch deutlich die Verlagerung des Schwerpunktes der Punktewolke zur Ordinate hin konstatieren. Allerdings gelingt nicht allen Ländern die Reduktion der Zinsmargen in ähnlichem Ausmaß. Slowenien, Mexiko sowie Trinidad und Tobago gelingen nur sehr geringe 
Abb. 3-5: Vergleich der Zinsmargen (Einkommensklasse 5.000 - 14.999 USDollar)
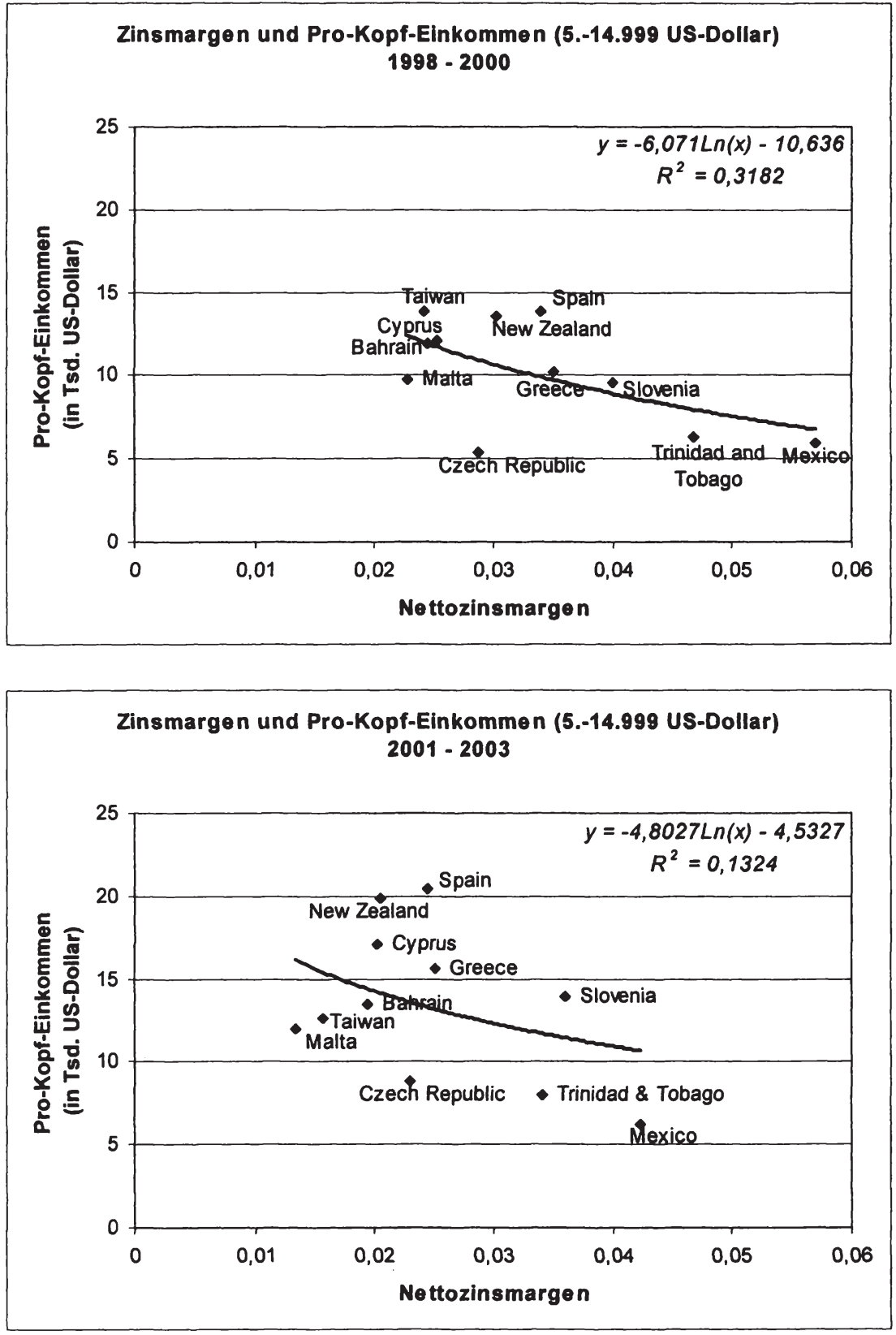
Senkungen der Zinsmargen, ihre Erfolge fallen unterdurchschnittlich aus. Die übrigen Länder dieser Einkommensklasse gruppieren sich um den Zinsmargenwert von 0,02 .

\subsubsection{Ländergruppe III (Einkommensklasse 1.000 - 4.999 US-Dollar)}

Die Einkommensklasse der Länder mit einem Pro-Kopf-Einkommen zwischen 1.000 und 4.999 US-Dollar weist im Zeitablauf Länder aller Kontinente auf. Zum einen gehören einige mittel- und osteuropäische Länder (Transformationsländer) in diese Gruppe, z.B. Lettland, Litauen, Estland, Polen. Diese Nationen verzeichnen eine beachtliche Einkommenserhöhung und wachsen damit über die anfängliche Klassifizierung der Ländergruppe (max. 4.999 US-Dollar) hinaus. Die zweite große Gruppe dieser Einkommensklasse wird von Entwicklungsländern wie Ägypten, Botswana, Mauritius etc. gebildet.

Tab. 3-7: Ergebnistableau (1.000 - 4.999 US-Dollar)

\begin{tabular}{ll|cc} 
& & $1998-2000$ & $2001-2003$ \\
\hline \multirow{2}{*}{ NIM } & Maximum der Gruppe & 0,0957 & 0,0680 \\
& Minimum der Gruppe & 0,0230 & 0,013 \\
& Durchschnittswert & 0,0565 & 0,0343 \\
\cline { 2 - 3 } & Differenz (Durchschnitt) & \multicolumn{2}{|c}{$-0,0222$} \\
& Differenz prozentual & \multicolumn{2}{|c}{$-39,3$ v.H. } \\
\hline \multirow{3}{*}{ PKE } & Maximum der Gruppe & 4.964 US-\$ & 4.770 US-\$ \\
& Minimum der Gruppe & 1.010 US-\$ & 918 US-\$ \\
& Durchschnittswert & 2.912 US-\$ & 3.830 US-\$ \\
\cline { 2 - 3 } & Differenz (Durchschnitt) & \multicolumn{2}{|c}{+918 US-\$ } \\
& Differenz prozentual & \multicolumn{2}{|c}{21,5 v.H. } \\
\hline \multirow{2}{*}{ Länder } & & \multicolumn{2}{|c}{21}
\end{tabular}

Die meisten Länder dieser Gruppe konnten ihr Pro-Kopf-Einkommen steigern, die Ausnahmen diesbezüglich bilden Bolivien, Chile und Ägypten. Die Nettozinsmarge ist in den betrachteten Zeiträumen von 0,0565 auf 0,0343 gesunken. Dies entspricht einem prozentualen Abbau von 39,3 v.H. Diese radikale Reduzierung der Nettozinsmargen im Durchschnitt liegt sogar höher als die Reduktion derselbigen bei den Industrienationen. Allerdings darf nicht übersehen werden, daß diese Reduzierung auf einem relativ hohen Niveau stattfindet. Während die 
Abb. 3-6: Vergleich der Zinsmargen (Einkommensklasse 1.000 - 4.999 USDollar)
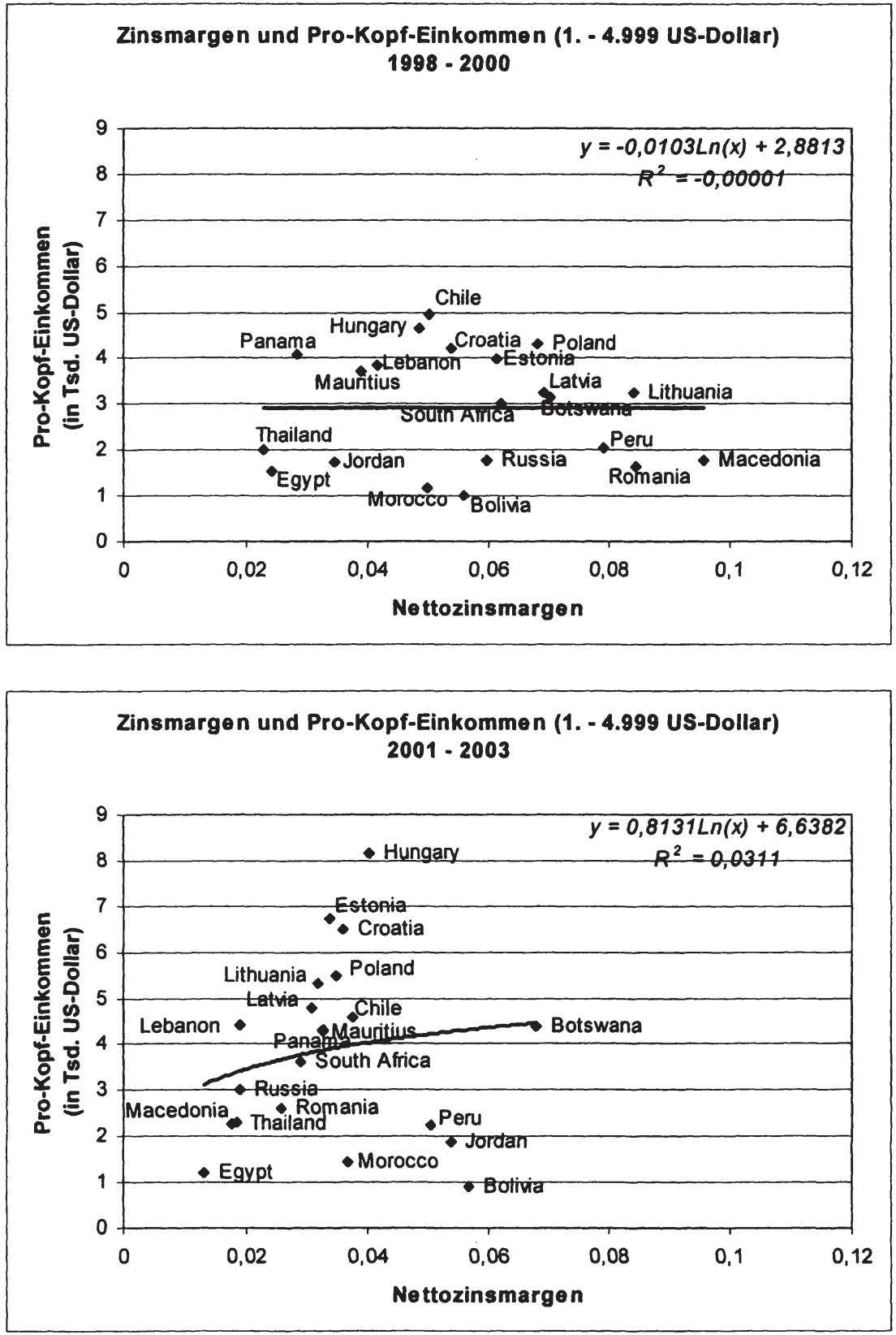
Industrienationen im Durchschnitt eine Senkung von 0,0266 auf 0,0179 vornahmen, sind die Zinsmargen in dieser Gruppe von 0,0565 auf 0,0343 gesunken. Nichtsdestotrotz läßt die starke Reduzierung darauf schließen, daß erhebliche Anstrengungen unternommen wurden, um die Finanz- und Bankenmärkte zu öffnen, wenn auch noch immer ein relativ hoher Protektionsgrad vorhält.

Die Reduktion der Zinsmargen ist auch graphisch sehr gut als kontraktive Bewegung zu erkennen. Zunächst liegt eine eher breite Streuung der Länder hinsichtlich der Nettozinsmargenwerte vor. Im zweiten Untersuchungszeitraum hingegen konzentriert sich die Verteilung mehrheitlich um die Nettozinsmargenwerte zwischen 0,03 und 0,04. Besonders beachtlich sind die hohen Einkommenszuwächse der osteuropäischen Staaten.

\subsubsection{Ländergruppe IV (Einkommensklasse $<1.000$ US-Dollar)}

Die letzte Einkommensklasse setzt sich aus den ärmsten Ländern der Welt zusammen, die über ein Pro-Kopf-Einkommen von weniger als 1.000 US-Dollar verfügen. Sie besteht aus afrikanischen, asiatischen und südamerikanischen Entwicklungsländern. Die Spanne der Pro-Kopf-Einkommen reicht von 991 (Honduras) bis 83 US-Dollar (Burundi).

Tab. 3-8: Ergebnistableau (< 1.000 US-Dollar)

\begin{tabular}{|c|c|c|c|}
\hline & & $1998-2000$ & $2001-2003$ \\
\hline & Maximum der Gruppe & 0,1161 & 0,1430 \\
\hline NIM & Minimum der Gruppe & 0,0348 & 0,0257 \\
\hline & Durchschnittswert & 0,0793 & 0,0658 \\
\hline & Differenz (Durchschnitt) & & \\
\hline & Differenz prozentual & & \\
\hline & Maximum der Gruppe & 991 US-\$ & 986 US-\$ \\
\hline PKE & Minimum der Gruppe & 100 US-\$ & 83 US-\$ \\
\hline & Durchschnittswert & 437 US-\$ & 502 US-\$ \\
\hline & Differenz (Durchschnitt) & & \\
\hline & Differenz prozentual & & \\
\hline Länd & & 9 & 9 \\
\hline
\end{tabular}


Abb. 3-7: Vergleich der Zinsmargen (Einkommensklasse $<1.000$ US-Dollar)
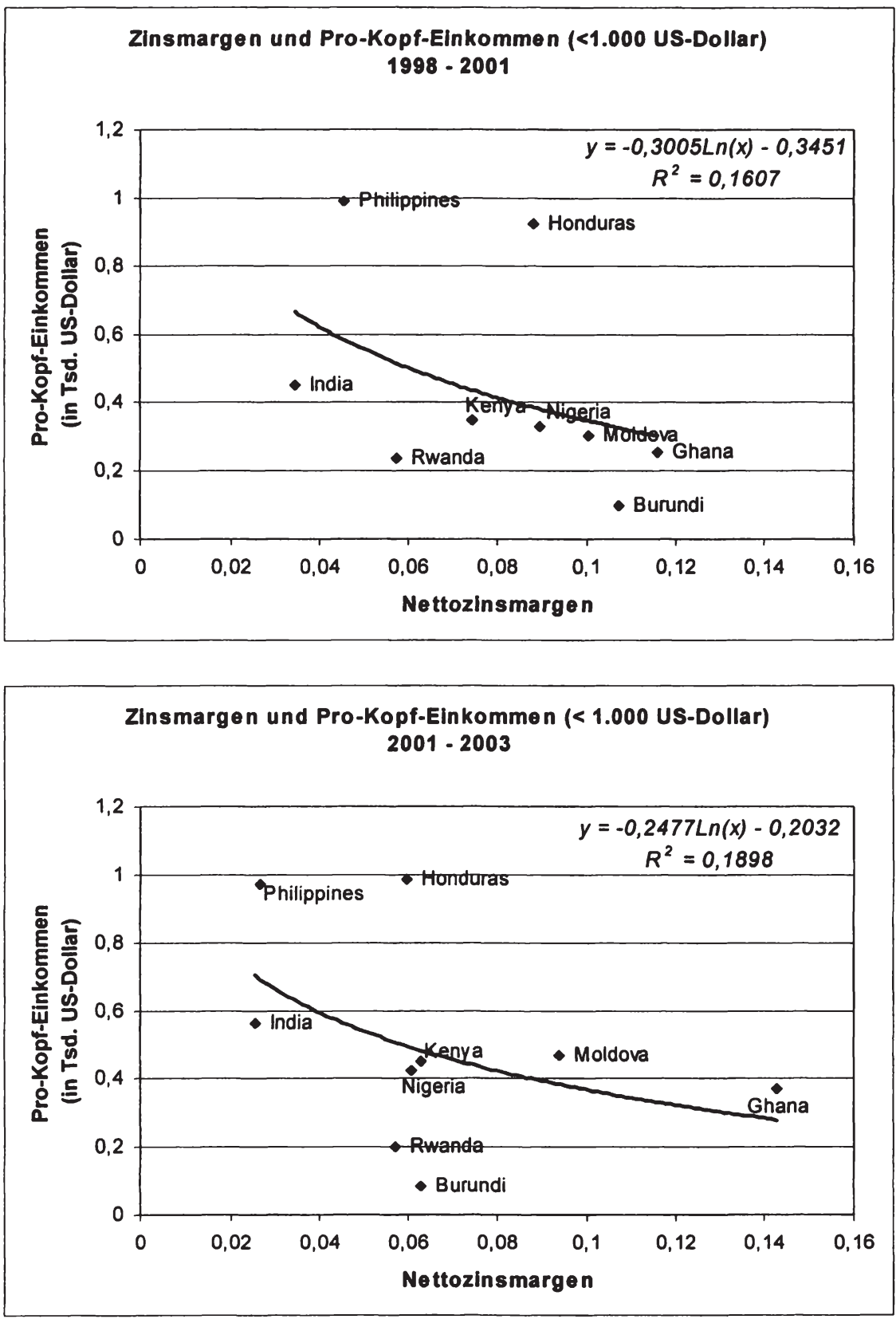
In dieser Gruppe erreichten nicht alle Länder eine Einkommenssteigerung: Burundi, die Philippinen und Ruanda mußten eine Verringerung des Pro-Kopf-Einkommens hinnehmen. Hinsichtlich der Entwicklung der Zinsmargen sind Indien und die Philippinen bemerkenswert: Im Vergleich zu den anderen Ländern dieser Gruppe weisen sie auffallend niedrige Zinsmargen auf, sie passen eher in die Ländergruppe III. Entsprechend hoch fällt die Spanne der Zinnmargen aus: Den höchsten Wert verzeichnet Ghana mit 0,1161 , der sogar noch auf 0,1430 steigt, den niedrigsten Indien mit 0,0257. Die meisten Länder dieser Gruppe halten eine sehr strikte Marktabschottung gegenüber dem Ausland aufrecht. Trotzdem sinken die Zinsmargen leicht. Aufgrund des starken Drucks zur Öffnung der Finanzmärkte, der im Rahmen der GATS-Verhandlungen ausgeübt wird, ist ein Trend zu einer weiteren Öffnung der Finanzmärkte und zu einer verstärkten Teilhabe am internationalen Kapitalverkehr zu erwarten. Auch graphisch ist keine klare Entwicklungsrichtung zu erkennen, die Nettozinsmargen haben sich nicht sehr verringert, auch wenn nun eine erhöhte Konzentration der Länder um den Wert von 0,06 feststellbar ist.

Um die unterschiedliche Entwicklung der Werte der einzelnen Ländergruppen zu verdeutlichen, werden die Durchschnittswerte der Nettozinsmargen und des ProKopf-Einkommens übersichtlich zusammengefügt.

Den Ländern der Einkommensklassen II und III gelang es mit Werten von 31,6 v.H. bzw. 31,5 v.H., die höchsten Zuwächse beim Pro-Kopf-Einkommen zu erreichen. Die Einkommenszuwächse in der Gruppe der ärmsten Länder (Gruppe IV) lagen prozentual hingegen nur halb so hoch mit 14,9 v.H. Hinsichtlich der Nettozinsmarge gelang der Einkommensklasse III die höchste prozentuale Absenkung, sie sank um beachtliche 39,3 v.H. Ähnlich hoch fielen die Reduzierung der Nettozinsmarge in der Gruppe I $(32,7$ v.H.) und noch vergleichbar hoch auch in der Gruppe II (25,67 v.H.) aus. Auch in dieser Kategorie erreichten die Entwicklungsländer der Gruppe IV mit einem Wert von 17 v.H. die geringste Reduzierung.

Allerdings relativiert die tabellarische Übersicht die Ergebnisse, da sie offenlegt, auf welchem Niveau die Veränderungen jeweils stattgefunden haben. So liegen die absoluten Werte der Nettozinsmargen der Einkommensklassen II bis IV deutlich über denen der Referenzgruppe I, also der Industrienationen und hoch entwickelten Länder. Die absolute Nettozinsmarge der Gruppe IV ist im Durchschnitt mehr als dreieinhalbfach höher als die der Industrieländer. ${ }^{1}$ Hier verbirgt sich also noch erhebliches Senkungspotential. Es soll aber nicht verhehlt werden, daß die Einkommen der Gruppe I mehr als 63 fach höher liegen als die der armen Länder, aus denen sich die Gruppe IV zusammensetzt.

Verwendet wurden fur diese Berechnung die Zahlen jungeren Datums, also des Zeitraumes 2001- 2003. 
\& Tab. 3-9: Ergebnisübersicht für alle Einkommensklassen

\begin{tabular}{|c|c|c|c|c|c|c|c|c|}
\hline \multirow{2}{*}{\multicolumn{2}{|c|}{ Einkommensklasse }} & \multirow{3}{*}{\begin{tabular}{|r|} 
Zeitraum \\
$1998-2000$
\end{tabular}} & \multicolumn{3}{|c|}{ Nettozinsmargen (NIM) } & \multicolumn{3}{|c|}{ Pro-Kopf-Einkommen (PKE) } \\
\hline & & & Durchschnitt & \begin{tabular}{|c|}
$\begin{array}{c}\text { Absolute } \\
\text { Verănderung }\end{array}$ \\
\end{tabular} & \begin{tabular}{|c|}
$\begin{array}{c}\text { Prozentuale } \\
\text { Verănderung }\end{array}$ \\
\end{tabular} & Durchschnitt & \begin{tabular}{|c|} 
Absolute \\
Verănderung
\end{tabular} & \begin{tabular}{|c|} 
Prozentuale \\
Verånderung
\end{tabular} \\
\hline \multirow{2}{*}{ I } & \multirow{2}{*}{$>15.000$ US-Dollar } & & 0,0266 & \multirow{2}{*}{$-0,0087$} & \multirow{2}{*}{$-32,7$ v.H. } & 25.941 US-\$ & \multirow{2}{*}{+6.028 US- $\$$} & \multirow{2}{*}{$+23,2$ v.H. } \\
\hline & & $2001-2003$ & 0,0179 & & & 31.969 US-\$ & & \\
\hline \multirow{2}{*}{ II } & \multirow{2}{*}{ 5. - 14.999 US-Dollar } & $1998-2000$ & 0,0335 & \multirow{2}{*}{$-0,0086$} & \multirow{2}{*}{$-25,67$ v.H. } & 10.232 US-\$ & \multirow{2}{*}{+3.234 US-S } & \multirow{2}{*}{$+31,6$ v.H. } \\
\hline & & $2001-2003$ & 0,0249 & & & 13.466 US-\$ & & \\
\hline \multirow{2}{*}{ III } & \multirow{2}{*}{ 1. -4.999 US-Dollar } & $1998-2000$ & 0,0565 & \multirow{2}{*}{$-0,0222$} & \multirow{2}{*}{$-39,3$ v.H. } & 2.912 US-\$ & \multirow{2}{*}{+918 US-\$ } & \multirow{2}{*}{$+31,5$ v.H. } \\
\hline & & $2001-2003$ & 0,0343 & & & 3.830 US-\$ & & \\
\hline \multirow{2}{*}{ IV } & \multirow{2}{*}{$<1.000$ US-Dollar } & $1998-2000$ & 0,0793 & \multirow{2}{*}{$-0,0135$} & \multirow{2}{*}{-17 v.H. } & 437 US-\$ & \multirow{2}{*}{+65 US-\$ } & \multirow{2}{*}{$+14,9$ v.H. } \\
\hline & & $2001-2003$ & 0,0658 & & & 502 US-\$ & & \\
\hline
\end{tabular}




\section{Determinanten der Zinsmargen - eine empirische Analyse}

\subsection{Daten und Methoden}

\subsubsection{Die Datenbasis}

\subsubsection{Quellen}

Die Daten für die Regressionen wurden zwei Erhebungen entnommen, die im Auftrag der Weltbank von dem Forscherteam Barth, Caprio und Levine durchgeführt wurden. Die Studien konzentrieren sich auf den Themenkomplex Bankenregulierung und Aufsichtsbehörden. Das Ziel der Untersuchung war der Nachweis von Zusammenhängen zwischen den Regulierungen und Kontrollpraktiken eines Landes einerseits und der Entwicklung und Leistungsfähigkeit des Bankensystems und letztlich auch Stabilität des Finanzsystems andererseits. Auf diesem Wege sollten die entscheidenden Faktoren zur Erreichung und Aufrechterhaltung einer adäquaten und potenten Finanzstruktur ermittelt werden. ${ }^{1}$ Die Datenerhebung umspannte für die erste Befragung den Zeitraum von Ende 1998 bis Anfang 2000. Die ersten Antworten gingen Ende des Jahres 1998 ein, der Großteil hingegen im Zeitraum von 1999 bis Februar 2000. Entsprechend wird dieser Untersuchungszeitraum in Zukunft unter der Bezeichnung 1998 - 2000 geführt. Eine Wiederholung der Studie erstreckte sich anschließend auf den Zeitraum von 2001 bis 2003. Die Daten sind seit März 2005 per Internet-Homepage zugänglich. ${ }^{2}$

Die Fragen der Erhebungen decken Bereiche wie die Regulierungen des Aktivitätsradius der Banken, die Verflechtungen von Banken mit anderen (Finanz-) Unternehmen, die Regulierungen der Marktzugänge für In- wie auch Ausländer, die Sicherung der Depositen, die Durchsetzungsmacht und Unabhängigkeit der kontrollierenden Behörden, die Klassifizierungssysteme für Anleihen, die Diversifikationsvorschriften etc. ab (Barth / Caprio / Levine 2002, 2). Die Anfragen wurden an die Zentralbanken und die mit der Banken- und Finanzmarktaufsicht betrauten Behörden der Länder gerichtet. Für die Kontaktaufnahme stellte das Basel Committee on Banking Supervision (BIS) Informationen und Adressen hinsichtlich relevanter nationaler Behörden und Ansprechpartner zur Verfügung. Auch die regelmäßig stattfinden Fortbildungsseminare der Weltbank für Schwellen- und Entwicklungsländer boten eine willkommene Plattform, um die Informationsträger um Auskunft zu bitten. Zum Teil wurden die Fragebögen persönlich von Mitarbeitern der Weltbank überbracht, die in Länder reisten, die den Fragebogen auf postalischem Wege nicht beantworteten. So konnte die Übergabe an die zuständigen Mitarbeiter sichergestellt werden.

Wie bei allen empirischen Erhebungen, die auf der Auskunft von Personen beruhen, ist die Korrektheit und Vollständigkeit der erhobenen Daten zu hinterfragen. Nicht alle kontaktierten Länder retournierten die Fragebögen, so gingen insgesamt im ersten Untersuchungszeitraum nur aus 107 Ländern Antworten ein. Auch gab 
es innerhalb eines Landes widersprüchliche Antworten von Beamten, die in unterschiedlichen Aufsichtsgremien tätig waren. Deshalb wurde die Authentizität der Antworten unter zu Hilfenahme von Berichten des Office of the Comptroller of the Currency (OCC) wie auch des Institute of International Bankers gegengeprüft. Bezüglich der Einlagensicherungssysteme konnten Informationen über die Financial Stability Forum's Working Group on Deposit Insurance geprüft werden.

Die Daten wurden in aggregierter Form für den Bankensektor eines Landes erhoben. ${ }^{1} \mathrm{Da}$ die Fragen sehr unterschiedliche Bereiche der Bankenmärkte und ihrer Überwachungssysteme betreffen, erfolgten die Antworten in

- , ,ja-nein“ - Form (bspw.: Existiert ein Einlagensicherungssystem?),

- konkreten Zahlen (bspw.: Wie hoch ist die Liquiditätsquote?) oder auch in

- Mehrfachausprägungen (bspw.: Sind den Banken Wertpapiergeschäfte erlaubt, nicht beschränkt, beschränkt oder verboten?).

Die Umsetzung der Informationen in für eine Schätzung verwertbare Zahlen ist dem Anhang Al (Legende der Variablen) zu entnehmen. Einige der Werte wurden als Durchschnitte ermittelt, um Zahlenausreißer zu relativieren (bspw. die Zinsmarge).

Der erste Datensatz umfaßt Angaben aus 107 Ländern für den Zeitraum von 1998 bis 2000. Für den zweiten Datensatz erfolgte die Befragung von 151 Ländern für die Periode 2001 bis 2003. Insgesamt liegen Daten für 166 Länder vor. ${ }^{2}$ Für 102 Länder liegen Daten sowohl aus der ersten wie auch aus der zweiten Untersuchung vor. Die Werte der Zinsmargen wurden nur in der zweiten Befragung ermittelt. Die fehlenden Zinsmargenwerte für die Befragung des Zeitraumes von 1998 bis 2000 wurden aus einer Untersuchung von Demirgüc-Kunt, Laeven und Levine (2003) ergänzt ${ }^{3}$, so daß die Daten beider Zeiträume untersucht werden können. $\mathrm{Zu}$ beachten bleibt, daß die Informationen nicht gleichmäßig über beide Zeiträume hinweg ermittelt wurden: $\mathrm{Zu}$ einem geringen Teil unterscheiden sich die Länder in den beiden Erhebungen. Auch weisen die Angaben einiger Länder partiell Lücken auf, so daß die folgenden Schätzungen in der Anzahl der Beobachtungen stark variieren können. Die Daten des Pro-Kopf-Einkommens der Länder (in US-Dollar) sind von der Weltbank erhoben worden. ${ }^{4}$ Für den Zeitraum 1998 bis 2000 wurden die Pro-Kopf-Einkommen des Jahres 2000 zugrundegelegt, für den Zeitraum 2001 bis 2003 die Pro-Kopf-Einkommen des Jahres 2003.

Die Fragebögen finden sich in den Anhängen A5 und A6.

Dies ist bedingt durch die Neuaufnahme oder den Austausch von Ländern in den Befragungen.

3 Die Zinsmargen fur Österreich, Australien und Dänemark wurden direkt bei den nationalen Zentralbanken erfragt.

4 World Bank, World Development Indicator, Quick Query, http://ddp-ext.worldbank.org. 


\subsubsection{Auswahl der Variablen: Kriterien}

Nicht alle ermittelten Informationen der Erhebung sind von Bedeutung für die zu untersuchende Fragestellung. Als relevante Daten für die Schätzung wurden diejenigen Informationen betrachtet, die Protektionspotentiale enthalten. Dies können Regulierungen sein, die bereits erheblich die Funktion der Banken beschränken und/oder in Zukunft für protektionistische Zwecke instrumentalisiert werden können. Folglich fanden Informationen, die wahrscheinlich nicht Determinanten der Zinsmargen sind und kein Protektionspotential bergen, auch keinen Eingang in die Regression.

Einige Informationen standen in schlecht verwertbarer Form zur Verfügung und flossen nicht in die Regressionen ein. So wurde bspw. die Frage nach dem Mindestkapital bei einem Bankenmarkteintritt sowohl in US-Dollar wie auch in lokaler Währung beantwortet. Bei Staatsverbänden, die unter einer Zentralbank zusammengefaßt werden, erfolgte die Bereitstellung der Daten aggregiert für alle beteiligten Länder (beispielsweise die Central Bank of West African States oder die Eastern Caribbean Central Bank). ${ }^{\prime}$

Auch die Vollständigkeit der Datensätze spielte eine große Rolle bei der Entscheidung für oder gegen die Verwendung einer Variablen. Lagen nur sehr wenige Antworten zu einer Frage vor, so wurde auf eine Berücksichtigung dieser Daten in der Regression verzichtet. Die Fragebögen für die zweite Periode ermittelten zusätzliche Informationen, die für den ersten Untersuchungszeitraum nicht vorliegen. Diese Daten standen somit nicht für einen direkten Vergleich zur Verfügung. Im Hinblick auf die zu untersuchenden Fragestellungen wären Aussagen, die sich spezifisch auf den Umgang der Länder mit ausländischen Banken beziehen, wünschenswert gewesen, sie liegen aber in derart expliziter Form leider nicht vor.

Ein generelles Problem derartiger Erhebungen stellt die mangelnde Möglichkeit zur Verifizierung der Daten dar. Nicht auszuschließen ist, daß einige Daten (unabsichtlich oder absichtlich) verfälscht wurden.

\subsubsection{Die Methodologie}

Aus Gründen der Übersichtlichkeit werden die zur Verfügung stehenden Informationen thematisch zu Variablengruppen zusammengestellt. Um den Einfluß von Marktzugangsbedingungen, der Ausgestaltung der institutionellen Rahmenbedingungen und auch der Protektion auf die Zinsmargen zu schätzen, wird unter Verwendung der Kontrollvariablen Pro-Kopf-Einkommen (GDP per capita) und

1 So lautet die Antwort der westafrikanischen Staaten auf die Frage: „How many professional bank supervisors are there in total?"

„40 for all countries belonging to the Central Bank of West African States.” 
Kapitalquote folgende (OLS-) Regressionsgleichung für den Querschnitt geschätzt:

$$
N I M_{i}=\alpha+\beta_{1}^{*} P K E_{1}+\beta_{2}^{*} K \operatorname{Kap}_{i}+\beta_{3} * \text { Variable }_{i}+\ldots \ldots+\beta_{z}^{*} \text { Variable }_{i}+u_{i}
$$

mit:

$\begin{array}{ll}\alpha & =\text { konstanter Parameter, } \\ \beta_{1}, \ldots, \beta_{z} & =\text { zu schätzende Parameter (Regressionskoeffizienten), } \\ N I M_{i} & =\text { Zinsmarge des Landes } i \\ P K E_{i} & =\text { Pro-Kopf-Einkommen des Landes } i \\ \text { Kap }_{i} & =\text { Kapitalquote } \\ \text { Variable } A_{i}, \ldots, Z_{i} & =\text { die getesteten Variablen der Erhebung, } \\ u_{i} & =\text { Störgröße des Landes } i .\end{array}$

In beiden Zeiträumen hat das Pro-Kopf-Einkommen einen signifikanten Einfluß auf die Zinsmargen. Diese Kontrollvariable wird fortan in jeder Schätzung verwendet. Das Pro-Kopf-Einkommen geht mit einem negativen Koeffizienten in die Schätzung ein. Das heißt: Je höher das Pro-Kopf-Einkommen eines Landes, um so geringer ist die Zinsmarge. Hohe Pro-Kopf-Einkommen zeichnen Länder aus, die sich tendenziell durch einen hohen Entwicklungsstand auszeichnen. Diese Länder verfügen in der Regel über ein leistungsfähiges und sich durch einen hohen Wettbewerbsgrad auszeichnendes Bankensystem, was eine niedrige Zinsmarge impliziert. $^{2}$

Als ebenfalls guter Schätzer erwies sich die Kapitalquote (Variable d34 bzw. d342). Sie geht mit einem positiven Vorzeichen in die Schätzgleichung ein: Je höher die von den Banken gehaltenen Kapitalreserven in einem Land, desto höher ist tendenziell die Zinsmarge. Die Kapitalquoten repräsentieren Reserven für unvorhersehbare Ereignisse, es handelt sich um Rücklagen der Banken für Liquiditätsnotfälle. Hohe Kapitalquoten signalisieren somit, daß in einem Land ein hohes $\mathrm{Maß}$ an Unsicherheit vorliegt. Je größer die Unsicherheit, um so höher die Kapitalquote und um so höher ist die Zinsmarge eines Landes.

Gemäß der obigen Regressionsgleichung werden, neben den Kontrollvariablen des Pro-Kopf-Einkommens und der Kapitalquoten, einzelne Regressionen der Variablengruppen vorgenommen. Diese lauten:

Die Kapitalquote ist definiert als Anteil des Eigenkapitals am Gesamtvermøgen einer Bank.

Vgl. Kapitel 3.3.1 Gesamtentwicklung der Zinsmargen und insbesondere Kapitel 3.3.2 Ländergruppe I (Einkommensklasse $>15.000$ US-Dollar). 
- Weitere Kapitalquoten,

- Attraktivität des Bankenmarktes (Gewinnpotential),

- Eigenkapital der Banken,

- Verflechtungsgrad,

- Konzentrationsgrad,

- Geschäftsfelder der Banken,

- Rechnungslegung / Rechnungsprüfung,

- Liquidität (im weiteren Sinne),

- Einlagensicherungssysteme,

- Offenlegungspflichten / öffentliches Monitoring,

- Haftbarkeit bzw. juristische Verantwortung des Bankenmanagements,

- Kontrollintensität,

- Economic Freedom Indicator,

- Rechte der Aufsichtsbehörden,

- Ratings,

- Anzahl der geschlossenen oder fusionierten Banken,

- Staatliche und ausländische Anteile am Bankenmarkt,

- Befugnisse der Aufsichtsbehörde.

Im ersten Schritt wird die Zinsmarge nur auf die Variablen einer Gruppe regressiert, bspw. alle verfügbaren Informationen der Gruppe „Kapitalquoten“ (Teilregression). Anschließend müssen die einflußreichen von den nicht determinierenden Variablen unterschieden werden. Als Auswahlkriterium für die Bereinigung der Teilschätzungen dienen die Ablehnungswahrscheinlichkeit einer Variablen (Signifikanzniveau) und ihr t-Wert. Interaktionseffekte zwischen den thematischen Gruppen werden zunächst vernachlässigt und erst in der abschließenden Gesamtschätzung berücksichtigt. Dieselbe Vorgehensweise wird für die Daten der späteren Untersuchungsperiode verwendet. So lassen sich Veränderungen der Signifikanz der Determinanten direkt ermitteln.

Nachdem durch die Teilschätzung die einflußreichen Determinanten der Zinsmargen isoliert wurden, erfolgte die Ermittlung der besten Schätzgleichungen für die beiden vorliegenden Zeiträume. Die Kombination der Variablen verschiedener Themenbereiche in einer Schätzung deckte Überlagerungen in ihrer Wirkung auf die Nettozinsmarge auf.' In einem weiteren Schritt wurden die insignifikanten

1 Vgl. hierzu Kapitel 4.2.1.12 Kontrollintensităt. 
Variablen ausgeschlossen. Da einige Determinanten nur in Kombination mit bestimmten Variablen insignifikant wurden, waren viele einzelne Regressionen nötig, um ihr Verhalten im Kontext verschiedener Gruppierungen zu ermitteln. Diese Vorgehensweise erfordert den häufigen Rückgriff auf zunächst aus der Regression entfernte Variablen und die erneute Schätzung.

Um sicherzustellen, daß keine Variable zu unrecht ausgeschlossen wurde, wurden auch die in dem ersten Prüfungsschritt (Teilregressionen) ausgeschlossenen Variablen in der Gesamtregression erneut auf ihre Bedeutung für die Determinierung der Zinsmargen überprüft. Eine gewisse Pfadabhängigkeit in der Reihenfolge der Auswahl der Variablen ist nicht auszuschließen. Dieses Risiko wurde durch die wechselnde Reihenfolge in der Einbindung der Variablen in die Regressionen und zahlreiche Wiederholungen minimiert. Letztlich schälten sich durch dieses Verfahren die einflußreichsten Determinanten der Nettozinsmarge heraus.

Bei der Ermittlung der aussagekräftigsten Schätzgleichungen für die beiden Untersuchungszeiträume wurde anschließend eine robuste Schätzung durchgeführt, um für auftretende Heteroskedastizität zu korrigieren. Damit wird geprüft, ob die Varianzen der Störgrößen in der Stichprobe konstant sind (robuste Schätzer).

Zur tabellarischen Darstellung der Ergebnisse sei angemerkt, daß der besseren Übersicht halber die Schätzungen für beide Zeiträume nebeneinander angeordnet werden. Zunächst wird eine Schätzung mit allen Variablen der thematischen Gruppe für die erste Periode vorgenommen. Anschließend wird die um die nicht signifikanten Variablen bereinigte Schätzung dargestellt. Diese Vorgehensweise wird anschließend für die Daten der zweiten Periode wiederholt. Die Tabellen führen die Koeffizienten samt ihrer Vorzeichen auf. Die dazugehörigen $t$-Werte sind in Klammern beigefügt. Das Signifikanzniveau wird anhand des üblichen Symbols „Stern“ verdeutlicht. Drei Sterne entsprechen einem Signifikanzniveau von einem Prozent, zwei Sterne von fünf Prozent und ein Stern markiert die 10prozentige Signifikanz. Im Schlußteil der Tabelle befinden sich Angaben zur Anzahl der Beobachtungen bzw. Länder, von denen verwertbare Informationen vorliegen. Diese variieren je nach Frage zum Teil sehr stark, da die Datenerhebung Lücken aufweist.

Für jede Schätzung werden die Bestimmtheitsmaße $R^{2}$ und adjustiertes $R^{2}$ angegeben. Sie erklären die Varianz der Nettozinsmarge. Besondere Beachtung findet das adjustierte $R^{2}$. Es ist von der Anzahl der einbezogenen Variablen weitgehend unabhängig und für den Vergleich des Erklärungsvermögens dem einfachen $R^{2}$ vorzuziehen (Hackl 2005, 76). Ein hoher F-Wert führt zur Ablehnung der Nullhypothese, er bestätigt also die Relevanz der Schätzung (F-Test).

Die Schätzungen wurden unter Zuhilfenahme des Programms STATA $^{\circ}$ Statistics / Data Analysis, Version 8.2 vollzogen. 


\subsubsection{Variablengruppen und ihre Protektionspotentiale}

Die zur Verfügung stehenden Informationen werden zu Variablengruppen gebündelt und anschließend darauf getestet, inwieweit sie die Zinsmargen determinieren. Zunächst werden jedoch Hypothesen im Hinblick auf ihr Protektionspotential gebildet. Analysiert wird, worin es konkret besteht, wie es genutzt werden kann und welche Folgen zu erwarten sind.

\section{Kapitalquote}

Die Kapitalquote repräsentiert den Eigenkapitalanteil am Gesamtvermögen einer Bank. Ist die Haltung von hohen Kapitalquoten gesetzlich fixiert, so wird dadurch vergleichsweise viel Kapital der Kreditinstitute gebunden. Diese Finanzmittel stehen nicht für die Kreditvergabe zur Verfügung. In der Tendenz reduzieren hohe Kapitalquoten also das Kreditvergabevolumen. Im Regelfall sollte dieselbe Eigenkapitalquote für alle in einem Land operierenden Kreditinstitute Anwendung finden. Die Eigenkapitalquote kann protektionistisch eingesetzt werden, wenn sie diskriminierend gegenüber ausländischen Banken verwendet wird. Wird ausländischen Banken auferlegt, einen höheren Eigenkapitalanteil zu halten, behindert dies ihre Kreditvergabe, ihre Eigenkapitalrendite sinkt tendenziell. Inländischen Banken erhalten somit einen Wettbewerbsvorteil.

\section{Attraktivität des Bankenmarktes (Gewinnpotential)}

Die Anzahl der Bewerbungen um Erteilung einer Banklizenz aus dem In- und Ausland ist eine Maßzahl für die Attraktivität eines Bankenmarktes. Hohe Bewerberzahlen sind insbesondere bei Bankenmärkten zu erwarten, die über längere Zeiträume hinweg abgeschottet waren. Dadurch konnte sich dort eine inländische Bankenlandschaft etablieren, die aufgrund mangelnder Konkurrenz von außen oligopolistische Marktstrukturen aufweist. Der Mangel an Konkurrenz begünstigt die Banken gegenüber den weiteren Marktteilnehmern (Unternehmen und Haushalte). Verglichen mit einer Situation unter vollkommener Konkurrenz setzen die Banken geringere Dienstleistungsmengen zu höheren Preisen um. Dadurch erzielen sie relativ hohe Gewinne. Diese überdurchschnittlich hohen Gewinne verleiten die ausländische, expansiv orientierte Konkurrenz zu einem Engagement auf diesen Märkten, um an ihnen zu partizipieren. Die Ablehnungsquote ausländischer Bewerbungen ist ein eindeutiges Indiz für die Existenz von Protektion. Die vollständige Abschottung des Marktes wird durch die Ablehnung aller Bewerbungen von ausländischen Banken um Lizenzen erreicht. Mit diesem Instrument können die nationalen Behörden direkt ins Marktgefüge eingreifen. Die verantwortlichen Akteure sind in der Lage, die Anzahl der Marktteilnehmer festzulegen und damit auch den vorherrschenden Wettbewerbsgrad. Alle Gewinnmöglichkeiten bleiben inländischen Banken vorbehalten. Die negativen Folgen sind vergleichsweise geringe Kreditvergabevolumina zu relativ hohe Zinsen. Damit wird das Entwicklungspotential der betroffenen Volkswirtschaft beschränkt. Auf betriebswirtschaftlicher Ebene ist zu erwarten, daß die inländischen Banken international 
nicht konkurrenzfähig sind und ineffiziente Strukturen aufweisen. Damit ist ihr Aktionsradius auf den geschützten inländischen Markt beschränkt. Sie sind nicht in der Lage, expansive Strategien umzusetzen und ausländische Märkte zu erobern. Ihre Entwicklungschancen sind folglich als gering einzuschätzen, sie benötigen den Schutz des abgeschotteten Marktes.

\section{Eigenkapital der Banken (Konzentration/Verflechtung)}

Einige Länder weisen Beschränkungen hinsichtlich des Eigenkapitals im Besitz einer Person bzw. im Besitzstand verwandter Personen oder Gesellschaften auf. Insbesondere Entwicklungs- und Schwellenländer verfügen oftmals nur über eine sehr beschränkte Anzahl von Kreditinstituten. Die Konzentration des Bankenbesitzes in den Händen einiger weniger Personen oder Familien sichert diesem Personenkreis annähernd unbegrenzte Einflußmöglichkeiten. Sie verfügen über ein immenses Gewicht, da sie durch ihr Kreditvergabeverhalten massiv in die wirtschaftspolitische Entwicklung eingreifen und $\mathrm{zu}$ bedeutenden Machtfaktoren werden. Regelungen, die eine Akkumulation des wirtschaftlichen Einflusses und die Machtkonzentration in der Hand einiger weniger Personen oder Familien beschränken, sind deshalb aus wettbewerbspolitischer Sicht tendenziell als förderlich und positiv für die weitere Entwicklung eines Landes zu bewerten. Unter diesem Aspekt sollte sich die Beschränkung des Eigenkapitalbesitzes positiv auf die Zinsmarge als Indikator der Protektion auswirken. Hinsichtlich des Engagements ausländischer Interessenten ist die Lage aber anders zu beurteilen: Ausländern wäre ebenso der alleinige Besitz einer Bank verwehrt wie Inländern. Somit muß ein ausländischer Investor sich stets um inländische Teilhaber bemühen, da er allein nicht legal in den Besitz einer Bank gelangen kann. Regulierungen dieser Art sind sehr gut geeignet, um ausländische Investoren vom Markteintritt abzuhalten. Informelle Kartelle und gegenseitige Kreuzbeteiligungen der führenden Bankenfamilien können deren Marktpositionen untereinander absichern. Neuen Konkurrenten kann so der Marktzutritt verwehrt werden.

Zielte diese Regulierung ursprünglich auf die Förderung des Wettbewerbs, so ist sie nur allzu leicht zu umgehen. Sie ist damit ein Beispiel für ,gut gemeint, aber schlecht gemacht“. Zielte sie jedoch auf den Ausbau von Marktzugangsbarrieren, so ist sie geschickt gewählt. Ausländischen Investoren stehen zumeist nicht die sozialen Kontakte und informellen Netzwerke zur Verfügung, um sich friktionslos in dieses Beziehungsnetz $\mathrm{zu}$ integrieren. Interessant wären explizite Daten bezüglich der Beschränkung des Eigenkapitalbesitzes in ausländischer Hand, die aber in dieser Untersuchung leider nicht vorliegen.

\section{Verflechtungsgrad zwischen Banken und Finanzunternehmen}

Ein hoher Grad der Verflechtung zwischen Banken und weiteren, im Finanzsektor tätigen Unternehmen (Versicherungen, Finanzdienstleister etc.) kann ebenfalls Einfluß auf die Zinsmargen nehmen. Ein hoher Verflechtungsgrad bietet den Beteiligten die Möglichkeit, sich gemeinsam gegen ausländische Konkurrenz zu 
positionieren. Sie können ausländischen Anbietern den Markteintritt erheblich erschweren. Die Ökonomie hat diesbezüglich zwei miteinander konkurrierende Betrachtungsweisen entwickelt: die Efficient Structure Theory und das Konzept der Marktmacht.

Eine starke Verflechtung von Banken und anderen Finanzunternehmen kann positive Folgen durch Synergien aufweisen (Kombination von Bank- und Versicherungsdienstleistungen o.ä.), die eine kostengünstige, individuell an den Kunden angepaßte Dienstleistungserstellung ermöglichen. Diese hohe Effizienz in der Produktion von Dienstleistungen kann nur durch die Verknüpfung der daran beteiligten Unternehmen erreicht werden (Efficient Structure Theory). Die hohe Marktkonzentration wirkt sich somit nicht negativ aus. Das Konzept der Marktmacht hingegen sieht in der marktbeherrschenden Stellung weniger, miteinander verbundener Finanzunternehmen den Hinweis auf einen Mangel an Wettbewerb, der zur Rentenabschöpfung genutzt wird. In diesem Fall wird die stark ausgeprägte Marktmacht zur eigenen Gewinnmaximierung und zulasten Dritter, der Kunden, genutzt. Die Folgen wären hohe Preise, die für Finanzaktiva und -dienstleistungen entrichtet werden müssen, wie auch ein suboptimales Ausmaß an Kapitalangebot und -nachfrage. Die Frage, welche Theorie die Realität eher widerspiegelt, muß die Empirie klären.

Charakterisiert die Efficient Structure Theory den Bankenmarkt, so dient die Aufrechterhaltung eines hohen Verflechtungsgrades inländischer Finanzbranchen nicht protektionistischen Zielen. Liegt allerdings eine Konzentration der Marktmacht vor, so ist die Situation anders zu bewerten. Der Verzicht auf die Zerschlagung dieser mächtigen Konglomerate dient sehr wahrscheinlich der Aufrechterhaltung der marktbeherrschenden Position inländischer Unternehmen. Staatliche Behörden können durch Zutrittsbeschränkungen o.ä. versuchen, den Marktzugang ausländischer Wettbewerber zu beschränken. Somit beinhalten auch diese Variablen ein gewisses $\mathrm{Ma} ß$ an Protektionspotential.

\section{Konzentrationsgrad}

Thematisch eng verbunden mit der Frage nach dem Verflechtungsgrad sind Angaben bezüglich des Konzentrationsgrades der Märkte. Auch hier stehen sich die zwei oben skizzierten Lehrmeinungen Efficient Structure Theory und das Konzept der Marktmacht/ Monopolisierung gegenüber. Das Indiz des hohen Konzentrationsgrads einer Branche erlaubt keinerlei Rückschlüsse darauf, ob dieser gezielt aufgrund protektionistischer Absichten herbeigeführt wurde. Tendenziell liegt die Vermutung nahe, daß eine hohe Marktkonzentration die Folge einer zuvor eher protektionistisch geprägten Politik der Entscheidungsträger ist - falls es sich bei den marktmächtigen Unternehmen um inländische Banken handelt. Diese Variablen beinhalten kein Protektionspotential, wenn der Markt bestreitbar ist und ausländische Konkurrenzunternehmen zugelassen werden. Wie oben bereits ausgeführt, kann eine hohe Marktkonzentration auch der Effizienz geschuldet sein und damit der Realisierung des optimalen Marktergebnisses dienen. 


\section{Geschäftsfelder der Banken}

Banken konzentrieren sich in der Regel nicht allein auf die Kreditvergabe. So ist die Erweiterung ihrer Geschäftstätigkeit um Aktivitäten in den Bereichen Wertpapiere, Versicherungen und/oder Immobilien üblich. Ein breites Betätigungsfeld ermöglicht das Angebot von Kuppelprodukten, die Risikostreuung wie auch die Erschließung neuer Gewinnquellen. Zyklische Gewinnschwankungen eines Geschäftsbereiches lassen sich durch die Forcierung anderer Bereiche kompensieren. Verluste im Kreditgeschäft können so durch Gewinne im Wertpapierhandel aufgefangen werden. Die Erschließung alternativer Einnahmen fördert die Stabilität des Bankensektors und erleichtert so die Überwindung von Kreditkrisen. Wird nur den ausländischen Banken die Begrenzung ihrer Aktivitäten auf die Kreditvergabe auferlegt, so liegt eindeutig protektionistisches Verhalten vor. ${ }^{1}$ Die ausländischen Banken werden benachteiligt, da die weiteren Geschäftsfelder mit ihren Gewinnpotentialen den einheimischen Kreditinstituten vorbehalten bleiben. Abgesehen von den entgangenen Gewinnen sind ausländische Banken damit auch lokalen Krisen des Kreditsektors weitaus stärker unterworfen.

\section{Rechnungslegung}

Vorschriften, die eine korrekte Bilanzierung sicherstellen, wirken sich im sensiblen Finanzsektor positiv auf das Vertrauen der Einleger und Investoren aus. Regulierungen, die eine realistische, bilanzielle Abbildung der Banken durchsetzen können, dienen der Aufrechterhaltung der Finanzstabilität, da die Insolvenz einer Bank sich schnell zu einer Finanzmarktkrise ausweiten kann. Ein Mindest$\mathrm{ma} ß$ an Kontrolle seitens der Aufsichtsbehörden ist somit unerläßlich für die Aufrechterhaltung der Funktionstüchtigkeit des Finanzsystems. Da die Regeln zur Rechnungslegung und Bilanzierung gleichermaßen für in- und ausländische Unternehmen gelten, weisen sie kein Protektionspotential im herkömmlichen Sinne auf. Von Interesse ist aber, ob sich die Intensität der Kontrollen auf dem Finanzmarkt widerspiegelt und in der Höhe der Zinsmargen abbildet. Dieser Index dient auch als Effizienzmaß des Bankenmarktes. Bei Relevanz dieser Variablen ist eine weitere Gruppe von Determinanten der Zinsmargen identifiziert.

\section{Liquidität (im weiteren Sinne)}

Die gesetzliche Verpflichtung der Banken, liquide Mittel in einer vorgeschriebenen Mindesthöhe zu halten, dient der Überbrückung kurzfristiger Finanzierungsengpässe. Gleichzeitig werden finanzielle Mittel gebunden, die keiner gewinnbringenden Investition zugeführt werden können. Je detaillierter die Anforderungen an die Höhe sowie die Zusammensetzung der zu haltenden liquiden Mittel sind, um so geringer fällt der Gestaltungsspielraum der Banken aus. Diese Einschränkung der Dispositionsmöglichkeiten der Banken wirkt sich tendenziell ko-

In Malaysia wird ausländischen Banken die Kreditvergabe nur in einer exakt abgegrenzten Branche erlaubt. 
stenerhöhend aus. Folglich sollten Länder mit stark reglementierenden Vorschriften auch höhere Vorhaltekosten aufweisen. Da die Liquiditätsvorschriften gleichermaßen für die in- und ausländischen Kreditinstitute gelten, ist hier kein Protektionspotential vorhanden - es sei denn, daß die Beschaffung im Ausland bzw. das Halten liquider Mittel in ausländischen Währungen oder Wertpapieren verboten wird. Derartige Bestimmungen dienen der Marktabschottung, die Finanzaktiva sollen nach Möglichkeit nicht das Inland verlassen und die Refinanzierung der Banken allein über die nationale Zentralbank bzw. das inländische Finanzsystem erfolgen. Damit wird der freie Kapitalverkehr unterbunden; es kann kein inländisches Kapital abfließen.

\section{Einlagensicherungssysteme}

Ein Einlagesicherungssystem fungiert als Versicherung der Depositen gegen einen eventuellen Verlust. Im Insolvenzfall einer Bank erhalten die Kunden (zumindest zum Teil) ihre Einlagen zurück. Der zumeist gesetzlich geregelte Schutz der Depositen stärkt somit das Vertrauen der Kunden in das Finanzsystem. Ein hohes $\mathrm{Maß}$ an Vertrauen ist unabkömmlich für die Bereitstellung von Finanzmitteln durch die Haushalte, die von den Banken für die Kreditvergabe benötigt werden. Protektionsrelevant ist nur die Durchsetzung diskriminierender Regelungen im Einlagensicherungssysteme. Mit Verweis auf die Tatsache, daß die nationalen Zugriffsmöglichkeiten auf ausländische Banken erheblich eingeschränkt sind, können diesen höhere Prämien abverlangt werden. Damit würden ausländische Banken bei der Aufbringung von Finanzmitteln zusätzlich belastet. Ihre „Produktionskosten" sind höher als die vergleichbarer inländischer Kreditinstitute. Sie wären im Wettbewerb benachteiligt. Von Interesse ist in dieser Variablengruppe also nicht nur die Frage nach dem Einfluß auf die Zinsmarge, sondern auch nach dem Protektionsgehalt. Falls diese Variablen Determinanten der Zinsmargen sind, so würde gemessen, wie stark das Vertrauen der Marktteilnehmer und die Sicherheit der Geldanlagen in das Handlungskalkül der Marktteilnehmer eingeht. Die Reduktion der Unsicherheit durch ein funktionierendes Einlagensicherungssystem würde sich folglich an relativ niedrigen Zinsmargen ablesen lassen. Entsprechend werden Aspekte überprüft wie die Existenz eines Einlagensicherungssystems, die Finanzierung der Einlagensicherung, ob das System von staatlicher, privatwirtschaftlicher Seite oder beiden gemeinsam getragen wird, aber auch Erfahrungen mit Krisen in der Vergangenheit (Erfolgte beim letzten Bankzusammenbruch eine vollständige Kompensation der Einleger?).

\section{Offenlegungspflichten}

Die Beobachtung der Finanzmärkte und ihrer Akteure beschränkt sich mittlerweile nicht mehr auf nationale Behörden. Das Monitoring durch eine breite Öffentlichkeit (institutionelle und private Anleger, Rating-Agenturen, die Presse etc.) hat erheblich an Bedeutung gewonnen. Diese nicht-staatlichen Beobachter haben sich als ein wirksames Kontrollinstrument erwiesen. Gerade die institutionellen Anleger und Rating-Agenturen sind in der Lage, Fehlverhalten von Banken abzustra- 
fen - und zwar nicht juristisch, sondern weitaus wirkungsvoller durch finanzielle Konsequenzen. Damit können sie den Banken und Finanzintermediären eine größere Transparenz abverlangen. In diesem Bereich ergibt sich für die staatlichen Akteure kein Protektionspotential. Einzig unter dem Aspekt der Verringerung von Unsicherheiten auf dem Finanzmarkt hinsichtlich zukünftiger Entwicklungen oder wahrheitsgemäßer Berichte ist dieser Komplex an Variablen von Bedeutung. Protektionspotential ist nicht vorhanden.

\section{Haftbarkeit bzw. juristische Belangbarkeit des Bankmanagements}

Die juristischen Möglichkeiten, das Management einer Bank für ihr Fehlverhalten zur Verantwortung zu ziehen, werden nun untersucht. Weist ein Land eine nur schwach entwickelte Rechtsordnung auf, mit einer gering ausgeprägten Neigung zur Verfolgung von Wirtschaftskriminalität oder aber vergleichsweise milder Bestrafung derartiger Vergehen, so liegt auch eine vergleichsweise geringe Entdekkungs- und Bestrafungswahrscheinlichkeit vor. Diesen Umstand kann das Bankmanagement für sich nutzen, es wird tendenziell eher risikoreiche Anlagestrategie verfolgen, da es für eventuelles Fehlverhalten wie Außerachtlassung der Sorgfaltspflicht nicht oder zumindest nicht schwer bestraft werden kann. Aus Mangel an Angst vor einer rigorosen Bestrafung kann es sich sogar dazu ermutigt sehen, sich betrügerisch zu verhalten. Die persönlichen Folgen wären im Falle einer Entdeckung nicht allzu gravierend und die Gewinnpotentiale sind hoch. Entstandene Schäden könnten nicht den Managern angelastet werden, sondern wären von den Eigentümern und Kunden der Bank zu tragen. Der Konkurs einer oder mehrerer Banken strahlt aber auch auf das Finanzsystem aus. Ein erhobener Betrugsverdacht oder eine unseriöse, gescheiterte Anlagestrategie löst erhebliche Verunsicherungen bei den Kapitalmarktteilnehmern aus und kann zu panikartigen Reaktionen führen. Instabilitäten oder Zusammenbrüche der Finanzmärkte werden, wegen ihrer schwer abschätzbaren und in der Regel desaströsen Folgen für die gesamte Volkswirtschaft, zu verhindern gesucht. Entsprechend müssen den nationalen Aufsichtsbehörden juristische Zugriffsrechte eingeräumt werden. Bei einer Auswertung unter protektionistischen Gesichtspunkten ist auch in dieser Variablengruppe das diskriminierende Verhalten gegenüber ausländischen Banken anzuführen bspw. in Form von weitreichenden Zugriffsrechten oder intensiveren Kontrollen. Hierfür liegen aber keine Hinweise vor.

\section{Kontrollintensität}

Effektive und qualitativ hochwertige Kontrollen senken das Risiko betrügerisch agierender Banken und minimieren die Verluste der Bankkunden. Damit wird auch das Vertrauen der Bankkunden in die Finanzinstitutionen gestärkt. Innerhalb dieser Variablengruppe werden unterschiedliche Aspekte der Bankenkontrolle untersucht. Nicht nur die generelle Anzahl der mit der Bankenüberwachung betrauten Mitarbeiter findet Berücksichtigung, sondern auch deren Erfahrungsgrad, deren Verhaltensweisen sowie die Anzahl der Kontrollen, die regelmäßig stattzufinden haben. Da die Überprüfungen der Banken von den Aufsichtsbehörden z.T. 
nach eigenem Ermessen erfolgt, kann hier ein diskriminierendes Verhalten der verantwortlichen Beamten leicht zum Tragen kommen. Für ein solches Verhalten gibt es keine konkreten Belege, aber immer wieder Hinweise (Feketekuty 1988). Durch diskriminierend agierende Aufsichtsbehörden können ausländische Konkurrenten erheblich geschädigt und in ihrer Geschäftstätigkeit behindert werden. Dieser inoffizielle Bereich liefert einer protektionistisch agierenden Wirtschaftspolitik reichlich Ansätze, um ihre Ziele zu verfolgen. Erfüllen inländische Banken die Auflagen nicht, so kann eher einmal wohlwollend darüber hinweggeblickt werden, während derartige Verfehlungen bei ausländischen Banken strikt geahndet werden. Hierbei handelt es sich nicht um quantifizierbare Vorteile, aber um Vorteile, die diskriminierend gewährt werden können.

\section{Economic Freedom Indicator}

Zur Erfassung des allgemeinen wirtschaftlichen Klimas, des Grades gewährter ökonomischer Freiheiten wird der Economic Freedom Indicator der Heritage Foundation verwendet. Die ökonomischen und sozialen Rahmenbedingungen eines Landes werden mit einem Punktesystem bewertet. Je restriktiver die Betätigung der Unternehmen und Personen eingeschränkt werden, um so höher fällt die Bepunktung aus. Je höher die erreichte Punktzahl eines Landes, desto weniger ökonomische Freiheiten genießen die Wirtschaftssubjekte. Der Economic Freedom Indicator verwertet Daten zu Korruption, nicht-tarifären Handelshemmnissen, der fiskalischen Situation des Staates (Steuern und Staatsausgaben), Effizienz des Rechtssystems, Regulierungen in den Bereichen Gesundheit, Sicherheit und Umwelt, Restriktionen der Banken, Arbeitsmarktregulierungen sowie informelle Aktivitäten wie Schmuggel oder Schwarzarbeit. ' So ergibt sich ein stark differenziertes Gesamtbild, das auch weiche Faktoren wie die Rechtsprechung erfaßt. Beschränkungen und Regulierungen erzwingen bestimmte, vorgegebene Verhaltensweisen. Damit wird die Suche nach Alternativen verhindert. Könnten die Marktteilnehmer sich unbegrenzt betätigen, so würden sie sich um die Entdeckung von Alternativen bemühen, die ihren Präferenzen entsprechen und kostengünstig zu realisieren sind. Je größer die ökonomischen Freiheiten, die den Akteuren in einem Land gewährt werden, um so mehr Lösungsmöglichkeiten stehen den Marktteilnehmern zur Verfügung. Werden bspw. den Banken sehr strikte Regeln für die Kapitalbeschaffung vorgeschrieben, so erhöht dies tendenziell die Kosten, weil preisgünstigere Alternativen nicht verwendet werden dürfen. Entsprechend hoch sind die resultierenden Zinsmargen. Der Economic Freedom Indicator ist primär ein Messinstrument der Regulierung. Auch vorhandene Protektion, z.B. in Form von Arbeitsmarktregulierungen, die sich gegen ausländische Mitarbeiter richten, wird von ihm erfaßt.

1 Fur weitere Informationen zur Ermittlung dieses jahrlich erstellten Index vgl. http://www. heritage.org, insbes. Beach / Miles 2006. 


\section{Ratings}

Ebenso wie eine hohe Kontrollintensität trägt die ständige Marktbeobachtung durch Rating-Agenturen zu einer Erhöhung der Transparenz und zum Abbau von Informationsasymmetrien bei, wie sie gewöhnlich zwischen Banken einerseits und Unternehmen andererseits existieren. Die positive Bewertung einer Bank bzw. ihrer Produkte kommt einem Qualitätsmerkmal gleich und trägt erheblich zu deren guter Reputation bei. Auf ihren guten Ruf legen Banken sehr viel Wert, da das Vertrauen ihrer Kunden (Unternehmen und Haushalte) in ihre Seriosität von erheblicher Bedeutung für die Geschäftsbeziehungen ist. Die marktüblichen und renommierten Ratings werden von unabhängigen, international operierenden Agenturen erstellt. Sie sind den Anordnungen nationaler Regierungen nicht unterstellt. Deshalb kann eine protektionistisch eingestellte Regierung nicht die von ihr selbst erwünschte Beurteilung der inländischen Unternehmen erzwingen. Folglich beinhaltet diese Variablengruppe kein Protektionspotential. Die Variabeln im Zusammenhang mit Ratings werden aber dennoch getestet, weil sie die Höhe der Zinsmargen durchaus determinieren könnten. Verfügen die Banken eines Landes durchweg über gute Beurteilungen, so ist dies ein Hinweis auf ein stabiles System mit einer eher geringen Krisenanfälligkeit. Entsprechend würden die Zinsen keine Risikoaufschlag enthalten und wären tendenziell niedrig.

\section{Einfluß von staatlichen und ausländischen Vermögensanteilen bei Banken}

Einige Bankenmärkte sind von einem hohen Anteil staatlichen Besitzes am Bankenkapital geprägt. Dieser Umstand erlaubt den Regierungen, erheblichen Einfluß auf den Bankenmarkt zu nehmen. Sie legen nicht nur die gesetzlichen Rahmenbedingungen fest, denen sich alle Banken unterwerfen müssen, sondern steuern auch direkt das Verhalten der von ihr kontrollierten Banken, also eines Teils der Marktteilnehmer. Derart strukturierte Märkte weisen einen eher geringen Wettbewerbsgrad auf. Da jeder zusätzliche Konkurrent die Gewinnmargen der regierungseigenen Banken verringert, werden sie die Zulassung derselbigen tendenziell beschränken.

Eine weitere Variable mißt den Anteil des Bankenvermögens, der sich in ausländischem Besitz befindet. Ist dieser eher hoch, so liegt die Vermutung nahe, daß diese die Zinssätze ihrer Heimatländer importieren. Diese Variablen sind nicht per se in einem protektionistischen Sinne verwendbar, sie könnten aber einen Teil der Zinshöhe bzw. der Zinsmarge erklären, weshalb sie ebenfalls getestet werden.

\section{Allgemeine Befugnisse der Aufsichtsbehörden}

Zur weiteren Verfeinerung der Schätzergebnisse wurden auch die Rechte der Aufsichtsbehörden gegenüber dem Bankenmanagement detaillierter untersucht. Hierzu gehören direkte Zugriffsrechte der Aufsichtsbehörden, z.B. daß sie die Shareholder-Rechte aussetzen oder das Management ablösen und ersetzen können. Protektionistisch verwendet werden können derartige Regulierungen nur, wenn sie diskriminierend gegenüber ausländischen Banken angewendet werden. 


\section{Geschlossene bzw. fusionierte Banken}

Ein hoher Anteil fusionierter Banken kann Anzeichen für einen hohen Wettbewerbsdruck sein, der in einer steigenden Konzentration auf dem Bankenmarkt mündet. Politisch gewollt kann diese Entwicklung im Rahmen einer expansiv orientierten Industriepolitik sein. Nationale Unternehmen werden zu sog. Global Players verschmolzen, um ihre inländische Machtposition verteidigen und ausländische Märkte leichter erobern zu können. Ein hoher Anteil geschlossener Banken kann Hinweis auf eine durchsetzungsstarke Aufsichtsbehörde sein, die ihre Bestimmungen nicht erfüllt sieht und entsprechende Konsequenzen zieht. Für eine exakte Einschätzung der Situation müßten jedoch noch ergänzend weitere relevante Informationen ermittelt und ausgewertet werden. Die Empirie muß klären, ob sich diese Aspekte auf die Zinsmargen auswirken. Insgesamt lassen sich die Ergebnisse dieser Ausführungen tabellarisch wie folgt zusammenfassen:

Tab. 4-1: Übersicht Protektionspotentiale

\begin{tabular}{|l|l|}
\hline Variablengruppen & Protektionspotentiale \\
\hline Kapitalquoten & $\begin{array}{l}\text { bei diskriminierender } \\
\text { Anwendung: Ja }\end{array}$ \\
\hline Attraktivität des Bankenmarktes (Gewinnpotential) & Ja \\
\hline Geschäftsfelder der Banken & Ja \\
\hline Economic Freedom Indicator & Ja \\
\hline Staatliche/ausländische Besitzanteile am Bankenmarkt & Ja \\
\hline Kontrollintensität & unter Umständen \\
\hline Liquidität (im weiteren Sinne) & unter Umständen \\
\hline Einlagensicherungssysteme & unter Umständen \\
\hline Verflechtungsgrad & unter Umständen \\
\hline Anzahl der geschlossenen oder fusionierten Banken & unter Umständen \\
\hline Befugnisse der Aufsichtsbehörde & unter Umständen \\
\hline Eigenkapital der Banken & unter Umständen \\
\hline Konzentrationsgrad & Nein \\
\hline Rechnungslegung / Rechnungsprüfung & Nein \\
\hline Ratings & Nein \\
\hline Offenlegungspflichten / öffentliches Monitoring & Nein \\
\hline $\begin{array}{l}\text { Haftbarkeitjuristische Verantwortung des Banken- } \\
\text { managements }\end{array}$ & Nein \\
\hline & \\
\hline
\end{tabular}


Im folgenden Kapitel werden nun die Ergebnisse der Schätzungen präsentiert und in bezug auf diese Protektionshypothesen diskutiert.

\subsection{Regressionen der Variablengruppen}

\subsubsection{Darstellung der Teilschätzungen}

\subsubsection{Kapitalquoten}

Kapitalquoten dienen als Sicherheitsreserven für unvorhergesehene Entwicklungen, die kurzfristig einen erhöhten Liquiditätsbedarf der Banken bedingen. Die Mindestquoten sind gesetzlich vorgeschrieben. Das Halten darüber hinausgehender Kapitalquoten wird in diesem Zusammenhang zu einem wichtigen Signal. Die Banken reagieren damit auf Risiken, die in ihrer eigenen Aktiva-Passiva-Struktur angelegt sein können, wie auch auf Ungewißheiten, die sich aus einer problematischen ökonomischen Gesamtsituation des Landes ergeben können.

Für einen Vergleich der länderspezifisch unterschiedlich ausgeprägten Bestimmungen hinsichtlich der Kapitalquoten stehen folgende Daten zur Verfügung: ${ }^{1}$

a) Höhe der Mindestkapitalquote (Minimum Capital-Asset-Ratio) (d31),

b) deren Risikogewichtung in Übereinstimmung mit dem Basel-Abkommen von 1988 (d311),

c) Mindestkapitalquote variiert entsprechend des individuellen Kreditrisikos der Bank (d32),

d) Mindestkapitalquote variiert mit dem Marktrisiko (d33),

e) risikobereinigte Kapitalquote gemäß Basel-Abkommen 1988 (d34),

f) Kapitalquote (tatsächlich von den Banken gehalten) (d342),

g) Anteil der Aufwertungs- bzw. Neubewertungsgewinne am Kapital $(\mathrm{d} 36)^{2}$

Ergebnisse für den Zeitraum 1998 - 2000:

Eine deutliche Signifikanz weist nur die Variable d34 ,risikobereinigte Kapitalquote gemäß Basel - Abkommen von 1988“ mit ihrem positiven Vorzeichen auf. Steigt die Kapitalquote, so erhöht sich die Zinsmarge, sinkt die Kapitalquote, so nimmt auch die Zinsmarge ab.

1 Die Umsetzung der Antworten in empirisch verwertbare Größen ist dem Anhang Al zu entnehmen.

2 In den mit ,ja ,oder ,nein“ $\mathrm{zu}$ beantwortenden Fragen wurde die Setzung ,nein"=l und , ja" $=0$ vorgenommen. 
Von nur schwacher Signifikanz und deshalb nicht verläßlich in der Aussage sind die Variablen „Mindestkapitalquotenhöhe“ (d31), „Variation derselben entsprechend des individuellen Banken-Kreditrisikos“ (Variable d32) sowie der „Anteil der Aufwertungsgewinne am Kapital" (Variable d36).

Tab. 4-2: Schätzergebnisse - Kapitalquoten

\begin{tabular}{|c|c|c|c|c|}
\hline & $1998-2000$ & $\begin{array}{l}1998-2000 \text {, } \\
\text { bereinigt }\end{array}$ & $2001-2003$ & $\begin{array}{l}2001-2003 \text {, } \\
\text { bereinigt }\end{array}$ \\
\hline \multirow[t]{2}{*}{ GDPpc } & $* * *-0.0013821$ & $* * *-0.0014081$ & $* * *-0.0007973$ & $* * *-0.0008261$ \\
\hline & $(-5.32)$ & $(-5.74)$ & $(-3.02)$ & $(-3.54)$ \\
\hline \multirow[t]{2}{*}{ d31 } & * -0.4346661 & $*-0.4241115$ & 0.086148 & \\
\hline & $(-1.66)$ & $(-1.73)$ & $(0.46)$ & \\
\hline \multirow[t]{2}{*}{ d311 } & 0.0022015 & & & \\
\hline & $(0.16)$ & & & \\
\hline \multirow[t]{2}{*}{ d32 } & -0.0141603 & $*-0.0123059$ & -0.0009096 & \\
\hline & $(-1.43)$ & $(-1.87)$ & $(-0.11)$ & \\
\hline \multirow[t]{2}{*}{ d33 } & 0.0035052 & & -0.0133741 & $* *-0.0148913$ \\
\hline & $(0.32)$ & & $(-1.40)$ & $(-2.21)$ \\
\hline \multirow[t]{2}{*}{ d34 } & $* * * 0.1765311$ & ***0.177219 & $* * * 0.079365$ & * 0.39122 \\
\hline & (2.78) & $(2.84)$ & $(2.58)$ & (1.77) \\
\hline \multirow[t]{2}{*}{ d342 } & & & 0.037031 & $* * 0.0966755$ \\
\hline & & & $(0.68)$ & (2.16) \\
\hline \multirow[t]{2}{*}{ d36 } & * -0.0128537 & * -0.0125443 & -0.0001707 & \\
\hline & $(-1.81)$ & $(-1.82)$ & $(-0.02)$ & \\
\hline \multirow[t]{2}{*}{ Cons } & * 0.0889936 & $* * * 0.0896919$ & * 0.0326558 & $* * * 0.0413335$ \\
\hline & $(4.32)$ & $(4.68)$ & $(1.64)$ & (4.48) \\
\hline Countries & 58 & 58 & 66 & 85 \\
\hline $\mathrm{R}^{2}$ & 0.4640 & 0.4626 & 0.4115 & 0.3341 \\
\hline Adj. $R^{2}$ & 0.3890 & 0.4109 & 0.3404 & 0.3009 \\
\hline F-Wert & $F(7,50)=6.18$ & $F(5,52)=8.95$ & $F(7,58)=5.79$ & $F(4,80)=10.04$ \\
\hline
\end{tabular}

Ergebnisse für den Zeitraum 2001 - 2003:

Das beste Schätzergebnis liefert die Variable d342, die „tatsächliche Kapitalquote", mit ihrem positiven Vorzeichen und einer Signifikanz zum 5-Prozent-Ni- 
veau. ${ }^{1}$ Ebenfalls signifikant zum 5-Prozent-Niveau ist die Variable „Mindestkapitalquote variiert gemäß Marktrisiken" (d33), die mit einem negativen Koeffizienten in die Schätzung eingeht. $\mathrm{Da}$ in der Schätzung die Ausprägung „nein“ mit 1 gleichgesetzt wurde, bedeutet dies, daß die Mindestkapitalquote nicht individuell an das Kreditrisiko der Bank angepaßt wird. Länder mit fixierten Kapitalquoten weisen in der Regel geringere Zinsmargen auf. Dies könnte ein Hinweis darauf sein, daß Länder mit individuell festgesetzten Kapitalquoten den Banken einen größeren Gestaltungsraum in der Festsetzung der Zinsen einräumen. Dieser könnte in dem Sinne zu eigenen Gunsten, d.h. zur Gewinnerhöhung genutzt werden, so daß die Zinsspreizung vergrößert wird.

Von nur noch schwacher Signifikanz ist die Variable „risikobereinigte Kapitalquote der Banken“ (d34), die im ersten Zeitraum bessere Werte erzielte. Die „tatsächlich gehaltene Kapitalquote der Banken" (Variable d342) determiniert die Zinsmarge eindeutig stärker, sie wurde allerdings im ersten Erhebungszeitraum noch nicht ermittelt.

Tab. 4-3: Signifikanz der Variablen „Kapital“

\begin{tabular}{|c|c|c|c|}
\hline & Variable & $1998-2000$ & $2001-2003$ \\
\hline d31 & Mindestkapitalquote (Høhe) & $(\mathrm{X})$ & \\
\hline d311 & Keine Risikogewichtung nach Basel (1998) & & \\
\hline d32 & $\begin{array}{l}\text { Mindestkapitalquote variiert nicht entsprechend dem } \\
\text { Banken-Kreditrisiko }\end{array}$ & $(\mathrm{X})$ & \\
\hline d33 & Mindestkapitalquote variiert nicht gemaß Marktrisiko & & $\mathbf{X}$ \\
\hline d34 & Risikobereinigte Kapitalquote gemaß Basel (1988) & $\mathbf{X}$ & $(\mathrm{X})$ \\
\hline d342 & Kapitalquote, tatsächlich gehaltene & & $\mathrm{X}$ \\
\hline d36 & Kapitalanteil der Aufwertungsgewinne & $(\mathrm{X})$ & \\
\hline
\end{tabular}

Statistisch läßt sich also belegen, daß die Kapitalquote von starkem Einfluß auf die Höhe der Zinsmargen ist. Allerdings ist nicht die Minimum-Vorschrift von Bedeutung (d31), sondern die höhere, tatsächlich von den Banken gehaltene Quote. Diese spiegelt die Einschätzung der ökonomischen Verhältnisse des Landes durch die Banken wider. Damit begrenzen die Banken aus eigenem Antrieb ihre Kreditvergabe, wodurch die Kreditzinsen bzw. die Zinsmargen steigen.

An Bedeutung verloren hat im Zeitablauf die risikogewichtete Kapitalquote gemäß den Vorgaben des Basel-Abkommens. Die Ursache hierfür kann darin liegen, da $ß$ die entsprechenden Bestimmungen mittlerweile von sehr vielen Ländern umgesetzt wurden.

1 Diese Information wurde in der ersten Datenerhebung nicht ermittelt. 
Für die Wirtschaftspolitik läßt sich die Erkenntnis ableiten, daß die vorgeschriebene Höhe der Mindestkapitalquote eher unerheblich ist. Will sie Einfluß nehmen auf die Gestaltung der Zinsmargen, diese erhöhen oder senken, so muß sie die höhere, tatsächlich von den Banken gehaltene Kapitalquote beeinflussen - bspw. durch die Schaffung stabiler Rahmenbedingungen oder die Reduktion von Unsicherheiten. Es liegt keine Protektion vor, so lange freiwillig hohe Kapitalquoten gehalten werden. Es sei denn, es kommt zu einer diskriminierenden Anwendung geteilter Kapitalquoten, die ausländische Banken benachteiligt. Diese Schlußfolgerung lassen die vorliegenden Daten jedoch nicht zu.

\subsubsection{Attraktivität des Bankenmarktes (Gewinnpotential)}

Ein $\mathrm{Ma} ß$ für die Attraktivität eines Bankenmarktes, seine Gewinn- und Entwicklungsmöglichkeiten stellt die Anzahl in- und ausländischer Bewerbungen um Banklizenzen dar. Hohe Bewerberzahlen signalisieren vorhandene Gewinnpotentiale bzw. Renten, die abgeschöpft werden können. Als Folge vermehrter Markteintritte von Banken steigt der Konkurrenzdruck, er zwingt die Banken zur Entwicklung effizienter administrativer Strukturen, Senkung der Preise und zu weiteren Leistungsverbesserungen. Die Intermediation der Ressource Kapital erfolgt effizienter.

Getestet werden folgende Variablen:

a) Anzahl inländischer Bewerbungen um Banklizenzen (d0171),

b) deren Ablehnung (d0172),

c) Anzahl der ausländischen Bewerbungen (d0181) und

d) deren Ablehnung (d0182).

Ergebnisse für den Zeitraum 1998 - 2000:

Nur die Variable „Ablehnungsquote ausländischer Bewerbungen“ ist schwach signifikant, allerdings nur auf dem 10-Prozent Niveau. Damit sind verläßliche Aussagen kaum möglich. Das negative Vorzeichen des Koeffizienten erscheint darüber hinaus unplausibel, denn das bedeutet, daß bei einer steigenden Ablehnungsquote der Bewerbungen um Banklizenzen die Zinsmarge zunehmend geringer wird. Aber: Je stärker ausländischen Banken der Marktzugang verwehrt wird, um so geringer der Wettbewerbsgrad auf diesem Markt und um so höher müßte die Zinsmarge ausfallen. Wegen ihrer schwachen Signifikanz wird diese Variable verworfen.

Ergebnisse für den Zeitraum 2001 - 2003:

Signifikant, mit negativem Koeffizienten, ist die Variable „Anzahl inländischer Bewerbungen“. Je attraktiver der Bankenmarkt, also je höher die Bewerberzahl 
(und damit kann ein steigender Wettbewerbsgrad unterstellt werden), um so niedriger die Zinsmarge.

Tab. 4-4: Schätzergebnisse - Attraktivität

\begin{tabular}{|c|c|c|c|c|}
\hline & $1998-2000$ & $\begin{array}{l}\text { 1998-2000, } \\
\text { bereinigt }\end{array}$ & $2001-2003$ & $\begin{array}{l}2001-2003, \\
\text { bereinigt }\end{array}$ \\
\hline \multirow[t]{2}{*}{ GDPpc } & $* * *-0.0014542$ & $* * *-0.001274$ & $* *-0.0006479$ & $* *-0.0005969$ \\
\hline & $(-3.94)$ & $(-5.01)$ & $(-2.11)$ & $(-2.07)$ \\
\hline \multirow[t]{2}{*}{$\mathrm{d} 34$} & $* * 0.1468366$ & $* * 0.146597$ & & \\
\hline & $(2.40)$ & (2.76) & & \\
\hline \multirow[t]{2}{*}{ d342 } & & & $* * * 0.2004339$ & $* * 0.1705417$ \\
\hline & & & $(3.60)$ & (3.28) \\
\hline \multirow[t]{2}{*}{ d0171 } & 0.0000305 & & ** -0.0001401 & $* *-0.0001375$ \\
\hline & $(1.38)$ & & $(-2.20)$ & $(-2.26)$ \\
\hline \multirow[t]{2}{*}{ d0172 } & -0.0000806 & & ${ }^{*} 0.0022575$ & $* * 0.0021946$ \\
\hline & $(-0.29)$ & & (1.91) & $(2.06)$ \\
\hline \multirow[t]{2}{*}{ d0181 } & 0.0001172 & & -0.0000575 & \\
\hline & $(0.27)$ & & $(-0.19)$ & \\
\hline \multirow[t]{2}{*}{ d0182 } & -0.0026098 & $*-0.0019756$ & -0.001243 & \\
\hline & $(-1.51)$ & $(-1.66)$ & $(-0.45)$ & \\
\hline \multirow[t]{2}{*}{ Cons } & $* * * 0.0453491$ & $* * * 0.042958$ & $* * * 0.0246861$ & $* * * 0.0242618$ \\
\hline & $(3.87)$ & $(4.47)$ & $(2.57)$ & $(2.90)$ \\
\hline Countries & 42 & 49 & 54 & 60 \\
\hline $\mathrm{R}^{2}$ & 0.5510 & 0.5055 & 0.3904 & 0.3287 \\
\hline Adj. $R^{2}$ & 0.4741 & 0.4726 & 0.3125 & 0.2799 \\
\hline F-Wert & $F(6,35)=7.16$ & $F(3,45)=15.33$ & $F(6,47)=5.02$ & $F(4,55)=6.73$ \\
\hline
\end{tabular}

Signifikant ist ebenfalls die „Ablehnungsquote inländischer Bewerbungen“, allerdings mit positivem Vorzeichen. Auch dieses Ergebnis ist sinnvoll; je niedriger die Ablehnungsquote ausfallt, um so mehr Banken werden zugelassen. Dies erhöht den Wettbewerb auf dem Bankenmarkt und senkt tendenziell die Zinsmargen. 
Tab. 4-5: Signifikanz der Variablen „Attraktivität"

\begin{tabular}{|c|c|c|c|}
\hline Variable & & $1998-2000$ & $2001-2003$ \\
\hline d0171 & $\begin{array}{l}\text { Anzahl inlăndischer Bewerbungen um } \\
\text { Banklizenzen }\end{array}$ & & $\mathbf{X}$ \\
\hline d0172 & deren Ablehnung & & $\mathbf{X}$ \\
\hline d0181 & Anzahl der ausländischen Bewerbungen & & \\
\hline d0182 & deren Ablehnung & $(\mathrm{X})$ & \\
\hline
\end{tabular}

Es bleibt festzuhalten, daß die Anzahl der ausländischen Bewerbungen um Banklizenzen in den betrachteten Zeiträumen keinen Einfluß auf die Zinsmarge nimmt. Im jüngeren Zeitabschnitt ist die statistische Bedeutung der inländischen Bewerbungen für die Zinsmargen erkennbar gestiegen. Eine Erklärung für diesen Umstand kann in den vermehrten Bewerbungen um Banklizenzen liegen. Die Folge ist eine Intensivierung des Wettbewerbs, die die Durchsetzung hoher Zinsmargen verhinderte. $\mathrm{Da} ß$ die erhöhte Nachfrage nach Banklizenzen nur durch in- und nicht auch durch ausländische Banken entstanden ist, beruht wahrscheinlich auf deren besserer Marktkenntnis. Durch die Nähe zum Markt sind inländische Banken eher in der Lage, eine Einschätzung zukünftiger Entwicklungen und der Gewinnpotentiale zu vollziehen. Es ist also davon auszugehen, daß zwischen in- und ausländischen Bewerbern erhebliche Informationsasymmetrien vorliegen. Aber auch die Existenz erheblicher Marktzugangsbarrieren, die sich gegen ausländische Banken richten, kann diese von vornherein von einer Bewerbung um eine Betriebslizenz abhalten.

Eine Erklärung für die stärkere Positionierung der inländischen Banken auf dem heimischen Markt kann im Verlauf der GATS-Verhandlungen gesehen werden. Die absehbaren bzw. bereits beschlossenen Öffnungen der Dienstleistungsmärkte ermutigten die Banken, sich in ihren Ursprungsländern gut zu positionieren, um einerseits ausländische Konkurrenz abwehren zu können und andererseits sich selbst eine solide Basis für die Eroberung ausländischer Marktanteile zu schaffen.

Ergänzend wurde überprüft, ob die Begründungen für die Verweigerung der Lizenzerteilung statistisch signifikant sind. Dies traf bei keinem der folgenden Ablehnungsgründe zu:
a) Mangel an oder Qualität des Kapitals (d0191),
b) Fachkenntnisse / bankspezifische Kenntnisse des Managements (d0192),
c) Reputation (d0193) sowie
d) unvollständige Bewerbungsunterlagen (d0194). 
Im Verlauf von nur wenigen Jahren haben die inländischen Bewerbungen an Bedeutung für die Determinierung der Zinsmarge gewonnen. Dieser Umstand verweist darauf, daß erhebliche Veränderungen der Marktstruktur stattgefunden haben. Die Politik sollte sich um eine hohe Attraktivität des Bankenmarktes bemühen, d.h. eine entsprechende Gestaltung der Rahmenbedingungen vornehmen, um niedrige Zinsmargen zu erreichen und eine hohe Effizienz des Finanzmarktes zu gewährleisten.

Liegt kein verbindlicher Anforderungskatalog vor, der bei Erfüllung automatisch zur Erteilung der Banklizenz führt, so sind willkürliche Einzelfallentscheidungen zu befürchten. Diese Sachlage kann sich für ausländische Banken nachteilig auswirken. Insbesondere wenn ein wirtschaftspolitischer Zielkonflikt zwischen der Wettbewerbs- und der Industriepolitik vorliegt, die die Kreierung möglichst potenter Global Player favorisiert. Einzelfallentscheidungen schaffen den Gestaltungsspielraum, den protektionistische Agenten und Behörden benötigen, um ihre Ziele zu verwirklichen. Die Protektionswirkung einer solchen Regulierung ist durch die obige Schätzung belegt.

\subsubsection{Eigenkapital der Banken}

Ermittelt wird, ob und wie sich Regulierungen hinsichtlich des Eigenkapitals einer Bank auswirken. Hierbei geht es insbesondere um den Aspekt der Konzentration des Eigenkapitals. Die Länder, in denen einige wenige Banken die Finanzintermediation vollständig verantworten, laufen Gefahr, daß marktbeherrschende Konglomerate entstehen. Mangelnder Wettbewerb führt jedoch in der Regel zu ineffizient hohen Preisen für Finanzdienstleistungen.

Aus dem Fragebogen stehen Informationen zur Verfügung bezüglich:

a) Maximaler Eigenkapitalanteil einer einzelnen Person ${ }^{1}$ (d21),

b) Höhe dieses Anteils (d211) und

c) Maximaler Anteil, der von verwandten Personen oder Gesellschaften ${ }^{2}$ gehalten werden darf $(\mathrm{d} 221)$.

Ergebnisse für den Zeitraum 1998 - 2000:

Nur die Beschränkung des Eigenkapitalanteils im Besitz einer Person ist von Signifikanz in der Regression (zum 5-Prozent-Niveau). Der Koeffizient weist ein negatives Vorzeichen auf. Das bedeutet, daß die Existenz einer Höchstbeschränkung sich erhöhend auf die Zinsmarge auswirkt.

1 Die Antwort ,ja“ wurde für die Schătzung mit 1 gleichgesetzt, die Antwort „nein“ mit 0.

2 Darunter werden Verwandte, Geschaftsfreunde, befreundete Unternehmen u.a. verstanden. 
Ergebnisse für den Zeitraum 2001 - 2003:

In diesem Zeitraum ist die einzige signifikante Variable die konkrete Höhe des Eigenkapitalbesitzes durch eine Person. Da ihre Signifikanz allerdings nur schwach ausgeprägt ist, wird auf eine Interpretation verzichtet.

Tab. 4-6: Schätzergebnisse - Eigenkapital

\begin{tabular}{|c|c|c|c|c|}
\hline & $1998-2000$ & $\begin{array}{l}1998-2000 \text {, } \\
\text { bereinigt }\end{array}$ & $2001-2003$ & $\begin{array}{l}2001-2003 \text {, } \\
\text { bereinigt }\end{array}$ \\
\hline \multirow[t]{2}{*}{ GDPpc } & $* * *-0.0018433$ & $* * *-0.0013926$ & $* *-0.000711$ & $* *-0.000717$ \\
\hline & $(-2.95)$ & $(-6.15)$ & $(-2.19)$ & $(-2.45)$ \\
\hline \multirow[t]{2}{*}{ d34 } & 0.1787915 & * 0.0812859 & & \\
\hline & $(1.54)$ & (1.66) & & \\
\hline \multirow[t]{2}{*}{ d342 } & & & 0.0543543 & \\
\hline & & & $(0.74)$ & \\
\hline \multirow[t]{2}{*}{ d21 } & -0.0191506 & ** -0.0115224 & -0.0041519 & \\
\hline & $(-0.93)$ & $(-2.19)$ & $(-0.44)$ & \\
\hline \multirow[t]{2}{*}{ d211 } & 0.0288599 & & $* * 0.0599734$ & * 0.0194336 \\
\hline & $(0.74)$ & & $(2.05)$ & (1.86) \\
\hline \multirow[t]{2}{*}{ d221 } & 0.0135134 & & -0.0385388 & \\
\hline & $(0.46)$ & & $(-1.56)$ & \\
\hline \multirow[t]{2}{*}{ Cons } & $* 0.0457236$ & $* * * 0.0558238$ & $* * * 0.0350429$ & $* * * 0.035588$ \\
\hline & $(1.79)$ & $(6.56)$ & (3.67) & $(6.02)$ \\
\hline Countries & 25 & 63 & 29 & 39 \\
\hline $\mathrm{R}^{2}$ & 0.4600 & 0.4471 & 0.5209 & 0.2035 \\
\hline Adj. $R^{2}$ & 0.3179 & 0.4190 & 0.4168 & 0.1392 \\
\hline F-Wert & $F(5,19)=3.24$ & $F(3,59)=15.91$ & $F(5,23)=5.00$ & $F(2,36)=4.60$ \\
\hline
\end{tabular}

Tab. 4-7: Signifikanz der Variablen „Eigenkapital“

\begin{tabular}{|c|c|c|c|}
\hline Variat & & $1998-2000$ & $2001-2003$ \\
\hline $\mathrm{d} 21$ & Maximaler Eigenkapitalanteil & $\mathbf{X}$ & \\
\hline $\mathrm{d} 211$ & Hohe dieses Anteils & & $(\mathrm{X})$ \\
\hline d221 & $\begin{array}{l}\text { Maximaler Anteil bei verwandten } \\
\text { Gesellschaften/Personen }\end{array}$ & & \\
\hline
\end{tabular}


Der Signifikanzverlust der Variablen d21 („Beschränkung des Eigenkapitals im Besitz einer einzelnen Person") zwischen den beiden Beobachtungszeiträumen läßt sich auf Veränderungen dieser Regelungen in den Ländern zurückführen. Diese Restriktion wurde zunehmend gelockert und verlor an Einfluß auf die Höhe der Zinsmargen.

Insgesamt läßt sich feststellen, daß die hier untersuchten Formen der Eigenkapitalgestaltung mittlerweile keinen signifikanten Einfluß mehr auf die Zinsmargen haben. Somit gehen von den hier behandelten Formen der Gestaltung der Eigentumsverhältnisse in den Banken keine restriktiven oder protektionistischen Einflüsse gegenüber dem Ausland aus. Daten hinsichtlich der Beschränkung des Eigenkapitalbesitzes in ausländischer Hand liegen leider nicht vor. Sie wären sehr interessant, da sich aus ihnen direkte Hinweise für das protektionistische Verhalten eines Landes ergeben würden.

\subsubsection{Verflechtungsgrad zwischen Banken und Finanzunternehmen}

Nun wird untersucht, ob der Grad der Verflechtung zwischen Banken und weiteren, im Finanzsektor tätigen Unternehmen (Versicherungen, Finanzdienstleister etc.) Einfluß auf die Zinsmargen nimmt. In diesem Fall kommen wieder die bereits vorgestellten und miteinander konkurrierenden Argumentationen in Betracht: die Efficient Structure Theory und das Konzept der Marktmacht. Eine starke Konzentration von Finanzunternehmen kann sowohl in einer effizienten Leistungsproduktion wie auch durch die Ausschöpfung der eigenen Marktmacht in monopolartigen Marktlösungen münden. Die Folgen wären hohe Preise, die für Finanzaktiva entrichtet werden müssen, wie auch ein suboptimales Ausmaß an Kapitalangebot und -nachfrage.

Die Frage nach dem Besitz von Geschäftsbanken durch Finanzunternehmen (d25) erlaubte eine Beantwortung in vier Abstufungen:

a) nicht beschränkt: eine Nicht-Bank kann eine Bank zu 100 Prozent besitzen (d252),

b) erlaubt: unbeschränkt nach erfolgter Autorisation / Genehmigung (d251),

c) beschränkt: hinsichtlich des Besitzes von Banken existieren Limitierungen (bspw. Aktienanteile oder Eigenkapitalbeteiligung) (d253) und

d) verboten: Finanzunternehmen ist die Beteiligung am Eigenkapital einer Bank verboten (d254). 
Tab. 4-8: Schätzergebnisse - Verflechtung: Banken und Finanzunternehmen

\begin{tabular}{|c|c|c|c|c|}
\hline & $1998-2000$ & $\begin{array}{l}1998-2000 \text {, } \\
\text { bereinigt }\end{array}$ & $2001-2003$ & $\begin{array}{l}2001-2003 \text {, } \\
\text { bereinigt }\end{array}$ \\
\hline \multirow[t]{2}{*}{ GDPpc } & $* * *-0.0012739$ & $* * *-0.0012724$ & $* * *-0.0006289$ & $* * *-0.0006291$ \\
\hline & $(-5.48)$ & $(-5.56)$ & $(-2.62)$ & $(-2.64)$ \\
\hline \multirow[t]{2}{*}{ d34 } & **0.1010685 & $* * 0.1012588$ & & \\
\hline & $(2.00)$ & $(2.02)$ & & \\
\hline \multirow[t]{2}{*}{ d342 } & & & **** 0.1722987 & ***0.1695951 \\
\hline & & & (3.92) & $(4.00)$ \\
\hline \multirow[t]{2}{*}{ d251 } & 0.0011283 & & 0.0134201 & $* 0.0117571$ \\
\hline & $(0.05)$ & & (1.49) & $(1.91)$ \\
\hline d252 & (dropped) & & (dropped) & \\
\hline \multirow[t]{2}{*}{ d253 } & (dropped) & & 0.0027711 & \\
\hline & & & $(0.25)$ & \\
\hline \multirow[t]{2}{*}{ d254 } & (dropped) & & $* * 0.0261166$ & ** 0.0244454 \\
\hline & & & $(2.09)$ & (2.31) \\
\hline \multirow[t]{2}{*}{ cons } & $* * 0.0459645$ & **** 0.0470335 & 0.014217 & $* * 0.0161531$ \\
\hline & $(2.14)$ & (5.90) & (1.27) & (1.97) \\
\hline Countries & 64 & 64 & 74 & 74 \\
\hline $\mathbf{R}^{2}$ & 0.3907 & 0.3907 & 0.3577 & 0.3571 \\
\hline Adj. $R^{2}$ & 0.3603 & 0.3707 & 0.3105 & 0.3198 \\
\hline F-Wert & $F(3,60)=12.83$ & $F(2,61)=19.56$ & $F(5,68)=7.57$ & $F(4,69)=9.58$ \\
\hline
\end{tabular}

Ergebnisse für den Zeitraum 1998 - 2000:

Keine einzige Variable weist Signifikanz auf. Diese Form der Verflechtung besitzt keine Relevanz für die Höhe der Zinsmargen.

Ergebnisse für den Zeitraum 2001 - 2003:

Stark signifikant für die Determinierung der Zinsmarge ist die Variable d254 "Verbot des Besitzes von Banken durch Finanzunternehmen" mit positivem Vorzeichen und zum 5-Prozent-Niveau. Länder, die ihre Finanzunternehmen mit einem Verbot von derlei Verflechtungen belegen, weisen tendenziell eine höhere Zinsmarge auf als andere. Damit lautet die Schlußfolgerung, daß ein weiteres Voranschreiten der Verflechtung in geringeren Zinsmargen resultiert. Dies wäre ein Hinweis auf die Gültigkeit der Efficient Structure Theory im Bankenmarkt. 
Durch Nutzung von Skaleneffekten in hochkonzentrierten Industrien lassen sich Kosten- und Effizienzvorteile realisieren, die ein atomistisch strukturierter Markt nicht erbringen kann.

Nur schwach signifikant ist der erlaubte Besitz von Banken durch Finanzunternehmen, (zum 10-Prozent-Niveau, ebenfalls mit positivem Koeffizienten), weshalb auf eine Interpretation verzichtet wird.

\section{Tab. 4-9: Signifikanz der Variablen „Verflechtung“}

\begin{tabular}{|c|c|c|c|}
\hline Vari & & $1998-2000$ & $2001-2003$ \\
\hline $\mathrm{d} 251$ & Verflechtung erlaubt & & $(\mathrm{X})$ \\
\hline $\mathrm{d} 252$ & ............nicht beschränkt & & \\
\hline $\mathrm{d} 253$ & ............beschrănkt & & \\
\hline d254 & ............verboten & & $\mathrm{X}$ \\
\hline
\end{tabular}

Ergänzend sei angemerkt, daß die Untersuchung des Verflechtungsgrades zwischen Banken und Nicht-Finanzunternehmen (analog zu der eben dargestellten Regression) keinerlei Signifikanz erbrachte. Der Besitz von Banken durch Industrieunternehmen hat somit keinen statistisch meßbaren Einfluß auf die Zinsmargen. Synergien lassen sich also nur nutzen, wenn die kooperierenden Unternehmen im Finanzsektor tätig sind.

Für die Wirtschaftspolitik läßt sich schlußfolgern, daß durch die starke Verflechtung von Banken und anderen Finanzunternehmen nicht so sehr wettbewerbsbehindernde Folgen zu fürchten sind, als daß vielmehr die Effizienz des Finanzmarktes erhöht wird. Sollte ein hoher Effizienzgrad Ziel der Wirtschaftspolitik sein, so sind Verflechtungen dieser Art zu fördern.

Aber auch in diesem Fall ist bei Fehlen einer konsistenten wirtschaftspolitischen Richtlinie ein Zielkonflikt unausweichlich: Während die wettbewerbsorientierten Akteure für die Machtstreuung und Entflechtung eintreten werden, um einer schleichenden Monopolisierung in der Zukunft vorzubeugen, werden die Vertreter einer expansiven Industriepolitik, den Verflechtungsgrad zu steigern suchen. Damit sollen die inländischen Unternehmen möglichst gut für die Eroberung ausländischer Marktanteile oder den Erwerb von ausländischen Konkurrenzunternehmen gerüstet werden. Die Förderung dieser Verflechtungen ist auf verschiedenen Ebenen möglich, bspw. durch günstige staatliche Kredite oder Steuervorteile. Das Protektionspotential dieser Regelung ist entsprechend groß. 


\subsubsection{Konzentrationsgrad}

Eng verbunden mit der Frage nach dem Verflechtungsgrad ist der Aspekt des Konzentrationsgrades eines Marktes. Auch hier stehen die zwei konkurrierenden Lehrmeinungen Efficient Structure Theory und das Konzept der Marktmacht gegenüber. Der Konzentrationsgrad wird von drei Indikatoren erfaßt:

a) Anteil der 5 größten Banken an den Depositen der Geschäftsbanken (d27),

b) Anteil der 5 größten Geschäftsbanken am Gesamtvermögen (Assets) (d28) sowie

c) Vermögensanteil der Geschäftsbanken bezogen auf alle Depositeninstitute (d29).

Ergebnisse für den Zeitraum 1998 - 2000:

In der ersten Erhebung wurde nur ein einziges Konzentrationsmaß ermittelt, der „Anteil der 5 größten Banken an den Depositen der Geschäftsbanken“ (Variable d27). Für die Schätzung ist diese Variable insignifikant: Dieses Konzentrations$\mathrm{maß}$ beeinflußt die Zinsmargen in dieser Periode nicht.

Ergebnisse für den Zeitraum 2001 - 2003:

Sowohl Variable d27 (Konzentration der Depositen) wie auch d28 (Konzentration der Assets) sind zum 5-Prozent-Niveau signifikant. Allerdings weist der Konzentrationsgrad gemessen anhand der Depositen einen negativen Koeffizienten auf. Je höher die Konzentration der Einlagen, desto geringer ist die Zinsmarge. Eine mögliche Interpretation ist: Die hohe Konzentration der Einlagen erleichtert die effiziente Bereitstellung von hohen Kreditvolumina. Damit entfällt eine kostenintensive Kapitalbeschaffung, die u.U. auf dem internationalen Kapitalmarkt getätigt werden müßte. Geringe Kosten drücken sich in niedrigen Zinsmargen aus.

Im Gegensatz hierzu steht das asset-basierte Konzentrationsmaß in einem positiven Zusammenhang mit der Zinsmarge. Eine zunehmende Vermögenskonzentration läßt die Zinsmarge steigen, was wiederum Rückschlüsse auf eine marktbeherrschende Stellung der Kreditinstitute zuläßt. Die Banken sind in der Lage, die Zinsspreizung zu eigenen Gunsten zu vergrößern. Somit lassen sich für die beiden konkurrierenden Erklärungsansätze in diesem Datensatz durchaus Belege finden. Welche Variable die Zusammenhänge besser abbildet, kann nur in einer erweiterten Betrachtung entschieden werden, in der Ermittlung der aussagekräftigsten Gesamtschätzung. ${ }^{1}$ Die Tatsache, daß die Stärke der Konzentration in der ersten Periode nicht signifikant war, ist überraschend. Die Autorin ist zunächst von einer hohen Bedeutung dieser Kennzahl für die Determinierung der Zinsmargen ausge-

1 Vgl. hierzu Kapitel 4.2.2 Aussagekrafftigste Gesamtschătzungen. 
gangen. Erst im zweiten Untersuchungszeitraum gewannen die neu hinzugetretenen Konzentrationsmaße an Bedeutung. Es ist zu vermuten, daß Veränderungen in den Bankenmarktbedingungen stattfanden, so daß die Auswirkungen der Konzentrationsmaße erst im späteren Zeitraum zur Geltung kamen.

Tab. 4-10: Schätzergebnisse - Konzentrationsgrad

\begin{tabular}{|c|c|c|c|c|}
\hline & $1998-2000$ & $\begin{array}{l}1998-2000, \\
\text { bereinigt }\end{array}$ & $2001-2003$ & $\begin{array}{l}2001-2003 \text {, } \\
\text { bereinigt }\end{array}$ \\
\hline \multirow[t]{2}{*}{ GDPpc } & $* * *-0.0012039$ & $* * *-0.0012724$ & $* *-0.0006196$ & $* * *-0.000635$ \\
\hline & $(-4.51)$ & $(-5.56)$ & $(-2.36)$ & $(-2.87)$ \\
\hline \multirow[t]{2}{*}{$\mathrm{d} 34$} & $* * 0.1252737$ & ${ }^{* *} 0.1012588$ & & \\
\hline & $(2.31)$ & $(2.02)$ & & \\
\hline \multirow[t]{2}{*}{ d342 } & & & $* * * 0.1823959$ & $* * * 0.1936854$ \\
\hline & & & $(2.67)$ & (4.31) \\
\hline \multirow[t]{2}{*}{$d 27$} & 0.010955 & & $* *-0.1261744$ & ** -0.121344 \\
\hline & $(0.82)$ & & $(-2.18)$ & $(-2.31)$ \\
\hline \multirow[t]{2}{*}{$\mathrm{d} 28$} & & & $* * 0.1215433$ & $* * 0.1178142$ \\
\hline & & & $(2.17)$ & (2.31) \\
\hline \multirow[t]{2}{*}{ d29 } & & & 0.002723 & \\
\hline & & & $(0.21)$ & \\
\hline \multirow[t]{2}{*}{ cons } & *** 0.0362979 & $* * * 0.0470335$ & 0.0284145 & ** 0.0286917 \\
\hline & $(2.92)$ & (5.90) & $(1.60)$ & (2.50) \\
\hline Countries & 57 & 64 & 69 & 82 \\
\hline $\mathrm{R}^{2}$ & 0.4065 & 0.3907 & 0.2759 & 0.3458 \\
\hline Adj. $R^{2}$ & 0.3730 & 0.3707 & 0.2185 & 0.3118 \\
\hline F-Wert & $F(3,53)=12.10$ & $F(2,61)=19.56$ & $F(5,63)=4.80$ & $F(4,77)=10.18$ \\
\hline
\end{tabular}

Tab. 4-11: Signifikanz der Variablen „Konzentrationsgrad“

\begin{tabular}{|c|c|c|c|}
\hline Vari & & $1998-2000$ & $2001-2003$ \\
\hline d27 & Anteil der 5 grőßten Banken an den Depositen & & $\mathrm{X}$ \\
\hline $\mathrm{d} 28$ & $\begin{array}{l}\text { Anteil der } 5 \text { großten Geschăftsbanken am } \\
\text { Gesamtvermögen }\end{array}$ & & $\mathbf{x}$ \\
\hline d29 & Vermögensanteil der Geschăftsbanken & & \\
\hline
\end{tabular}


Diese Ergebnisse ermöglichen nicht die Formulierung eindeutiger Empfehlungen für die Wirtschaftspolitik. Bei Priorität für eine niedrige Zinsmarge sollte die Depositenkonzentration gefördert werden, bspw. über die zahlenmäßige Beschränkung von Sparkassen oder hierin spezialisierter Banken. Eine andere Möglichkeit, dieses Ziel zu verwirklichen, stellt die Beschränkung der Akkumulation von Banken-Assets in der Hand weniger Banken dar. In diesem Fall muß eine möglichst breitgefächerte, dezentrale Verteilung des Bankenvermögens realisiert werden.

\subsubsection{Geschäftsfelder der Banken}

Ob sich die Beschränkung von Bankaktivitäten auf die Zinsmargen auswirken, wird anhand der Regulierungen hinsichtlich des Wertpapier-, Versicherungs- und Immobiliengeschäfts gemessen. Grundsätzlich ist zu vermuten, daß die Erlaubnis zur Betätigung in diesen, dem Bankengeschäft angrenzenden Bereichen von reduzierender Wirkung auf die Zinsmargen ist. Diese Geschäftsfelder stellen Ergänzungen der Hauptbetätigung, der Kreditvermittlung, und damit neue Gewinnquellen dar. Die Banken können Verbundvorteile nutzen und ihr unternehmerisches Gesamtrisiko diversifizieren. Entsprechend sollten Länder, die ihren Banken vielfältige Betätigungsmöglichkeiten genehmigen, tendenziell niedrigere Zinsmargen aufweisen.

Die möglichen Ausprägungen der Variablen lauten:

- Unrestricted (nicht beschränkt): Alle nötigen Handlungen können direkt in der Bank erfolgen.

- Permitted (erlaubt): Damit zusammenhängende Aktivitäten dürfen ausgeführt werden, aber sie müssen (zumindest zum Teil) in Tochterbanken erfolgen.

- Restricted (beschränkt): Nicht alle Vorgänge können in der Bank oder einer Tochtergesellschaft erfolgen.

- Prohibited (verboten): Die Vorgänge dürfen weder in der Bank noch bei einer Tochter erfolgen.

a) Den Banken sind Wertpapiergeschäfte ${ }^{1}$ :

Nicht beschränkt (d412), erlaubt (d411), beschränkt (d413), verboten $(\mathrm{d} 414)$.

1 Darunter werden Tătigkeiten erfaßt wie die Emission, die Vermittlung und der Handel von Effekten, Investmentfonds etc. 
b) Versicherungsgeschäfte: ${ }^{1}$

$\begin{array}{ll}\text { Nicht beschränkt (d422), } & \text { erlaubt }(\mathrm{d} 421), \\ \text { beschränkt }(\mathrm{d} 423), & \text { verboten }(\mathrm{d} 424) .\end{array}$

c) Immobiliengeschäfte: ${ }^{2}$

Nicht beschränkt (d432), erlaubt (d431),

beschränkt (d433), verboten ( 1434$)$.

d) Banken dürfen Unternehmen (nicht aus dem Finanzbereich) besitzen:

Nicht beschränkt: Bank darf 100 Prozent des Eigenkapitals besitzen (d442),

Erlaubt: $\quad$ Bank kann 100 Prozent des Firmeneigenkapitals besitzen, aber der Anteil ist begrenzt auf die Höhe des Bankeneigenkaptals (d441),

Beschränkt: $\quad$ Bank darf nur weniger als 100 Prozent des Eigenkapitals besitzen (d443),

Verboten: $\quad$ Bank darf gar keine anderen Unternehmen besitzen (d444).

Ergebnisse für den Zeitraum 1998 - 2000:

Von hoher Signifikanz sind die Variablen d411 ,erlaubter Wertpapierhandel“" (negativer Koeffizient, 5-Prozent-Niveau) sowie d412 „nicht beschränkter Wertpapierhandel" (negatives Vorzeichen, 1-Prozent-Niveau). Der negative Zusammenhang zwischen der Betätigung der Banken im Wertpapierhandel und den Nettozinsmargen verdeutlicht, daß mit steigendem Ausbau dieser Aktivität die Zinsmargen sinken. Diejenigen Länder, die den Banken den Wertpapierhandel ermöglichen, zeichnen sich durch eine vergleichsweise niedrige Zinsmarge aus.

Die Variable d441 „Unternehmen in Bankenbesitz: erlaubt“ hingegen weist ein positives Vorzeichen auf (5-Prozent-Niveau). Je mehr Banken mit den inländischen Unternehmen verstrickt sind, um so höher sind die Zinsmargen. Dies kann ein Hinweis auf eine starke Verflechtung sein, die den Banken eine hohe Marktmacht einräumt. Dadurch sind Banken in der Lage, die Zinsmargen zu ihren Gunsten zu gestalten, d.h. sie zu erhöhen.

1 Darunter fallen die Abschlusse von Versicherungsvertrăgen und deren Handel.

2 Dieser Bereich umfaßt die Bereiche Investition, Entwicklung sowie Immobilien-Management. 
Tab. 4-12: Schätzergebnisse - Geschäftsfelder der Banken

\begin{tabular}{|c|c|c|c|c|}
\hline & $1998-2000$ & $\begin{array}{l}1998-2000 \text {, } \\
\text { bereinigt }\end{array}$ & $2001-2003$ & $\begin{array}{l}2001-2003, \\
\text { bereinigt }\end{array}$ \\
\hline \multirow[t]{2}{*}{ GDPpc } & $* * *-0.0010869$ & $* * *-0.0011239$ & $* * *-0.000671$ & $* * *-0.0006536$ \\
\hline & $(-3.91)$ & $(-5.39)$ & $(-2.57)$ & $(-2.74)$ \\
\hline \multirow[t]{2}{*}{ d34 } & ${ }^{* *} 0.1292498$ & **** 0.1205329 & & \\
\hline & (2.43) & (2.69) & & \\
\hline \multirow[t]{2}{*}{ d342 } & & & $* * 0.1299682$ & $* * * 0.1385448$ \\
\hline & & & $(2.61)$ & (3.23) \\
\hline \multirow[t]{2}{*}{ d411 } & ${ }^{* *}-0.0245807$ & ** -0.0182634 & 0.0011999 & \\
\hline & $(-2.10)$ & $(-2.52)$ & $(0.13)$ & \\
\hline \multirow[t]{2}{*}{ d412 } & $* * *-0.0140677$ & $* * *-0.0349179$ & -0.0093631 & $*-0.0095081$ \\
\hline & $(-3.72)$ & $(-4.92)$ & $(-1.04)$ & $(-1.74)$ \\
\hline d413 & (dropped) & & (dropped) & \\
\hline \multirow[t]{2}{*}{ d414 } & 0.0206187 & & -0.0027944 & \\
\hline & $(0.94)$ & & $(-0.10)$ & \\
\hline \multirow[t]{2}{*}{ d421 } & -0.0002586 & & -0.001965 & \\
\hline & $(-0.02)$ & & $(-0.19)$ & \\
\hline d422 & (dropped) & & (dropped) & \\
\hline \multirow[t]{2}{*}{ d423 } & -0.0035072 & & -0.0022218 & \\
\hline & $(-0.25)$ & & $(-0.21)$ & \\
\hline \multirow[t]{2}{*}{ d424 } & -0.0008238 & & 0.0010732 & \\
\hline & $(-0.06)$ & & $(0.09)$ & \\
\hline \multirow[t]{2}{*}{ d431 } & -0.0006762 & & & \\
\hline & $(-0.07)$ & & & \\
\hline \multirow[t]{2}{*}{ d432 } & -0.0010205 & & & \\
\hline & $(-0.08)$ & & & \\
\hline \multirow[t]{2}{*}{ d433 } & -0.0022183 & & & \\
\hline & $(-0.21)$ & & & \\
\hline \multirow[t]{2}{*}{ d434 } & -0.0012023 & & -0.0030807 & \\
\hline & $(-0.09)$ & & $(-0.49)$ & \\
\hline
\end{tabular}


Tab. 4-12: Schätzergebnisse - Geschäftsfelder der Banken (Forts.)

\begin{tabular}{|c|c|c|c|c|}
\hline & $1998-2000$ & $\begin{array}{l}1998-2000, \\
\text { bereinigt }\end{array}$ & $2001-2003$ & $\begin{array}{l}2001-2003 \text {, } \\
\text { bereinigt }\end{array}$ \\
\hline \multirow[t]{2}{*}{ d441 } & 0.0064343 & $* * 0.0103735$ & & \\
\hline & $(0.85)$ & (2.24) & & \\
\hline d442 & (dropped) & & & \\
\hline \multirow[t]{2}{*}{ d443 } & -0.0053918 & & & \\
\hline & $(-0.67)$ & & & \\
\hline \multirow[t]{2}{*}{ d444 } & 0.0029182 & & -0.0007312 & \\
\hline & $(0.21)$ & & $(-0.11)$ & \\
\hline \multirow[t]{2}{*}{ cons } & $* * * 0.0735597$ & $* * * 0.063931$ & $* * 0.0371764$ & $* * * 0.0337395$ \\
\hline & (3.41) & $(6.81)$ & $(2.54)$ & (5.30) \\
\hline Countries & 64 & 64 & 86 & 87 \\
\hline $\mathrm{R}^{2}$ & 0.6119 & 0.5966 & 0.2887 & 0.2836 \\
\hline Adj. $R^{2}$ & 0.4906 & 0.5618 & 0.1939 & 0.2577 \\
\hline F-Wert & $F(15,48)=5.04$ & $F(5,58)=17.16$ & $F(10,75)=3.04$ & $F(3,83)=10.95$ \\
\hline
\end{tabular}

Ergebnisse für den Zeitraum 2001 - 2003:

In diesem Zeitraum ist nur noch die Variable $\mathrm{d} 412$,nicht beschränkter Wertpapierhandel" schwach signifikant und zwar wiederum mit einem negativen Vorzeichen (10-Prozent-Niveau); ein Ergebnis das nur sehr vorsichtig interpretiert werden darf.

Insgesamt erlitt die Variable „Wertpapierhandel“ einen Bedeutungsverlust in der Determinierung der Höhe der Nettozinsmargen. Eine mögliche Erklärung hierfür ist, daß mit voranschreitender Internationalisierung des Bankengeschäfts Beschränkungen dieser Aktivität zunehmend abgebaut wurden.

Die Beschränkungen der geschäftlichen Aktivitäten der Banken lassen sich mittlerweile kaum noch an einer höheren Zinsmarge ablesen. Sie scheinen keinen (zumindest keinen meßbaren) zinsdivergierenden Einfluß auszuüben. Die Bereiche Versicherungs- und Immobiliengeschäfte hatten im gesamten Beobachtungszeitraum keinen Einfluß auf die Zinsmarge. Auch die Beteiligungen der Banken an (Nicht-Finanz-)Unternehmen beeinflussen die Zinssetzung nicht mehr. Eine mögliche Erklärung hierfür ist, daß die Länder mittlerweile zu einem überwiegenden Teil derlei Beschränkungen abgeschafft haben. 
Tab. 4-13: Signifikanz der Variablen „Geschäftsfelder der Banken“

\begin{tabular}{l|l|c|c}
\multicolumn{1}{l|}{ Variable } & $1998-2000$ & $2001-2003$ \\
\hline $\mathrm{d} 411$ & erlaubt & $X$ & \\
$\mathrm{~d} 412$ & nicht beschrănkt & $X$ & $(X)$ \\
$\mathrm{d} 413$ & beschrănkt & & \\
$\mathrm{d} 414$ & verboten & & \\
$\mathrm{d} 421$ & Versicherungen & erlaubt & \\
$\mathrm{d} 422$ & nicht beschrănkt & & \\
$\mathrm{d} 423$ & beschrănkt & & \\
$\mathrm{d} 424$ & verboten & & \\
$\mathrm{d} 431$ & Immobilien & erlaubt & \\
$\mathrm{d} 432$ & nicht beschrănkt & & \\
$\mathrm{d} 433$ & beschrănkt & & \\
$\mathrm{d} 434$ & verboten & X Signifikanz, (X) = schwache Signifikanz.
\end{tabular}

Für die Wirtschaftspolitik folgt daraus: Je freizügiger den Banken die Betätigung in Geschäftsfeldern wie dem Wertpapierhandel erlaubt wird, um so geringer die resultierende Zinsmarge des Landes. Die Kombination von Bank- und Versicherungsleistungen, die zeitweise großen Widerhall in der betriebswirtschaftlichen Diskussion unter dem Stichwort der All-Finanz-Unternehmen (Nutzung von Synergien) fand, hat - zumindest mit Blick auf die Zinshöhe - an Relevanz verloren.

Unter dem Aspekt der Protektion ist auf die GATS-Regulierungen zu verweisen. Während in Deutschland der Typ der Universalbank vorherrscht und Banken in der Regel alle Dienstleistungen parallel anbieten dürfen und zumeist auch erbrin- 
gen, weisen andere Länder oftmals eine stark spezialisierte Bankenlandschaft auf. ${ }^{1}$ So bedienen Hypothekenbanken den Kreditmarkt für Immobilien, nicht aber bspw. das Emissionsgeschäft. Aufgrund der eigenen tradierten Strukturen nutzen diese Länder die Möglichkeit, ausländischen Banken die gleichzeitige Betätigung in mehreren Geschäftsbereichen zu verbieten. Das Protektionspotential dieser Regelung wird also praktisch ausgeschöpft.

\subsubsection{Rechnungslegung}

Die Bilanzen der Banken stellen eine wichtige Informationsquelle für verschiedene Interessensgruppen dar. Vorschriften, die eine korrekte Bilanzierung der Banken sicherstellen, wirken sich im sensiblen Finanzsektor positiv auf das Vertrauen der Marktteilnehmer aus. Ob sich in den Vorschriften hinsichtlich der Rechnungslegung wettbewerbsbeschränkende Bestimmungen auswirken, wird gemessen anhand der Variablen: ${ }^{2}$

a) Verpflichtende Bilanzprüfung (d51),

b) Spezielle Anforderungen an die Bilanzprüfung (d52),

c) Lizenzierte Bilanzprüfer (d53),

d) Meldung der Ergebnisse an die Aufsichtsbehörden (d54),

e) Aufsichtsbehörden können die Bilanzprüfer zwecks Diskussion des Prüfberichts ohne Genehmigung der Betroffenen kontaktieren (d55),

f) Pflichtmeldung der Bilanzprüfer bei der Aufsichtsbehörde bei Verdacht auf eine Straftat oder Hinterziehung (d56),

g) Aufsichtsbehörde kann bei Fahrlässigkeit juristisch gegen Bilanzprüfer vorgehen (d57),

h) Ist dieses in den letzten 5 Jahren vorgekommen? (d58).

Ergebnisse für den Zeitraum 1998 - 2000:

Keine einzige Variable weist signifikante Schätzer auf.

Ergebnisse für den Zeitraum 2001 - 2003:

Eindeutig signifikant zum 5-Prozent-Niveau ist die Variable d56 „keine Meldepflicht der Bilanzprüfer bei Verdacht auf Betrug“. Sie geht mit einem positiven

1 In den USA wandelt sich diese strikte Differenzierung der einzelnen Banken, aber grundsătzlich sind die Trennungen der Geschaftsbereiche in auf Aktiengeschäfte spezialisierte und Hypothekenbanken noch gut nachvollziehbar.

2 Bei allen Variablen wurden die Antworten fur die Regression umgesetzt in "nein" $=1$, ,ja“ = 0. 
Tab. 4-14: Schätzergebnisse - Rechnungslegung

\begin{tabular}{|c|c|c|c|c|}
\hline & $1998-2000$ & $\begin{array}{l}1998-2000 \text {, } \\
\text { bereinigt }\end{array}$ & $2001-2003$ & $\begin{array}{l}2001-2003 \text {, } \\
\text { bereinigt }\end{array}$ \\
\hline \multirow[t]{2}{*}{ GDPpc } & $* * *-0.0008815$ & $* * * * 0.0012724$ & $* * *-0.000791$ & $* * *-0.0007451$ \\
\hline & $(-3.04)$ & $(-5.56)$ & $(-3.41)$ & $(-3.30)$ \\
\hline \multirow[t]{2}{*}{ d34 } & 0.0539472 & $* * 0.1012588$ & & \\
\hline & $(0.94)$ & (2.02) & & \\
\hline \multirow[t]{2}{*}{ d342 } & & & **** 0.1168032 & *** 0.1341217 \\
\hline & & & $(2.72)$ & (3.25) \\
\hline \multirow[t]{2}{*}{ d51 } & (dropped) & & -0.0055832 & \\
\hline & & & $(-0.23)$ & \\
\hline \multirow[t]{2}{*}{ d52 } & -0.0160041 & & ${ }^{*}-0.0162487$ & \\
\hline & $(-1.47)$ & & $(-1.84)$ & \\
\hline \multirow[t]{2}{*}{ d53 } & (dropped) & & 0.00087317 & \\
\hline & & & $(0.49)$ & \\
\hline \multirow[t]{2}{*}{ d54 } & 0.0080786 & & (dropped) & \\
\hline & $(0.31)$ & & & \\
\hline \multirow[t]{2}{*}{ d55 } & 0.0001987 & & 0.0124838 & \\
\hline & $(0.02)$ & & (1.57) & \\
\hline \multirow[t]{2}{*}{ d56 } & 0.0069781 & & 0.0161985 & **0.0145415 \\
\hline & $(0.71)$ & & (2.32) & (2.46) \\
\hline \multirow[t]{2}{*}{ d57 } & -0.0070905 & & -0.0069748 & \\
\hline & $(-0.32)$ & & $(-1.07)$ & \\
\hline \multirow[t]{2}{*}{ d58 } & 0.0052876 & & $* * *-0.0180359$ & *** -0.0205203 \\
\hline & $(0.65)$ & & $(-2.61)$ & $(-3.18)$ \\
\hline \multirow[t]{2}{*}{ cons } & **** 0.0427653 & $* * * 0.0470335$ & $* * * 0.0462969$ & $* * 0.043635$ \\
\hline & $(3.91)$ & $(5.90)$ & (5.80) & (5.69) \\
\hline Countries & 35 & 64 & 82 & 83 \\
\hline $\mathrm{R}^{2}$ & 0.3837 & 0.3907 & 0.4229 & 0.3783 \\
\hline Adj. $R^{2}$ & 0.1941 & 0.3707 & 0.3508 & 0.3464 \\
\hline F-Wert & $F(8,26)=2.02$ & $F(2,61)=19.56$ & $F(9,72)=5.86$ & $F(4,78)=11.87$ \\
\hline
\end{tabular}

Vorzeichen in die Regression ein. Eine hohe Zinsmarge ist also auch ein Indikator für qualitativ mangelhafte Kontrollen seitens der Bilanzprüfer. Länder, die diese 
Pflichtmeldung in ihren Bestimmungen vorsehen, zeichnen sich durch eine niedrigere Zinsmarge aus. Die Kontrollen sind effektiver und drohende Anzeigen verhindern, daß die Banken ihren Bilanzierungsspielraum mißbrauchen.

Für die Variable d58, ,gerichtliches Vorgehen der Aufsichtsbehörden gegen fahrlässige Bilanzprüfer" ermittelt die Regression einen negativen Koeffizienten (1Prozent-Niveau). Länder, die nicht gegen ihre fahrlässig agierenden Bilanzprüfer vorgehen, haben eine niedrige Zinsmarge. Die Argumentation muss eher umgekehrt erfolgen: Die Länder, die niedrige Zinsmargen aufweisen, verfügen in der Regel über ein gut ausgebautes Kontrollsystem und gut ausgebildete Bilanzprüfer. Das Verhältnis zwischen Wirtschaftsprüfungsgesellschaften und Klienten ist von einem hohen Vertrauen geprägt; die Prüfer sind sehr auf einen guten Ruf bedacht und verhalten sich in der Regel nicht fahrlässig. Deshalb besteht auch sehr viel weniger Notwendigkeit, gegen diese juristisch vorgehen zu müssen.

\section{Tab. 4-15: Signifikanz der Variablen „Rechnungslegung“}

\begin{tabular}{|c|c|c|c|}
\hline Vari & & $1998-2000$ & $2001-2003$ \\
\hline $\mathrm{d} 51$ & Keine verpflichtende Bilanzprufung & & \\
\hline d52 & Keine speziellen Anforderungen fur die Bilanzprufung & & \\
\hline d53 & Lizenzierte Bilanzprufer & & \\
\hline d54 & Meldung der Ergebnisse an die Aufsichtsbehörden & & \\
\hline d55 & Kontakt zw. Aufsichtsbehørden \& Bilanzprufern & & \\
\hline d56 & Keine Meldepflicht fur die Bilanzprufer & & $\mathbf{X}$ \\
\hline d57 & Bei Fahrlässigkeit: juristische Folgen & & \\
\hline d58 & Ist dieses in den letzten 5 Jahren vorgekommen & & $\mathbf{X}$ \\
\hline
\end{tabular}

Die Bewehrung des Fehlverhaltens der Bilanzprüfer mit Strafen führt zu effektiven Kontrollen; diese schlagen sich in niedrigen Zinsmargen nieder. Die Ausgestaltung des Kontrollsystems hat für die spätere Periode erheblich an Bedeutung gewonnen.

Wirtschaftspolitiker sollten sich folglich um die Einrichtung eines wirksamen Kontrollsystems bemühen und die strikte Überwachung der Banken veranlassen, wenn sie die Zinsmargen ihres Landes gering halten wollen.

\subsubsection{Liquidität (im weiteren Sinne)}

Die Haltung liquider Mittel dient den Banken zur Überbrückung kurzfristiger finanzieller Engpässe. Diese Reserven können nicht zinstragend investiert werden 
Tab. 4-16: Schätzergebnisse - Liquidität

\begin{tabular}{|c|c|c|c|c|}
\hline & $1998-2000$ & $\begin{array}{l}1998-2000 \text {, } \\
\text { bereinigt }\end{array}$ & $2001-2003$ & $\begin{array}{l}2001-2003 \text {, } \\
\text { bereinigt }\end{array}$ \\
\hline \multirow[t]{2}{*}{ GDPpc } & $* * *-0.0012138$ & $* * *-0.0010456$ & $* *-0.000754$ & $* * *-0.000751$ \\
\hline & $(-3.67$ & $(-4.53)$ & $(-2.14)$ & $(-3.20)$ \\
\hline \multirow[t]{2}{*}{ d34 } & 0.0936894 & **** 0.1380916 & & \\
\hline & $(1.02$ & (2.81) & & \\
\hline \multirow[t]{2}{*}{ d342 } & & & ${ }^{* * *} 0.1532003$ & *** 0.1303979 \\
\hline & & & (2.96) & $(3.02)$ \\
\hline \multirow[t]{2}{*}{ d71 } & 0.0011257 & & 0.0058902 & \\
\hline & $(0.15$ & & $(0.92)$ & \\
\hline \multirow[t]{2}{*}{ d72 } & 0.0149869 & ${ }^{* * *} 0.0198848$ & 0.0048673 & \\
\hline & $(1.56$ & $(2.85)$ & $(0.48)$ & \\
\hline \multirow[t]{2}{*}{ d731 } & -0.009677 & & -0.0305414 & \\
\hline & $(-0.68$ & & $(-0.49)$ & \\
\hline \multirow[t]{2}{*}{ d74 } & -0.0044249 & & -0.0073803 & \\
\hline & $(-0.59$ & & $(-1.10)$ & \\
\hline \multirow[t]{2}{*}{ d753 } & & & 0.0002541 & \\
\hline & & & $(0.04)$ & \\
\hline \multirow[t]{2}{*}{ d754 } & & & -0.0172494 & \\
\hline & & & $(-1.18)$ & \\
\hline \multirow[t]{2}{*}{ cons } & $* * * 0.0462917$ & $* * * 0.0361767$ & $* * * 0.0361681$ & $* * 0.0317009$ \\
\hline & (2.91) & $(4.28)$ & (2.76) & $(5.00)$ \\
\hline Countries & 41 & 64 & 65 & 87 \\
\hline $\mathrm{R}^{2}$ & 0.4198 & 0.4633 & 0.2826 & 0.2573 \\
\hline Adj. $R^{2}$ & 0.3174 & 0.4364 & 0.1802 & 0.2397 \\
\hline F-Wert & $F(6,34)=4.10$ & $F(3,60)=17.26$ & $F(8,56)=2.76$ & $F(2,84)=14.55$ \\
\hline
\end{tabular}

und senken somit das Gewinnpotential einer Bank. Die Beschränkungen können hinsichtlich der Art und Zusammensetzung der liquiden Mittel wie auch ihrer Beschaffung im Ausland vorliegen. Geprüft werden die Aspekte: 
a) Verpflichtende Diversifizierung der Assets (d71),

b) Verbot für Banken, Anleihen im Ausland zu begeben (d72),

c) Halten einer Mindestliquidität ist vorgeschrieben (d731),

d) Höhe der vorgeschriebenen Mindestliquidität (d74),

e) Dürfen Banken Reserven in Form ausländischer Währungen oder anderen ausländischen Instrumenten halten? (d753),

f) Anteil der in ausländischen Währungen denominierten Assets der Geschäftsbanken (d754).

Ergebnisse für den Zeitraum 1998-2000: ${ }^{2}$

Von hoher statistischer Signifikanz (zum 1-Prozent-Niveau) für die Determinierung der Zinsmarge ist das Verbot, Anleihen im Ausland zu tätigen (Variable d72). Ein Verbot der Beschaffung von Kapital im Ausland erhöht die Kosten der Banken. Diese werden durch eine entsprechende Zinsspreizung an die Kunden weitergegeben.

Ergebnisse für den Zeitraum 2001 - 2003:

In diesem Zeitraum ist keine der Liquiditätsvariablen mehr von statistischer Signifikanz. Diese Variablengruppe spielt für die Bestimmung der Höhe der Nettozinsmargen keine Rolle (mehr).

\section{Tab. 4-17: Signifikanz der Variablen „Liquidität“}

\begin{tabular}{l|l|c|c}
\multicolumn{2}{l|}{ Variable } & $1998-2000$ & $2001-2003$ \\
\hline $\begin{array}{l}\text { d71 } \\
\text { d72 }\end{array}$ & Keine verpflichtende Diversifizierung der Assets & Verbot fur Banken, Anleihen im Ausland zu tătigen & \\
d731 & Mindestliquidităt & \\
d74 & Hohe dieser Mindestliquidităt & \\
d753 & $\begin{array}{l}\text { Keine Reserven in Form auslăndischer Wăhrungen } \\
\text { d754 }\end{array}$ & $\begin{array}{l}\text { Anteil der in ausländischen Wăhrungen denominierten } \\
\text { Assets }\end{array}$ & \\
& \multicolumn{1}{|c|}{$X=$ Signifikanz, $(X)=$ schwache Signifikanz. }
\end{tabular}

Das Verbot, im Ausland Anleihen zu tätigen, scheint im Zeitablauf verstärkt aufgehoben worden zu sein: Die ehemals stark signifikante Variable verliert vollständig ihre Bedeutung für die Höhe der Zinsmargen. Insgesamt eher überraschend ist

1 Es wurde fur die Regression die Antwort , ja“ $=1$ und „nein" $=0$ gesetzt.

2 Fur diesen Zeitraum lagen Daten nur fur die Variablen a) bis d) vor. 
der Umstand, daß Liquiditätsbestimmungen keinen großen Einfluß auf die Zinsmargen nehmen. In diesem Punkt gelangten empirische Untersuchungen schon wiederholt $\mathrm{zu}$ widersprüchlichen Ergebnissen. ${ }^{1}$ Die Unterbindung finanzieller Beziehungen zum Ausland entspricht einer Abschottung des Inlandsmarktes. Damit kommt das Protektionspotential dieser Regulierung vollständig zum Tragen.

\subsubsection{Einlagensicherungssysteme}

Als Versicherung für die bei Banken hinterlegten Depositen dient das Einlagensicherungssystem der Vertrauensbildung und -sicherung in das Finanzsystem. Diese Reduktion von Unsicherheit sollte sich an niedrigen Zinsmargen ablesen lassen. Folgende Daten stehen für eine Regression zur Verfügung:

a) Existenz eines Einlagensicherungssystems (d81),

b) Finanziert durch Banken, Regierung oder beide ${ }^{2}$ (d82),

c) Kann die Einlagensicherungsbehörde in einer Bank intervenieren? (d85),

d) Anteil der Schulden, Wertpapiere etc., die weder implizit noch explizit durch ein Einlagensicherungssystem gedeckt sind ${ }^{3}(\mathrm{~d} 86)$,

e) Anteil der in den letzten 5 Jahren geschlossenen Banken (d87),

f) Vollständige Kompensation der Einleger beim letzten Bankzusammenbruch? (d88).

Ergebnisse für den Zeitraum 1998 - 2000:

Signifikant ist die Variable d86, d.h., daß der Anteil der nicht im Sicherungssystem erfaßten Elemente einen positiven Einfluß auf die Zinsmarge nimmt (5Prozent-Niveau). Je weniger Kredite oder Vermögenswerte durch das Sicherungssystem abgedeckt sind, um so höher das Verlustrisiko. Die Ausfallprämien steigen und dies treibt die Kosten für Kredite in die Höhe. Tendenziell steigen die Zinsmargen. Ähnliches gilt für die Einlagen: Je geringer der gewährte Schutz, um so größer das Risiko eines Verlustes der Depositen. Die Einleger verlangen eine Risikoprämie in Form höherer Habenzinsen.

\footnotetext{
Vgl. fur weitere Informationen Kalirajan et. al. (2000, 223).

2 Erfolgt die Finanzierung des Einlagensicherungssystems durch den Staat oder gemeinsam mit Banken, so wurde diese Antwort gleich 1 gesetzt. Erfolgt die Finanzierung allein durch Banken so nahm die Variable den Wert 0 an.

3 Der Anteil der nicht geschutzten Schulden, Wertpapiere etc. wird ins Verhältnis gesetzt zu den Assets.
} 
Ergebnisse für den Zeitraum 2001 - 2003:

Keine Variable ist statistisch signifikant.

Tab. 4-18: Schätzergebnisse - Einlagensicherungssysteme

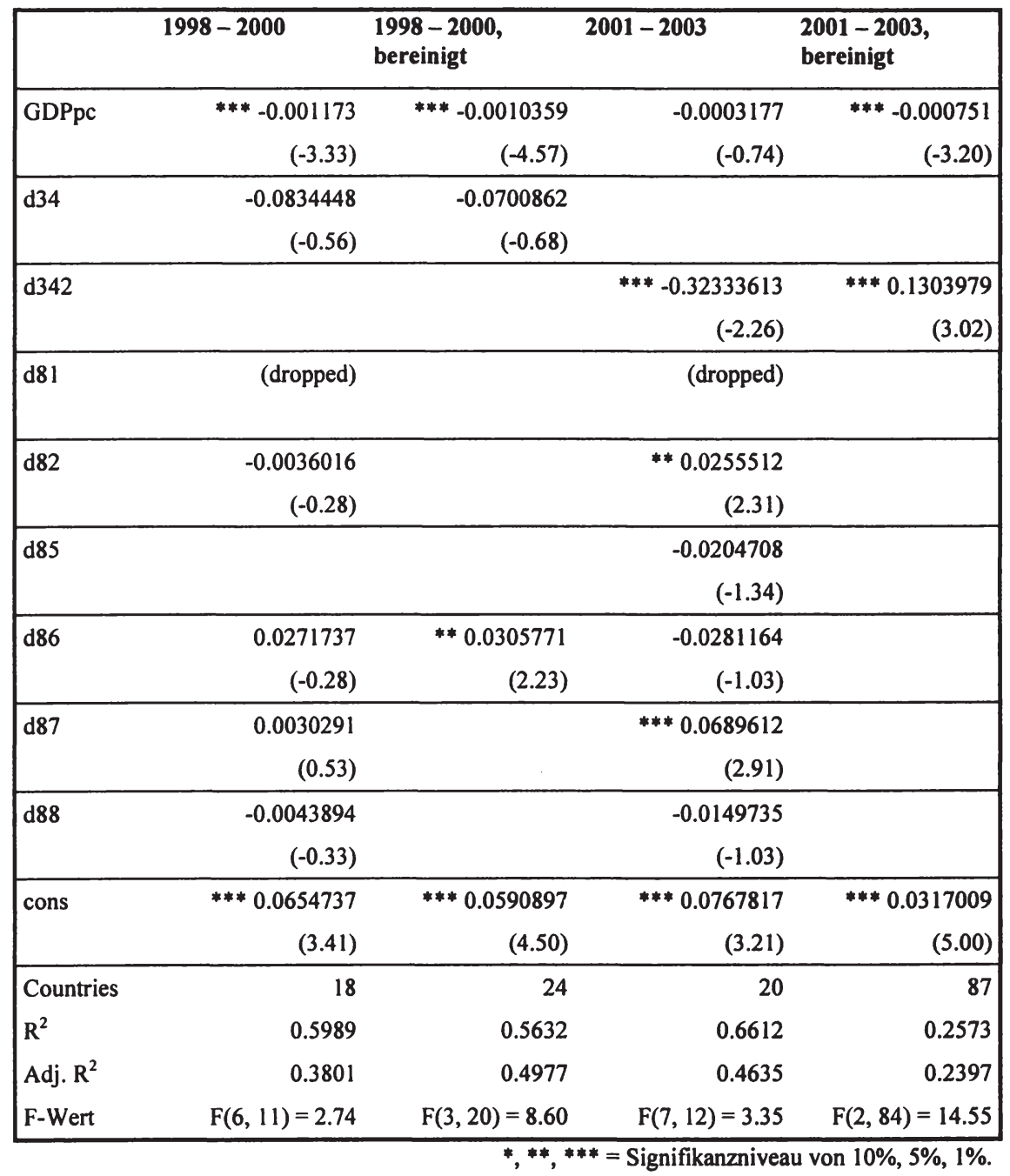


Tab. 4-19: Signifikanz der Variablen „Einlagensicherungssysteme“

\begin{tabular}{l|l|c|c}
\multicolumn{2}{l|}{ Variable } & $1998-2000$ & $2001-2003$ \\
\hline d81 & Existenz eines Einlagensicherungssystems & & \\
d82 & Finanziert durch Banken, Regierung oder beide & \\
d85 & Intervention der Einlagensicherungsbehorde in einer Bank & \\
d86 & Anteil nicht geschutzter Schulden / Wertpapiere & \\
d87 & Anteil der in den letzten 5 Jahren geschlossenen Banken & \\
d88 & Vollstăndige Kompensation der Einleger &
\end{tabular}

In der Regression dieser Variablengruppe ist der Signifikanzverlust der Variablen „Kapitalquote“ (d34) im Zeitraum 1998 bis 2000 auffallend. Eine Verfeinerung des obigen Ergebnisses stellen zwei weitere Regressionen dar. Die „Variante (1)“ liefert die Koeffizienten für eine Schätzung ohne die Kapitalquote (d34). In der „Variante (2)“ wurde mit Kapitalquote, aber unter Verzicht auf die Variable d86 geschätzt. Beide Abwandlungen liefern höhere F-Werte als die bereinigte Schätzung (12.99 und 19.56 statt 8.60). Dies verweist auf eine bessere Qualität der Schätzung.

Allerdings liefern die Bestimmtheitsmaße kein so eindeutiges Bild: Variante (1) weist ein leicht erhöhtes adjustiertes $R^{2}$ auf (Ansteigen von 0.4977 auf 0.5105 ). Dieser Wert verweist auf einen hohen Erklärungsgehalt der Variablen d86, denn die Grundschätzung ohne Variable d86 (Variante (2)) weist nur einen sehr geringen adjustierten $\mathrm{R}^{2}$-Wert von 0.3707 auf.

Insgesamt würde man zunächst von einer hohen Bedeutung des Einlagensicherungssystems ausgehen, weil es auf den leicht anfälligen Finanzmärkten die Unsicherheit auf Seiten der Sparer erheblich reduziert und das Vertrauen in das Finanzsystem stärkt. Entsprechend überraschend ist das Ergebnis der zunächst geringen bzw. später nicht mehr vorhandenen Signifikanz. Eine positive Interpretation lautet, daß nahezu alle Länder die Bedeutung eines funktionsfähigen Einlagensicherungssystems erkannt und dieses auch implementiert haben, so daß die Daten eine zu geringe Varianz aufweisen. Problematisch ist die sehr ausgedünnte Datenlage zu dieser Fragestellung. Mit nur 24 Angaben für die Variable d86 („Anteil der Schulden, Wertpapiere etc., die nicht vom Einlagensicherungssystem erfaßt werden"), sind kaum verläßliche Schlußfolgerungen aus den Schätzergebnissen zu ziehen. Entsprechend bietet die Variablengruppe „Einlagensicherungssysteme" keine wirtschaftspolitisch verwendbaren Ansätze, um in einem protektionistischen Sinne lenkend einzugreifen. 
Tab. 4-20: Schätzergebnisse - Einlagensicherungssysteme, ergänzend

\begin{tabular}{|c|c|c|c|}
\hline & $1998-2000$, bereinigt & $\begin{array}{l}1998-2000, \\
\text { Variante (1) }\end{array}$ & $\begin{array}{l}1998-2000, \\
\text { Variante (2) }\end{array}$ \\
\hline \multirow[t]{2}{*}{ GDPpc } & $* * *-0.0010359$ & $* * *-0.0010153$ & $* * *-0.0012724$ \\
\hline & $(-4.57)$ & $(-4.58)$ & $(-5.56)$ \\
\hline \multirow[t]{2}{*}{ d34 } & -0.0700862 & & **0.1012588 \\
\hline & $(-0.68)$ & & $(2.02)$ \\
\hline \multirow[t]{2}{*}{ d86 } & $* * 0.0305771$ & $* * 0.0268544$ & \\
\hline & (2.23) & $(2.17)$ & \\
\hline \multirow[t]{2}{*}{ cons } & $* * * 0.0590897$ & ***0.0509397 & $* * * 0.0470335$ \\
\hline & $(4.50)$ & (9.49) & $(5.90)$ \\
\hline Countries & 24 & 24 & 64 \\
\hline $\mathbf{R}^{2}$ & 0.5632 & 0.5530 & 0.3907 \\
\hline Adj. $R^{2}$ & 0.4977 & 0.5105 & 0.3707 \\
\hline F-Wert & $F(3,20)=8.60$ & $F(2,32)=12.99$ & $F(2,61)=19.56$ \\
\hline
\end{tabular}

\subsubsection{0 Öffentliches Monitoring / Offenlegungspflichten}

Neben den staatlichen Behörden beobachten und bewerten verschiedene institutionelle sowie private Vereinigungen wie auch die Presse die Finanzmärkte und ihre Akteure. Der Grad des Einflusses der öffentlichen Kontrolle von Banken auf die Zinsmargen wird gemessen anhand von Informationen zu:
a) Offenlegung der Off-Balance Sheets ${ }^{1}$ gegenüber Aufsichtsbehörden (d103),
b) Offenlegung der Off-Balance Sheets gegenüber der Öffentlichkeit (d104),
c) Publizierung der Methoden des Risikomanagements ${ }^{2}$ (d105),
d) Kann das Management der Banken für falsche Informationen juristisch zur Verantwortung gezogen werden? (d106),
e) Wurden Strafen für dieses Vergehen tatsächlich verhängt? (d107).

1 In den Off-Balance Sheets werden Aktiva und Passiva erfaßt, die nicht als reguläre Buchwerte Eingang in die Bilanz finden. Dabei handelt es sich oftmals um (gebuhrenpflichtige) Vorgänge, die gestundet werden oder in ihrer Realisation ungewiß sind (z.B. standby letter of credit, interest rate swaps, repurchase agreements etc.).

2

Fü die Regression wurde die Antwort „nein" = 1 gesetzt. 
Ergebnisse für den Zeitraum 1998 - 2000:

In der Regression ist nur die Variable d105 „Publizierung der Methoden des Risikomanagements" von schwacher Signifikanz mit positivem Vorzeichen zum 10-Prozent-Niveau. Je weniger ausgeprägt die verpflichtende Offenlegung des Risikomanagements ist, um so höher fällt die Zinsmarge aus. Dies läßt die vorsichtige Schlußfolgerung zu, daß eine ausgeprägte, öffentliche Kontrolle der Banken die Transparenz erhöht und eine höhere Effektivität erzwingt.

Tab. 4-21: Schätzergebnisse - Offenlegungspflicht

\begin{tabular}{|c|c|c|c|c|}
\hline & $1998-2000$ & $\begin{array}{l}1998-2000 \text {, } \\
\text { bereinigt }\end{array}$ & $2001-2003$ & $\begin{array}{l}2001-2003 \text {, } \\
\text { bereinigt }\end{array}$ \\
\hline \multirow[t]{2}{*}{ GDPpc } & $* * *-0.001199$ & $* * *-0.0011611$ & ** -0.0006864 & $* * *-0.000751$ \\
\hline & $(-4.55)$ & $(-4.86)$ & $(-2.52)$ & $(-3.20)$ \\
\hline \multirow[t]{2}{*}{ d34 } & 0.0866897 & $* * 0.1063811$ & & \\
\hline & $(1.52)$ & $(2.12)$ & & \\
\hline \multirow[t]{2}{*}{ d342 } & & & $* * * 0.1242258$ & *** 0.1303979 \\
\hline & & & (2.71) & $(3.02)$ \\
\hline d 103 & (dropped) & & (dropped) & \\
\hline \multirow[t]{2}{*}{ d104 } & 0.0055283 & & & \\
\hline & $(0.69)$ & & & \\
\hline \multirow[t]{2}{*}{ d 105} & * 0.0126569 & $* 0.0103404$ & 0.0050184 & \\
\hline & $(1.85)$ & $(1.86)$ & $(0.80)$ & \\
\hline \multirow[t]{2}{*}{ d 106} & 0.0005139 & & (dropped) & \\
\hline & $(0.04)$ & & & \\
\hline \multirow[t]{2}{*}{ d107 } & -0.0075395 & & -0.0006972 & \\
\hline & $(-1.17)$ & & $(-0.12)$ & \\
\hline \multirow[t]{2}{*}{ cons } & $* * * 0.0441414$ & $* * * 0.0385658$ & $* * * 0.0291936$ & ***0.0317009 \\
\hline & $(4.07)$ & $(4.20)$ & (3.07) & $(5.00)$ \\
\hline Countries & 54 & 62 & 83 & 87 \\
\hline $\mathrm{R}^{2}$ & 0.4487 & 0.4173 & 0.2538 & 0.2573 \\
\hline Adj. $R^{2}$ & 0.3783 & 0.3872 & 0.2155 & 0.2397 \\
\hline F-Wert & $F(6,47)=6.38$ & $F(3,58)=13.85$ & $F(4,78)=6.63$ & $F(2,84)=14.55$ \\
\hline
\end{tabular}

Ergebnisse für den Zeitraum 2001 - 2003:

Keine Variable ist statistisch signifikant. 
Diese Ergebnisse lassen zwei Interpretationen zu: Die öffentliche Beobachtung der Bankenmärkte ist bereits so stark über alle Länder hinweg ausgeprägt, daß dieser Effekt bereits in den Zinsmargenhöhen enthalten ist. Oder aber es handelt sich um eher "weiche“ Faktoren, die keinen Niederschlag in der Zinsmarge finden. Letztere Interpretation ist wahrscheinlicher.

\section{Tab. 4-22: Signifikanz der Variablen „Offenlegungspflicht“}

\begin{tabular}{l|l|c|c}
\multicolumn{2}{l|}{ Variable } & $1998-2000$ & $2001-2003$ \\
\hline d103 & Off-Balance-Sheets - Aufsichtsbehorden & \\
d104 & Off-Balance-Sheets - Öfentlichkeit & $(X)$ & \\
d105 & Keine Publizierung der Risikomanagementmethoden & \\
d106 & Juristische Verantwortung des Managements & \\
d107 & Tatsăchliche Verhăngung von Strafen $\quad X=$ Signifikanz, (X) = schwache Signifikanz.
\end{tabular}

\subsubsection{Haftbarkeit bzw. juristische Belangbarkeit des Bankmanagements}

Die Eingriffsmöglichkeiten der Aufsichtsbehörden in die Bankengeschäfte und die Belangbarkeit des Bankmanagements für Verfehlungen wird nun untersucht. Diese Faktoren beeinflussen das Verhalten des Managements. Inwieweit eine Bank bzw. ihr Management juristisch für Fehlverhalten belangt werden kann, messen folgende Daten:'

a) Existieren Mechanismen in Form von Unterlassungsanordnungen, die automatisch zu zivil- oder strafrechtlichen Sanktionen fuhren? (d111)

b) Kann die Aufsichtsbehörde die Bildung von Rückstellungen für anfallende oder potentielle Verluste anordnen? (d112)

c) Kann die Aufsichtsbehörde Beschlüsse des Managements aufheben betreffend: Dividendenausschüttung? (d113), Bonuszahlungen? (d114), Gebührenhöhe? (d115)

d) Wurden derartige Eingriffe seitens der Aufsichtsbehörde in den letzten 5 Jahren unternommen? (d116)

e) Kann die Aufsichtsbehörde Rechte der Gesellschafter/Aktieninhaber aussetzen und die Insolvenz einer Bank erklären? (d117)

1 Alle Antworten wurden folgendermaßen umgesetzt: ,ja“ $=0$, ,nein“ $=1$. 
f) Kann die Aufsichtsbehörde einige oder alle Eigentümerrechte aussetzen für eine in finanzielle Schwierigkeiten geratene Bank? (d118)

g) Existieren Mindestvorgaben für die Solvenz, bei deren Unterschreitung automatisch Interventionen erfolgen? (d119)

Ergebnisse für den Zeitraum 1998 - 2000:

Mit einem positiven Vorzeichen und nur schwach signifikant zum 10-ProzentNiveau geht die Variable d116 „Aufhebung von Entscheidungen des Managements seitens der Aufsichtbehörde in den letzten 5 Jahren" in die Regression ein. Je weniger die Aufsichtsbehörde von diesem Recht Gebrauch macht, um so höher sind die Zinsmargen. Dies erlaubt die Interpretation, daß eine eher durchsetzungsschwache Aufsichtsbehörde dazu führt, daß die Banken die Zinsmargen zu eigenen Gunsten heraufsetzen.

Die Variable d118 („Aussetzen der Eigentümerrechte durch die Aufsichtsbehörde") weist in der Regression ein negatives Vorzeichen auf und geht ebenfalls nur schwach signifikant zum 5-Prozent-Niveau ein. Eingriffe in die Eigentumsrechte stellen faktische Enteignungen dar. Je weniger die Aufsichtsbehörden Eigentümerrechte aussetzen können, um so niedriger sind die Zinsmargen. Eine mögliche Interpretation dieses Ergebnisses ist darin zu sehen, daß willkürliches Verhalten von Zentralbanken und Aufsichtsbehörden zulasten der Kreditinstitute unterbunden wird. Andererseits können finanzielle Schwierigkeiten einer Bank im sensiblen Finanzwesen schnell um sich greifen und auch andere Institute erfassen. Ein weitreichendes Eingriffsrecht der Aufsichtsbehörden könnte also der Krisenbekämpfung dienen. Dieses Schätzergebnis ist also nicht eindeutig zu interpretieren.

Ergebnisse für den Zeitraum 2001 - 2003:

Keine Variable weist einen signifikanten Wert auf.

Die noch im ersten Zeitraum zumindest schwach signifikanten Variablen haben an Relevanz verloren; die Ausgestaltung der Haftbarkeit scheint keinen Einfluß auf die Zinsmargen zu nehmen. Entweder liegen in den Ländern mittlerweile ähnliche Regeln und dadurch nur eine sehr geringe Varianz der Daten vor oder aber sie haben generell keinen Einfluß mehr auf die Höhe der Zinsmargen. Damit kann die Wirtschaftspolitik in diesem Bereich keine aktive Steuerung der Zinsmargen vornehmen. 
Tab. 4-23: Schätzergebnisse - Haftbarkeit Bankmanagement

\begin{tabular}{|c|c|c|c|c|}
\hline & $1998-2000$ & $\begin{array}{l}1998-2000 \text {, } \\
\text { bereinigt }\end{array}$ & $2001-2003$ & $\begin{array}{l}2001-2003 \text {, } \\
\text { bereinigt }\end{array}$ \\
\hline \multirow[t]{2}{*}{ GDPpc } & $* * *-0.0011105$ & $* * *-0.001243$ & $* *-0.0007173$ & $* * *-0.000751$ \\
\hline & $(-4.46)$ & $(-5.24)$ & $(-2.53)$ & $(-3.20)$ \\
\hline \multirow[t]{2}{*}{$\mathrm{d} 34$} & 0.0880085 & ****0.1463107 & & \\
\hline & $(0.174)$ & $(2.73)$ & & \\
\hline \multirow[t]{2}{*}{$\mathrm{d} 342$} & & & $* * * 0.1582064$ & $* * * 0.1303979$ \\
\hline & & & (3.09) & (3.02) \\
\hline \multirow[t]{2}{*}{ d111 } & -0.0012677 & & $* * 0.0150444$ & \\
\hline & $(-0.19)$ & & (2.16) & \\
\hline \multirow[t]{2}{*}{ d 112} & -0.0060311 & & -0.0024318 & \\
\hline & $(-0.68)$ & & $(-0.21)$ & \\
\hline \multirow[t]{2}{*}{ d 113} & 0.0059924 & & 0.005415 & \\
\hline & $(0.57)$ & & $(0.57)$ & \\
\hline \multirow[t]{2}{*}{ d 114} & -0.0155469 & & -0.0116663 & \\
\hline & $(-1.00)$ & & $(-1.01)$ & \\
\hline \multirow[t]{2}{*}{ d 115} & 0.0165876 & & 0.0037977 & \\
\hline & $(1.17)$ & & $(0.35)$ & \\
\hline \multirow[t]{2}{*}{ d1 16} & 0.0115299 & $* 0.0103779$ & 0.0032679 & \\
\hline & $(1.51)$ & $(1.90)$ & $(0.41)$ & \\
\hline \multirow[t]{2}{*}{ d117 } & -0.0075047 & & -0.0051255 & \\
\hline & $(-0.97)$ & & $(-0.58)$ & \\
\hline \multirow[t]{2}{*}{ d 118} & -0.0114874 & $* *-0.0138316$ & 0.0073254 & \\
\hline & $(-1.37)$ & $(-1.95)$ & $(0.77)$ & \\
\hline \multirow[t]{2}{*}{ d119 } & -0.0064417 & & -0.0072429 & \\
\hline & $(-0.87)$ & & $(-1.03)$ & \\
\hline \multirow[t]{2}{*}{ cons } & $* * 0.0483694$ & ****0.0384173 & $* * * 0.0269626$ & $* * * 0.0317009$ \\
\hline & $(4.57)$ & $(4.32)$ & (3.16) & $(5.00)$ \\
\hline Countries & 56 & 58 & 75 & 87 \\
\hline $\mathrm{R}^{2}$ & 0.5107 & 0.4483 & 0.3424 & 0.2573 \\
\hline Adj. $R^{2}$ & 0.3884 & 0.4066 & 0.2275 & 0.2397 \\
\hline F-Wert & $F(11,44)=4.18$ & $F(4,53)=10.77$ & $F(11,63)=2.98$ & $F(2,84)=14.55$ \\
\hline
\end{tabular}


Tab. 4-24: Signifikanz der Variablen „Haftbarkeit“

\begin{tabular}{|c|c|c|c|}
\hline Varia & & $1998-2000$ & $2001-2003$ \\
\hline d111 & Mechanismen furr zivil- oder strafrechtliche Sanktionen & & \\
\hline d 112 & Anordnung von Ruckstellungen & & \\
\hline d113 & ...Dividendenausschuttung verhindert & & \\
\hline d1 14 & ...Bonuszahlungen verhindert & & \\
\hline d1 15 & ...Gebuhrenerhőhung verhindert & & \\
\hline d1 16 & $\begin{array}{l}\text { Aufhebung von Managementbeschlüssen in den letzten } \\
5 \text { Jahren? }\end{array}$ & $(\mathrm{X})$ & \\
\hline d 117 & Insolvenz einer Bank erklăren & & \\
\hline d1 18 & Eigentumerrechte aussetzen & $(\mathrm{X})$ & \\
\hline d119 & $\begin{array}{l}\text { Mindestvorgaben fur Solvenz: automatische } \\
\text { Interventionen }\end{array}$ & & \\
\hline
\end{tabular}

\subsubsection{Kontrollintensität}

Regelmäßige Überprüfungen der Banken senken das Betrugsrisiko. Sie stellen somit eine Schutzfunktion der Bankkunden wie auch der Geschäftspartner dar. Kontrollen stärken das Vertrauen der Bankkunden in die Finanzinstitutionen. Dies wirkt sich senkend auf die Zinsmargen aus, da einerseits die Risikoprämien der Anleger geringer ausfallen und andererseits die Stabilität des Systems erhöht und die Gefahr eines Bankzusammenbruchs minimiert wird. Inwieweit die intensive Kontrolltätigkeit der Aufsichtsbehörden eine Rolle für die Zinsmargen spielt, wird mit Hilfe folgender Daten geschätzt:

a) Existiert mehr als eine Aufsichtsbehörde? (d121),

b) Gesamtzahl der bankenüberwachenden Mitarbeiter in der Aufsichtsbehörde (d122),

c) Anzahl der Vor-Ort-Prüfungen pro Bank in den letzten 5 Jahren (d124),

d) Wie häufig werden mittlere und kleinere Banken überprüft?' (d125),

e) Durchschnittliche Betriebszugehörigkeit der Prüfer (Berufserfahrung in Jahren) (d126),

f) Müssen alle vom Prüfer ermittelten Verstöße gemeldet werden? (d128),

1 Jăhrliche Kontrollen = 1, Kontrollen alle 2 Jahre oder im großeren Abstand $=0$. 

g) Existieren vorgeschriebene Verhaltensweisen in einem solchen Fall? (d129),
h) Sind die Prüfer für ihr Verhalten rechtlich zu belangen? (d1210).

Ergebnisse für den Zeitraum 1998 - 2000:

Nur die Variable d126 „Berufserfahrung der überwachenden Mitarbeiter“ ist zum 5-Prozent-Niveau mit negativem Vorzeichen signifikant. Dies bedeutet, je erfahrener die Mitarbeiter der Aufsichtsbehörden sind, um so niedriger die Zinsmargen. Berufserfahrung scheint sich in effektiven Kontrollen niederzuschlagen.

Ergebnisse für den Zeitraum 2001 - 2003:

Als stark signifikante, mit positivem Vorzeichen versehene Variable geht d124 „Anzahl der Vor-Ort-Prüfungen pro Bank“ zum 1-Prozent-Niveau in die Regression ein. Variable d125 "Überprüfungshäufigkeit kleinerer und mittlerer Banken" geht ebenfalls positiv, aber nur schwach signifikant in die Schätzung ein. Regelmäßige intensive Vor-Ort-Kontrollen erweisen sich also als förderlich für eine niedrige Zinsmarge.

Tab. 4-25: Schätzergebnisse - Kontrollintensität

\begin{tabular}{|c|c|c|c|c|}
\hline & $1998-2000$ & $\begin{array}{l}1998-2000 \text {, } \\
\text { bereinigt }\end{array}$ & $2001-2003$ & $\begin{array}{l}2001-2003 \text {, } \\
\text { bereinigt }\end{array}$ \\
\hline \multirow[t]{2}{*}{ GDPpc } & $* * *-0.0014217$ & $* * *-0.0012939$ & ${ }^{* * *}-0.000957$ & $* * *-0.0007377$ \\
\hline & $(-2.76)$ & $(-4.20)$ & $(-2.86)$ & $(-2.82)$ \\
\hline \multirow[t]{2}{*}{$\mathrm{d} 34$} & 0.0355803 & 0.0863679 & & \\
\hline & $(0.33)$ & (1.53) & & \\
\hline \multirow[t]{2}{*}{ d342 } & & & *** 0.1546393 & ${ }^{* * *} 0.1554419$ \\
\hline & & & (3.37) & (3.93) \\
\hline \multirow[t]{2}{*}{ d 121} & -0.001655 & & 0.0071719 & \\
\hline & $(-0.13)$ & & $(0.96)$ & \\
\hline \multirow[t]{2}{*}{ d 122} & -0.000133 & & -0.000012 & \\
\hline & $(-0.05)$ & & $(-0.62)$ & \\
\hline \multirow[t]{2}{*}{ d 123} & -0.0021124 & & & \\
\hline & $(-1.47)$ & & & \\
\hline \multirow[t]{2}{*}{ d124 } & -0.0012654 & & *** 0.0004403 & $* * * 0.0004104$ \\
\hline & $(-0.68)$ & & $(4.70)$ & $(5.01)$ \\
\hline
\end{tabular}


Tab. 4-25: Schätzergebnisse - Kontrollintensität (Forts.)

\begin{tabular}{|c|c|c|c|c|}
\hline & $1998-2000$ & $\begin{array}{l}\text { 1998-2000, } \\
\text { bereinigt }\end{array}$ & $2001-2003$ & $\begin{array}{l}2001-2003, \\
\text { bereinigt }\end{array}$ \\
\hline \multirow[t]{2}{*}{ d 125} & 0.0018207 & & 0.0082362 & $* 0.0094538$ \\
\hline & $(0.22)$ & & $(1.35)$ & (1.93) \\
\hline \multirow[t]{2}{*}{ d 126} & -0.0012036 & $* *-0.0013687$ & 0.0000080 & \\
\hline & $(-1.37)$ & $(-2.17)$ & $(-0.01)$ & \\
\hline \multirow[t]{2}{*}{ d127 } & 0.0020082 & & & \\
\hline & $(0.20)$ & & & \\
\hline d 128 & (dropped) & & & \\
\hline \multirow[t]{2}{*}{ d 129} & -0.0092451 & & 0.0079636 & \\
\hline & $(-0.86)$ & & (1.08) & \\
\hline \multirow[t]{2}{*}{ d 1210} & 0.0020971 & & 0.0022927 & \\
\hline & $(0.24)$ & & $(0.39)$ & \\
\hline \multirow[t]{2}{*}{ Cons } & $* * * 0.0776102$ & $* * * 0.0601863$ & 0.0162802 & $* * * 0.0203186$ \\
\hline & (3.34) & $(5.53)$ & $(1.35)$ & (3.36) \\
\hline Countries & 38 & 46 & 66 & 76 \\
\hline $\mathrm{R}^{2}$ & 0.4245 & 0.4022 & 0.5322 & 0.5067 \\
\hline Adj. $R^{2}$ & 0.1810 & 0.3595 & 0.4571 & 0.4789 \\
\hline F-Wert & $F(11,26)=1.74$ & $F(3,42)=9.42$ & $F(9,56)=7.08$ & $F(4,71)=18.23$ \\
\hline
\end{tabular}

Tab. 4-26: Signifikanz der Variablen „Kontrollintensität“

\begin{tabular}{l|l|c|c}
\multicolumn{2}{l|}{ Variable } & $1998-2000$ & $2001-2003$ \\
\hline d121 & Mehr als eine Aufsichtsbehörde & & \\
d122 & Gesamtzahl der bankenuberwachenden Mitarbeiter & \\
d124 & Anzahl der Vor-Ort-Prufungen pro Bank & X \\
d125 & Prufungshăufigkeit kleinerer und mittlerer Banken & $(\mathrm{X})$ \\
d126 & Durchschnittliche Betriebszugehörigkeit der Prufer & \\
d128 & Meldepflicht bei Verstoßen & \\
d129 & Vorgeschriebene Verhaltensweisen bei & \\
d1210 & Serstoßentdeckung & & \\
& Sind Prufer rechtlich zu belangen? & $X=$ Signifikanz, (X) = schwache Signifikanz.
\end{tabular}


In diesem Fall liegt eine Korrelation zwischen der „Kapitalquote“ (d34) und der Variablen „Berufserfahrung der Prüfer“ (d126) vor.

Tab. 4-27: Korrelationsmatrix

\begin{tabular}{l|ll} 
& $\mathrm{d} 34$ & $\mathrm{~d} 126$ \\
\hline $\mathrm{d} 34$ & 1.0000 & \\
$\mathrm{~d} 126$ & $-0,1763$ & 1.0000
\end{tabular}

Die Korrelation ist zwar gering, aber dennoch von großem Einfluß hinsichtlich der Bedeutung der Kapitalquote für die Regression. Diese wird insignifikant, wenn $\mathrm{d} 126$ in der Schätzgleichung aufgenommen wird, wie folgende Tabelle verdeutlicht:

Tab. 4-28: Schätzergebnisse - Kontrollintensität, ergänzend

\begin{tabular}{|c|c|c|c|}
\hline & $\begin{array}{l}\text { 1998-2000, } \\
\text { bereinigt }\end{array}$ & $\begin{array}{l}1998-2000, \\
\text { Variante (1) }\end{array}$ & $\begin{array}{l}1998-2000, \\
\text { Variante (2) }\end{array}$ \\
\hline \multirow[t]{2}{*}{ GDPpc } & $* * *-0.0012939$ & $* * *-0,0013699$ & $* * *-0.0012724$ \\
\hline & $(-4.20)$ & $-4,38$ & $(-5.56)$ \\
\hline \multirow[t]{2}{*}{ d34 } & 0.0863679 & & ** 0.1012588 \\
\hline & (1.53) & & $(2.02)$ \\
\hline \multirow[t]{2}{*}{ d126 } & ** -0.0013687 & ${ }^{* *}-0,0015674$ & \\
\hline & $(-2.17)$ & $-2,45$ & \\
\hline \multirow[t]{2}{*}{ Cons } & ${ }^{* * *} 0.0601863$ & $* * * 0,0744006$ & $* * 0.0470335$ \\
\hline & (5.53) & 11,73 & (5.90) \\
\hline Countries & 46 & 49 & 64 \\
\hline $\mathrm{R}^{2}$ & 0.4022 & 0,3635 & 0.3907 \\
\hline Adj. $R^{2}$ & 0.3595 & 0,3359 & 0.3707 \\
\hline F-Wert & $F(3,42)=9.42$ & $F(2,46)=13.14$ & $F(2,61)=19.56$ \\
\hline
\end{tabular}

Das adjustierte Bestimmtheitsmaß sinkt im Vergleich zur bereinigten Schätzung. Der F-Wert steigt erheblich.

Gut ausgebildete Prüfinstanzen und häufige Kontrollen erweisen sich folglich als förderlich für ein gut funktionierendes und vertrauenswürdiges Finanzsystem; die Zinsmargen dieser Länder sind tendenziell geringer. Die Wirtschaftspolitik muß sich folglich um effektive Kontrollen bemühen, um niedrige Zinsmargen zu erreichen. 
Ein Problem in diesem Zusammenhang kann der diskriminierende Umgang der Behörden mit ausländischen Banken darstellen. Für ein solches Verhalten gibt es, wie im obigen Kapitel bereits ausgeführt, einige Hinweise (Feketekuty 1988). Durch diskriminierend agierende Aufsichtsbehörden können ausländische Konkurrenten erheblich geschädigt und in ihrer Geschäftstätigkeit behindert werden. Dieser inoffizielle Bereich liefert einer protektionistisch agierenden Wirtschaftspolitik reichlich Ansätze, um ihre Ziele zu verfolgen.

\subsubsection{Economic Freedom Indicator}

Zur Einschätzung der ökonomischen und sozialen Rahmenbedingungen eines Landes wird der Economic Freedom Indicator verwendet. Die einzelnen Regulierungen werden gemäß eines Punktesystem bewertet. Je geringer die erreichte Punktzahl eines Landes, desto mehr ökonomische Freiheiten genießen die Wirtschaftssubjekte. Getestet werden:

a) Economic Freedom Indicator: Gesamtindikator,

b) Staatliche Interventionen (Teilindikator),

c) Regulierungen (Teilindikator).

Ergebnisse für den Zeitraum 1998 - 2000:

In diesem Zeitraum ist nur der Gesamtindikator zum 5-Prozent-Niveau mit positivem Vorzeichen signifikant. Steigt der Gesamtindikator, so steigt auch die Zinsmarge. Nimmt die ökonomische Freiheit in einem Land ab, d.h. der Regulierungsgrad steigt oder es treten zunehmend Beschränkungen wirtschaftlicher Aktivitäten auf, so divergieren die Zinsen zunehmend.

Ergänzend wurden anschließend auch die Indikatoren Geldpolitik (positives Vorzeichen, Signifikanz zum 1-Prozent-Niveau) sowie Löhne und Preise (negatives Vorzeichen, zum 5-Prozent-Niveau) signifikant getestet.'

Ergebnisse für den Zeitraum 2001 - 2003:

Alle drei Variablen sind insignifikant.

Je größer das Ausmaß an ökonomischer Freiheit, um so niedriger waren die Zinsmargen der Länder. Dieser Indikator hat aber für den jüngeren Zeitraum vollständig an Bedeutung verloren.

Ergebnisse dieser Schătzung, siehe Anhang A4. 
Tab. 4-29: Schätzergebnisse - Economic Freedom Indicator

\begin{tabular}{|c|c|c|c|c|}
\hline & $1998-2000$ & $\begin{array}{l}1998-2000 \text {, } \\
\text { bereinigt }\end{array}$ & $2001-2003$ & $\begin{array}{l}2001-2003 \text {, } \\
\text { bereinigt }\end{array}$ \\
\hline \multirow[t]{2}{*}{ GDPpc } & $* *-0.0006851$ & $* *-0.0007453$ & $* * *-0.0007648$ & $* * *-0.000751$ \\
\hline & $(-2.10)$ & $(-2.31)$ & $(-3.30)$ & $(-3.20)$ \\
\hline \multirow[t]{2}{*}{ d34 } & **0.1168417 & ** 0.980208 & & \\
\hline & (2.23) & $(2.05)$ & & \\
\hline \multirow[t]{2}{*}{ d342 } & & & *** 0.1474381 & **** 0.1303979 \\
\hline & & & (4.14) & (3.02) \\
\hline Economic & $* 0.0164253$ & ** 0.122967 & * 0.0113999 & \\
\hline Freedom & (1.94) & $(0.41)$ & $(1.68)$ & \\
\hline Govern. & -0.0047704 & & -0.0017223 & \\
\hline Intervention & $(-1.32)$ & & $(-0.55)$ & \\
\hline \multirow[t]{2}{*}{ Regulation } & -0.0010475 & & *-009164 & \\
\hline & $(-0.20)$ & & $(-1.73)$ & \\
\hline \multirow[t]{2}{*}{ Cons } & 0.0104876 & 0.0082248 & ** 0.0290895 & ${ }^{* * *} 0.0317009$ \\
\hline & $(0.52)$ & $(0.41)$ & $(2.64)$ & $(5.00)$ \\
\hline Countries & 63 & 63 & 60 & 87 \\
\hline $\mathrm{R}^{2}$ & 0.4448 & 0.4277 & 0.4170 & 0.2573 \\
\hline Adj. $R^{2}$ & 0.3961 & 0.3986 & 0.3630 & 0.2397 \\
\hline F-Wert & $F(5,57)=9.13$ & $F(3,59)=14.70$ & $F(5,54)=7.72$ & $F(2,84)=14.55$ \\
\hline
\end{tabular}

Tab. 4-30: Signifikanz der Variablen „Economic Freedom Indicator“

Variable

Economic Freedom (Gesamtindikator)

Governmental Intervention (Teilindikator)

Regulation (Teilindikator)

\begin{tabular}{l|c|c} 
& $1998-2000$ & $2001-2003$ \\
& $X$ & \\
$X=$ Signifikanz, $(X)=$ schwache Signifikanz.
\end{tabular}

\subsubsection{Nicht oder kaum signifikante Aspekte}

Eine schwache Signifikanz der Variablen für die beiden untersuchten Zeiträume weisen folgende Schätzungen auf: 


\section{Ratings}

Die ständige Beobachtung und Bewertung der Marktteilnehmer durch internationale Rating-Agenturen sollte die Transparenz erhöhen und Informationsasymmetrien abbauen. Dieser Umstand sollte sich in niedrigeren Zinsmargen widerspiegeln, da die guten Rating-Wertungen als Qualitätsmerkmal gelten.

In dieser Variablengruppe standen folgende Daten zur Verfügung:

a) Anteil international bewerteter Banken (z.B. Moody's, Standard and Poor) (d109),

b) Rating von Wertpapieremissionen (d1101),

c) Rating des Handels kurzfristiger Geldmarktpapiere (d1102) oder

d) Rating anderer Geschäftstätigkeiten (Pensionsfonds, Versicherungen etc.) (d1103).

Tab. 4-31: Schätzergebnisse - Ratings

\begin{tabular}{|c|c|c|c|c|}
\hline & $1998-2000$ & $\begin{array}{l}1998-2000 \text {, } \\
\text { bereinigt }\end{array}$ & $2001-2003$ & $\begin{array}{l}2001-2003, \\
\text { bereinigt }\end{array}$ \\
\hline \multirow[t]{2}{*}{ GDPpc } & $* * *-0.0009554$ & $* * *-0.0011084$ & $* *-0.0007489$ & $* * *-0.000751$ \\
\hline & $(-3.66)$ & $(-4.29)$ & $(-2.47)$ & $(-3.20)$ \\
\hline \multirow[t]{2}{*}{ d34 } & 0.090655 & * 0.0947714 & & \\
\hline & (1.64) & $(1.76)$ & & \\
\hline \multirow[t]{2}{*}{ d342 } & & & ***0.1428634 & $* * * 0.1303979$ \\
\hline & & & (2.59) & $(3.02)$ \\
\hline \multirow[t]{2}{*}{ d109 } & -0.0086871 & -0.0150501 & 0.0091932 & \\
\hline & $(-0.81)$ & $(-1.76)$ & $(0.77)$ & \\
\hline \multirow[t]{2}{*}{ dl101 } & 0.0058351 & & 0.0153961 & \\
\hline & $(0.53)$ & & $(1.33)$ & \\
\hline \multirow[t]{2}{*}{ d1 102} & -0.0046094 & & -0.0094381 & \\
\hline & $(-0.49)$ & & $(-0.93)$ & \\
\hline \multirow[t]{2}{*}{ d1 103} & 0.0008175 & & 0.0003548 & \\
\hline & $(0.13)$ & & $(0.04)$ & \\
\hline \multirow[t]{2}{*}{ Cons } & $* * * 0.0477603$ & $* * * 0.0548626$ & 0.0248146 & $* * * 0.0317009$ \\
\hline & (3.89) & $(5.41)$ & $(1.78)$ & $(5.00)$ \\
\hline
\end{tabular}


Tab. 4-31: Schätzergebnisse - Ratings (Forts.)

\begin{tabular}{|c|c|c|c|c|}
\hline & $1998-2000$ & $\begin{array}{l}1998-2000, \\
\text { bereinigt }\end{array}$ & $2001-2003$ & $\begin{array}{l}2001-2003 \text {, } \\
\text { bereinigt }\end{array}$ \\
\hline Countries & 41 & 50 & 66 & 87 \\
\hline $\mathrm{R}^{2}$ & 0.4922 & 0.4631 & 0.3037 & 0.2573 \\
\hline Adj. $R^{2}$ & 0.4026 & 0.4281 & 0.2329 & 0.2397 \\
\hline F-Wert & $F(6,34)=5.49$ & $F(3,46)=13.23$ & $F(6,59)=4.29$ & $F(2,84)=14.55$ \\
\hline
\end{tabular}

Nur die internationale Bewertung von Banken ist von schwacher Signifikanz im Zeitraum 1998 - 2000, mit einem negativen Vorzeichen zum 10-Prozent-Niveau. Je höher der Anteil der Banken eines Landes, die in Ratings erfaßt werden, um so niedriger die Zinsmargen - ein schlüssiger Zusammenhang, da internationale Ratings nur für gewichtige, international agierende Banken vollzogen werden, die einem entsprechenden Wettbewerbsdruck ausgesetzt sind. Allerdings rät die schwache Signifikanz zur äußersten Vorsicht in der Interpretation dieses Ergebnisses, insbesondere hinsichtlich der Formulierung wirtschaftspolitischer Empfehlungen.

Keinerlei Signifikanz weisen die folgenden Variablen auf:

\section{Einfluß von staatlichen und ausländischen Vermögensanteilen bei Banken}

Ein hoher Anteil staatlichen Besitzes am Bankenkapital deutet gemeinhin auf eine starke staatliche Präsenz im Bankenmarkt und ist ein Hinweis auf eine relativ stark dirigistisch geprägte Wirtschaftspolitik. Je nach wirtschaftspolitischer Vorstellung der Regierungen, könnte direkter Einfluß auf die Höhe der Zinsen genommen werden. Damit stünde den Entscheidungsträgern ein weiteres wirtschaftspolitisches Instrument zur Verfügung. Die freie Preisbildung, in diesem Fall die Zinsfindung, wäre eingeschränkt und der Erreichung wirtschaftspolitischer Ziele untergeordnet. Entsprechend vermutete die Autorin ein höheres Zinsniveau in solchen Ländern, in denen ein relativ hohes Ausma $\beta$ an staatlichem Einfluß im Bankensektor vorherrscht. Ebenfalls lag es nahe zu vermuten, daß ein Bankenmarkt, der sich zu großen Teilen im Besitz von Ausländern befindet, deren Zinssätze importiert. Inwieweit staatlicher oder ausländischer Besitz von Vermögenswerten der Banken die Zinsmargen beeinflußt, wird mit folgenden Aspekten gemessen:

a) Anteil des Bankenvermögens (Assets) in staatlicher Hand [mehr als 50 v.H.] (d37) und

b) Anteil des Bankenvermögens (Assets) in ausländischer Hand [mehr als 50 v.H.] (d38). 
Es wurde keinerlei Signifikanz dieser Variablen ermittelt. Die Vermutung, daß Märkte mit einer starken staatlichen Präsenz höhere Zinsmargen aufweisen, läßt sich statistisch nicht belegen. Ebenfalls keinen Einfluß auf die Zinsgestaltung hat der Besitz von Bankvermögen durch Ausländer.

\section{Allgemeine Befugnisse der Aufsichtsbehörden}

$\mathrm{Ob}$ weitreichende direkte Zugriffsrechte der Aufsichtsbehörden gegenüber den Banken von Bedeutung sind für die Höhe der Zinsmargen, wird anhand folgender Variablen getestet:

a) Aufsichtsbehörde kann interne Organisationsstrukturen verändern (d61),

b) Wurde dieses Recht in den letzten 5 Jahren ausgeübt? (d62),

c) Aussetzen der Shareholder-Rechte (d1110),

d) Ablösung und Ersatz des Managements (d1111),

e) Ablösung und Ersatz der Direktoren (d1112) und

f) Verzicht auf Anwendung einiger Regulierungen (d1113).

Diese direkten Eingriffsrechte der Aufsichtsbehörden spielen keine Rolle für die Höhe der Zinsmargen.

\section{Geschlossene bzw. fusionierte Banken}

Untersucht werden die zwei Fragestellungen:

a) Anteil der geschlossenen Banken in den letzten 5 Jahren (d1115) und

b) Anteil fusionierter Banken an Gesamtbanken (d87).

$\mathrm{Zu}$ vermuten wäre, daß ein hoher Anteil fusionierter Banken Anzeichen für einen hohen Wettbewerbsdruck ist, der in eine steigende Konzentration auf dem Bankenmarkt mündet. Ein hoher Anteil geschlossener Banken kann Hinweis auf eine durchsetzungsstarke Aufsichtsbehörde sein, die ihre Bestimmungen nicht erfültt sieht. Empirisch läßt sich jedoch nicht belegen, daß sich diese Aspekte auf die Zinsmargen auswirken.

Damit sind wir nun am Ende dieser Analysen des Zusammenhangs von Zinsmargen und den einzelnen Variablengruppen. Abschließend erfolgt nun aufgrund der hohen Anzahl der untersuchten Bedingungen eine tabellarische Zusammenfassung der Ergebnisse. Aufgeführt werden die ausschließlich die signifikanten Variablen. Die Spalte Vorzeichen verdeutlicht, in welcher Beziehung die Variablen zur Nettozinsmarge stehen. Ein positiver Zusammenhang zwischen Variable und Nettozinsmarge wird durch ein Pluszeichen symbolisiert, ein negativer durch ein Minuszeichen. 
Die Spalte Signifikanz verdeutlicht das Signifikanzniveau der getesteten Variablen. Dabei wurde auf die gängige Darstellung des Symbols Stern zurückgegriffen. Die Symbole *, ${ }^{* *},{ }^{* * *}$ repräsentieren Signifikanzniveaus von $10 \%, 5 \%$ und $1 \%$. Sind sie in Klammern gefaßt, so ist die Signifikanz auf dem entsprechenden Niveau nur schwach ausgeprägt. 
Tab. 4-32: Gesamtübersicht der Schätzergebnisse

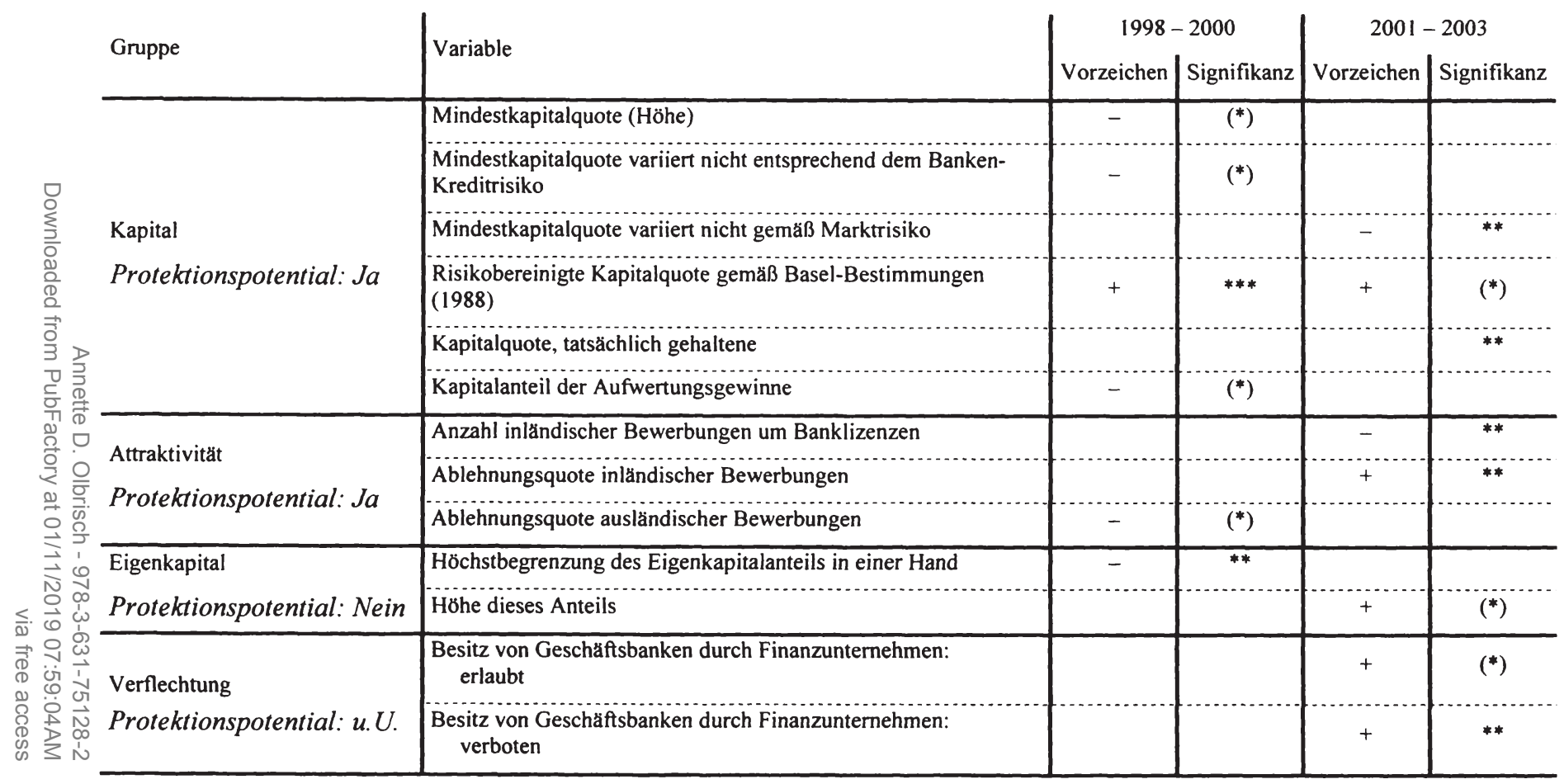


Tab. 4-32: Gesamtübersicht der Schätzergebnisse (Forts.)

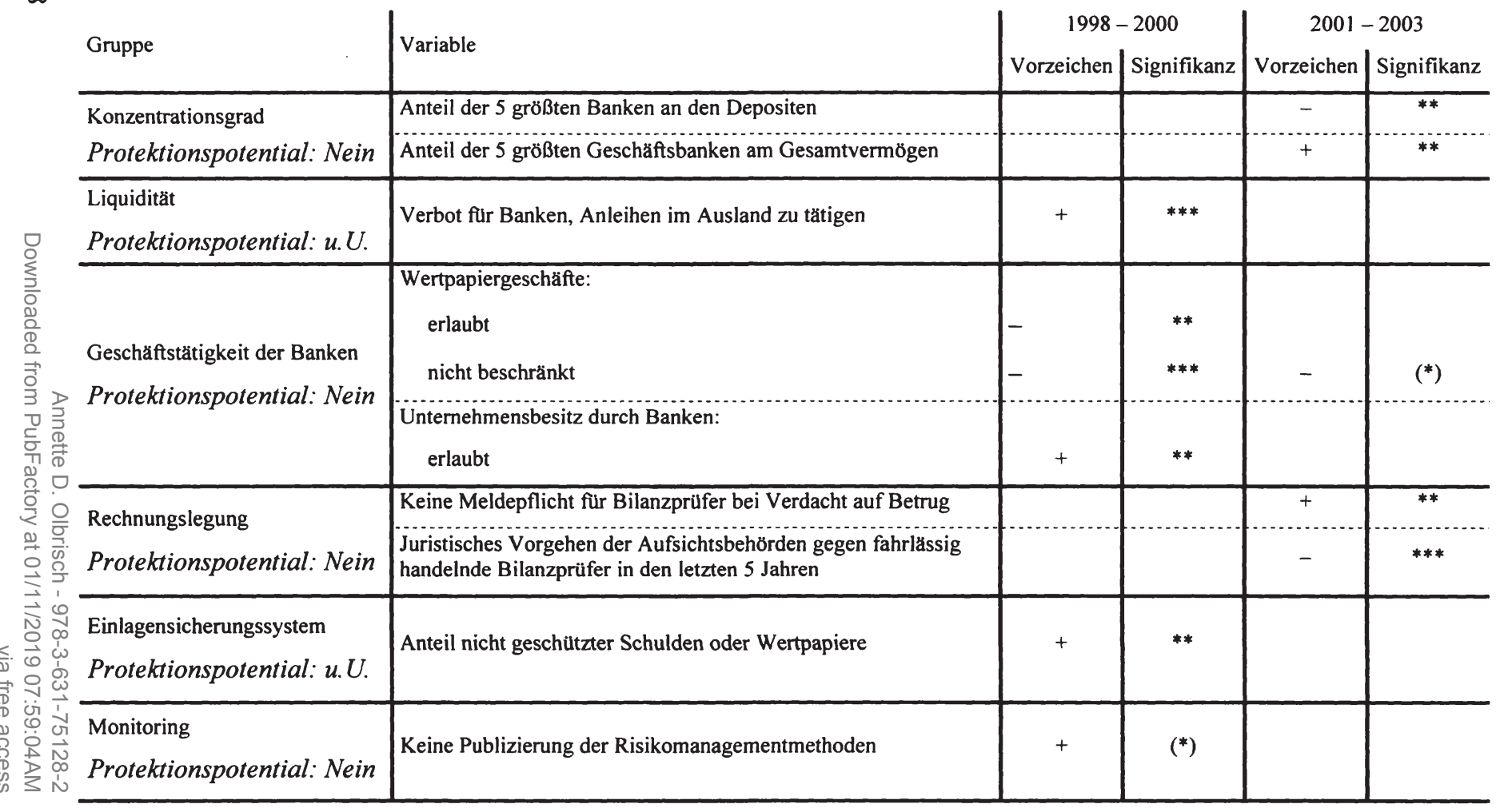


Tab. 4-32: Gesamtübersicht der Schätzergebnisse (Forts.)

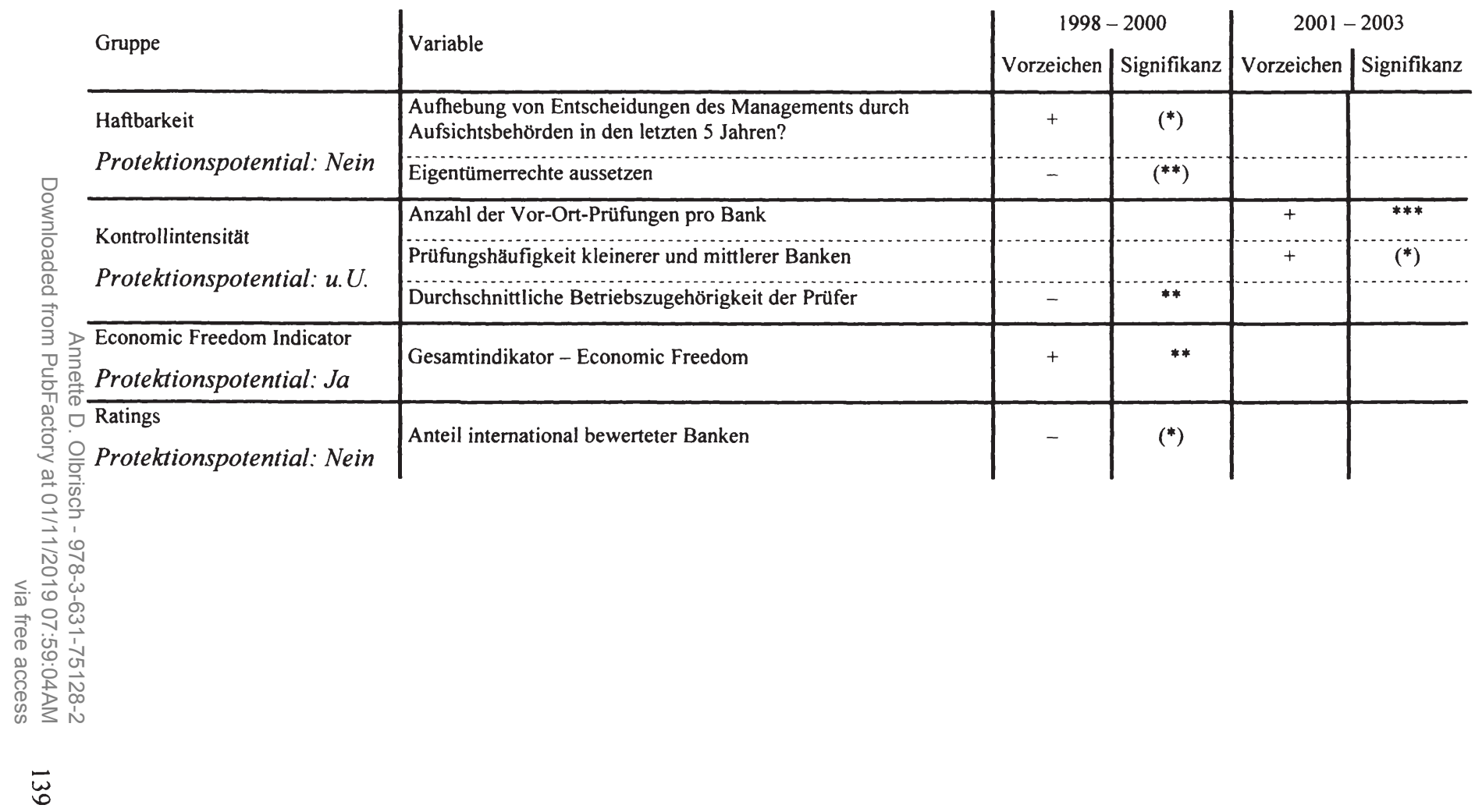


Zur Interpretation der Zahlen wird eine qualitative Bewertung der obigen Ergebnisse vorgenommen. Die reine Betrachtung der Signifikanz einzelner Variablen wird ergänzt um folgendes Punktesystem: Das Symbol *, das einem Signifikanzniveau von $10 \%$ entspricht, wird mit einem Wert von 10 Punkten versehen. Analog werden die Symbole ** sowie ***, die Signifikanzniveaus von $5 \%$ bzw. 1\% repräsentieren, mit den Werten von 20 und 30 Punkten belegt. Die Einfassung der Sternsymbole durch Klammern deutet auf eine schwach ausgeprägte Signifikanz. Entsprechend werden die Symbole $\left({ }^{* *}\right)$ mit 18 und $\left(^{*}\right)$ mit 8 Punkten bewertet.

Tab. 4-33: Bewertung der Signifikanzverteilung

\begin{tabular}{|c|c|c|c|c|}
\hline \multirow{2}{*}{$\begin{array}{l}\text { Bewertung } \\
\text { Signifikanzniveau von } 1 \text { Prozent: }{ }^{* * *}= \\
30 \text { Pkt. }\end{array}$} & \multicolumn{2}{|c|}{$\begin{array}{c}\text { Hăufigkeit in } \\
\text { Periode 1998-2000 } \\
\end{array}$} & \multicolumn{2}{|c|}{$\begin{array}{c}\text { Hăufigkeit in } \\
\text { Periode 2001-2003 }\end{array}$} \\
\hline & 3 & 90 Pkt. & 2 & 60 Pkt. \\
\hline $\begin{array}{l}\text { Signifikanzniveau von } 5 \text { Prozent: } \\
* *=20 \text { Pkt. }\end{array}$ & 6 & 120 Pkt. & 8 & 160 Pkt. \\
\hline $\begin{array}{l}\text { Signifikanzniveau von } 5 \text { Prozent (schwach } \\
\text { ausgeprägt): } \\
\left({ }^{* *}\right)=18 \text { Pkt. }\end{array}$ & 1 & 18 Pkt. & & \\
\hline \multicolumn{5}{|l|}{$\begin{array}{l}\text { Signifikanzniveau von } 10 \text { Prozent: } \\
*=10 \mathrm{Pkt} \text {. }\end{array}$} \\
\hline $\begin{array}{l}\text { Signifikanzniveau von } 1 \text { Prozent (schwach } \\
\text { ausgeprägt): } \\
\left({ }^{*}\right)=8 \text { Pkt. }\end{array}$ & 7 & 56 Pkt. & 5 & 40 Pkt. \\
\hline Summe & & 284 Pkt. & & 260 Pkt. \\
\hline
\end{tabular}

Eine Aufsummierung der Werte liefert uns die Bewertungssummen von 284 Punkten für die erste Periode und von 260 Punkten für die zweite Periode. Insgesamt lässt das die Schlußfolgerung zu, daß die Variablen im ersten Untersuchungszeitraum im Durchschnitt eine höhere Signifikanz aufweisen. Die Zinsmarge wird durch die vorliegenden Variablen im ersten Zeitraum also besser erklärt als im späteren.

\subsubsection{Erkenntnisse aus den Teilschätzungen}

An diese rudimentäre Betrachtung schließt sich nun die Regressionsanalyse aller Variablen auf die Zinsmarge an, wobei für die zwei Untersuchungszeiträume die aussagekräftigsten Schätzgleichungen ermittelt werden. 


\subsubsection{Der erste Untersuchungszeitraum}

Die Regressionsergebnisse belegen, daß die gesetzlich vorgeschriebene Kapitalquote die Höhe der Zinsmargen beeinflußt. Allerdings reagiert die Zinsmarge nicht auf den gesetzlich fixierten Wert, da dieser für alle Banken gleichermaßen gilt, sondern auf die risikobereinigte Kapitalquote, die die individuelle Situation der Banken widerspiegelt. Banken in Ländern, die von einer ökonomisch unsicheren Atmosphäre geprägt sind und in denen folglich das Verlustrisiko ihrer finanziellen Engagements hoch eingeschätzt wird, reagieren mit erhöhter Rücklagenbildung. Dadurch steigen die Kapitalquoten über die erforderliche Mindesthöhe hinaus. Die Signifikanz der Kapitalquote für die Zinsmargenhöhe ist erwiesen. Der Politik stehen als wirtschaftspolitisches Instrument zur Belebung der volkswirtschaftlichen Gesamtsituation alle Bedingungen zur Verfugung, an denen sich die Banken bei der Bestimmung ihrer optimalen Kapitalquote orientieren. Dazu gehören bspw. Aspekte wie die Schaffung eines stabilen ökonomischen Umfeldes, die Reduktion von Unsicherheiten, Gewährung rechtsstaatlicher Rahmenbedingungen. Das Protektionspotential dieser Variable liegt in der diskriminierenden Anwendung von Kapitalquoten. Das zur Verfügung stehende Datenmaterial liegt leider nicht ausreichend detailliert vor, um auszuweisen, ob ausländische Banken zur Haltung höhere Kapitalquoten verpflichtet werden als ihre inländischen Konkurrenten. Hierfür wären eingehendere Befragungen der nationalen Zentralbanken und Aufsichtsbehörden nötig.

In der Variablengruppe „Liquidität" ist das Verbot, Anleihen im Ausland zu tätigen, von hoher Signifikanz für die Nettozinsmarge. Dieses Verbot erhöht die Refinanzierungskosten der Banken, da der Zugang zu internationalen Kapitalmärkten beschränkt wird. Die entsprechend entstehenden Kosten werden durch eine stärkere Spreizung der Zinsen an die Kunden weitergereicht. Durch die Gestaltung dieser Regulierung greift die Wirtschaftspolitik in die Funktionsweise des Bankenmarktes ein. Damit sind die Banken gezwungen, sich allein auf dem inländischen Markt zu refinanzieren. Diese Strategie verhindert einerseits den (Netto-) Abfluß von Devisen, kann sich aber schädlich auf die Finanzierung von Investitionen auswirken, wenn keine ausreichende Kapitalisierung des inländischen Marktes vorliegt. Zumeist haben die inländischen Kreditinstitute aufgrund ihrer oftmals langjährigen Verwobenheit mit dem Inlandsmarkt einen weitaus besseren Zugang zu Depositen und können sich so leichter und in größerem Ausmaß refinanzieren. Die ausländischen Banken sind entsprechend benachteiligt. Diese Regulierung verschafft den inländischen Banken einen erheblichen Wettbewerbsvorteil und dieser Umstand stellt eindeutig Protektion dar.

Je freizügiger den Banken die Betätigung in Geschäftsfeldern wie dem Wertpapierhandel erlaubt wird, um so geringer ist die resultierende Zinsmarge des Landes. Dieser Geschäftsbereich entwickelt sich zu einer bedeutenden Einnahmenquelle der Banken. Ihr Beitrag zum Geschäftsergebnis wirkte sich generell positiv, also senkend, auf die Nettozinsmargen aus. Auch unter dem Aspekt der Risiko- 
diversifizierung ist der Wertpapierhandel der Banken positiv zu bewerten. Durch eine Ausweitung ihrer Tätigkeiten in den das Kreditgeschäft ergänzenden Wertpapierhandel können Banken Risiken besser streuen. Damit reduzieren sie ihre eigene Krisenanfälligkeit und auch die des gesamten Bankensystems. Unter dem Protektionsaspekt ist auf eine diskriminierende Anwendung des Betätigungsverbots hinzuweisen. Wird ausländischen Kreditunternehmen - im Gegensatz zu inländischen Banken - der Wertpapierhandel verboten, so liegt eine eindeutige wettbewerbliche Benachteiligung, also Protektion vor. Die zinsmargenerhöhende Wirkung wird durch die Schätzungen belegt.

Ein zweites zinsmargenrelevantes Geschäftsfeld der Banken stellt der Besitz von inländischen Unternehmen dar. Je mehr Banken mit den inländischen Unternehmen verstrickt sind, um so stärker ist das Wirtschaftssystem gegenüber ausländischen Unternehmen abgeschottet. Ein profitabler Marktzutritt ist ausländischen Banken kaum möglich. Eine derartige Konstellation erinnert an die vielzitierte „Deutschland AG“. Ein hoher Grad an Überkreuz-Beteiligung sicherte das System recht lange gegen kleinere Schocks, es unterband aber auch jegliche Dynamik und wirkte sich negativ auf die Innovationskraft der Unternehmen aus. Während Banken also von einer Erweiterung ihrer originären Geschäftsfelder (Wertpapierhandel) profitieren, wirkt sich die starke Verflechtung mit industriellen Unternehmen ungünstig, d.h. zinsmargenerhöhend, aus. Entsprechend kann die Wirtschaftspolitik über eine Freigabe des Wertpapierhandels und das Begünstigen von Entflechtungen der Besitzverhältnisse senkend auf die Zinsmargen einwirken. Die Schätzergebnisse belegen, daß diese Verflechtungen existieren und staatlicherseits nicht abgebaut werden; damit stellen sie Protektion dar. Der Vergleich der Koeffizienten von -0.018 bzw. -0.035 für die Beschränkung des Wertpapierhandels mit dem Wert von 0.010 belegt, daß die Regulierungen bezüglich des Wertpapierhandels von größerer Wirkung auf die Zinsmargenhöhe sind als Beschränkungen hinsichtlich des Unternehmensbesitzes durch Banken.

In einigen Ländern bleiben bestimmte profitable Geschäftstätigkeiten den heimischen Banken vorbehalten, und ausländischen Konkurrenten wird die Kreditvergabe in den weniger profitablen Wirtschaftsbereichen überlassen (z.B. in Malaysia praktiziert). Durch Diskriminierung ausländischer Unternehmen werden den einheimischen Banken gewinnträchtige Betätigungen gesichert. Diese wirtschaftspolitische Strategie kann im Sinne eines Listschen Erziehungszolls eingesetzt werden. Bei nicht ausreichender Marktkapitalisierung und hohem Kapitalbedarf in den als förderungswürdig eingeschätzten Branchen kann die Regierung durch entsprechende Auflagen ausländischen Banken die Kreditvergabe übertragen. Verglichen mit einer vollständigen Marktabschottung erlaubt dieses Vorgehen die Kapitalisierung bestimmter heimischer (als förderungswürdig betrachteter) Sektoren, ohne ausländischen Banken einen weitreichenden Marktzugang gewähren zu müssen. Allerdings ist der freie Zugang ausländischer Banken zum Kapitalmarkt 
des Landes stets vorzuziehen - dadurch würden alle rentablen Projekte kreditiert, nicht nur die politisch gewollten oder opportunen. ${ }^{1}$

Die Beschränkung des maximalen Eigenkapitalanteils im Besitz einer einzelnen Person bei Banken wirkt sich ebenfalls erhöhend auf die Zinsmargen aus. Damit wird die Investitionstätigkeit natürlicher Personen eingeschränkt, sie können nicht frei über ihr Kapital disponieren. Länder, die derartige Regelungen einführen, verhindern, daß die Kontrolle über eine Bank in der Hand einer einzelnen Person konzentriert wird, aber auch, daß Ausländer die vollständige Kontrolle übernehmen. Als Ergebnis der Regression stellte sich überraschenderweise heraus, daß die Höhe dieser Begrenzung keine Rolle spielt. Die alleinige Existenz dieser Regulierung ist bereits ausreichend, um sich erhöhend auf die Zinsmarge auszuwirken. Diese Bestimmung wird als protektionistisches Instrument eingesetzt. Somit wird staatlicherseits politisch unerwünschte, ausländische Konkurrenz vom Markt ferngehalten bzw. auf ein geringeres Maß beschränkt. Die Protektion, die von dieser Regelung ausgeht ist erheblich, da sie die komplette Marktstruktur zugunsten der inländischen Unternehmen gestaltet.

Anders liegt der Fall beim Einlagensicherungssystem. Hier beeinflußt nicht dessen bloße Existenz die Zinsmargen, sondern nur das Ausmaß des von ihm gewährten Versicherungsschutzes. Je höher der Anteil nicht gedeckter Schulden oder Wertpapiere, um so höher ist das Risiko eines Totalverlustes. Ein unzureichendes Einlagensicherungssystem wirkt sich auch negativ auf die Reputation des inländischen Finanzsystems aus. Der Verdacht, zur Instabilität zu neigen, ist für jede Volkswirtschaft nachteilig. Gerade im Finanzsystem spielt die psychologische Komponente eine gewichtige Rolle. Für eventuelle Verluste, die nicht abgesichert sind, erheben die Banken Risikoaufschlägen, welche wiederum die Zinsmargen erhöhen. Eine protektionistisch agierende Regierung wird - mit Hinweis auf die schlechten juristischen Zugriffsmöglichkeiten auf ausländische Banken deren Prämien höher ansetzen als die inländischer Konkurrenten. Unter diesen Bedingungen engagiert sich eine ausländische Bank auf diesem Markt nur in dem Fall, wenn diesen künstlich erhöhten Betriebskosten auch überdurchschnittlich hohe Gewinne gegenüberstehen. Damit ist eine zusätzliche Marktzugangsbarriere errichtet.

Der Aspekt Berufserfahrung der Kontrolleure (durchschnittliche Betriebszugehörigkeit der Prüfer in den Aufsichtsbehörden) läßt sich ebenfalls wirtschaftspolitisch nutzen. Die Schätzung belegt, daß sich der Einsatz erfahrener Kontrolleure tendenziell zinsmargensenkend auswirkt; die Kontrollen erfolgen effizienter. Die Verwendung von überwiegend unerfahrenen Mitarbeitern in den Aufsichtsbehörden würde zu qualitativ weniger hochwertigen Überprüfungen führen und den Banken einen größeren Gestaltungsspielraum einräumen. Um die Prosperität der

1 Wie bei jeder gezielten Industrie- und Forderungspolitik stellt sich die Frage nach dem Wissensvorsprung der Regierungen hinsichtlich der Auswahl der entwicklungswllrdigen Projekte. 
Volkswirtschaft zu fördern, sollte die Wirtschaftspolitik also Wert darauf legen, daß die Kontrolleure gut ausgebildet werden und Berufserfahrung sammeln. Dieser Aspekt determiniert die Zinsmarge, hat aber keinerlei protektionistische Bedeutung.

Der Economic Freedom Indicator stellte einen bedeutenden Einflußfaktor der Zinsmargen dar. Das Ideal des Indikators stellt eine möglichst freizügige Gestaltung der Wirtschafts- und Sozialbedingungen sowie in außenwirtschaftlicher Dimension die Freihandelsposition dar. Dies beinhaltet die Gestaltung sämtlicher Rahmenbedingungen, die das ökonomische Verhalten der Wirtschaftssubjekte beeinflussen. Dies ist ein weites Feld möglicher Instrumente, das Bereiche wie die Ausgestaltung des Steuersystems, der Sozialversicherungssysteme, administrativer Genehmigungsverfahren, des Rechtssystems u.v.m. umfaßt. Gleichzeitig werden alle Protektionsmaßnahmen erfaßt. Fließt der Economic Freedom Indicator in die Schätzung ein, so erhöht er die Aussagekraft der Regression. Leider kann nicht ermittelt werden, auf welche Faktoren konkret die Beeinflussung zurückzuführen ist. Es handelt sich hierbei um eine catch-all-Größe.

\subsubsection{Der zweite Untersuchungszeitraum}

Fast alle der im ersten Zeitraum zur Gestaltung der Wirtschaftspolitik zur Verfügung stehenden Instrumente verloren im späteren Untersuchungszeitraum an $\mathrm{Be}-$ deutung. Dafür traten andere, potentielle Eingriffsmöglichkeiten in den Vordergrund. Der erweiterte Fragebogen für diesen Zeitraum enthielt Informationen bezüglich einer weiteren Kapitalquote und zwar die nach der aktuell von den Banken gehaltene Höhe der Kapitalquote. Diese weist eine höhere Aussagekraft auf als die risikobereinigte Kapitalquote. Wie schon im ersten Untersuchungszeitraum, so ist auch in der zweiten Untersuchungsperiode nicht die administrativ vorgeschriebene Mindestkapitalquote Determinante der Zinsmargen, sondern die tatsächlich von den Banken gehaltene die entscheidende Größe.

Die Variablengruppe „Attraktivität" untersucht die Anzahl der in- und ausländischen Bewerbungen um Banklizenzen. Sie bietet Ansatzpunkte für steuernde Eingriffe seitens der Wirtschaftspolitik, denn die Bewerbungen inländischer Banken um Lizenzen sind nun von statistischer Signifikanz für die Höhe der Nettozinsmargen. Dieser Umstand repräsentiert die Neustrukturierungen der Bankenmärkte. Hohe Bewerberzahlen sind in Märkten von strategischer Bedeutung oder in Märkten, die sich durch ein hohes Gewinnpotential auszeichnen, zu vermuten. Dieser Umstand belegt, daß nicht der optimale Wettbewerbsgrad erreicht war und durch weitere Markteintritte Renten abgeschöpft bzw. umverteilt wurden. Die neu zugelassenen Banken erhöhen den Wettbewerbsgrad; dies läßt die Zinsmargen tendenziell sinken. Protektionistische Länder unterstützen die Bewerbungen inländischer Banken und unterbinden ausländische Markteintritte. Hierfür bieten sich ein komplettes Zugangsverbot für ausländische Banken an, das immer noch 
viele Länder aufrechterhalten (vgl. GATS-Regularien). Wurde auf ein offizielles Zugangsverbot verzichtet, so bieten sich insbesondere administrative Regelungen an, um den ausländischen Banken die Etablierung zu erschweren. Dazu gehören bspw. aufwendige Genehmigungsverfahren, Beschränkung der Banken hinsichtlich ihrer Aktivitäten in räumlicher oder zeitlicher Dimension etc. Viele Entwicklungsländer verschließen ihre Märkte nach wie vor gegenüber ausländischen Banken, dieser Sachverhalt repräsentiert die direkteste Art der Protektion. Verstärkt die Wirtschaftspolitik hingegen die Deregulierungstendenzen, so wird sie in der Regel mit niedrigeren Zinsmargen belohnt.

Auch das Verbot des Besitzes von Geschäftsbanken durch Finanzunternehmen gewinnt in dem jüngeren Untersuchungszeitraum an Bedeutung. Die Förderung oder Behinderung dieser Art der ökonomischen „Verflechtung “ kann von der Wirtschaftspolitik als protektionistisches Instrument eingesetzt werden. Länder, die diesbezüglich Verbote verhängen, weisen in der Regel höhere Zinsmargen auf. Durch eine starke Verflechtung von Finanz- und Bankbranche profitiert der Finanzstandort als ganzes von einer höheren Effizienz (Synergien); diese Länder weisen niedrigere Zinsmargen auf. Die verstärkte Fusion heimischer Finanzunternehmen kann (bspw. steuerlich) gefördert werden, um Global Player zu erschaffen, die sich auf den internationalen Finanzmärkten durchsetzen können. ${ }^{1}$ Ein hoher Verflechtungsgrad erschwert oder verhindert gar den Marktzutritt ausländischer Banken. Damit wird eine faktische Abschottung des Marktes aufrechterhalten. Unerwünschter ausländischer Konkurrenz wird somit der Marktzugang verwehrt und die inländischen Banken teilen den Markt unter sich auf.

Gleiches gilt für die gestiegene Relevanz des Faktors Konzentrationsgrad. Während dieser im ersten Untersuchungszeitraum keine Rolle spielte, wurden im späteren Untersuchungszeitraum zwei weitere Konzentrationsmaße ermittelt, die sich als Determinanten der Nettozinsmarge erwiesen. Sowohl das Konzentrationsmaß der Depositen wie auch das der Assets ist stark signifikant - allerdings mit unterschiedlichen Vorzeichen. Das auf den Einlagen basierende Konzentrationsmaß geht mit einem negativen Vorzeichen in die Schätzung ein. Eine steigende Einlagenkonzentration geht folglich mit einer sinkenden Zinsmarge einher. Will die Wirtschaftspolitik protektionistisch agieren und den heimischen Banken hohe Gewinnspannen sichern (hohe Zinsmargen), so sollte sie ein stark zergliedertes System der Depositenakkumulation fördern. Hierfür ist der Aufbau weitreichender Filialnetze erforderlich. Ein Markteintritt unter diesen Bedingungen ist für ausländische Banken oftmals zu kostspielig. Wird gleichzeitig auch die Kapitaleinfuhr aus dem Ausland unterbunden, so sind die Chancen auf einen erfolgreichen Markteintritt kaum noch vorhanden. Damit existiert eine wirksame Marktzugangsschranke.

Vgl. Porter (1990), Stichwort „National Champion“. 
Anders liegt der Fall beim asset-basierten Konzentrationsmaß. Es steht in einem positiven Zusammenhang mit der Zinsmarge. Entsprechend läßt eine zunehmende Vermögenskonzentration im Bankensektor die Zinsmargen steigen. Dies läßt wiederum Rückschlüsse auf eine marktbeherrschende Stellung zu. Die Banken sind in der Lage, die Zinsspreizung zu eigenen Gunsten zu vergrößern. Dieser Aspekt spricht für einen Markt mit hohem Angriffspotential: Die bisher in ihm tätigen Banken schöpfen Oligopolgewinne ab. Diese überdurchschnittlich hohen Renditen ziehen auch ausländische Banken an. Protektionismus kann unterstellt werden, wenn die Aufrechterhaltung des derzeitigen Status durch ein Verbot ausländischer Markzutritte forciert wird.

Ein neues Instrumentarium der Wirtschaftspolitik entsteht im Bereich Rechnungslegung/Bilanzierung wie auch Kontrollintensität seitens der Aufsichtsbehörden. Hoch signifikant ist die „Meldepflicht der Bilanzprüfer bei Verdacht auf Betrug“. Eine hohe Zinsmarge repräsentiert also zum Teil qualitativ mangelhafte Kontrollen durch die Bilanzprüfer. Länder, die diese Pflichtmeldung in ihren Bestimmungen vorsehen, zeichnen sich durch eine niedrigere Zinsmarge aus. Die Kontrollen sind effektiver. Ebenso spielt das ,gerichtliche Vorgehen der Aufsichtsbehörden gegen fahrlässige Bilanzprüfer" eine Rolle für die Höhe der Zinsmargen. Durch die Überwachung der Betriebsprüfer sichert die Aufsichtsbehörde die korrekte Bilanzierung der Banken. Argumentativ ähnlich gelagert ist der Aspekt der „Überprüfungshäufigkeit mittlerer und kleinerer Banken“. Häufige, in diesem Fall jährliche, Kontrollen wirken senkend auf die Zinsmargen. Hier kann wiederum die Wirtschaftspolitik ansetzen - ihr obliegt es, den Grad der Kontrolle und dessen Durchsetzung festzulegen.

Im Hinblick auf den Protektionscharakter ist festzustellen, daß der diskriminierende Einsatz der Kontrollen, ausländische Banken erheblich behindert. Die konkrete Ausgestaltung dieser Regulierungen obliegt den Aufsichtsbehörden bzw. den wirtschaftspolitisch Verantwortlichen, welche die Leitlinien vorgeben. Es wird gerade im direkten Vergleich zum vorangegangenen Zeitraum deutlich, daß die Kontrollaktivität und -intensität an Bedeutung für die Determinierung der Zinsmargen hinzugewann. Damit bietet sich der Wirtschaftspolitik ein weites Feld der Betätigung, je nachdem, ob die Zinsmargensenkung Ziel der Wirtschaftspolitik ist oder aber die Marktabschottung gegenüber ausländischen Konkurrenten. Nicht unbeachtet bleiben darf in diesem Zusammenhang der Aspekt, wie die Regulierungen umgesetzt werden. Diskriminierend agierende Beamte können bei ausländischen Banken besonders streng auf die Einhaltung der Regulierungen achten und bei einheimischen Banken etwas großzügiger vorgehen. Damit schaffen sie wertvolle Vorteile für inländische Kreditinstitute.

Im zweiten Untersuchungszeitraum verloren die Bestimmungen zur Einlagensicherung und zur Liquidität (Auslandsanleihen) an Aussagekraft für die Bestimmung der Zinsmargenhöhe. 
Im folgenden Kapitel werden nun alle bisher vorgestellten Variablengruppen gemeinsam einer Regression unterzogen. Das Ziel ist die Identifizierung der aussagekräftigsten Determinanten der Zinsmarge. Aufgrund der Vielzahl von Variablen ist eine langsame Entwickelung der Schlußregression nötig, indem die Variablen nacheinander getestet und nur die vielversprechenden Ansätze weitergeführt werden. Diese Vorgehensweise erfordert auch den erneuten Test einmal bereits verworfener Variablen. Es handelt sich hierbei um ein sehr aufwendiges Verfahren, da sich die Variablen z.T. untereinander beeinflussen und in den Wirkungen überdecken. Die Variablen mit dem größten Erklärungsgehalt herauszufiltern, ist nur so möglich. Interessanterweise gewinnt eine Variablen in der Gesamtschätzung des zweiten Untersuchungszeitraumes an Signifikanz, die sie bei den Einzelregressionen der Variablengruppen nicht besaß.

\subsection{Die aussagekräftigsten Schätzgleichungen}

Die zunächst vollzogenen Teilschätzungen analysierten detailliert, welche Determinanten für die Bestimmung der Höhe der Zinsmargen wichtig sind. Somit ist es möglich, die Veränderung der Bedeutung der geprüften Variablen im Zeitablauf für die Zinsmargenhöhe zu verdeutlichen. Es werden nun die besten Schätzgleichungen unter Verwendung aller Variablen für die beiden Zeiträume ermittelt. Dabei werden die Variablen herausgestellt, die die Unterschiede in den Zinsmargen am besten erklären.

\subsubsection{Aussagekräftigste Schätzgleichung 1998-2000}

Für den Zeitraum von 1998 bis 2000 wurde als aussagekräftigste Schätzgleichung der Nettozinsmarge ermittelt:

$$
\begin{aligned}
N I M_{,}= & \alpha+\beta_{1}^{*} G D P p c_{i}+\beta_{2}^{*} d 34_{i}+\beta_{3}^{*} d 21_{i}+\beta_{4}^{*} d 411_{i}+\beta_{5}^{*} d 412_{i}+\beta_{6}^{*} d 116_{i}+ \\
& \beta_{7}^{*} E F I_{i}^{F B}+\beta_{8}^{*} E F I_{i}^{M P}+\beta_{9}^{*} E F I_{i}^{F I}+\beta_{10}^{*} E F I_{i}^{W P}+u_{i}
\end{aligned}
$$


mit:

$\begin{array}{ll}\alpha & =\text { Konstanter Parameter, } \\ \beta_{1}, \ldots, \beta_{10} & =\text { Konstante, geschätzte Koeffizienten, } \\ N I M_{i} & =\text { Zinsmarge des Landes } i \\ G D P p c_{i} & =\text { Pro-Kopf-Einkommen des Landes } i \\ d 34_{i} & =\text { Kapitalquote des Landes } i \\ d 21_{i} & =\text { Eigenkapitalbeschränkung, } \\ d 411_{i} & =\text { Nicht beschränkter Wertpapierhandel, } \\ d 412_{i} & =\text { Beschränkter Wertpapierhandel, } \\ d 116_{i} & =\text { Aufhebung von Management-Beschlüssen, } \\ E F I_{i}^{F B} & =\text { Economic Freedom Indicator: Fiscal Burden, } \\ E F I_{i}^{M P} & =\text { Economic Freedom Indicator: Monetary Policy, } \\ E F I_{i}^{F I} & =\text { Economic Freedom Indicator: Foreign Investment, } \\ E F I_{i}^{W P} & =\text { Economic Freedom Indicator: Wages and Prices, } \\ u_{i} & =\text { Störgröße des Landes } i .\end{array}$


Abb. 4-1: Aussagekräftigste Schätzgleichung 1998 - 2000

\begin{tabular}{lccc} 
Source & SS & df & MS \\
\hline Model & 0.030145805 & 10 & 0.003014581 \\
Residual & 0.007850248 & 46 & 0.000170658 \\
\hline Total & 0.037996054 & 56 & 0.000678501
\end{tabular}

$\begin{array}{rlr}\text { Num ber of obs. } & = & 57 \\ \mathrm{~F}(10,46) & = & 17.66 \\ \text { Prob }>\mathrm{F} & = & 0.0000 \\ \mathrm{R}^{2} & = & 0.7934 \\ \mathrm{Adj} . \mathrm{R}^{2} & = & 0.7485 \\ \text { Root MSE } & = & 0.01306\end{array}$

\begin{tabular}{r|rrrrrr}
\hline d95 & \multicolumn{1}{l}{ Coef. } & Std. Err. & \multicolumn{1}{l}{ t } & \multicolumn{1}{l}{ Pr. } & \multicolumn{1}{l}{ [95\% Conf. Interval] } \\
\hline GDPpc & -0.0009502 & 0.0002585 & -3.68 & 0.001 & -0.0014705 & -0.00043 \\
$\mathrm{~d} 34$ & 0.081811 & 0.0416488 & 1.96 & 0.056 & -0.0020238 & 0.1656457 \\
$\mathrm{~d} 21$ & -0.0147085 & 0.0040088 & -3.67 & 0.001 & -0.0227777 & -0.0066392 \\
$\mathrm{~d} 411$ & -0.0133056 & 0.0066051 & -2.01 & 0.050 & -0.026601 & -0.0000102 \\
$\mathrm{~d} 412$ & -0.0190544 & 0.0069612 & -2.74 & 0.009 & -0.0330665 & -0.0050423 \\
$\mathrm{~d} 116$ & 0.0104127 & 0.0038576 & 2.70 & 0.010 & 0.0026477 & 0.0181776 \\
EFI - Fiscal Burden & 0.0057415 & 0.0026987 & 2.13 & 0.039 & 0.0003092 & 0.0111737 \\
EFI - M onetary Policy & 0.0069301 & 0.0020529 & 3.38 & 0.002 & 0.0027978 & 0.0110624 \\
EFI - Foreign Invest. & 0.0084274 & 0.0031943 & 2.64 & 0.011 & 0.0019976 & 0.0148571 \\
EFI - Wages \& Prices & -0.0084334 & 0.00321 & -2.63 & 0.012 & -0.0148948 & -0.001972 \\
Cons. & 0.0238689 & 0.017094 & 1.40 & 0.169 & -0.0105395 & 0.0582773 \\
\hline
\end{tabular}

Darstellung des STATA-Ergebnistableaus. 
Regression with robust standard errors

$$
\begin{array}{rlr}
\text { Number of obs. } & & 57 \\
\text { F }(10,46) & =25.45 \\
\text { Prob }>\text { F } & =0.0000 \\
\mathrm{R}^{2} & =0.7934 \\
\text { Root MSE } & =0.01306
\end{array}
$$

\begin{tabular}{|c|c|c|c|c|c|c|}
\hline d95 & Coef. & $\begin{array}{l}\text { Robust } \\
\text { Std. Err. }\end{array}$ & $\mathbf{t}$ & Pr. & \multicolumn{2}{|c|}{ [ $95 \%$ Conf. Interval] } \\
\hline GDPpc & -0.0009502 & 0.0002449 & -3.88 & 0.000 & -0.0014433 & -0.0004572 \\
\hline d34 & 0.081811 & 0.0285479 & 2.87 & 0.006 & 0.024347 & 0.1392749 \\
\hline d21 & -0.0147085 & 0.0041266 & -3.56 & 0.001 & -0.0230148 & -0.0064021 \\
\hline d411 & -0.0133056 & 0.0077652 & -1.71 & 0.093 & -0.0289342 & 0.0023229 \\
\hline d412 & -0.0190544 & 0.0076118 & -2.50 & 0.016 & -0.0343762 & -0.0037326 \\
\hline d 116 & 0.0104127 & 0.0036252 & 2.87 & 0.006 & 0.0031156 & 0.0177097 \\
\hline EFI - Fiscal Burden & 0.0057415 & 0.0025411 & 2.26 & 0.029 & 0.0006265 & 0.0108564 \\
\hline EFI - Monetary Policy & 0.0069301 & 0.0019431 & 3.57 & 0.001 & 0.0030189 & 0.0108413 \\
\hline EFI - Foreign Invest. & 0.0084274 & 0.0033439 & 2.52 & 0.015 & 0.0016964 & 0.0151584 \\
\hline EFI - Wages \& Prices & -0.0084334 & 0.0028009 & -3.01 & 0.004 & -0.0140713 & -0.0027955 \\
\hline Cons. & 0.0238689 & 0.0151857 & 1.57 & 0.123 & -0.0066983 & 0.0544362 \\
\hline
\end{tabular}

Darstellung des STATA-Ergebnistableaus. 
Den höchsten Erklärungswert in diesem Zeitraum für die Zinsmargenhöhe weisen folgende Variablen auf:

- Pro-Kopf-Einkommen (GDPpc),

- Kapitalquote (d34),

- Beschränkung des Eigenkapitals einer Bank in der Hand einer Person (d21),

- Nicht beschränkte Betätigung der Banken im Wertpapierhandel (d411),

- Erlaubte Betätigung der Banken im Wertpapierhandel (mit minimalen Beschränkungen) (d412),

- Aufhebung von Beschlüssen des Bankenmanagements hinsichtlich Dividendenausschüttung, Bonuszahlung oder Gebührenhöhe durch die Aufsichtsbehörden (d116) sowie

- die Teilindikatoren der ökonomischen Freiheit: Fiskalische Belastung ${ }^{1}$ durch den Staat, Geldpolitik, Auslandsinvestitionen und die Höhe der Löhne und Preise.

Die Schätzung weist ein Bestimmtheitsmaß $\left(\mathrm{R}^{2}\right)$ von 0,7934 und ein adjustiertes Bestimmtheitsma $\beta$ von 0,7485 auf. Die ermittelten Variablen erklären also annähernd 75 Prozent der Varianz in den Zinsmargen. Die Anzahl der Beobachtungen liegt bei 56, weil die Daten nicht vollständig für alle Länder vorliegen. Allerdings zeichnet sich die Schätzung durch 46 Freiheitsgrade aus. Die ermittelten Variablen weisen hohe Signifikanzwerte auf: Sechs Variablen sind zum 1-Prozent-Niveau signifikant, drei Variablen zum 3-Prozent-Niveau und eine zum 10Prozent-Niveau.

Führt man eine robuste Schätzung durch, um für Heteroskedastizität zu korrigieren (robust standard errors), so werden die Ergebnisse grundsätzlich bestätigt. Allerdings verliert die Variable „nicht beschränkte Betätigung der Banken im Wertpapierhandel" (d411) etwas an Signifikanz (nur noch schwach signifikant zum 10-Prozent-Niveau).

Die eingehende Betrachtung der einzelnen Determinanten führt $\mathrm{zu}$ folgender Interpretation:

- Die Kapitalquote (d34) geht mit einem positiven Vorzeichen in die Regression ein. Das bedeutet, daß die Existenz einer gesetzlich vorgeschriebenen Kapitalquote die Zinsmargen beeinflußt: Je höher die Kapitalquote in einem Land festgesetzt wird, um so höher sind die Zinsmargen

Hierunter werden die Einkommens- und Unternehmenssteuern aber auch der offentliche Verschuldungsstand subsumiert. 
des Landes. Folglich setzen die wirtschaftspolitischen Akteure durch Festsetzung der Kapitalquote das Niveau der Zinsen fest: Hohe Kapitalquoten bedeuten für die Banken die Bindung von relativ viel Kapital, das nicht im Kreditgeschäft produktiv verwendet werden darf. Somit stellen sie einen erhöhten Kostenfaktor dar. Entsprechend heben die Banken ihre Kreditzinsen an. Unter dem Aspekt der Protektion ist diese Variable differenziert zu betrachten. Ist von in- und ausländischen Banken dieselbe Kapitalquote zu erbringen, so liegt keine Benachteiligung ausländischer Banken und damit auch keine Protektion vor. Nur wenn unterschiedliche, d.h. höhere Kapitalquoten für ausländische als für inländische Banken gelten, wäre Protektion belegt. Hierfür liefert die vorliegende Datenbasis jedoch keinen Hinweis, da sie nicht ausreichend differenziert genug erhoben wurde. Somit ist im Ergebnis die Kapitalquote eine Determinante der Zinsmarge, welche Protektionspotential birgt. Tatsächliche Protektion kann aber aufgrund der vorliegenden Datenbasis nicht nachgewiesen werden, solange Beweise für die Ungleichbehandlung von in- und ausländischen Unternehmen nicht vorliegen.

- Nicht beschränkte Betätigung der Banken im Wertpapierhandel (d411) sowie die erlaubte Betätigung der Banken im Wertpapierhandel (mit minimalen Beschränkungen) (d412): Beide Variablen gehen mit einem negativen Vorzeichen in die Regression ein. Die ökonomische Schlußfolgerung lautet entsprechend, daß je größer das Ausmaß an erlaubtem Wertpapierhandel ist, um so niedriger ist die Zinsmarge eines Landes. Diese Variable ist also unter dem Aspekt der Geschäfts- und Risikodiversifizierung von großer Bedeutung. Ein Betätigungsverbot ausländischer Banken in diesem Geschäftsfeld ist eindeutig protektionistisch, denn dadurch werden inländische Kreditinstitute bevorteilt. Hier bietet sich der Wirtschaftspolitik ein protektionistisches Instrument zum Schutz der heimischen Banken.

- Aufhebung von Beschlüssen des Bankenmanagements hinsichtlich Dividendenausschüttung, Bonuszahlung oder Gebührenhöhe durch die Aufsichtsbehörden (d116): Diese Variable zur Konkretisierung der Zugriffsrechte der Aufsichtsbehörden geht mit einem positiven Vorzeichen in die Regressionsgleichung ein: Je weniger die Behörden von diesem Recht Gebrauch machen, bzw. je weniger ausgeprägt diese Zugriffsrechte für die Behörden sind, um so höher die Zinsmarge des Landes. Dieser Umstand erklärt sich daraus, daß ein Mangel an Durchsetzungsstärke der Autoritäten es den Banken erlaubt, ihre Marktposition zur Erhöhung der Kreditzinsen bzw. Senkung der Depositenzinsen zu nutzen. Auf den Umstand, daß Bürokraten (auch durchaus unbewußt) dazu neigen, inländische Unternehmen zu schützen und ausländische Konkurrenten eher strenger zu kontrollieren, wurde bereits an anderer Stelle hingewiesen. 
- Beschränkung des Eigenkapitals einer Bank in der Hand einer Person (d21): Die Variable geht negativ in die Schätzgleichung ein. Dies bedeutet, daß die Existenz einer Kapitalbeschränkung sich erhöhend auf die Zinsmarge auswirkt. Diese Regulierung wirkt abschreckend auf die investitionswilligen ausländischen Banken, da sie genötigt sind, inländische Kooperationspartner zu beteiligen.

- Die Teilindikatoren zur Messung ökonomischer Freiheit messen das Ausmaß der vorherrschenden Regulierungen und Protektionsmaßnahmen in verschiedenen Bereichen. Die Steuern- und Abgabenbelastung des privaten Sektors, die geldpolitische Maßnahmen wie auch die Höhe der Auslandsinvestitionen stehen in einem positiven Zusammenhang mit der Zinsmarge. Je weniger regulativ diese Bereiche ausgeprägt sind, d.h. je höher der Grad ökonomischer Freiheit ist, um so niedriger ist die Zinsmarge des Landes.

Verwendet man als Erklärungsvariable den Gesamtindikator für ökonomische Freiheit anstelle der vier Teilindikatoren, so lauten die Ergebnisse: 


\begin{tabular}{lcrr} 
Source & SS & df & \multicolumn{1}{c}{ MS } \\
\hline Model & 0.027323711 & 8 & 0.003415464 \\
Residual & 0.010672343 & 48 & 0.00022234 \\
\hline Total & 0.037996054 & 56 & 0.000678501
\end{tabular}

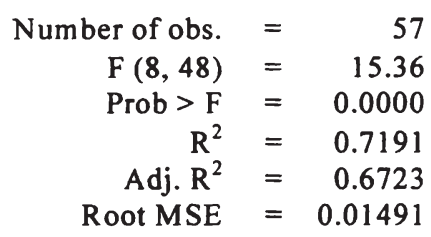

\begin{tabular}{r|rrrrrr}
\hline $\mathrm{d} 95$ & \multicolumn{1}{l}{ Coef. } & Std. Err. & $\mathrm{t}$ & \multicolumn{1}{l}{ Pr. } & \multicolumn{2}{l}{ [95\% Conf. Interval] } \\
\hline GDPpc & -0.0008249 & 0.0002833 & -2.91 & 0.005 & -0.0013945 & -0.0002554 \\
$\mathrm{~d} 34$ & 0.1130326 & 0.0408425 & 2.77 & 0.008 & 0.0309132 & 0.1951519 \\
$\mathrm{~d} 21$ & -0.0150143 & 0.0044095 & -3.40 & 0.001 & -0.0238801 & -0.0061484 \\
$\mathrm{~d} 411$ & -0.0159422 & 0.0071353 & -2.23 & 0.030 & -0.0302886 & -0.0015957 \\
$\mathrm{~d} 412$ & -0.0287195 & 0.0071091 & -4.04 & 0.000 & -0.0430132 & $-0,0144258$ \\
$\mathrm{~d} 443$ & -0.0085467 & 0.004249 & -2.01 & 0.050 & -0.01709 & -0.0000035 \\
$\mathrm{~d} 116$ & 0.0140605 & 0.0040863 & 3.44 & 0.001 & 0.0058445 & 0.0222765 \\
Economic Freedom & 0.0115058 & 0.0053134 & 2.17 & 0.035 & 0.0008224 & 0.0221891 \\
Indicator & 0.0329486 & 0.0199977 & 1.65 & 0.106 & -0.0072595 & 0.0731568 \\
Cons. & & & & & &
\end{tabular}


Abb. 4-4: Aussagekräftigste Schätzgleichung 1998 - 2000, Economic Freedom Gesamtindikator (robuste Schätzung)

Regression with robust standard errors

$$
\begin{array}{rlr}
\text { Number of obs. } & =57 \\
\mathrm{~F}(8,48) & =18.71 \\
\text { Prob }>\mathrm{F} & =0.0000 \\
\mathrm{R}^{2} & =0.7191 \\
\text { Root MSE } & =0.01491
\end{array}
$$

\begin{tabular}{r|lllllr}
\hline $\mathrm{d} 95$ & Coef. & $\begin{array}{c}\text { Robust } \\
\text { Std. Err. }\end{array}$ & $\mathrm{t}$ & Pr. & \multicolumn{2}{c}{ [95\% Conf. Interval] } \\
\hline $\mathrm{GDPpc}$ & -0.0008249 & 0.0002479 & -3.33 & 0.002 & -0.0013234 & -0.0003265 \\
$\mathrm{~d} 34$ & 0.1130326 & 0.0288652 & 3.92 & 0.000 & 0.0549951 & 0.17107 \\
$\mathrm{~d} 1$ & -0.0150143 & 0.0043424 & -3.46 & 0.001 & -0.0237453 & -0.0062832 \\
$\mathrm{~d} 411$ & -0.0159422 & 0.0074235 & -2.15 & 0.037 & -0.0308682 & -0.0010161 \\
$\mathrm{~d} 412$ & -0.0287195 & 0.0071551 & -4.01 & 0.000 & -0.0431059 & -0.0143331 \\
$\mathrm{~d} 443$ & -0.0085467 & 0.0045913 & -1.86 & 0.069 & -0.0177781 & 0.0006846 \\
$\mathrm{~d} 116$ & 0.0140605 & 0.0039578 & 3.55 & 0.001 & 0.0061029 & 0.0220181 \\
Economic Freedom & 0.0115058 & 0.0048914 & 2.35 & 0.023 & 0.0016709 & 0.0213406 \\
Indicator & 0.0329486 & 0.0187106 & 1.76 & 0.085 & -0.0046716 & 0.0705689 \\
Cons. & 0 & & & & &
\end{tabular}

Darstellung des STATA-Ergebnistableaus. 
Auch in dieser Schätzung sind die gleichen Variablen relevant:

- Pro-Kopf-Einkommen, Kapitalquote, Beschränkung des Eigenkapitals einer Bank in der Hand einer Person, nicht beschränkter Wertpapierhandel, Wertpapierhandel mit minimalen Beschränkungen, erfolgte Aufhebung von Beschlüssen des Bankenmanagements hinsichtlich Dividendenausschüttung, Bonuszahlung oder Gebührenhöhe durch die Aufsichtsbehörden sowie der Gesamtindikator für ökonomische Freiheit.

Neu hinzugetreten ist die Variable:

- Beschränkung des Besitzes von Nicht-Finanzunternehmen durch Banken (d443).

Offensichtlich wurde der Einfluß dieser Variablen durch die Teilindikatoren zur ökonomischen Freiheit überdeckt. Insgesamt weisen fünf Variablen eine hohe Signifikanz zum 1-Prozent-Niveau auf, drei Variablen sind signifikant zum 5-Prozent-Niveau. Das Bestimmtheitsmaß $\mathrm{R}^{2}$ liegt bei 0,7191 und das adjustierte $\mathrm{Be}-$ stimmtheitsmaß bei 0,6723 . Die robuste Schätzung bestätigt die Signifikanz der Variablen.

\subsubsection{Aussagekräftigste Schätzgleichung 2001 - 2003}

Für den Zeitraum von 2001 bis 2003 wurde als Schätzgleichung mit dem höchsten Aussagegehalt ermittelt:

$$
\begin{aligned}
N I M_{i}= & \alpha+\beta_{1}^{*} G D P p c_{i}+\beta_{2} * d 342_{i}+\beta_{3}^{*} d 56_{i}+\beta_{4} * d 125_{1}+\beta_{5}^{*} d 58_{1}+\beta_{6} * d 253_{1}+ \\
& \beta_{7}^{*} d 233_{i}+\beta_{8} * d 111_{i}+\beta_{9}^{*} d 412_{i}+u_{i}
\end{aligned}
$$

mit:

$$
\begin{array}{ll}
\alpha & =\text { Konstanter Parameter, } \\
\beta_{1}, \ldots, \beta_{9} & =\text { Konstante, geschätzte Koeffizienten, } \\
N I M_{i} & =\text { Zinsmarge des Landes } i \\
G D P p c_{i} & =\text { Pro-Kopf-Einkommen des Landes } i \\
d 342_{i} & =\text { Kapitalquote der Banken des Landes } i \\
d 56_{i} & =\text { Pflichtmeldung bei Verdacht auf eine Straftat, }
\end{array}
$$




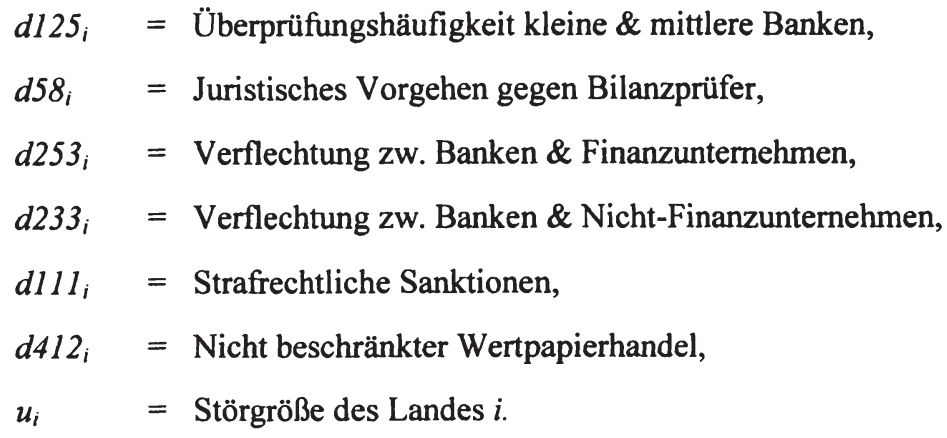

In diesem Zeitraum sind die bestimmenden Regressoren:

- Pro-Kopf-Einkommen (GDPpc),

- Tatsächliche Kapitalquote der Banken (d342),

- Pflichtmeldung der Bilanzprüfer bei der Aufsichtsbehörde bei Verdacht auf eine Straftat oder Hinterziehung (d56),

- Überprüfungshäufigkeit mittlerer und kleinerer Banken (d125),

- Juristisches Vorgehen der Aufsichtsbehörden gegen fahrlässige Bilanzprüfer in den letzten 5 Jahren (d58),

- Beschränkter Besitz von Geschäftsbanken durch Finanzunternehmen (d253),

- Beschränkter Besitz von Geschäftsbanken durch Nicht-Finanzunternehmen (d233),

- Existenz von Mechanismen in Form von Unterlassungsanordnungen, die automatisch zu zivil- oder strafrechtlichen Sanktionen bei Fehlverhalten der Bankmanager führen (d111),

- nicht beschränkter Wertpapierhandel (d412).

Die Schätzung weist ein Bestimmtheitsmaß von 0,5908 und ein adjustiertes Bestimmtheitsmaß von 0,5262 auf. Dies bedeutet, daß mit diesen Variablen annähernd 53 Prozent der Varianz in den Zinsmargen erklärt werden können.

Die Variablen weisen in diesem Zeitraum keine so hohe Signifikanz mehr auf. Von hoher Signifikanz zum 1-Prozent-Niveau sind die Variablen GDPpc, d342, d58, d111 und d412. Signifikant zum 3-Prozent-Niveau ist die Variable d56. Nur schwache Signifikanz zum 10-Prozent-Niveau weisen die Variablen d253 und d233 auf. Einen Sonderfall stellt die Variable d125 „Überprüfungshäufigkeit kleiner und mittlerer Banken" dar: Sie ist in der Ursprungsschätzung nicht signifi- 
kant (Ablehnungswahrscheinlichkeit von 15,8 Prozent). Im Test auf Heteroskedastizität wird sie dann aber zu einer schwach signifikanten Variablen mit einer Ablehnungswahrscheinlichkeit von nur noch 5,7 Prozent.

Auch in der zweiten Beobachtungsperiode bestätigt die robuste Schätzung (Heteroskedastizität) grundsätzlich die Ergebnisse. Nur die Variable „,beschränkter Besitz von Geschäftsbanken durch Nicht-Finanzunternehmen“ (d233) verliert ihre Signifikanz.

Unter dem Aspekt der Protektion sind analog zu den Ausführungen in Kapitel 4.1.3 Variablengruppen und ihre Protektionspotentiale folgende Variablen interessant:

- Überprüfungshäufigkeit mittlerer und kleinerer Banken (d125): Diese Variable geht positiv in die Regression ein. Das bedeutet, daß diejenigen Länder, die eine hohe Prüffrequenz aufweisen, auch über die niedrigeren Zinsmargen verfügen. Wie intensiv wiederum die regelmäßigen Vor-OrtKontrollen der Banken durchgeführt werden, hängt von der Einstellung der durchführenden Beamten $a b$ - es ist vernünftig zu vermuten, daß ausländische Banken strengeren Kontrollen ausgesetzt sind. Damit stellen die Kontrollen ein Instrument dar, das in diskriminierender Weise ausländische Banken benachteiligt.

- Pflichtmeldung der Bilanzprüfer bei der Aufsichtsbehörde bei Verdacht auf eine Straftat oder Hinterziehung (d56): Diese Variable ist in der Schätzung mit einem positiven Vorzeichen versehen, d.h., daß diejenigen Länder, die Pflichtmeldungen bei entdeckten Verstößen vorsehen, niedrigere Zinsmargen aufweisen. Diese Regelung zwingt die Bilanzprüfer in ein neutrales Verhältnis gegenüber ihren Auftraggebern, den Banken. Damit wird die Abfassung unabhängiger und korrekter Testate gesichert und verhindert, daß Gefälligkeitsgutachten entstehen. Die realistische Bilanzierung der Banken senkt das Betrugsrisiko und deckt mögliche Risiken auf. Dies reduziert die Unsicherheiten und im Ergebnis wirkt sich die Regulierung senkend auf die Zinsmargen aus. Diese Variable determiniert die Zinsmarge, läßt sich aber nicht in einem protektionistischen Sinne verwenden. Mit dieser Variable inhaltlich eng verbunden ist das

- Juristische Vorgehen der Aufsichtsbehörden gegen fahrlässige Bilanzprüfer in den letzten 5 Jahren (d58): Die Variable geht negativ in die Schätzgleichung ein. Je weniger Vorfälle es gab, um so niedriger war die Zinsmarge. D.h. eine niedrige Zahl von Vorfällen belegt die qualitativ hochwertigen Kontrollen der bilanzprüfenden Unternehmen in einem Land. Auch diese Variable läßt sich nicht für protektionistische Zwecke instrumentalisieren. 
Abb. 4-5: Aussagekräftigste Schätzgleichung 2001 - 2003

\begin{tabular}{lrrc} 
Source & \multicolumn{1}{c}{ SS } & df & MS \\
\hline Model & 0.03072253 & 9 & 0.003413614 \\
Residual & 0.021276429 & 57 & 0.000373271 \\
\hline Total & 0.051998959 & 66 & 0.000787863
\end{tabular}

$$
\begin{array}{rlr}
\text { Num ber of obs. } & = & 67 \\
\mathrm{~F}(9,57) & =9.15 \\
\text { Prob }>\mathrm{F} & =0.0000 \\
\mathrm{R}^{2} & =0.5908 \\
\text { Adj. } \mathrm{R}^{2} & =0.5262 \\
\text { Root MSE } & =0.01932
\end{array}
$$

\begin{tabular}{r|rrrrrr}
\hline$d 95$ & \multicolumn{1}{l}{ Coef. } & \multicolumn{1}{l}{ Std. Err. } & \multicolumn{1}{l}{ t } & \multicolumn{1}{l}{ Pr. } & \multicolumn{1}{l}{ [95\% Conf. Interval] } \\
\hline GDPpc & -0.000748 & 0.0002571 & -2.91 & 0.005 & -0.0012627 & -0.0002332 \\
$\mathrm{~d} 342$ & 0.1795878 & 0.0390502 & 4.60 & 0.000 & 0.101391 & 0.2577845 \\
$\mathrm{~d} 56$ & 0.0148863 & 0.0057106 & 2.61 & 0.012 & 0.003451 & 0.0263216 \\
$\mathrm{~d} 125$ & 0.0078269 & 0.0054678 & 1.43 & 0.158 & -0.0031222 & 0.018776 \\
$\mathrm{~d} 58$ & -0.163044 & 0.0061512 & -2.65 & 0.010 & -0.0286219 & -0.0039868 \\
$\mathrm{~d} 253$ & -0.0180719 & 0.0095724 & -1.89 & 0.064 & -0.0372404 & 0.0010966 \\
$\mathrm{~d} 233$ & 0.0141673 & 0.0076947 & 1.84 & 0.071 & -0.001241 & 0.0295756 \\
$\mathrm{~d} 111$ & 0.014894 & 0.0055 & 2.71 & 0.009 & 0.0038804 & 0.0259076 \\
d412 & -0.0151056 & 0.0055711 & -2.71 & 0.009 & -0.0262615 & -0.0039497 \\
Cons. & 0.0293637 & 0.0081152 & 3.62 & 0.001 & 0.0131132 & 0.0456142 \\
\hline
\end{tabular}

Darstellung des STATA-Ergebnistableaus. 


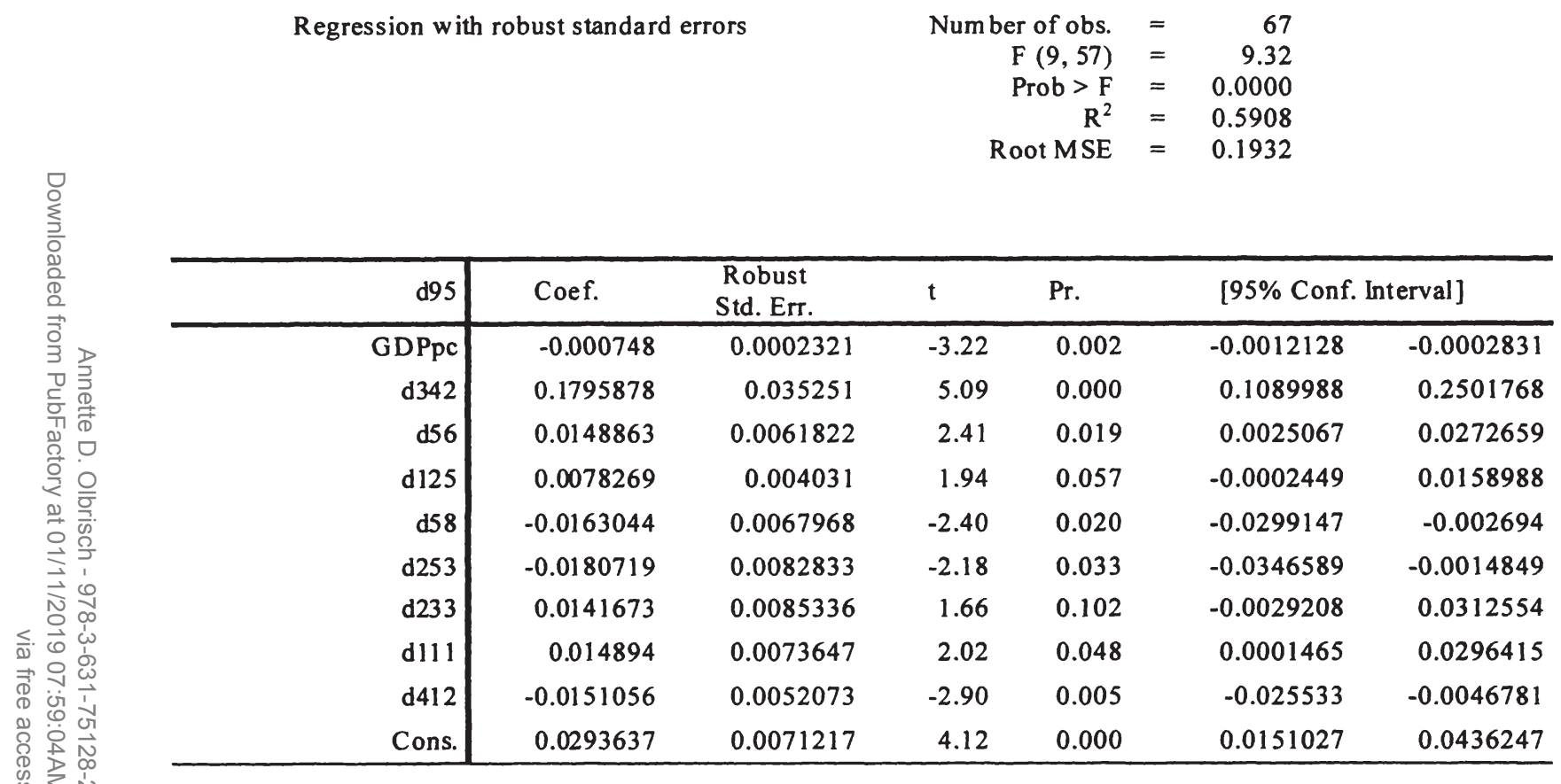


- Mechanismen, die automatisch zu Sanktionen bei Fehlverhalten der Bankmanager führen (d111): Diese Variable geht mit einem positiven Vorzeichen in die Regression ein. Länder, die über derartige Bestrafungsmechanismen verfügen, weisen relativ niedrige Zinsmargen auf. Demgegenüber herrschen in Ländern, die eher über laxe Vorschriften hinsichtlich der Verfolgung von straffälligen Bankmanagern verfügen, hohe Zinsmargen vor. Protektionistisch wäre diese Regulierung nur bei einer abhängigen und weisungsgebundenen Justiz nutzbar - ein Umstand, der sich nur noch in Diktaturen wiederfindet.

- Beschränkter Besitz von Geschäftsbanken durch Finanzunternehmen (d253): Diese Variable geht mit einem negativen Vorzeichen in die Schätzung ein. Damit lautet die Wirkungskette, daß eine Behinderung der Verflechtung zu steigenden Zinsmargen führt. Je stärker verschiedene Unternehmen der Finanzbranche miteinander verwoben sind, um so effizienter erfolgt die Versorgung des Landes mit Bankdienstleistungen. Dieser $\mathrm{Zu}-$ sammenhang läßt sich an entsprechend niedrigen Zinsmargen ablesen. Somit bestätigt auch die Gesamtschätzung die Gültigkeit der Efficient Structure Theory für den Finanzsektor.

- Beschränkter Besitz von Geschäftsbanken durch Nicht-Finanzunternehmen (d233): Diese Variable hingegen geht mit einem positiven Vorzeichen in die Regressionsgleichung ein. Ein steigender Grad der Verflechtung zwischen Banken und Unternehmen außerhalb des Finanzsektors wirkt sich erhöhend auf die Zinsmarge eines Landes aus. Für beide Variablen gilt, daß sie protektionistisch verwendet werden können. Aufkäufe von Banken werden in der Regel von nationalen Aufsichts-, Wettbewerbsund Kartellbehörden begutachtet und müssen von ihnen freigegeben werden. Die Bewilligung oder Ablehnung eines Bankenkaufs ist somit vom Votum der inländischen Behörden abhängig. Ausländische Bewerbungen aus protektionistischen Gründen abzulehnen, ist also jederzeit möglich, die Entscheidungsträger müssen nur diskriminierend vorgehen. Wenn auch in der Regelung an sich zunächst keine Protektion erkennbar ist, so birgt die praktische Anwendung doch erheblichen Gestaltungsspielraum, der im protektionistischen Sinne mißbraucht werden kann. Entsprechend hoch ist das Protektionspotential dieser Variablen. Derartige Entscheidungen führen oftmals zu mehr oder minder versteckter Protektion, die argumentativ gerechtfertigt wird mit der Verhinderung des Ausverkaufs bedeutender Industrien. Eine Automatisierung der Genehmigungsverfahren in dem Sinne, daß die Zustimmung der verantwortlichen Behörden bei der Erfüllung der Anforderungen zwingend zu erfolgen hat und die Einzelfallentscheidungen ausschließt, würde dazu führen, daß ausländische Konkurrenten nicht länger diskriminiert werden könnten. 
- Nicht beschränkter Wertpapierhandel (d412): Hier finden die obigen Argumente erneut Anwendung. Das willkürlich erlassene Verbot des Wertpapierhandels nur für ausländische Banken stellt einen protektionistischen Eingriff dar.

\subsubsection{Erkenntnisse aus den Gesamtschätzungen}

Anhand der Gesamtschätzungen ist ablesbar, daß unterschiedliche Instrumente der Protektion in den beiden Untersuchungszeiträumen genutzt wurden. Für beide Zeiträume sind für die Bestimmung der Zinsmargen die Kontrollvariablen des Pro-Kopf-Einkommens und der Kapitalquoten der Banken von hoher Relevanz. Ebenfalls in beiden Zeiträumen von Bedeutung blieb die Variable „Wertpapierhandel“" (d412). Bei allen weiteren Regressoren hat ein vollständiger Austausch stattgefunden.

Im ersten getesteten Zeitraum war der Economic Freedom Indicator von hoher Relevanz. Die besten Ergebnisse ließen sich durch die explizite Betrachtung einzelner Indikatoren wie fiskalische Belastung, Geldpolitik oder Auslandsinvestitionen erzielen ( $F$-Wert: 17,66; adjustiertes $\mathrm{R}^{2}=0,7485$ ). Aber auch die Regression auf den Gesamtindikator ergab einen hohen F-Wert und gute Werte der Bestimmtheitsmaße (F-Wert: 15,36; adjustiertes $\mathrm{R}^{2}=0,6723$ ). Der Economic Freedom Indicator besaß hingegen im späteren Zeitraum (2001 - 2003) keinerlei statistische Signifikanz mehr.

Auffallend ist auch der niedrigere F-Wert $(9,15)$ sowie die geringeren Werte der Bestimmtheitsmaße $\left(R^{2}=0,5908\right.$; adjustiertes $\left.R^{2}=0,5262\right)$, die die Regression der zweiten Untersuchungsperiode erreichte. Die Ergebnisse stimmen im Hinblick auf die Signifikanzen mit den obig vorgenommenen Auswertungen überein, aber die herangezogenen Variablen können in diesem Zeitraum nur noch zu 52,62 Prozent die Zinsmarge erklären. Es müssen weitere, nicht bekannte Variablen hinzugekommen sein, die von erheblichem Einfluß auf die Zinsmargen sind. Legt man die Bestimmtheitsmaße zugrunde, so hat sich die Qualität der betrachteten Variablen als Determinanten der Nettozinsmargen, in einem Zeitraum von nur fünf Jahren, um erhebliche 14,61 Prozentpunkte verschlechtert.

Diese überraschend starke Veränderung in den Regressoren zwischen der ersten und der zweiten Gesamtschätzung zeigt, daß erhebliche Umwälzungen auf den Bankenmärkten stattgefunden haben.

Die Variablen, die ihren Einfluß in der Zeit vom ersten Untersuchungszeitraum zum zweiten verloren haben, sind:

- Beschränkung des Eigenkapitalbesitzes an Banken (d21),

- Nicht beschränkter Wertpapierhandel (d411), 
- Beschränkung des Besitzes von Nicht-Finanzunternehmen durch Banken (d443),

- Erfolgte Aufhebung von Beschlüssen des Bankenmanagements hinsichtlich Dividendenausschüttung, Bonuszahlung oder Gebührenhöhe durch die Aufsichtsbehörden (d116) sowie

- Economic Freedom Indicator.

Die ersten drei Variablen lassen sich unter dem Schlagwort „Beschränkung der Bankenaktivitäten" zusammenfassen. Diesen Variablen ist gemein, daß sie sehr leicht ersichtlich in den Members Schedule des GATS aufgefuhrt werden müssen und somit sofort als Protektion identifiziert werden. Hinzu tritt die Variable „Aufhebung von Managementbeschlüssen durch die Aufsichtsbehörden“, die ein weitreichendes Eingriffsrecht der Aufsichtsbehörden umschreibt.

Im späteren Untersuchungszeitraum spielten diese Variablen keine Rolle mehr, vielmehr traten als Regressoren der Zinsmarge verstärkt Aspekte der Kontrolle und Überwachung durch die Aufsichtsbehörden hervor wie:

- Pflichtmeldung der Bilanzprüfer bei der Aufsichtsbehörde bei Verdacht auf eine Straftat oder Hinterziehung (d56),

- Häufigkeit der Überprüfung mittlerer und kleinerer Banken durch die Aufsichtsbehörden (d125),

- Juristisches Vorgehen der Aufsichtsbehörden gegen fahrlässig handelnde Bilanzprüfer in den letzten 5 Jahren (d58),

- Mechanismen in Form von Unterlassungsanordnungen, die automatisch zu zivil- oder strafrechtlichen Sanktionen bei Fehlverhalten des Bankmanagements führen (d11) sowie die Variable

- Beschränkter Besitz von Geschäftsbanken durch Finanzunternehmen (d253).

Insbesondere der hohe Bedeutungsverlust des Economic Freedom Indicator zwischen den beiden Gesamtschätzungen ist auffallend. Als Maß des ökonomischen Freiheitsgrades hat er jeglichen Einfluß auf die Zinsmargen verloren und diskriminiert nicht mehr zwischen den Ländern - offensichtlich ein globaler Prozeß der Ubiquitärisierung wirtschaftlicher Freiheit.

Die Kapitalquote sowie der Wertpapierhandel stellen weiterhin mögliche Ansatzpunkte für die Politik dar, aber zunehmend gewinnt die Gestaltung der Aufsichtsbehörden und des Kontrollsystems an Bedeutung. Der Einfluß der Aufsichtsbehörden und der Kontrollbedingungen hat einen höheren Stellenwert für die Bestimmung der Zinsmargen erhalten als andere Aspekte des Bankengeschäfts. Die Überwachung der Banken und die Anforderungen der Aufsichtsbehörden gegenüber Bilanzprüfern sind bedeutende Determinanten der Zinsmargen geworden. 
Interessanterweise wurde eine Variable in den Gesamtschätzungen als Zinsmargendeterminante ermittelt, die in den Einzelschätzungen noch keinerlei Einfluß besaßen. Es handelt sich hierbei um die Existenz von Maßnahmen in Form von Unterlassungsanordnungen, die automatisch zu zivil- oder strafrechtlichen Sanktionen bei Fehlverhalten des Bankmanagements führen (d111).

Die Unterschiede in den Determinanten der Zinsmargen der beiden Untersuchungszeiträume bildet einen Wandel in der Entwicklung der Finanz- und Bankenmärkte $a b$, der sich innerhalb von nur wenigen Jahren vollzogen hat. Den genauen Einfluß der GATS 2000-Verhandlungen kann man nicht bestimmen. Es ist aber zu vermuten, daß derart weitreichende Veränderungen zu einem nicht unerheblichen Teil auf deren Beschlüsse zur weiteren Öffnung der Märkte zurückzuführen sind. Somit repräsentieren die Daten des jüngeren Zeitraums zum Teil das Ergebnis der Liberalisierungs- und Deregulierungsbemühungen durch die GATS-Verhandlungen. Sollen protektionistische Ziele verfolgt werden, so müssen sie ihren Niederschlag in den jeweiligen nationalen Kontrollmechanismen finden. Ein diskriminierendes Verhalten gegenüber ausländischen Banken aufrechtzuerhalten, wäre der einfachste Weg, um die heimischen Märkte gegenüber ausländischer Konkurrenz abzuschotten. Es bleibt des weiteren auch stets die Möglichkeit eines Verbots des Marktzutritts sowie der versteckten Diskriminierung.

Zum Abschluß dieses Vergleichs sei auf ein generelles Problem hingewiesen: Zinsmargen werden nicht einzig von protektionistisch verwendbaren Variablen determiniert. Sie repräsentieren eine Vielzahl weiterer Informationen. Auch liegen die empirischen Daten nicht in einer hinreichenden Detailtiefe für die Analyse vor. Dadurch ist es weder technisch noch ökonomisch möglich, den „Protektionsanteil" eindeutig herausfiltern.

In Kapitel 4.1.3 Variablengruppen und ihre Protektionspotentiale fuhrte die theoretische Auseinandersetzung mit dem potentiellen Protektionsgehalt der einzelnen Variablengruppen zur Konstruktion der Tabelle 4-1. Diese kann nun durch die in den Gesamtschätzungen ermittelten Determinanten der Zinsmarge ergänzt werden. 
Tab. 4-34: Determinanten der Zinsmargen - Protektionspotentiale in den Determinanten der Schätzungen

\begin{tabular}{|c|c|c|c|}
\hline Variablengruppen & Protektionspotentiale & 1. Untersuchungszeitraum & 2. Untersuchungszeitraum \\
\hline Kapitalquoten & Ja (durch Diskriminierung) & $\begin{array}{l}\text { Determinante der } \\
\text { Zinsmarge }\end{array}$ & $\begin{array}{l}\text { Determinante der } \\
\text { Zinsmarge }\end{array}$ \\
\hline $\begin{array}{l}\text { Attraktivität des Bankenmarktes } \\
\text { (Gewinnpotential) }\end{array}$ & $\mathrm{Ja}$ & & \\
\hline Geschäftsfelder der Banken & $\mathrm{Ja}$ & $\begin{array}{l}\text { Determinante der } \\
\text { Zinsmarge }\end{array}$ & $\begin{array}{l}\text { Determinante der } \\
\text { Zinsmarge }\end{array}$ \\
\hline Economic Freedom Indicator & $\mathrm{Ja}$ & $\begin{array}{l}\text { Determinante der } \\
\text { Zinsmarge }\end{array}$ & \\
\hline $\begin{array}{l}\text { Staatliche und ausländische } \\
\text { Besitzanteile } \\
\text { am Bankenmarkt }\end{array}$ & $\mathrm{Ja}$ & & \\
\hline Kontrollintensität & unter Umständen & & $\begin{array}{l}\text { Determinante der } \\
\text { Zinsmarge }\end{array}$ \\
\hline Liquidität (im weiteren Sinne) & unter Umständen & & \\
\hline Einlagensicherungssysteme & unter Umständen & & \\
\hline Verflechtungsgrad & unter Umständen & & $\begin{array}{l}\text { Determinante der } \\
\text { Zinsmarge }\end{array}$ \\
\hline
\end{tabular}


Tab. 4-34: Determinanten der Zinsmargen - Protektionspotentiale in den Determinanten der Schätzungen (Forts.)

\begin{tabular}{|c|c|c|c|}
\hline Variablengruppen & Protektionspotentiale & 1. Untersuchungszeitraum & 2. Untersuchungszeitraum \\
\hline $\begin{array}{l}\text { Anzahl der geschlossenen oder } \\
\text { fusionierten Banken }\end{array}$ & unter Umständen & & \\
\hline Befugnisse der Aufsichtsbehörde & unter Umständen & & \\
\hline Eigenkapital der Banken & unter Umständen & $\begin{array}{l}\text { Determinante der } \\
\text { Zinsmarge }\end{array}$ & \\
\hline Konzentrationsgrad & Nein & & \\
\hline $\begin{array}{l}\text { Rechnungslegung / } \\
\text { Rechnungsprüfung }\end{array}$ & Nein & & $\begin{array}{l}\text { Determinante der } \\
\text { Zinsmarge }\end{array}$ \\
\hline Ratings & Nein & & \\
\hline $\begin{array}{l}\text { Offenlegungspflichten / öffentliches } \\
\text { Monitoring }\end{array}$ & Nein & & \\
\hline $\begin{array}{l}\text { Haftbarkeit bzw. juristische } \\
\text { Verantwortung des } \\
\text { Bankenmanagements }\end{array}$ & Nein & $\begin{array}{l}\text { Determinante der } \\
\text { Zinsmarge }\end{array}$ & $\begin{array}{l}\text { Determinante der } \\
\text { Zinsmarge }\end{array}$ \\
\hline
\end{tabular}


Deutlich zu erkennen ist, daß die Determinantenstruktur der Zinsmarge in der zweiten Teilperiode zahlenmäßig größer und damit komplexer geworden ist. Gleichzeitig ist auch ihr Erklärungsvermögen für die Zinsmarge wesentlich gesunken, d.h. die Wirkung der Determinanten auf die Zinsmarge hat sich im Durchschnitt verringert. Somit haben in der zweiten Teilperiode Variablen an Bedeutung gewonnen, die von der vorliegenden Datenerhebung nicht erfaßt wurden.

Würden wir allen protektionsrelevanten Determinanten die gleiche Stärke an protektionistischer Wirkung zuschreiben, so läßt sich im Ergebnis festhalten, daß insgesamt der Grad an Protektion im Bankensektor rückläufig ist - falls nicht neue, noch unbekannte Determinanten der Zinsmargen hinzugetreten sind, die ebenfalls über Protektionsgehalt verfügen.

Unter Bezugnahme auf obige Tabelle 4-34 können wir unsere Überlegungen fortentwickeln und ein sehr tentatives Denkspiel vollziehen: Vorausgesetzt, die Protektionswirkung der einzelnen Variablen entspricht ihrem in den Regressionsgleichungen ermittelten Werten, dann erreicht das Erklärungsvermögen der Determinanten im ersten Untersuchungszeitraum einen $R^{2}$-Wert von 0,7485 . Die bedeutet, daß im Durchschnitt jede der protektionistisch wirkenden Determinanten einen Einfluß in Höhe von 0,1497 erreicht. Analog ergibt sich für den späteren Untersuchungszeitraum ein Wert von 0,0878. Die erste Periode weist drei Determinanten auf, denen wir eine starke Signifikanz und damit einen hohen Grad an Protektionswirkung zuschreiben. Wir gewichten sie folglich mit dem Wert von 0,1497. Zwei weitere Determinanten erhielten das Attribut „Protektionspotential: unter Umständen“, diese gewichten wir zu 50 Prozent $(0,0746)$, Determinanten ohne Protektionsbezug erhalten ein Gewicht von Null. Die Summe dieser Gewichtung nimmt den Wert von 0,522 ein und ist folgendermaßen zu interpretieren. Bei einem 100\%igen Protektionsgehalt der „Ja“-Gruppe und einem 50\%igen Protektionsgehalt der „Unter-Umständen“-Gruppe werden rein kalkulatorisch durch den Protektionsgehalt der entscheidenden Variablengruppen ca. 52\% der Varianz der Zinsmarge erklärt.

Ein analoges Vorgehen für die zweite Periode ergibt als Summe der Gewichtung einen Wert von 0,1317. In diesem Fall werden nur ca. 13\% der Varianz der Zinsmarge durch den Protektionsgehalt der entscheidenden Variablen bestimmt. Die absolute Höhe dieser Erklärungsanteile sagt natürlich aufgrund ihres artifiziellen Charakters wenig aus, jedoch sind die Relationen der Perioden interpretierbar: Während der Protektionsgehalt der Variablen in der ersten Teilperiode noch knapp $70 \%$ des Bestimmtheitskoeffizienten erklären kann, so ist das in der zweiten Periode nur noch zu 25\% der Fall. Es besteht also die begründete Vermutung, daß mit dem abnehmenden Erklärungsgehalt der vorliegenden Variablen in der Zeit auch der Erklärungsgehalt protektionistisch verwendbarer Variablen abnimmt und dies zudem überproportional. Wenn man weiterhin davon ausgeht, daß die Motivation der Regierungen immer in der Ausreizung der ohne 
Retaliation-Effekte realisierbaren Protektion besteht, dann dürfte dieses artifizielle Bild nicht sonderlich weit von der Realität entfernt sein.

Weitere Schlußfolgerungen lassen sich aus den vorliegenden Daten nicht herleiten. Wie bei jeder empirischen Erhebung, die auf die Questionaire-Technik zurückgreift, müssen die Motive und Ziele der Befragten betrachtet werden. Eine Offenlegung des Protektionsniveaus und der praktizierten Protektionstechniken ist nicht im Interesse der Länder. Sie würden ihre Position bei internationalen Verhandlungen schwächen und angreifbar werden. Für den weiteren Erkenntnisgewinn hinsichtlich der Fragestellung muß in Zukunft wohl stärker die qualitative Forschung in den Mittelpunkt rücken. 


\section{$5 \quad$ Fazit}

Der GATS-Vertrag dient als internationaler Verhandlungsrahmen zur Reduktion von Protektion. Er hat viel zur Transparenz und Offenlegung der von Land zu Land sehr unterschiedlichen Bestimmungen beigetragen, denn er dokumentiert die existierenden Handelshemmnisse in ihren Häufigkeiten und vielfältigen Ausprägungen. Die Bankenmärkte vieler Länder sind immer noch gegenüber ausländischen Konkurrenten abgeschottet. Der Finanzsektor gilt als eine Schlüsselbranche - gerade die Entwicklungsländer wehren sich z.T. vehement gegen eine Öffnung dieser Märkte. Da mag eine gewisse Angst vor Überfremdung der heimischen Wirtschaft vorherrschen, sicherlich werden aber handfeste wirtschaftliche Interessen einflußreicher Gruppen bedroht gesehen.

Derzeit sind die Entwicklungs- und Schwellenländer weitaus schlechter in die internationalen Finanzmärkte integriert als die Industrienationen. Entsprechend profitieren fast ausschließlich nur die Industriestaaten von den Vorzügen eines globalisierten Kapitalmarktes. Die ärmeren Länder fürchten in der Mehrheit, die Kontrolle über ihre heimischen Finanzmärkte an international agierende, finanzkräftige Banken zu verlieren. Sie bewahren hinter einer Mauer aus Protektion den aktuellen Status quo und verschenken damit die Chance auf eine zügige ökonomische Entwicklung ihrer Länder. Daß es trotz dieser Konstellation gelang, in den letzten Jahren protektionistische Hürden abzubauen, ist sicherlich zu einem Gutteil den Verhandlungsrunden im Rahmen des GATS und dem stetigen Eintreten der WTO für Liberalisierungen zu verdanken.

Die Verwendung von Zinsmargen als Indikator der Protektion erweist sich als gangbarer Weg. Sie reflektieren neben den volkswirtschaftlichen Rahmenbedingungen auch die Wirkung der Protektion und weisen den Vorteil auf, daß sie am Markt gebildet werden und somit als Preis fungieren. Den Banken kommt in ihrer Funktion als Finanzintermediär in der Volkswirtschaft eine hohe Bedeutung bei der optimalen Allokation von Geldmitteln und damit des Produktionsfaktors Kapital zu. Der aktive Gebrauch protektionistischer Maßnahmen wird von einer Volkswirtschaft teuer, nämlich in Form hoher Zinsmargen erkauft, mittels derer der Bankensektor die Protektion einpreist. Der Einfluß auf das Sparverhalten der Haushalte und insbesondere auf das Investitionsverhalten der Unternehmen ist nicht zu unterschätzen. Ausbleibende Investitionen verringern das Produktionspotential der Volkswirtschaft und wirken sich negativ auf ihr zukünftiges Wachstum aus.

Diejenigen Länder, die sich durch eine hohe Regulierungsdichte und die Tendenz zur Abschottung gegenüber ausländischen Banken auszeichnen, weisen erwartungsgemäß auch die deutlich höheren Zinsmargen auf. Die entwickelten und die aufstrebenden Industrienationen haben hingegen relativ niedrige Zinsmargen aufzuweisen. Abgesehen von wenigen Ausnahmen ist eine Tendenz zur Senkung der Zinsmargen, eindeutig zu beobachten. 
Die Industrienationen haben innerhalb von nur fünf Jahren eine Reduktion der Zinsmargen um annähernd 33 Prozent erreicht, zugleich weisen sie auch hohe Wachstumsraten des Pro-Kopf-Einkommens auf (23 Prozent). Auch viele ärmere Länder haben erhebliche Anstrengungen unternommen, um ihre Märkte zu öffnen und ihre Zinsmargen zu senken - jedoch verharrt in diesen Ländern die Zinshöhe auf einem vergleichsweise hohen Niveau. Während in der Klasse der Pro-KopfEinkommen von 1.000-4.999 US-Dollar die radikalste Senkung der Zinsmargen um 39 Prozent erreicht wurde, konnten die Länder mit einem Pro-KopfEinkommen von unter 1.000 US-Dollar dieselben nur um 17 Prozent senken. Der Wille oder Druck zur Liberalisierung, wenn auch nur zu einer schrittweisen, scheint zumindest vorhanden zu sein - wenn auch mit unterschiedlichem Erfolg.

Generell sind die Zinsmargen innerhalb der untersuchten Perioden im Durchschnitt stark gesunken. Bezieht man in die Betrachtung alle Länder ein, so ist die Nettozinsmarge im Durchschnitt von 0,0474 auf 0,0399 gesunken. In der zweiten Periode wurden also im Schnitt nur noch 82 Prozent der im ersten Zeitraum angesetzten Zinsmargen realisiert. Betrachtet man die Zahlen nur für die Länder, die in beiden Zeiträumen Daten zur Verfügung stellten, so ist der Unterschied noch deutlicher: Die Nettozinsmargen verringerten sich von 0,0484 auf 0,0334; das entspricht einer Reduktion der Nettozinsmargen um nahezu 31 Prozent. Der Wettbewerbsdruck hat sich im Verlauf von nur wenigen Jahren also erheblich erhöht und die Banken zu einer Abschmelzung ihrer Zinsmargen gezwungen.

Die über diese Datenanalyse hinausgehende multivariate Analyse zeigt, daß es nicht nur in der Höhe der Protektion Veränderungen gab, sondern sich auch eine Art Strukturwandel hinsichtlich der protektionistischen Maßnahmen vollzogen hat. Für die Determinierung der Zinsmargen gewannen die Kontroll- und Prüfverfahren der Aufsichtsbehörden erheblich an Bedeutung. Je intensiver und exakter die Kontrollen ausgeübt wurden, um so geringer fallen die Zinsmargen der Länder aus.

Die Bedeutung individuell gestaltbarer Variablen und Instrumente (wie bspw. die Eigenkapitalbeschränkung) wurde erheblich reduziert. Dafür rückten vermehrt die Kontrollen und Bestimmungen der Aufsichtsorgane in den Vordergrund der Protektionsgestaltung. Eine mögliche Erklärung für den Bedeutungswechsel der Determinanten kann in dem unterschiedlichen Grad der Offensichtlichkeit liegen. Regulierungen, die sich leicht ersichtlich gegen ausländische Banken richten, werden sehr schnell als Verhandlungspunkte identifiziert. Wird die Protektion aber verklausuliert, so sinkt die Entdeckungs- und anschließende Verhandlungswahrscheinlichkeit erheblich. Um ihr Protektionsniveau beibehalten zu können, müssen interessierte Länder entsprechende Maßnahmen verschleiern. Der Wandel in der Qualität der Determinanten ist als Resultat der verstärkten Internationalisierung des Bankengeschäfts und der stärkeren Verflechtung der Finanzmärkte zu interpretieren. Der Druck zur Liberalisierung der eigenen Bankenmärkte wuchs in den letzten Jahren erheblich. Die Industrienationen drängten und drängen inner- 
halb der WTO-Verhandlungen vehement und stetigen Druck ausübend auf weitere Liberalisierungsmaßnahmen.

Auch die Verhandlungsmethoden innerhalb des GATS-Rahmen bedürfen einer Reform. Die Verhandlungen sind zu aufwendig, finden meist bilateral statt und sind sehr zeitintensiv. Eine Umstellung auf Verhandlungen, die sich auf die horizontale Ebene beziehen, würde sicherlich die Verfahren entlasten, beschleunigen und vereinfachen. Die Liberalisierungserfolge würden automatisch auf alle Dienste einer Kategorie übertragen - damit unterblieben langwierige Verhandlungen über die Handelsbedingungen jeder einzelnen Dienstleistung. Auch sollte ein Wechsel vom Ansatz der Positivlisten hin zur Negativliste erfolgen. Darin würden nur die geschützten Dienstleistungssektoren erfaßt und konkret über deren Deregulierung diskutiert werden. Bei der Positivliste ist noch der Schritt der Erfassung aller Sektoren davor geschaltet mit den jeweiligen Angaben, ob ausländischen Unternehmen die Betätigung erlaubt wird (und wenn ja, zu welchen Bedingungen) oder nicht.

Aus wirtschaftswissenschaftlicher Sicht sind alle Liberalisierungsversuche des Handels und die Annäherung an eine Freihandelssituation als positiv zu bewerten. Dadurch werden die wahren Preise offengelegt, die Allokation des Produktionsfaktors Kapital verbessert und die Weltwohlfahrt maximiert. Die Finanzmärkte gelten, trotz der jüngsten Deregulierungsfortschritte, immer noch als stark reglementiert. Die Handelshemmnisse und ihre kreativen Weiterentwicklungen müssen beobachtet werden und zusätzliche Deregulierungsbemühungen unternommen werden.

Insbesondere die Entwicklungsländer müssen zu einer Öffnung ihrer Bankenmärkte motiviert und zur Teilnahme an den GATS-Verhandlungen angehalten werden - dank des momentan hohen Schutzes, den sie ihren Bankensektoren gewähren, haben sie auch die höchsten Deregulierungsgewinne zu erwarten.

Im Hinblick auf die Determinanten der Zinsmargen, wäre für die Zukunft auch zu prüfen, wie sich der deutliche Verlust an Erklärungsgehalt interpretieren läßt. Laut der geprüften Daten erklärten im ersten Untersuchungszeitraum die verschiedenen getesteten Variablen noch zu annähernd 75 Prozent die Variationen in den Zinsmargen. Nur zwei Jahre später lag der Erklärungsgehalt bei nur noch ca. 53 Prozent. Durch Analyse des vorliegenden Datenmaterials konnte nicht geklärt werden, worauf der Bedeutungsverlust beruht. Diese fehlenden Faktoren zu ermitteln, ist von grundlegender Bedeutung für weitere Forschung in diesem Bereich. Eine Möglichkeit könnte der Einbezug von detaillierten, betriebswirtschaftlichen Daten der Banken sein. Insbesondere Faktoren wie die Gesamtkostenhöhe einer Bank, die Kosten-Gewinn-Relation, die wachsende Bedeutung der Gebühreneinnahmen als Gewinnquelle, die Risikobereitschaft des Managements, vollzogene oder geplante Fusionen (Akquisitionen) könnten von Bedeutung sein. Angesichts eines abnehmenden Protektionsgrades stehen die Banken international in einem verschärften Wettbewerb zueinander. Die betriebswirtschaftliche Effizienz einer 
Bank wird nach Wegfall des Schutzes vordringlicher und könnte von zunehmender Bedeutung für die Erklärung der Zinsmargen sein. 


\section{Anhang}

\section{A1: Legende der Variablen}

\section{Entry into Banking}

d010 Number of banks

d011 Is information on source of funds for capital required? yes $=1$, no $=0$

d012 Are the sources of funds to be used as capital verified by authorities?

d015 Can borrowed funds be used?

yes $=1$, no $=0$

Which of the following are legally required to be submitted before issuance of the banking license?

d0161 Draft by-laws

yes $=1$, no $=0$

d0162 Intended organization chart

yes $=0$, no $=1$

d0163 First 3-year financial projections

yes $=1$, no $=0$

d0164 Financial information on shareholders

yes $=1$, no $=0$

d0165 Background/experience of future directors

yes $=1$, no $=0$

d0166 Background/experience of future managers

yes $=1$, no $=0$

d0167 Sources of funds in capitalization of new bank

yes $=1$, no $=0$

d0168 Intended market differentiation of new bank

yes $=1$, no $=0$

d0171 Number of domestic applications for banking licenses

d0172 Number denied

d0181 Number of foreign applications for banking licenses

d0182 Number denied

Basis for denial of appilications

d0191 Capital amount/quality

$$
\begin{aligned}
& \text { yes }=1, \text { no }=0 \\
& \text { yes }=1, \text { no }=0 \\
& \text { yes }=1, \text { no }=0 \\
& \text { yes }=1, \text { no }=0
\end{aligned}
$$

d0193 Reputation

d0194 Incomplete application

Number of applications from foreign entities to enter through the acquisition of domestic bank?

d0195 ....Received

d01951 ....Denied

Number of applications from foreign entities to enter through new, capitalized subsidiary?

d0196 ....Received

d01961 ....Denied 
Number of applications from foreign entities to enter through opening a branch?

d0197 ....Received

d01971 ....Denied

Number of applications from foreign entities to enter through some other means?

d0198 ....Received

d01981 ....Denied

\section{Ownership}

$\mathrm{d} 21$

$$
\text { owned }
$$

by single owner?

$\mathrm{d} 211$ If yes, what is it?

d22 Can related parties own capital in a bank?

d221 If yes, what is the maximum percentage?

Regulatory restrictiveness of ownership by nonfinancial firms of banks

d231

d232

d233

d234

d24

d251

d252

d253

d254

d27

d28 ....permitted

....unrestricted

....restricted

....prohibited

What fraction of capital in the largest 10 banks is

owned by commercial/industrial and/or financial

conglomerates? If there are fewer than 10 banks, use

that number in your answer.

Can non-bank financial firms (e.g. insurance companies, finance companies, etc.) own commercial banks?

....permitted

....unrestricted

....restricted

....prohibited

Of commercial banks in your country, what fraction of: deposits is held by the five (5) largest banks at year-end 2001?

Of commercial banks in your country, what fraction of: assets is held by the five (5) largest banks at yearend 2001? yes $=1$, no $=0$

yes $=1$, no $=0$

$$
\begin{aligned}
& \text { yes }=1, \text { no }=0 \\
& \text { yes }=1, \text { no }=0 \\
& \text { yes }=1, \text { no }=0 \\
& \text { yes }=1, \text { no }=0
\end{aligned}
$$

$$
\begin{aligned}
& \text { yes }=1, \text { no }=0 \\
& \text { yes }=1, \text { no }=0 \\
& \text { yes }=1, \text { no }=0 \\
& \text { yes }=1, \text { no }=0
\end{aligned}
$$


Of all deposit-taking institutions in your country, what

d29 fraction of their assets is held by just commercial banks?

\section{Capital}

d31 Minimum capital-asset ratio requirement (\%)

d311 Is it risk-weighted in line with Basle guidelines? $\quad$ yes $=0$, no $=1$

d32 Does the minimum ratio vary as a function of an individual bank's credit risk?

yes $=0$, no $=1$

d33

Does the minimum ratio vary as a function of market risk?

yes $=0$, no $=1$

What is the actual risk-adjusted capital ratio in banks

d34 as of year-end 2001, using the 1988 Basle Accord $\quad$ yes $=0$, no $=1$ definitions?

d342 What is the actual capital ratio (i.e., not risk-adjusted)

of banks as of year-end 2001?

d35 Is subordinated debt allowable (required) as part of capital?

yes $=1$, no $=0$

d36 What fraction of revaluation gains is allowed as part of capital?

d37 Percentage of banking system's assets in banks that are $50 \%$ or more government owned.

d38 Percentage of banking system's assets in banks that are $50 \%$ or more foreign owned.

Before minimum capital adequacy is determined Are deducted from capital...

d391 ....Market value of loan losses?

yes $=0$, no $=1$

d392 ....Unrealized securities losses?

yes $=0$, no $=1$

d393

....Unrealized foreign exchange losses?

yes $=0$, no $=1$

\section{Activities}

What is the level of regulatory restrictiveness for bank participation in .... activities?

Securities

d411 ....permitted

yes $=1$, no $=0$

$\mathrm{d} 412 \quad$....unrestricted

yes $=1$, no $=0$

d413 ....restricted

yes $=1$, no $=0$

$\mathrm{d} 414$

....prohibited

yes $=1$, no $=0$

Insurance 
yes $=1$, no $=0$

d422 ...unrestricted

yes $=1$, no $=0$

d423 ....restricted

yes $=1$, no $=0$

d424

...prohibited

yes $=1$, no $=0$

Real estate

d431 ....permitted

yes $=1$, no $=0$

d432 ....unrestricted

yes $=1$, no $=0$

d433 ....restricted

yes $=1$, no $=0$

d434

....prohibited

yes $=1$, no $=0$

Bank Owning Nonfinancial Firms

d441 ....permitted

yes $=1$, no $=0$

d442 ....unrestricted

yes $=1$, no $=0$

d443 ....restricted

yes $=1$, no $=0$

d444

....prohibited

yes $=1$, no $=0$

\section{External Auditing Requirements}

d51 Is an external audit compulsory?

yes $=0$, no $=1$

d52

Are there specific requirements for the extent of audit?

d53 Are auditors licensed or certified?

yes $=0$, no $=1$

d54 Is auditor's report given to supervisory agency?

yes $=0$, no $=1$

Can supervisors meet external auditors to discuss

d55 report

yes $=0$, no $=1$

without bank approval?

yes $=0$, no $=1$

Are auditors required by law to communicate directly

d56 to the supervisory agency any presumed involvement of bank directors or senior managers in illicit

yes $=0$, no $=1$ activities, fraud, or insider abuse?

d57

Can legal action against external auditors be taken by supervisor for negligence?

yes $=0$, no $=1$

d58

Has legal action been taken against an auditor in last 5 yes $=0$, no $=1$ years?

\section{Internal Management/Organizational Requirements}

Can supervisors force banks to change internal organizational structure?

yes $=0$, no $=1$

d62 Has this power been utilized in last 5 years?

yes $=0$, no $=1$ 
Are there explicit, verifiable, and quantifiable guide-

lines regarding asset diversification? (for example, are

d71 banks required to have some minimum diversification yes $=0$, no $=1$

of loans among sectors, or are their sectoral concentration limits)?

d72 Are banks prohibited from making loans abroad? yes $=1$, no $=0$

Do Minimum liquidity requirement exist? Are banks

d73 required to hold either liquidity reserves or any $\quad$ yes $=0$, no $=1$

deposits at the Central Bank?

Minimum liquidity requirement

d731 ....Min.

d732 ....Max.

d74 Is interest paid on reserves or any kind of compensation?

Are banks allowed to hold reserves in foreign denomi-

d753 nated currencies or other foreign denominated instru- yes $=0$, no $=1$ ments?

d754

What percent of the commercial banking system's assets is foreign-currency denominated?

\section{Depositor (Savings) Protection Schemes}

\begin{tabular}{|c|c|c|}
\hline d81 & Is there an explicit deposit insurance scheme? & yes $=0$, no $=1$ \\
\hline $\mathrm{d} 82$ & Is it funded by the government, banks or both? & $\begin{array}{l}\text { govern. or both } \\
=1, \text { banks }=0\end{array}$ \\
\hline 83 & $\begin{array}{l}\text { Ratio of accummulated funds to total bank assets. If } \\
\text { pre-funded, what is the ratio of accumulated funds to } \\
\text { total bank assets? }\end{array}$ & $1=100 \%$ \\
\hline d84 & Is there a insurance limit per person? & yes $=1$, no $=0$ \\
\hline 35 & $\begin{array}{l}\text { Does deposit insurance authority make the decision to } \\
\text { intervene a bank? }\end{array}$ & yes $=0, n$ \\
\hline
\end{tabular}

As a share of total assets, what is the value of large

d86 denominated debt liabilities of banks-subordinated debt, bonds, etc.-that are definitely not covered by any explicit or implicit savings protection scheme?

Number of banks merged/closed (as part of failure resolution) in last 5 years.

d88 Were depositors wholly compensated the last time a bank failed?

yes $=1$, no $=0$ 
Can deposit insurance agency take legal action against yes $=1$, no $=0$ bank directors/officials?

\section{Provisioning Requirements}

d91 Is there a formal definition of "non-performing loan"? yes =0, no =1
d93 What is the ratio of non-performing loans to total
assets as of year-end 2001?
d94 If one loan is non-performing, are other loans of a
multiple-loan customer classified as non-performing? yes = 0, no = 1
d95 What is the aggregate net interest margin-to-asset ratio
as of year-end 2001?
d96 What is the aggregate overhead costs-to-asset ratio as
of year-end 2001?

\section{Accounting/Information Disclosure Requirements}

d101 Does income statement contain accrued but unpaid yes $=0$, no $=1$

d102 Does income statement contain accrued but unpaid $\quad$ yes $=0$, no $=1$

d103 Are off-balance sheet items disclosed to supervisors? yes $=0$, no $=1$

d104 Are off-balance sheet items disclosed to public? yes $=0$, no $=1$
d105 Must banks disclose risk management procedures to public?
yes $=0$, no $=1$

d106 Are directors legally liable for erroneous/misleading information?

d107 Have penalties been enforced?

yes $=0$, no $=1$

yes $=0$, no $=1$

d108 Do regulations require credit ratings for commercia
banks?
Percentage of the top ten banks that are rated by
d109 international credit rating agencies (e.g. Moody's international credit ratin

d1101 Which bank activities are rated? Bonds issuance? yes $=0$, no $=1$

d1102 Which bank activities are rated? Commercial Paper? yes $=0$, no $=1$

Other activity (e.g., issuance of bank certificates of
d1103 deposit, pension and mutual funds, insurance companies, financial guarantees, etc.)?
yes $=0$, no $=1$




\section{Discipline/Problem Institutions/Exit}

Are there any mechanisms of cease-desist type orders

d111 whose infraction leads to automatic imposition of civil yes $=0$, no $=1$ $\&$ penal sanctions on banks directors \& managers?

Can the supervisory agency order directors/manage-

d112 ment to constitute provisions to cover actual/potential yes $=0$, no $=1$ losses?

Can the supervisory agency suspend director's decision to distribute:

d113 ....dividends

d114 ....bonuses?

d115 ....management fees?

d116 Any such actions taken in last 5 years?

yes $=0$, no $=1$

yes $=0$, no $=1$

yes $=0$, no $=1$

yes $=0$, no $=1$

d117 Can the supervisory agency supercede bank shareholder rights and declare bank insolvent?

yes $=0$, no $=1$

Does banking law allow supervisory agency to sus-
d118 send some or all ownership rights of a problem bank?

yes $=0$, no $=1$

Does the law establish pre-determined levels of sol-

d119 vency deterioration which forces automatic actions yes $=0$, no $=1$ such as intervention?

Regarding bank restructuring \& reorganization, can supervisory agency or any other governmental agency do the following:

d1110 …supercede shareholder rights.

yes $=0$, no $=1$

d1111 ....remove and replace management.

yes $=0$, no $=1$

d1112 ....remove and replace directors.

yes $=0$, no $=1$

d1113

....forbear certain prudential regulations.

yes $=0$, no $=1$

d1114

Insure liabilities beyond any explicit deposit insurance scheme?

yes $=0$, no $=1$

d1115 Number of banks closed in last 5 years.

d1116

Percentage of total bank assets accounted for by these banks.

\section{Supervision}

\begin{tabular}{ll}
\hline $\mathrm{d} 121$ & $\begin{array}{l}\text { Is there more than one supervisory body? } \\
\text { How many professional bank supervisors are there in } \\
\text { total? }\end{array}$ \\
$\mathrm{d} 122$ & yes $=0, \mathrm{no}=1$ \\
$\mathrm{~d} 124$ & $\begin{array}{l}\text { Number of onsite examinations per bank in last } 5 \\
\text { years }\end{array}$
\end{tabular}


d125 How frequently are onsite inspections conducted in large and medium size banks?

$$
\begin{aligned}
& \text { annually }=1, \\
& \text { every two year } \\
& \text { or more }=0
\end{aligned}
$$

d126 Average tenure of a supervisor (years)

$\begin{array}{lll}\text { d128 } & \begin{array}{l}\text { Must infraction of any prudential regulation found by } \\ \text { a supervisor be reported? }\end{array} & \text { yes no }=1 \\ \text { d129 } & \text { Any mandatory actions in these cases? } & \text { yes }=0, \text { no }=1 \\ \text { d1210 Are supervisors legally liable for their actions? } & \text { yes }=0, \text { no }=1\end{array}$


A2: Länder der Untersuchung

\begin{tabular}{l|l}
$\begin{array}{l}\text { Daten aus beiden Perioden liegen } \\
\text { vor für die Länder: }\end{array}$ & $\begin{array}{l}\text { Länder, für die nur in einem Unter- } \\
\text { suchungszeitraum Daten vorliegen: }\end{array}$ \\
\hline Australia & Albania \\
Austria & Algeria \\
Belgium & Antigua and Barbuda \\
Bolivia & Argentina \\
Botswana & Azerbaijan \\
Burundi & Bahrain \\
Canada & Belize \\
Chile & Benin \\
Croatia & Bhutan \\
Cyprus & Bosnia and Herzegovina \\
Czech Republic & Brazil \\
Egypt & Bulgaria \\
Estonia & Burkina Faso \\
Finland & Cambodia \\
Germany & Colombia \\
Ghana & Commonwealth of Dominica \\
Greece & Costa Rica \\
Honduras & Côte d'Ivoire \\
Hungary & Ecuador \\
Iceland & El Salvador \\
India & Fiji \\
Israel & Grenada \\
Italy & Guatemala \\
Jordan & Guinea \\
Kenya & Guinea Bissau \\
Latvia & Guyana \\
Lebanon & Hong Kong, China \\
Lithuania & \\
Luxembourg & Kazakstan \\
\hline
\end{tabular}




\begin{tabular}{|c|c|}
\hline $\begin{array}{l}\text { Daten aus beiden Perioden liegen } \\
\text { vor für die Länder: }\end{array}$ & $\begin{array}{l}\text { Länder, für die nur in einem Unter- } \\
\text { suchungszeitraum Daten vorliegen: }\end{array}$ \\
\hline Macedonia & Macau, China \\
\hline Malta & Madagascar \\
\hline Mauritius & Malaysia \\
\hline Mexico & Mali \\
\hline Moldova & Nicaragua \\
\hline Morocco & Niger \\
\hline Netherlands & Norway \\
\hline New Zealand & Oman \\
\hline Nigeria & Pakistan \\
\hline Peru & Panama \\
\hline Philippines & Papua New Guinea \\
\hline Poland & Paraguay \\
\hline Romania & Portugal \\
\hline Russia & Saint Kitts and Nevis \\
\hline Rwanda & Saint Lucia \\
\hline Singapore & Saint Vincent and The Grenadines \\
\hline Slovenia & Samoa (Western) \\
\hline South Africa & Saudi Arabia \\
\hline Spain & Senegal \\
\hline Sweden & Serbia and Montenegro \\
\hline Switzerland & Seychelles \\
\hline Taiwan & Singapore \\
\hline Thailand & Slovakia \\
\hline Trinidad and Tobago & South Korea \\
\hline \multirow[t]{6}{*}{ United States } & Suriname \\
\hline & Swaziland \\
\hline & Tajikistan \\
\hline & Togo \\
\hline & Tonga \\
\hline & Tunisia \\
\hline
\end{tabular}




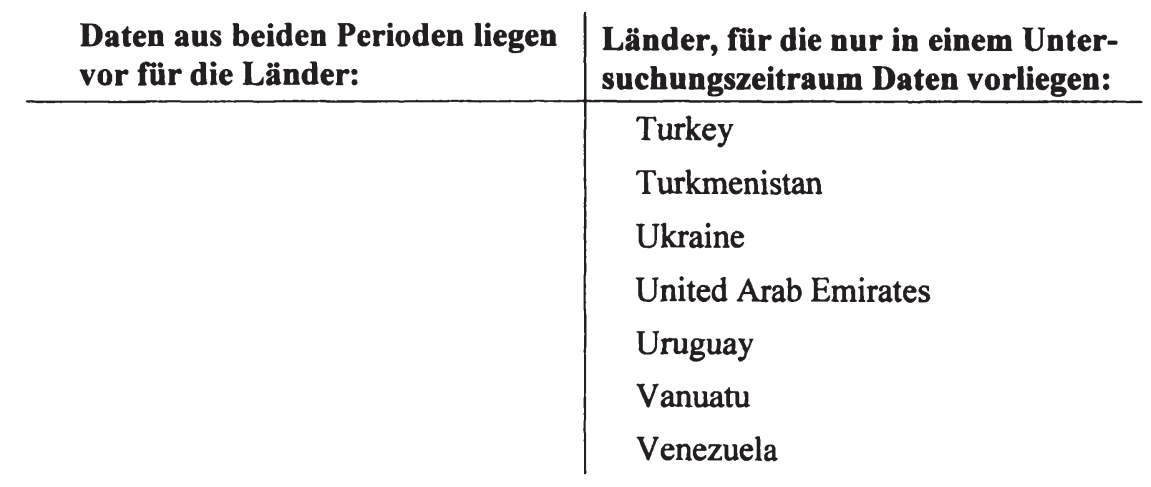

Ausschlaggebend für die Berücksichtigung eines Landes in der Regression ist die Verfügbarkeit der Variablen „Nettozinsmarge“. 


\section{A3: Zinsmargen und Pro-Kopf-Einkommen}

Die Periode 1998 - 2000

\begin{tabular}{|c|c|c|c|c|c|}
\hline Land & NIM & $\mathbf{P K E} \mathbf{E}^{1}$ & Land & NIM & PKE \\
\hline Australia & 0,032 & 20,28465 & Kuwait & 0,0187 & 16,90577 \\
\hline Austria & 0,0216 & 23,76565 & Latvia & 0,0692 & 3,25978 \\
\hline Bahrain & 0,0245 & 11,89655 & Lebanon & 0,0418 & 3,83373 \\
\hline Bangladesh & 0,0205 & 0,34738 & Lithuania & 0,0843 & 3,24721 \\
\hline Belarus & 0,1068 & 1,14115 & Luxembourg & 0,0119 & 44,75677 \\
\hline Belgium & 0,0238 & 22,26839 & Macedonia & 0,0957 & 1,77042 \\
\hline Bolivia & 0,0562 & 1,00972 & Malta & 0,0228 & 9,76079 \\
\hline Botswana & 0,0703 & 3,13483 & Mauritius & 0,0391 & 3,72692 \\
\hline Burundi & 0,1074 & 0,09968 & Mexico & 0,057 & 5,935 \\
\hline Canada & 0,0203 & 23,19801 & Moldova & 0,1003 & 0,30126 \\
\hline Chile & 0,0502 & 4,9644 & Mor & 0,0501 & 1,16126 \\
\hline atia & 0,054 & 4,20713 & Namibia & 0,0664 & 1,80229 \\
\hline Cyprus & 0,0253 & 12,08344 & Nepal & 0,0541 & 0,23843 \\
\hline Czech Republic & 0,0288 & 5,42255 & Netherlands & 0,0197 & 23,28277 \\
\hline Denmark & 0,0528 & 29,63032 & New Zeal & 0,0303 & 13,52448 \\
\hline Egypt & 0,0242 & 1,55414 & Nigeria & 0,0894 & 0,33156 \\
\hline Estonia & 0,0615 & 3,98689 & Panama & 0,0286 & 4,07165 \\
\hline Finland & 0,0199 & 23,18351 & Peru & 0,0792 & 2,04656 \\
\hline France & 0,0286 & 22,21657 & Philippines & 0,0456 & 0,99068 \\
\hline Germany & 0,0266 & 22,75001 & Poland & 0,0681 & 4,30937 \\
\hline Ghana & 0,1161 & 0,25405 & Romania & 0,0845 & 1,65097 \\
\hline Greece & 0,035 & 10,26794 & Russia & 0,0599 & 1,78426 \\
\hline Honduras & 0,0882 & 0,92346 & Rwanda & 0,0574 & 0,2349 \\
\hline Hungary & 0,0486 & 4,65688 & Singapore & 0,0286 & 22,76658 \\
\hline Iceland & 0,0415 & 29,96319 & Slovenia & 0,04 & 9,58629 \\
\hline India & 0,0348 & 0,45021 & South Africa & 0,0622 & 3,01995 \\
\hline Indonesia & 0,0562 & 0,80004 & Spain & 0,034 & 13,87068 \\
\hline Ireland & 0,0349 & 24,84836 & Sri Lanka & 0,0448 & 0,88438 \\
\hline Israel & 0,0322 & 18,35779 & Sweden & 0,0239 & 27,0118 \\
\hline Italy & 0,0367 & 18,62998 & Switzerland & 0,0175 & 34,29531 \\
\hline Jamaica & 0,0943 & 2,87361 & Taiwan (Chin.) & 0,0242 & 13,8797 \\
\hline Japan & 0,0207 & 37,40891 & Thailand & 0,023 & 2,0209 \\
\hline Jordan & 0,0347 & 1,72855 & $\begin{array}{l}\text { Trinidad \& } \\
\text { Tobago }\end{array}$ & 0,0468 & 6,32608 \\
\hline & $0,0.0$ & 0,3474 & Unit. Kingdom & 0,0298 & 24,44545 \\
\hline Korea & 0,0239 & 10,89024 & United States & 0,0434 & 34,59947 \\
\hline
\end{tabular}

\footnotetext{
${ }^{1}$ Pro-Kopf-Einkommen in Tsd. US-Dollar.
} 
Die Periode 2001 - 2003

\begin{tabular}{|c|c|c|c|c|c|}
\hline Land & NIM & PKE & Land & NIM & PKE \\
\hline Albania & 0,029 & 1,8002 & Greece & 0,0251 & 15,60801 \\
\hline Algeria & 0,0064 & 2,136759 & Grenada & 0,0461 & 4,18164 \\
\hline $\begin{array}{l}\text { Antigua \& } \\
\text { Barbuda }\end{array}$ & 0,0004 & 9,662246 & Guatemala & 0,052 & 2,00940 \\
\hline Argentina & 0,148 & 3,422147 & Guinea & 0,057 & 0,45903 \\
\hline Australia & 0,0279 & 26,27521 & Guinea Bissau & 0,08 & 0,16025 \\
\hline Austria & 0,014 & 31,28876 & Guyana & 0,011 & 0,96429 \\
\hline Azerbaijan & 0,054 & 0,86694 & Honduras & 0,0597 & 0,98558 \\
\hline Bahrain & 0,0194 & 13,49888 & Hong Kong, & 0,02 & 22,75864 \\
\hline Belgium & 0078 & 29,09562 & Hung & 0,040 & 8,17345 \\
\hline Belize & 0189 & 3,73904 & Icels & 0,028 & 36,37704 \\
\hline Benin & 0,044 &, 52931 & Indi & 0,02 & 0,56430 \\
\hline Bhute & 0,0267 & 0,68173 & Isra & 0,0 & 16,48128 \\
\hline Bolivia & 0,057 & 0,91778 & Italy & 0,022 & 25,47110 \\
\hline $\begin{array}{l}\text { Bosnia \& } \\
\text { Herzegovina }\end{array}$ & 0,039 & 1,81962 & Jordan & 0,054 & 1,8738 \\
\hline Botswana & 0,068 & 4,37173 & Kazakhstan & 0,05 & 2,07242 \\
\hline Brazil & 0,026 & 2,86386 & Ken & 0,06 & 0,45043 \\
\hline Bulgaria & 0,0435 & 2,54876 & Kyrgyzstan & 0,112 & 0,37985 \\
\hline Burkina Faso & 0,079 & 0,34535 & Latvia & 0,031 & 4,77064 \\
\hline Burundi & 0,063 & 0,08264 & Lebanon & 0,019 & 4,42330 \\
\hline Cambodia & 0,0247 & 0,30839 & Lithuania & 0,032 & 5,31551 \\
\hline Canada & 0,019 & 27,07944 & Luxembourg & 0,006 & 59,14322 \\
\hline Chile & 0,0377 & 4,59061 & Macau, China & 0,0166 & 0,00000 \\
\hline Colombia & 0,038 & 1,79342 & Macedonia & 0,0176 & 2,27744 \\
\hline $\begin{array}{l}\text { Commonwealth } \\
\text { of Dominica }\end{array}$ & 0,0489 & 3,62553 & Madagascar & 0,0312 & 0,3240 \\
\hline Costa Rica & 0,11 & 4,36626 & Malaysia & 0,024 & 4,18728 \\
\hline Côte d'Ivoire & 0,076 & 0,82946 & Mali & 0,056 & 0,37300 \\
\hline Croatia & 0,036 & 6,48194 & Malta & 0,0135 & 11,95117 \\
\hline Cyprus & 0,0202 & 17,10745 & Mauritius & 0,033 & 4,28858 \\
\hline Czech Republic & 0,023 & 8,86330 & Mexico & 0,0424 & 6,24762 \\
\hline Ecuador & 0,035 & 2,09110 & Moldova & 0,094 & 0,46744 \\
\hline Egypt & 0,013 & 1,22008 & Morocco & 0,037 & 1,45210 \\
\hline El Salvador & 0,0496 & 2,28682 & Netherlands & 0,0141 & 31,53177 \\
\hline Estonia & 0,034 & 6,71254 & New Zealand & 0,0205 & 19,85657 \\
\hline Fiji & 0,057 & 2,68574 & Nicaragua & 0,057 & 0,75460 \\
\hline Finland & 0,0266 & 31,05830 & Niger & 0,086 & 0,23222 \\
\hline Germany & 0,0112 & 29,11475 & Nigeria & 0,0606 & 0,42226 \\
\hline Ghana & 0,143 & 0,36887 & Norway & 0,0193 & 48,41162 \\
\hline
\end{tabular}




\begin{tabular}{lrr|lrr} 
Land & NIM & PKE & Land & NIM & \multicolumn{1}{c}{ PKE } \\
\hline Oman & 0,038 & 8,34925 & Slovenia & 0,036 & 13,90920 \\
Pakistan & 0,0339 & 0,55477 & South Africa & 0,029 & 3,60984 \\
Panama & 0,033 & 4,31029 & South Korea & 0,0264 & 12,69260 \\
Papua New & 0,015 & 0,62247 & Spain & 0,0244 & 20,40446 \\
Guinea & 0,0893 & 1,06853 & Suriname & 0,063 & 2,32944 \\
Paraguay & 0,0505 & 2,23136 & Swaziland & 0,05925 & 1,72193 \\
Peru & 0,0266 & 0,97334 & Sweden & 0,011 & 33,67638 \\
Philippines & 0,0349 & 5,48620 & Switzerland & 0,0102 & 43,55350 \\
Poland & 0,021 & 14,16115 & Taiwan & 0,0157 & 12,67711 \\
Portugal & 0,026 & 2,61916 & Tajikistan & 0,0131 & 0,24416 \\
Romania & 0,019 & 2,99889 & Thailand & 0,0185 & 2,30517 \\
Russia & 0,057 & 0,20057 & Togo & 0,09 & 0,36181 \\
Rwanda & 0,052 & 7,90139 & Tonga & 0,073 & 1,65992 \\
Saint Kitts \& & & & Trinidad \& & 0,0341 & 8,00744 \\
Nevis & 0,058 & 4,31975 & Tobago & & \\
Saint Lucia & & & Tunisia & 0,022 & 2,53025 \\
Saint Vincent \& & 0,0346 & 3,44707 & & & \\
The Grenadines & & & & & \\
Samoa & 0,017 & 1,78480 & Turkey & 0,1119 & 3,39936 \\
(Western) & 0,028 & 9,43619 & Turkmenistan & 0,036 & 1,20025 \\
Saudi Arabia & 0,068 & 0,63327 & Ukraine & 0,065 & 1,03675 \\
Senegal & 0,019 & 2,53999 & United States & 0,0338 & 37,64846 \\
Serbia \& & 0,026 & 8,40383 & Uruguay & 0,0163 & 3,31071 \\
Montenegro & 0,014 & 21,73392 & Vanuatu & 0,008 & 1,31341 \\
Seychelles & 0,023 & 6,06549 & Venezuela & 0,079 & 3,24982 \\
Singapore & & & & & \\
Slovakia & & & &
\end{tabular}


A4: Schätzergebnisse - Economic Freedom Indicator, Teil 2

\begin{tabular}{|c|c|c|c|c|}
\hline & $1998-2000$ & Variante (1) & Variante (2) & Variante (3) \\
\hline GDPpc & $\begin{array}{r}-0.0002205 \\
(-0.73)\end{array}$ & $\begin{array}{r}-0.002623 \\
(-0.47)\end{array}$ & & \\
\hline $\mathrm{d} 34$ & $\begin{array}{r}0.0461679 \\
(1.13) \\
\end{array}$ & $\begin{array}{r}0.0461348 \\
(1.19) \\
\end{array}$ & $\begin{array}{r}0.046927 \\
(1.23) \\
\end{array}$ & \\
\hline $\begin{array}{l}\text { Economic } \\
\text { Freedom }\end{array}$ & $\begin{array}{r}0.0057431 \\
(0.47)\end{array}$ & $\begin{array}{r}0.0101707 \\
(1.49)\end{array}$ & $\begin{array}{r}* 0.113623 \\
(1.82)\end{array}$ & $\begin{array}{r}0.0083052 \\
(1.48)\end{array}$ \\
\hline Monetary & $\begin{array}{r}* * * \\
0.0101373\end{array}$ & $* * * 0.010222$ & $* * * 0.0105668$ & $* * * 0.0116993$ \\
\hline Policy & (4.24) & (4.74) & (5.27) & (6.20) \\
\hline Wages \& & $\begin{array}{r}* * \\
0.0095528\end{array}$ & ** -0.0087702 & $* *-0.0087996$ & $*-0.006375$ \\
\hline Prices & $(-2.24)$ & $(-2.18)$ & $(-2.22)$ & $(-1.71)$ \\
\hline $\begin{array}{l}\text { Foreign } \\
\text { Investment }\end{array}$ & $\begin{array}{r}0.0020355 \\
(0.51) \\
\end{array}$ & & & \\
\hline Banking & $\begin{array}{r}0.0025479 \\
(0.79) \\
\end{array}$ & & & \\
\hline $\begin{array}{l}\text { Property } \\
\text { Rights }\end{array}$ & $\begin{array}{r}0.0026558 \\
(0.58) \\
\end{array}$ & & & \\
\hline $\begin{array}{l}\text { Informal } \\
\text { Markets }\end{array}$ & $\begin{array}{r}-0.0018047 \\
(-0.59)\end{array}$ & & & \\
\hline Cons & $\begin{array}{r}0.0131946 \\
(0.78) \\
\end{array}$ & $\begin{array}{r}0.009682 \\
(0.61) \\
\end{array}$ & $\begin{array}{r}0.0041646 \\
(0.39) \\
\end{array}$ & $\begin{array}{r}0.0099491 \\
(1.16) \\
\end{array}$ \\
\hline $\begin{array}{l}\text { Countries } \\
\mathrm{R}^{2} \\
\text { Adj. } \mathrm{R}^{2} \\
\text { F-Wert }\end{array}$ & $\begin{array}{r}62 \\
0.6701 \\
0.6130 \\
F(9,52)= \\
11.74\end{array}$ & $\begin{array}{r}62 \\
0.6609 \\
0.6307 \\
F(5,56)= \\
21.83 \\
\end{array}$ & $\begin{array}{r}63 \\
0.6638 \\
0.6406 \\
F(4,58)=28.63\end{array}$ & $\begin{array}{r}69 \\
0.6562 \\
0.6403 \\
F(3,65)= \\
41.35 \\
\end{array}$ \\
\hline
\end{tabular}




\section{A5: Fragebogen der Weltbank-Umfrage ${ }^{1}$, Periode 1998 - 2000}

\section{Entry into Banking}

1.1 What body/agency grants commercial banking licenses?

1.2 How many commercial banks are there at present?

1.3 What are the minimum capital entry requirements (in U.S. \$ and/or domestic currency)?

1.4 Is it legally required that applicants submit information on the source of funds to be used as capital?

1.5 Are the sources of funds to be used as capital verified by the regulatory/supervisory authorities?

1.5.1 Are law enforcement authorities consulted in this process?

1.6 Can the initial disbursement or subsequent injections of capital be done with assets other than cash or government securities?

1.7 Can initial disbursement of capital be done with borrowed funds?

1.8 Which of the following are legally required to be submitted before issuance of the banking license?

1.8.1 Draft by-laws?

1.8.2 Intended organization chart?

1.8.3 Financial projections for first three years?

1.8.4 Financial information on main potential shareholders?

1.8.5 Background/experience of future directors?

1.8.6 Background/experience of future managers?

1.8.7 Sources of funds to be disbursed in the capitalization of new bank?

1.8.8 Market differentiation intended for the new bank?

1.9 In the past five years, how many applications for commercial banking licenses have been received from domestic entities?

1.9.1 How many of those applications have been denied?

1.10 In the past five years, how many applications for commercial banking licenses have been received from foreign entities?

1.10.1 How many of those applications have been denied?

1.11 What were the primary reasons for denial of the applications in 1.9.1 and 1.10 .1 ?

1.11.1 Capital amount or quality?

1.11.2 Banking skills?

1.11.3 Reputation?

1.11.4 Incomplete application?

Vgl. Barth, Caprio und Levine (2000). 


\section{Ownership}

2.1 Is there a maximum percentage of bank capital that can be owned by a single owner?

2.1.1 If yes, what is the percentage?

2.2 Can related parties own capital in a bank?

2.2.1 If yes, what are the maximum percentages associated with the total ownership by a related party group (e.g., family, business associates, etc.)?

2.3 What is the level of regulatory restrictiveness of ownership by nonfinancial firms of banks?

Unrestricted - A nonfinancial firm may own 100 percent of the equity in a bank.

Permitted - Unrestricted with prior authorization or approval.

Restricted - Limits are placed on ownership, such as a maximum percentage of a bank's capital or shares.

Prohibited - No equity investment in a bank.

2.4 What fraction of capital in the largest 10 banks is owned by commercial/industrial and/or financial conglomerates?

2.5 Can non-bank financial firms (e.g. insurance companies, finance companies, etc.) own commercial banks?

2.5.1 What are the limits?

2.6 Of deposit-taking institutions in your country, what fraction of deposits is held by the five (5) largest banks?

\section{Capital}

3.1 What is the minimum capital-asset ratio requirement?

3.1.1 Is this ratio risk weighted in line with the Basle guidelines?

3.2 Does the minimum ratio vary as a function of an individual bank's credit risk?

3.3 Does the minimum ratio vary as a function of market risk?

3.4 What is the actual risk-adjusted capital ratio in banks today (latest available data)?

3.5 Is subordinated debt allowable (required) as part of capital?

3.6 What fraction of revaluation gains is allowed as part of capital?

3.7 What fraction of the banking system's assets is in banks that are $50 \%$ or more government owned?

3.8 What fraction of the banking system's assets is in banks that are $50 \%$ or more foreign owned?

3.9 Before minimum capital adequacy is determined, which of the following are deducted from the book value of capital?

3.9.1 Market value of loan losses not realized in accounting books?

3.9.2 Unrealized losses in securities portfolios?

3.9.3 Unrealized foreign exchange losses? 


\section{Activities}

4.1 What is the level of regulatory restrictiveness for bank participation in securities activities ( the ability of banks to engage in the business of securities underwriting, brokering, dealing, and all aspects of the mutual fund industry) ?

Unrestricted - A full range of activities in the given category can be conducted directly in the bank.

Permitted - A full range of activities can be conducted, but all or some must be conducted in subsidiaries.

Restricted - Less than a full range of activities can be conducted in the bank or subsidiaries.

Prohibited - The activity cannot be conducted in either the bank or subsidiaries.

4.2 What is the level of regulatory restrictiveness for bank participation in insurance activities (the ability of banks to engage in insurance underwriting and selling)?

Unrestricted - A full range of activities in the given category can be conducted directly in the bank.

Permitted - A full range of activities can be conducted, but all or some must be conducted in subsidiaries.

Restricted - Less than a full range of activities can be conducted in the bank or subsidiaries.

Prohibited - The activity cannot be conducted in either the bank or subsidiaries.

4.3 What is the level of regulatory restrictiveness for bank participation in real estate activities (the ability of banks to engage in real estate investment, development, and management)?

Unrestricted - A full range of activities in the given category can be conducted directly in the bank.

Permitted - A full range of activities can be conducted, but all or some must be conducted in subsidiaries.

Restricted - Less than a full range of activities can be conducted in the bank or subsidiaries.

Prohibited - The activity cannot be conducted in either the bank or subsidiaries

4.4 What is the level of regulatory restrictiveness for bank ownership of nonfinancial firms?

Unrestricted - A full range of activities in the given category can be conducted directly in the bank.

Permitted - A full range of activities can be conducted, but all or some must be conducted in subsidiaries.

Restricted - Less than a full range of activities can be conducted in the bank or subsidiaries. 
Prohibited - The activity cannot be conducted in either the bank or subsidiaries.

\section{External Auditing Requirements}

5.1 Is an external audit a compulsory obligation for banks?

5.2 Are specific requirements for the extent or nature of the audit spelled out?

5.3 Are auditors licensed or certified?

5.4 Do supervisors get a copy of the auditor's report?

5.5 Does the supervisory agency have the right to meet with external auditors to discuss their report without the approval of the bank?

5.6 Are auditors required by law to communicate directly to the supervisory agency any presumed involvement of bank directors or senior managers in illicit activities, fraud, or insider abuse?

5.7 Can supervisors take legal action against external auditors for negligence?

5.8 Has action been taken against an auditor in the last 5 years?

\section{Internal Management/Organizational requirements}

6.1 Can the supervisory authority force a bank to change its internal organizational structure?

6.2 Has this power been utilized in the last 5 years?

\section{Liquidity \& Diversification Requirements}

7.1 Are there explicit, verifiable, and quantifiable guidelines regarding asset diversification?

7.2 Are banks prohibited from making loans abroad?

7.3 What are the requirements for bank in terms of liquidity reserves or any reserves whatsoever on deposits at the Central Bank?

7.4 What interest, if any, is paid on these reserves?

7.5 What is the minimum reserve requirement (\%)?

7.6 How is the reserve requirement remunerated?

7.7 What domestic and foreign assets satisfy these liquidity reserve or any other reserve requirements?

\section{Depositor (Savings) Protection Schemes}

8.1 Is there an explicit deposit insurance protection system? If yes:

8.1.1 Is it funded by (check one) : the government, the banks, or both ?

8.1.2 What is the ratio of accumulated funds to total bank assets?

8.1.3 What is the deposit insurance limit per account?

8.1.4 Is there a limit per person?

8.1.4.1 If yes, what is that limit (in domestic currency)?

8.1.5 Does the deposit insurance authority make the decision to intervene a bank?

8.1.6 If no, who does? 
8.1.7 If yes, does the deposit insurance authority have the legal power to deal with (intervene/takeover) a troubled (though perhaps still solvent) bank to reduce the ultimate burden on the deposit insurance fund?

8.2 As a share of total assets, what is the value of large denominated debt liabilities of banks-subordinated debt, bonds, etc.-that are definitely not covered by any explicit or implicit savings protection scheme?

8.3 As part of failure resolution, how many banks closed or merged in the last 5 years?

8.4 Were depositors wholly compensated (to the extent of legal protection) the last time a bank failed?

8.4.1 On average, how long does it take to pay depositors in full?

8.4.2 What was the longest that depositors had to wait in the last 5 years?

8.5 Were any deposits not explicitly covered by deposit insurance at the time of the failure compensated when the bank failed (excluding funds later paid out in liquidation procedures)?

8.6 Can the deposit insurance agency/fund take legal action against bank directors or other bank officials?

8.7 Has the deposit insurance agency/fund ever taken legal action against bank directors or other bank officials?

\section{Provisioning Requirements}

9.1 Is there a formal definition of a "non-performing loan" ?

9.1.1 If yes, what is it? Is the number of days in arrears the only or principal basis of asset classification and provisioning?

9.2 Classification of loans in arrears based on their quality: after how many days is a loan in arrears classified as:

9.2.1 Sub-standard?

9.2.2 Doubtful?

9.2.3 Loss?

9.3 What are the minimum required provision as loans become:

9.2.1 Sub-standard?

9.2.2 Doubtful?

9.2.3 Loss?

9.4 If you do not have a loan classification system based on sub-standard, doubtful and loss loans, please describe the type of classification system you do have.

9.5 What is the ratio of non-performing loans to total assets (latest available)?

9.6 If a customer has multiple loans and one loan is classified as non-performing, are the other loans automatically classified as non-performing?

\section{Accounting/Information Disclosure Requirements}

10.1 Does accrued, though unpaid, interest/principal enter the income statement while the loan is still performing? 
10.1.1 Does accrued, though unpaid, interest/principal enter the income statement while the loan is still non-performing?

10.2 After how many days in arrears must interest income accrual cease?

10.3 Are financial institutions required to produce consolidated accounts covering all bank and any non-bank financial subsidiaries?

10.4 Are off-balance sheet items disclosed to supervisors?

10.4.1 Are off-balance sheet items disclosed to the public?

10.5 Must banks disclose their risk management procedures to the public?

10.6 Are bank directors legally liable if information disclosed is erroneous or misleading?

10.6.1 Have penalties been enforced?

10.7 Do regulations require credit ratings for commercial banks?

10.7.1 What percentage of the top ten banks are rated by international credit rating agencies (e.g. Moody's, Standard and Poor)?

10.7.2 What percentage of the top ten banks are rated by domestic credit rating agencies?

10.7.3 Which bank activities are rated?

10.7.3.1 Bonds?

10.7.3.2 Commercial paper?

10.7.3.3 Other activity (e.g., bank certificates of deposit, pension and mutual funds, insurance companies, financial guarantees, etc.)?

\section{Discipline/Problem Institutions/Exit}

11.1 Are there any mechanisms of cease and desist-type orders, whose infraction leads to the automatic imposition of civil and penal sanctions on the banks directors and managers?

11.2 Can the supervisory agency order the bank's directors or management to constitute provisions to cover actual or potential losses?

11.3 Can the supervisory agency suspend the directors' decision to distribute:

11.3.1 Dividends?

11.3.2 Bonuses?

11.3.3 Management fees?

11.4 Have any such actions been taken in the last 5 years?

11.5 Which laws address bank insolvency?

11.6 Can the supervisory agency legally declare-such that this declaration supersedes the rights of bank shareholders-that a bank is insolvent?

11.7 Does the Banking Law give authority to the supervisory agency to intervenethat is, suspend some or all ownership rights-a problem bank?

11.8 Does the Law establish pre-determined levels of solvency deterioration which forces automatic actions (like intervention)?

11.9 Regarding bank restructuring and reorganization, can the supervisory agency or any other government agency do the following:

11.9.1 Supersede shareholder rights? 
11.9.2 Remove and replace management?

11.9.3 Remove and replace directors?

11.9.4 Forbear certain prudential regulations?

11.9.5 Insure liabilities beyond any explicit deposit insurance scheme?

11.10 How many banks have been closed in the last five years?

11.10.1 What percentage of total bank assets did those banks account for?

\section{Supervision}

12.1 What body/agency supervises banks?

12.1.1 Is there more than one supervisory body?

12.2 To whom are the supervisory bodies responsible or accountable?

12.2.1 How is the head of the supervisory agency (and other directors) appointed?

12.2.2 How is the head of the supervisory agency (and other directors) removed?

12.3 Are there important differences between what the supervisory agency is expected to do and what is mandated by law?

12.4 How many professional bank supervisors are there in total?

12.4.1 How many professional bank supervisors are there per institution?

12.5 How many onsite examinations per bank were performed in the last five years?

12.6 What is the total budget for supervision in local currency (in 1997 or 1998; please specify which)?

12.7 How frequently are onsite inspections conducted in large and medium size banks (annually equals 1 and every two years equals 2 )?

12.8 What is the average tenure of current supervisors (i.e., what is the average number of years current supervisors have been supervisors)?

12.9 How often are bank supervisors employed by the banking industry once they quit their service as bank supervisors? Never, Rarely, Occasionally, or Frequently?

12.10 If an infraction of any prudential regulation is found by a supervisor, must it be reported?

12.11 Are there mandatory actions in these cases?

12.12 Who authorizes exceptions to such actions?

12.13 How many exceptions were granted last year?

12.14 Are supervisors legally liable for their actions (e.g., if a supervisor takes actions against a bank can he/she be sued)? 


\section{A6: Fragebogen der Weltbank-Umfrage, Periode 2001 - 2003}

\section{Entry into Banking}

1.1 What body/agency grants commercial banking licenses?

1.1.1 Is there more than one body/agency that grants licenses to banks?

1.1.2 Is more than one license required (e.g., one for each banking activity, such as commercial banking, securities operations, insurance, etc.)?

1.2 How many commercial banks were there at year-end 2001?

1.2.1 What are the total assets of all commercial banks at year-end 2001?

1.2.2 What are the total deposits of all commercial banks at year-end 2001?

1.3 What are the minimum capital entry requirements (in U.S. $\$$ and/or domestic currency)?

1.3.1 Is this minimum capital entry requirement the same for a foreign branch and subsidiary?

1.4 Is it legally required that applicants submit information on the source of funds to be used as capital?

1.5 Are the sources of funds to be used as capital verified by the regulatory/supervisory authorities?

1.6 Can the initial disbursement or subsequent injections of capital be done with assets other than cash or government securities?

1.7 Can initial disbursement of capital be done with borrowed funds?

1.8 Which of the following are legally required to be submitted before issuance of the banking license?

1.8.1 Draft by-laws?

1.8.2 Intended organization chart?

1.8.3 Financial projections for first three years?

1.8.4 Financial information on main potential shareholders?

1.8.5 Background/experience of future directors?

1.8.6 Background/experience of future managers?

1.8.7 Sources of funds to be disbursed in the capitalization of new bank?

1.8.8 Market differentiation intended for the new bank?

1.9 In the past five years, how many applications for commercial banking licenses have been received from domestic entities?

1.9.1 How many of those applications have been denied?

1.10 In the past five years, how many applications for commercial banking licenses have been received from foreign entities? And how many have been denied?

1.10.1 Number of applications from foreign entities to enter through the acquisition of domestic bank?

1.10.2 Number of applications from foreign entities to enter through new, capitalized subsidiary?

1.10.3 Number of applications from foreign entities to enter through opening a branch? 
1.10.4 Number of applications from foreign entities to enter through some other means?

1.11 What were the primary reasons for denial of the applications in 1.9.1 and

1.10 .1 ?

1.11.1 Capital amount or quality?

1.11.2 Banking skills?

1.11.3 Reputation?

1.11.4 Incomplete application?

1.11.5 Other reasons(s). Please list.

1.12 Are foreign entities prohibited from entering through:

1.12.1 Acquisition?

1.12.2 Subsidiary?

1.12.3 Branch?

\section{Ownership}

2.1 Is there a maximum percentage of bank capital that can be owned by a single owner?

2.1.1 If yes, what is the percentage?

2.2 Can related parties own capital in a bank?

2.2.1 If yes, what are the maximum percentages associated with the total ownership by a related party group (e.g., family, business associates, etc.)?

2.2.2 Are there penalties for violating this rule?

2.3 What is the level of regulatory restrictiveness for nonfinancial firms ownership of bank:

Unrestricted - A nonfinancial firm may own 100 percent of the equity in a bank?

Permitted - Unrestricted with prior authorization or approval?

Restricted - Limits are placed on ownership, such as a maximum percentage of a bank's capital or shares?

Prohibited - No equity investment in a -bank?

2.4 What fraction of capital in the largest 10 banks is owned by commercial/industrial and/or financial conglomerates? If there are fewer than 10 banks, use that number in your answer.

2.5 What is the level of regulatory restrictiveness for non-bank financial firms (e.g., insurance companies, finance companies, etc.) ownership of commercial banks:

Unrestricted - A non-bank financial firm may own 100 percent of the equity in a bank?

Permitted - Unrestricted with prior authorization or approval?

Restricted - Limits are placed on ownership, such as a maximum percentage of a bank's capital or shares?

Prohibited - No equity investment in a bank? 
2.6 Of commercial banks in your country, what fraction of:

2.6.1 deposits is held by the five (5) largest banks at year-end 2001?

2.6.2 assets is held by the five (5) largest banks at year-end 2001?

2.7 Of all deposit-taking institutions in your country, what fraction of their assets is held by just commercial banks?

\section{Capital}

3.1 What is the minimum capital-asset ratio requirement?

3.1.1 Is this ratio risk weighted in line with the Basle guidelines?

3.2 Does the minimum ratio vary as a function of an individual bank's credit risk?

3.3 Does the minimum ratio vary as a function of market risk?

3.4 What is the actual risk-adjusted capital ratio in banks as of year-end 2001, using the 1988 Basle Accord definitions?

3.4.1 What is the actual equity capital ratio (i.e., not risk-adjusted) of banks as of year-end 2001 ?

3.5 Is subordinated debt allowable as part of capital?

3.6 Is subordinated debt required as part of capital?

3.7 What fraction of revaluation gains is allowed as part of capital?

3.8 What fraction of the banking system's assets is in banks that are:

3.8.150\% or more government owned as of year-end 2001 ?

3.8.2 $50 \%$ or more foreign owned as of year-end 2001 ?

3.9 Before minimum capital adequacy is determined, which of the following are deducted from the book value of capital:

3.9.1 Market value of loan losses not realized in accounting books?

3.9.2 Unrealized losses in securities portfolios?

3.9.3 Unrealized foreign exchange losses?

3.10 Are accounting practices for banks in accordance with International Accounting Standards (IAS)?

3.11 Are accounting practices for banks in accordance with U.S. Generally Accepted Accounting Standards (GAAS)?

\section{Activities}

4.1 What is the level of regulatory restrictiveness for bank participation in securities activities (the ability of banks to engage in the business of securities underwriting, brokering, dealing, and all aspects of the mutual fund industry):

Unrestricted - A full range of activities in the given category can be conducted directly in the bank?

Permitted - A full range of activities can be conducted, but all or some must be conducted in subsidiaries?

Restricted - Less than a full range of activities can be conducted in the bank or subsidiaries?

Prohibited - The activity cannot be conducted in either the bank or subsidiaries? 
4.2 What is the level of regulatory restrictiveness for bank participation in insurance activities (the ability of banks to engage in insurance underwriting and selling):

Unrestricted - A full range of activities in the given category can be conducted directly in the bank?

Permitted - A full range of activities can be conducted, but all or some must be conducted in subsidiaries?

Restricted - Less than a full range of activities can be conducted in the bank or subsidiaries?

Prohibited - The activity cannot be conducted in either the bank or subsidiaries?

4.3 What is the level of regulatory restrictiveness for bank participation in real estate activities (the ability of banks to engage in real estate investment, development, and management):

Unrestricted - A full range of activities in the given category can be conducted directly in the bank?

Permitted - A full range of activities can be conducted, but all or some must be conducted in subsidiaries?

Restricted - Less than a full range of activities can be conducted in the bank or subsidiaries?

Prohibited - The activity cannot be conducted in either the bank or subsidiaries?

4.4 What is the level of regulatory restrictiveness for bank ownership of nonfinancial firms:

Unrestricted - A bank may own 100 percent of the equity in any nonfinancial firm?

Permitted - A bank may own 100 percent of the equity in a nonfinancial firm, but ownership is limited based on a bank's equity capital?

Restricted - A bank can only acquire less than 100 percent of the equity in a nonfinancial firm?

Prohibited - A bank may not acquire any equity investment in a nonfinancial firm?

\section{External Auditing Requirements}

5.1 Is an external audit a compulsory obligation for banks?

5.2 Are specific requirements for the extent or nature of the audit spelled out?

5.3 Are auditors licensed or certified?

5.4 Do supervisors get a copy of the auditor's report?

5.5 Does the supervisory agency have the right to meet with external auditors to discuss their report without the approval of the bank? 
5.6 Are auditors required by law to communicate directly to the supervisory agency any presumed involvement of bank directors or senior managers in illicit activities, fraud, or insider abuse?

5.7 Can supervisors take legal action against external auditors for negligence?

5.8 Has action been taken against an auditor in the last 5 years?

\section{Internal Management/Organizational requirements}

6.1 Can the supervisory authority force a bank to change its internal organizational structure?

6.2 Has this power been utilized in the last 5 years?

\section{Liquidity \& Diversification Requirements}

7.1 Are there explicit, verifiable, and quantifiable guidelines regarding asset diversification (for example, are banks required to have some minimum diversification of loans among sectors, or are their sectoral concentration limits)?

7.2 Are banks prohibited from making loans abroad?

7.3 Are banks required to hold either liquidity reserves or any deposits at the Central Bank?

7.3.1 If so, what are these requirements?

7.4 Do these reserves earn any interest?

7.4.1 What interest is paid on these reserves?

7.5 Are banks allowed to hold reserves in foreign denominated currencies or other foreign denominated instruments? If yes, please state the ratio

7.6 Are banks required to hold reserves in foreign denominated currencies or other foreign denominated instruments? If yes, please state the ratio

7.7 What percent of the commercial banking system's assets is foreign-currency denominated?

7.8 What percent of the commercial banking system's liabilities is foreigncurrency denominated?

7.9 What percent of the commercial banking system's assets is in central government bonds?

7.10 What percent of the commercial banking system's assets is funded with deposits?

7.10.1 What percent of the commercial banking system's assets is funded with insured deposits?

\section{Depositor (Savings) Protection Schemes}

8.1 Is there an explicit deposit insurance protection system?

If no, you may skip to question 8.2. If yes:

8.1.1 Is it funded by (check one) : the government, the banks, or both ?

8.1.2 Are premia collected regularly (ex ante)

only when there is a need (ex post)

or both? 
8.1.3 Do deposit insurance fees charged to banks vary based on some assessment of risk?

8.1.4 If pre-funded, what is the ratio of accumulated funds to total bank assets?

8.1.5 What is the deposit insurance limit per account (in US\$ and local currency)?

8.1.5.1 US\$:

8.1.5.2 Domestic currency:

8.1.6 Is there a limit per person?

8.1.6.1 If yes, what is that limit (in domestic currency)?

8.1.7 Is there formal co-insurance, that is, are depositors only insured for some percentage of their deposits, either absolutely or above some floor and/or up to some limit?

8.1.8 Does the deposit insurance scheme also cover foreign currency deposits?

8.1.9 Are interbank deposits covered?

8.1.10 Does the deposit insurance authority make the decision to intervene a bank?

8.1.10.1 If no, who does?

8.1.11 Does the deposit insurance authority have the legal power to cancel or revoke deposit insurance for any participating bank?

8.2 As a share of total assets, what is the value of large denominated debt liabilities of banks-subordinated debt, bonds, etc.-that are definitely not covered by any explicit or implicit savings protection scheme?

8.3 As part of failure resolution, how many banks closed or merged in the last 5 years?

8.4 Were depositors wholly compensated (to the extent of legal protection) the last time a bank failed?

8.4.1 On average, how long does it take to pay depositors in full?

8.4.2 What was the longest that depositors had to wait in the last 5 years?

8.5 Were any deposits not explicitly covered by deposit insurance at the time of the failure compensated when the bank failed (excluding funds later paid out in liquidation procedures)?

8.6 Can the deposit insurance agency/fund take legal action against bank directors or other bank officials?

8.7 Has the deposit insurance agency/fund ever taken legal action against bank directors or other bank officials?

8.8 Are non-residents treated differently than residents with respect to deposit insurance scheme coverage?

8.9 Who manages the insurance fund? Is it managed:

a. solely by the private sector?

b. jointly by private-public officials?

c. solely by public sector?

8.10. Is participation in the deposit insurance system compulsory for all banks? 


\section{Provisioning Requirements}

9.1 Is there a formal definition of a "non-performing loan"?

9.1.1 The primary system for loan classification is based on (PLEASE PICK ONE):

(a) the number of days a loan is in arrears

(b) a forward looking estimate of the probability of default

(c) other

9.2 How many days is a loan in arrears classified as:

9.2.1 Sub-standard?

9.2.2 Doubtful?

9.2.3 Loss?

9.3 What is the minimum provisioning required as loans become:

9.3.1 Sub-standard?

9.3.2 Doubtful?

9.3.3 Loss?

9.4 What is the ratio of non-performing loans to total assets as of year-end 2001?

9.5 If a customer has multiple loans and one loan is classified as non-performing, are the other loans automatically classified as non-performing?

9.6 What is the aggregate net interest margin-to-asset ratio as of year-end 2001?

9.7 What is the aggregate overhead costs-to-asset ratio as of year-end 2001?

9.8 What is the tax deductibility of provisions:

9.8.1 Specific provisions can be deducted?

9.8.2 General provisions can be deducted?

9.8.3 Provisions cannot be deducted?

\section{Accounting/Information Disclosure Requirements}

10.1 Does accrued, though unpaid, interest/principal enter the income statement while the loan is still performing?

10.1.1 Does accrued, though unpaid, interest/principal enter the income statement while the loan is still non-performing?

10.2 After how many days in arrears must interest income accrual cease?

10.3 Are financial institutions required to produce consolidated accounts covering all bank and any non-bank financial subsidiaries?

10.4 Are off-balance sheet items disclosed to supervisors?

10.4.1 Are off-balance sheet items disclosed to the public?

10.5 Must banks disclose their risk management procedures to the public?

10.6 Are bank directors legally liable if information disclosed is erroneous or misleading?

10.6.1 What are the penalties, if applicable?

10.6.2 Have penalties been enforced?

10.7 Do regulations require credit ratings for commercial banks?

10.7.1 What percentage of the top ten banks are rated by international credit rating agencies (e.g. Moody's, Standard and Poor)? 
10.7.2 How many of the top ten banks are rated by domestic credit rating agencies?

10.7.3 Which bank activities are rated?

10.7.3.1 Bonds?

10.7.3.2 Commercial paper?

10.7.3.3 Other activity (e.g., bank certificates of deposit, pension and mutual funds, insurance companies, financial guarantees, etc.)?

\section{Discipline/Problem Institutions/Exit}

11.1 Are there any mechanisms of cease and desist-type orders, whose infraction leads to the automatic imposition of civil and penal sanctions on the banks directors and managers?

11.1.1 Are bank regulators/supervisors required to make public formal enforcement actions, which include cease-and desist orders and written agreements between a bank regulatory/supervisory body and a banking organization?

11.2 Can the supervisory agency order the bank's directors or management to constitute provisions to cover actual or potential losses?

11.3 Can the supervisory agency suspend the directors' decision to distribute:

11.3.1 Dividends?

11.3.2 Bonuses?

11.3.3 Management fees?

11.4 Have any such actions been taken in the last 5 years?

11.5 Which laws address bank insolvency?

11.6 Can the supervisory agency legally declare-such that this declaration supersedes the rights of bank shareholders-that a bank is insolvent?

11.7 Does the Banking Law give authority to the supervisory agency to intervenethat is, suspend some or all ownership rights-a problem bank?

11.8 Does the Law establish pre-determined levels of solvency deterioration which forces automatic actions (like intervention)?

11.9 Regarding bank restructuring and reorganization, can the supervisory agency or any other government agency do the following:

11.9.1 Supersede shareholder rights?

11.9.2 Remove and replace management?

11.9.3 Remove and replace directors?

11.9.4 Forbear certain prudential regulations?

11.9.5 Insure liabilities beyond any explicit deposit insurance scheme?

11.10.1 During the last five years, how many banks have been resolved in the following way, and what was the percentage of assets of the banking system accounted for by each:

a. Closure and liquidation?

b. Intervention and open bank assistance?

c. Transfer of assets and liabilities (incl. purchase and assumption) or merger and acquisition. Other (please specify)? 
11.10.3 How many months did each of these resolution techniques take on average, from the moment of intervention by the responsible authority to the moment of resolution?

11.11 Who is responsible for appointing and supervising a bank liquidator/ receiver?

11.12 Is court approval required for supervisory actions, such as superceding shareholder rights, removing and replacing management, removing and replacing director, or license revocation?

11.13 Is court order required to appoint a receiver/liquidator in the event of liquidation?

11.14 Can the bank shareholders appeal to the court against a decision of the bank supervisor?

\section{Supervision}

12.1 What body/agency supervises banks?

12.1.1 Is there more than one supervisory body?

12.2 To whom are the supervisory bodies responsible or accountable?

12.2.1 How is the head of the supervisory agency (and other directors) appointed?

12.2.2 Does the head of the supervisory agency (and other directors) have a fixed term? If yes, how long is the term?

12.2.3 The head of the supervisory agency can be removed by:

a: the decision of the head of government (e.g. President, Prime Minister)?

$\mathrm{b}$ : the decision of the Finance Minister or other cabinet level authority?

c: a simple majority of a legislative body (Parliament or Congress)?

d: a supermajority (e.g., $60 \%, 75 \%$ ) of a legislative body?

e: other?

12.3 Are there important differences between what the supervisory agency is expected to do and what is mandated by law?

12.4 How many professional bank supervisors are there in total?

12.5 How many onsite examinations per bank were performed in the last five years (annually, every two years, less frequently)?

12.6 What is the total budget for supervision in local currency or dollars (please specify) in 2002 ?

12.7 How frequently are onsite inspections conducted in large and medium size banks?

12.8 How many of the total bank supervisors have more than 10 years of experience in bank supervision?

12.8.1 What is the average tenure of current supervisors i.e. (what is the average number of years current supervisors have been supervisors)?

12.9 If an infraction of any prudential regulation is found by a supervisor, must it be reported?

12.9.1 Are there mandatory actions in these cases?

12.9.2 Who authorizes exceptions to such actions? 
12.9.3 How many exceptions were granted last year?

12.10 Are supervisors legally liable for their actions (e.g., if a supervisor takes actions against a bank can he/she be sued)? 


\section{Literaturverzeichnis}

Allen, $L .:$ The Determinants of Bank Interest Margins: A Note, Journal of Financial and Quantitative Analysis, Bd. 23, Nr. 2, Seattle, 1988.

Angbazo, L.: Commercial Bank Net Interest Margins, Default Risk, Interest Rate Risk, and Off-Balance Sheet Banking, Journal of Banking and Finance, Bd. 21, Nr. 6, 55-87, Amsterdam 1997.

Arrow, K.L.: Aspects of the Theory of Risk Bearing, (Johnsonin Saatie), Helsinki, 1964.

Australian Productivity Commission: Measures of Restrictions on Trade in Services Database, www.pc.gov.au/memoranda/servicesrestriction/index.html, 2001.

Baldwin, R.E.: Nontariff Distortions of International Trade, Washington, The Brookings Institutions, (zitiert in Chambers/Pick 1994, 48), 1970.

Baltensperger, E.: The Economic Theory of Banking Regulation in: Furubotn, E.G.; Richter, R. (Hrsg.), The Economics and Law of Banking Regulation, (1989/90), Center for the Study of the New Institutional Economics, Occasional Papers, Bd. 2, Saarbrücken, 1-22, 1989/90.

Barth, J.R.; Caprio, G.; Levine, R.: Bank Regulation and Supervision: What Works Best?, NBER Working Paper 9323, Cambridge (Mass.), 2002.

Barth, J.R.; Caprio, G.; Levine, R.: The Regulation and Supervision of Banks around the World - A New Database, World Bank, Policy Research Working Paper Nr. 2588, Washington D.C., 2001.

Beach, W.W.; Miles, M.A.: Explaining the Factors of the Index of Economic Freedom, Chapter 5, www. heritage.org, 2006.

Bhattacharya,S.; Thakor, A.V.: Contemporary Banking Theory, Journal of Financial Intermediation, Bd. 3, Nr. 1, 2-50, 1993.

Buch, C.M.; DeLong, G.: Determinants of Cross-Border Bank Mergers: Is Europe Different?, in: Herrmann, H.; Lipsey, R. (Hrsg.): Foreign Direct Investment in the Real and Financial Sector of Industrial Countries, Berlin u.a., 2003.

Buch, C.M.; Lipponer, A.: FDI versus cross-border financial services: The Globalisation of German Banks, Deutsche Bundesbank, Discussion Paper Series 1: Studies of the Economic Research Centre, Nr. 05/2004, Frankfurt a.M., 2004.

Chen, Z.; Schembri, L.: Measuring the Barriers to Trade in Services: Literature and Methodologies, in: Curtis, J.M.; Curiak, D. (Hrsg.): Trade Policy Research, 219-286, Ottawa, 2002. 
Claessens, S.: Competitive Implications of Cross-Border Banking, World Bank, Working Paper WPS 3854, Washington D.C., 2006.

Claessens, S.; Demirgüc-Kunt, A.; Huizinga, H.: How does Foreign Entry Affect the Domestic Banking Market? Worldbank Policy Research Working Paper, Nr. 1918, Washington D.C., 1998.

Claessens, S.; Glaessner, T.: Internationalisation of Financial Services in Asia, World Bank Discussion Paper, Nr. 1911, Washington D.C., 1998.

Cybo-Ottone, A.; Murgia, M.: Mergers and Shareholders Wealth in European Banking, Journal of Banking and Finance, Bd. 24, Nr. 6, Amsterdam, 2000.

Deardorff, A.V.; Stern, R.M.: Measurement of Non-Tariff Barriers, OECD Economic Department Working Paper No. 179, Paris, 1997.

Demirgüc-Kunt, A.; Laeven, L.; Levine, $R$.: The Impact of Bank Regulations, Concentrations, and Institutions on Bank Margins, World Bank, Policy Research Working Paper, Nr. 3030, Washington D.C., 2003.

Demirgüc-Kunt, A.; Laeven, L.; Levine, R.: Regulations, Market Structure, Institutions, and the Cost of Financial Intermediation, Journal of Money, Credit, and Banking, Bd. 36, Nr. 3, 593-622, Columbus, 2004.

Djordjevic, M.: Domestic Regulation and Free Trade in Services - A Balancing Act, Legal Issues of Economic Integration, Bd. 29, Nr. 3,The Hague, 2002.

Drakos, K.: The Dealership Model for Interest Margins: The Case of Greek Banking Industry, Journal of Emerging Market Finance, Bd. 1, Nr. 1, New Delhi, 2002.

Eilenberg, G.: Bankbetriebswirtschaftlehre: Grundlagen - Internationale Bankleistungen - Bank-Management, 3. Auflage, München u.a., 1987.

Ellingsen, T.; Wärneryd, K.: Foreign Direct Investment and the Political Economy of Protection, International Economic Review, Bd. 40., Nr. 2, 357-379, Mai 1990.

English, W.B.: Interest Rate Risk and Bank Net Interest Margins, BIS Quarterly Review, 67-82, Basel, Dezember 2002.

Feketekuty, G.: International Trade in Services. An Overview and Blueprint for Negotiations, Cambridge, Ma., 1988.

Fetzer, M.-U.: Ordnungspolitische Probleme einer Liberalisierung des internationalen Dienstleistungsverkehrs - Das Beispiel der Banken und Versicherungen in der EG, München, 1992.

Francois, J.F.; Eschenbach, F.: Financial Sector Competition, Service Trade, and Growth, Discussion Paper Nr. 2002-089.2, Tinbergen Institute, Amsterdam, 2002. 
Geigant, F.; Haslinger, F.; Sobotka, D.; Westphal, H.M.: Lexikon der

Volkswirtschaft , 7. Auflage, Landsberg, 2000.

Hackl, P.: Einführung in die Ökonometrie, München, 2005.

Hardin, A.; Holmes, L.: Services Trade and Foreign Direct Investment, Industry Commission Staff Research Paper, AGPS, Canberra, 1997.

Hillman, J.S.: Technical Barriers to Agricultural Trade, Westview Press, 1991.

Ho, T.; Saunders, A.: The Determinants of Bank Interest Margins: Theory and

Empirical Evidence, Journal of Financial and Quantitative Analysis, Bd. 16, Nr. 4, 581-600, Seattle, 1981.

Hoekman, B.: The Next Round of Service Negotiations: Identifying Priorities and Options, Federal Reserve Bank of St. Louis Review, Bd. 82, Juli/August, 31-47, St. Louis, 2000.

Hoekman, B.: Tentative First Steps. An Assessment of the Uruguay Round Agreement on Services, Annex 1, Policy Research Working Paper Nr. 1455, The World Bank, Washington D.C., 1995.

IMF / World Bank: Market Access for Developing Countries' Export, Prepared by the Staffs of the IMF and the World Bank, April 27, 2001.

McShane, R.W.; Sharpe, I.G.: A Time Series/Cross Section Analysis of the Determinants of Australian Trading Bank Loan/Deposit Interest Margins: 1962 - 1981, Journal of Banking and Finance, Bd. 9, 115-136, Amsterdam, 1985.

Kalirajan, K.; McGuire, G.; Nguyen-Hong, D.; Schuele, M.: The Price Impact of Restrictions on Banking Services, in: Findlay, C.; Warren, T. (Hrsg.): Impediments To Trade in Services - Measurement and Policy Implications, London, 2000.

Kannan, R; Narain, A.; Ghosh, S.: Determinants of Net Interest Margin under Regulatory Requirements - An Econometric Study, Economic and Political Weekly, January 27, 337-344, Mumbai (Bombay), 2001.

Krancke, J.: Liberalisierung des internationalen Dienstleistungshandels: Analyse des GATS und Perspektiven für die zukünftige Handelsliberalisierung, Kieler Arbeitspapiere Nr. 954, Kiel, 1999.

Krugman, P.R.; Obstfeld, M.: International Economics - Theory and Policy, 6. Aufl., Boston u.a., 2003.

Levine, R.: Foreign Banks, Financial Developments and Economic Growth, in: Barfield, C.E. (Hrsg.): International Financial Markets, 224-54, Washington D.C., 1996.

Hwang S.I.; Shin, I.; Yoo, J.: Korea's Liberalization of Financial Service Trade, in: Ito, T.; Krueger, A.O. (Hrsg.): Trade in Services in the Asia-Pacific 
Region, NBER-East Asia Seminar in Economics, Bd. 11, 283-314, Chicago and London, 2003.

Maennig, W.; Wilfling, B.: Außenwirtschaft - Theorie und Politik, München 1998.

Maleri, R.: Grundlagen der Dienstleistungsproduktion, 3. Auflage, Berlin u.a., 1994.

Matthes, J.: Neuer Protektionismus?: Perspektiven für eine weitere Liberalisierung des Welthandels, Köln, 2001.

Mattoo, A.; Wunsch, S.: Pre-empting Protectionism in Services: The WTO and Outsourcing, World Bank Policy Research Working Paper 3237, Washington D.C., 2004.

Mattoo, A.; Rathindran, R.; Subramanian, A.: Measuring Services Trade Liberalization and Its Impact on Trade Growth: An Illustration, World Bank Working Paper 2655, Washington D.C., 2001.

Mattoo, A.: Financial Services and the World Trade Organization - Liberalization Commitments of Developing and Transition Economies, World Bank Discussion Paper Nr. 2184, Washington D.C., 1998.

McGuire, G.: Australia's Restrictions on Trade in Financial Services, Productivity Commission Staff Research Paper, AusInfo, Canberra, 1998.

Mc Guire, G.; Schuele, M.: Restrictiveness of International Trade in Banking Services, in: Findlay, C.; Warren, T. (Hrsg): Impediments to Trade in Services: Measurement and Policy Implications, New York, 2001.

Minsky, H.P.: The financial instability hypothesis: A restatement., Thames Papers in Political Economy, Autumn 1978, reprinted in Arestis, P.; Skouras T. (Hrsg.): Post Keynesian Economic Theory, 24-55, Armonk, 1985.

Möschel, W.: International free trade in banking services, The Economics and Law of Banking Regulation, Saarbrücken, 67-88, 1990.

Mishkin, F.S.: The Economics of Money, Banking and Financial Markets, 6. Auflage, Boston u.a., 2001.

OECD: GATS - The Case for Open Service Markets, Paris, 2002.

OECD: Bank Profitability - Financial Statements of Banks, Paris 2002.

o.V.: Cross-Border Trade in Financial Services: Economics and Regulation, Financial Market Trends (OECD), Nr. 75, 23-60, Paris, 2000.

Poveda, E.C.; Droege, S.: International Trade in Financial Services: An Assessment of the GATS, Aussenwirtschaft, Bd. 52, Nr. 4, 603-635, Zürich, 1997. 
Pratt, J.W.: Risk Aversion in the Small and the Large, Econometrica Bd. 32, Jan.Apr., 122-136, Evanston, 1964.

Porter, M.E.:

The Competitive Advantage of Nations, London, 1990.

Rada, J.F.:

Information, Technology and Services, in: Giarini, O. (Hrsg.): The Emerging Service Industry, 127-171, Oxford u.a., 1987.

Reserve Bank of Australia:

International Comparisons of Bank Margins, Submission to the House of Representatives, Standing Committee on Banking, Finance and Public Administration, August 1994.

Reszat, B.: Regulierung der Banken in: Krakowski, M. (Hrsg.), Regulierungen in der Bundesrepublik Deutschland - Die Ausnahmebereiche des Gesetzes gegen Wettbewerbsbeschränkungen, Veröffentlichungen des HWWAInstituts für Wirtschaftsforschung, 347-422, Hamburg, 1988.

Robinson, S.; Wang, Z.; Martin, W.:Capturing the Implications of Services Trade Liberalization, Journal of the Economic System Research, Bd. 40, Nr. 1, 333, Abingdon, 2002.

Rosen, H.S.; Windisch, R.: Finanzwissenschaft I, München u.a., 1992.

Rück, H.R.G.: Dienstleistungen in der ökonomischen Theorie, Deutscher Universitäts-Verlag, Wiesbaden, 2000, zugleich Diss., Frankfurt a.M., 1999.

Rugman, A.M.: Multinationals and Trade in Services: A Transaction Cost Approach, in: Weltwirtschaftliches Archiv, Review of World Economics, Bd. 123, Nr. 4, 651-667, Hamburg, 1987.

Sampson, G.P.; Snape, R.H.: Identifying the Issues in Trade in Services, The World Economy, Bd. 8, 171-182, Oxford, 1985.

Sapir, A.: The General Agreement on Trade in Services - From 1994 to the Year 2000, Journal of World Trade, Bd. 33, Nr. 1, 51-66, London, 1999.

Sapir, A.: Kommentar zu J. Bhagwati „International Trade in Services and its Relevance for Economic Development, in: Garini, O. (Hrsg.), The Emerging Service Economy, 49-54, Oxford u.a., 1987.

Sapir, A.: Trade in Services: Policy Issues for the Eighties, The Columbia Journal of World Business, Bd. 17, New York, 1982.

Saunders, A.; Schumacher, L.: The Determinants of Bank Interest Rate Margins: An International Study, Journal of International Money and Finance, Bd. 19, Nr. 6, 813-832, Oxford u.a., 2000. 
Schnyder, M.: Die Hypothese finanzieller Instabilität von Hyman P. Minsky. Ein Versuch der theoretischen Abgrenzung und Erweiterung, Dissertation, Freiburg/Schweiz, 2002.

Schultz, S.: Protektionismus im Dienstleistungsverkehr, in: Giersch, H.(Hrsg.): Probleme und Perspektiven der weltwirtschaftlichen Entwicklung, Schriften des Vereins für Socialpolitik, Bd. 148, Berlin, 1985.

Smith, R.T.: Banking Competition and Macroeconomic Performance, Journal of Money, Credit and Banking, Bd. 30, Nr. 4, 1998.

Stern, R.M.; Hoekman, B.M.: Issues and Data Needs for GATT Negotiations on Services, The World Economy, Bd. 10, 39-60, Oxford, 1987.

Stibora, J.J.; Vaal, A. de: Services and Service Trade: A Theoretical Inquiry, Tinbergen Institute Research Series Nr. 95, Amsterdam, 1995.

Tschögel, A.E.: International Banking Centers, Geography and Foreign Banks, Financial Markets, Institutions and Instruments, Bd. 9, Nr. 1, 1-32, Malden (MA), 2003.

Walter, I.: Nontariff Protection Among Industrialized Countries: Some Preliminary Empirical Evidence, Economia Internazionale, Bd. 25, 335-54, Genf, 1972.

Walter, I.: Country Risk, Portfolio Decisions and Regulation in International Bank Lending, Journal of Banking an Finance, Bd. 5, Nr. 1, 77-92, Amsterdam, 1981.

Walter, I.: Barriers to Trade in Banking and Financial Services, Thames Essay Nr. 41, London, 1985.

Weck-Hannemann, H.: Politische Ökonomie des Protektionismus: eine institutionelle und empirische Analyse, Frankfurt a.M., 1992.

Weth, M.A.: The Pass-through from Market Interest Rates of Bank Lending Rates in Germany, Discussion Paper 11/02, Deutsche Bundesbank, Frankfurt a.M., 2002.

World Bank: World Development Indicator, Quick Query, http://ddpext.worldbank.org.

Zarruk, E.R.: Bank Spread with Uncertain Deposit Level and Risk Aversion, Journal of Banking and Finance, Bd. 13, 797-810, Amsterdam, 1989. 


\section{SCHRIFTEN ZUR WIRTSCHAFTSTHEORIE UND WIRTSCHAFTSPOLITIK}

Herausgegeben von Klaus Beckmann, Michael Berlemann, Rolf Hasse, Jörn Kruse, Franco Reither, Wolf Schäfer, Thomas Straubhaar, Klaus W. Zimmermann

Band 1 Lars Bünning: Die Konvergenzkriterien des Maastricht-Vertrages unter besonderer Berücksichtigung ihrer Konsistenz. 1997.

Band 2 Andreas Henning: Beveridge-Kurve, Lohnsetzung und Langzeitarbeitslosigkeit. Eine theoretische Untersuchung unter Berücksichtigung des Insider-Outsider-Ansatzes und der Entwertung des Humankapitals. 1997.

Band 3 Iris Henning: Die Reputation einer Zentralbank. Eine theoretische Untersuchung unter besonderer Berücksichtigung der Europäischen Zentralbank. 1997.

Band 4 Rüdiger Hermann: Ein gemeinsamer Markt für Elektrizität in Europa. Optionen einer Wettbewerbsordnung zwischen Anspruch und Wirklichkeit. 1997.

Band 5 Alexander Tiedtke: Japan und der Vorwurf des Trittbrettfahrerverhaltens in der US-amerikanisch-japanischen Allianz. 1997.

Band 6 Wolfgang Grimme: Ordnungspolitisches Konzept der Regionalpolitik. Darstellung der Defizite und des Reformbedarts der Regionalpolitik am Beispiel Mecklenburg-Vorpommerns. 1997.

Band 7 Christian Ricken: Determinanten der Effektivität der Umweltpolitik. Der nationale Politikstil im Spannungsfeld von Ökonomie, Politik und Kultur. 1997.

Band 8 Christian Schmidt: Real Convergence in the European Union. An Empirical Analysis. 1997.

Band 9 Silvia Marengo: Exchange Rate Policy for MERCOSUR: Lessons from the European Union. 1998.

Band 10 Jens Kleinemeyer: Standardisierung zwischen Kooperation und Wettbewerb. Eine spieltheoretische Betrachtung. 1998.

Band 11 Stefan M. Golder: Migration und Arbeitsmarkt. Eine empirische Analyse der Performance von Ausländem in der Schweiz. 1999.

Band 12 Stefan Kramer: Die Wirkung einer Internationalisierung des Yen auf die japanischen Finanzmärkłe, die japanische Geldpolitik und die Usancen der Fakturierung. 1999.

Band 13 Antje Marielle Gerhold: Wirtschaftliche Integration und Kooperation im asiatisch-pazifischen Raum. Die APEC. 1999.

Band 14 Tamim Achim Dawar. Deutsche Direktinvestitionen in Australien. Eine Evaluation der theoretischen Erklärungsansätze und der Standortattraktivität des Produktions- und Investitionsstandortes Australien. 1999.

Band 15 Hans-Markus Johannsen: Die ordnungspolitische Haltung Frankreichs im Prozeß der europäischen Einigung. 1999.

Band 16 Annette Schönherr. Vereinigungsbedingte Dimensionen regionaler Arbeitsmobilität. Wirkungen unter analytisch einfachen Bedingungen und potentielle individuelle Migrationsgewinne in Deutschland nach der Vereinigung. 1999.

Band 17 Henrik Müller. Wechselkurspolitik des Eurolandes. Konfliktstoff für die neue währungspolitische Ära. 1999.

Band 18 Lars H. Wengorz: Die Bedeutung von Unternehmertum und Eigentum für die Existenz von Unternehmen. Eine methodenkritische Analyse der Transformation des Wirtschaftssystems in Russland. 2000.

Band 19 Eckart Bauer. Konzeptionelle Grundfragen eines Kinderleistungsausgleichs im Rahmen einer umlagefinanzierten zwangsweisen Rentenversicherung. 2000.

Band 20 Hubertus Hille: Enlarging the European Union. A Computable General Equilibrium Assessment of Different Integration Scenarios of Central and Eastern Europe. 2001. 
Band 21 Tobias Just: Globalisierung und Ideologie. Eine Analyse der Existenz und Persistenz von Partisaneffekten bei zunehmender Internationalisienung der Märkte. 2001.

Band 22 Simone Claber: Großbritannien und die Europäische Integration unter besonderer Berücksichtigung ordnungspolitischer Aspekte. 2002.

Band 23 Silvia Rottenbiller: Essential Facilities als ordnungspolitisches Problem. 2002.

Band 24 Peggy Dreyhaupt von Speicher. Die Regionen Polens, Ungarns und der Tschechischen Republik vor dem EU-Beitritt. Interregionale Disparitäten, Bestimmungsfaktoren und Lösungsansätze. 2002.

Band 25 Gerhard Rösl: Seigniorage in der EWU. Eine Analyse der Notenbankgewinnentstehung und -verwendung des Eurosystems. 2002.

Band 26 Jörn Quitzau: Die Vergabe der Femsehrechte an der Fußball-Bundesliga. Wohlfahrtsökonomische, wettbewerbspolitische und sportökonomische Aspekte der Zentralvermarktung. 2003.

Band 27 Malgorzata Stankiewicz: Die polnische Telekommunikation vor dem EU-Beitritt. 2003.

Band 28 Sarah Schniewindt: Einführung von Wettbewerb auf der Letzten Meile. Eine ökonomische Analyse von Netzinfrastruktur und Wettbewerbspotential im Teilnehmeranschlussbereich. 2003.

Band 29 Jens Bruderhausen: Zahlungsbilanzkrisen bei begrenzter Devisenmarkteffizienz. Ein kapitalmarkttheoretischer Ansatz. 2004.

Band 30 Philip Jensch: Einkommensteuerreform oder Einkommensteuerersatz? Altemative Ansätze einer Reform der direkten Besteuerung unter besonderer Berücksichtigung steuerpraktischer Gesichtspunkte. 2004.

Band 31 Karsten J. Adamski: Mikroanalyse eines Grundrentenkonzeptes der Alterssicherung. Eine empirische Studie zur Wirkung einer Grundrente auf Basis des Sozioökonomischen Panels. 2004.

Band 32 Sven Schulze: Anreizwirkungen und Arbeitsmarkteffekte der Ausgestaltung einer Arbeitslosenversicherung. 2004.

Band 33 Alkis Henri Otto: Makroökonomische Effekte der Direktinvestitionen. 2005.

Band 34 Stefan Hardege: Informationstechnologische Entwicklung und der Schutz von Verfügungsrechten für Informationsgüter. Eine ökonomische Analyse zur Ausgestaltung des Urheberrechts. 2006.

Band 35 Christoph Kimmel: Vermögenspreisinflation als wirtschaftspolitische Herausforderung. 2008.

Band 36 Axel Czaya: Das Europäische Normungssystem aus der Perspektive der Neuen Institutionenökonomik. 2008.

Band 37 Jana Brandstätter: International divergierende demographische Entwicklungen und internationale Kapitalbewegungen. 2008.

Band 38 Annette Olbrisch: Die Zinsmarge und ihre Determinanten. Eine theoretische und empirische Analyse unter besonderer Berücksichtigung von Protektion im Bankensektor. 2009.

www.peterlang.de 